\title{
DE88 015056
}

\section{Small Angle Elastic Scattering of Protons off of Spinless Nuclei}

Alan Graham Ling*

\section{DISCLAIMER}

This report was prepared as an account of work sponsored by an agency of the United States Government. Neither the United States Government nor any agency thereof, nor any of their employees, makes any warranty, express or implied, or assumes any legal liability or respensibility for the accuracy, completeness, or usefulness of any information, apparatus, product, or process disclosed, or represents that its use would not infringe privately owned rights. Reference herein to any specific commercial product, process, or service by trade name, trademark, manufacturer, or otherwise does not necessarily constitute or imply its endorsement, recommendation, or favoring by the United States Government or any agency thereof. The views and opinions of authors expressed herein do not necessarily state or reflect those of the United States Government or any agency thereof.

"Guest Scientist at Los Alamos. Unizersity of California at Los Angeles. Los Angeles, CA 90024. 
TABLE OF CONTENTS

$$
\begin{array}{r}
\text { page } \\
v 1 \\
x \\
x 11 \\
x i v \\
x v
\end{array}
$$

List of Figures

List of Tables

Acknowledgements

Vita

Abstract

I. INTRODUCTION

II. THE THEORY OF SMALL ANGLE SCATTERING

A. THE COULOMB INTERACTION 3

B. COULOMB NUCLEAR INTERFERENCE 15

C. SPIN CONSIDERATIONS AND PARAMETERIZATION 34

D. DISPERSION RELATIONS $\quad 43$

III. THE EXPERIMENT

A. THE POLARIZED PROTON BEAM 61

B. LINE C AND THE HIGH RESOLUTION SPECTROMETER 67

C. DETECTORS $\quad 72$

D. ELECTRONICS AND DATA ACQUISITION 94

$\begin{array}{ll}\text { IV. DATA ANALYSIS } & 103\end{array}$

A. CALIBRATION OF DETECTOR SYSTEM 103

B. REPLAY OF THE DATA 109 
C. DETERMINATION OF CROSS SECTIONS

AND ANALYZING POHERS

V. RESULTS AND CONCLUSIONS

A. EXTRACTING THE REAL PART OF THE

NUCLEAR AMPLITUDE

B. PHYSICS OBTAINABLE FROM SMALL

ANGLE SCATTERING

C. PROBLEMS IN OBTAINING HIGH OUALITY

SMALL ANGLE DATA

REFERENCES

Appendix A: The HRS solid angle in the small angle region

Appendix B: Hultiple scattering

Appendix C: Statistical considerations of beam intensity monitoring

Appendix D: Tabulation of data 
page

Fig. 1 The contour of the integral of egn. (128) in 46 the complex $K$ plane.

Fig. 2 The direci s channel for proton scattering 48 on ${ }^{\circ} \mathrm{Ca}$.

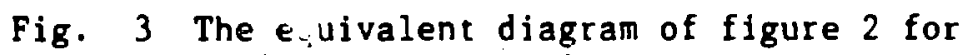
48 antiproton scattering.

Fig. 4 The exchange u channel for proton scattering on ${ }^{\circ} \mathrm{Ca}$.

Fig. 5 The equivalent diagram of figure 4 for antiproton scattering.

Fig. 6 Relativistic exchange processes for proton

Fig. 7 Relativistic exchange processes for antiproton scattering.

Fig. 8 Poles and branch cuts for p - ${ }^{10}$ Ca scattering. 54

Fig. 9 Ion sources for the $\mathrm{H}^{+}, \mathrm{H}^{-}$, and $\mathrm{P}^{-}$ beams at LAMPF.

Fig. 10 The experimental areas at LAMPF.

Fig. 11 Line $C$.

Fig. 12 The High Resolution Spectrometer (HRS).

Fig. 13 Focal plane detector arrangement (viewed from above) for the case of beam passing through the detectors.

Fig. 14 Side view of chamber showing each of the constituent planes $(a=c=9 \mathrm{~mm}, b=d=$ $e=9.5 \mathrm{~mm}$ ).

Fig. 15 Cross sectional view of an epoxied anode vire.

Fig. 16 Dotplot of X vs $Y$ anode wire positions in a chamber 79 in which the wires of one plane have been coated with epoxy in the center of the chamber.

Fig. 17 Histogram of wires in the $Y$ plane in the region without epoxy (top); $Y$ wires in the epoxied region (bot tom). 
Fig. 18 Delay line drift chamber used in this experiment

$(a=5.0 \mathrm{~cm}, b=15.0 \mathrm{~cm}, c=16.0 \mathrm{~cm}, d=5.0 \mathrm{~cm}$, $e=24.0 \mathrm{~cm}, f=16.0 \mathrm{~cm}, g=9.2 \mathrm{~cm}, h=5.72 \mathrm{~cm}$, $i=4.21 \mathrm{~cm})$.

Fig. 19 Location of odd and even cathode wires.

Fig. 20 ADC spectra of signals coming from cathode wires.

Fig. 21 Dot-plot of odd versus even ADC's.

Fig. 22 Dot-plot of OME versus position in chamber.

Fig. 23 Histogram of OME with events from all anode wires included (top), and with events from only one anode wire included (bot tom).

Fig. 24 Same as figure 23 except that only events in ADC channels greater than 250 are included.

Fig. 25 The method of offset planes to resolve

the left-right ambiguity.

Fig. 26 Event trigger logic.

Fig. 27 The electronics used to handle chamber signals.

Fig. 28 Trigger fan-out logic.

Fig. 29 Data acquisition system. Only one of several

Fig. 30 Wire and slit configuration for calibrating $\theta_{V}$. $z=79.22 \mathrm{~cm}, s=1.27 \mathrm{~cm}$.

Fig. 31 Vertical scattering angle at the target vs. $x$ at the focal plane.

Fig. 32 Wire and slit cunfiguration for calihrating $\theta_{\mathrm{H}}$. $z=79.22 \mathrm{~cm}, s=1.27 \mathrm{~cm}, y=0.5 \mathrm{~cm}, \theta_{\mathrm{s}}=9.2^{\circ}$.

Fig. 33 Dot-plot of TOF vs, pulse height.

Fig. 34 The effect of restricting the vertical scattering angle at the target as explained in the text.

Fig. 35 View of the focal plane 1lluminated with $\mathrm{p}-208 \mathrm{~Pb}$ quasi-elastic events.

Fig. 36 Position of elastic line on the focal plane with the HRS at $-0.83^{\circ}$. 
Fig. 37 Position of elastic line on the focal plane with the HRS at $2.5^{\circ}$.

Fig. 38 Location of scattering angle bins in chamber $1 Y$.

Fig. 39 Angular dependence of chamber efficiency for

Fig. 40 Angular dependence of chamber efflciency for $Y$ planes.

Fig. $41 X, Y$, and total chamber efficiencies.

Fig. 42 Scintillator configuration for the line C polarimeter in the up-down plane. LCPOUN * $51 \cdot 52 \cdot 53 \cdot 54, L C P O D N=55 \cdot 56 \cdot 57 \cdot 58$.

The left-right plane is similar.

Fig. 43 Dark current per beam gate of ERO2 for normal beam.

127

Fig. 44 Differential cross sections for protons on $12 \mathrm{C}$, ${ }^{\circ} \mathrm{Ca}$, and $208 \mathrm{~Pb}$ at $800 \mathrm{MeV}$.

Fig. 45 Analyzing powers for protons on ${ }^{12} \mathrm{C}$, ${ }^{10} \mathrm{Ca}$, and $20 * \mathrm{~Pb}$ at $800 \mathrm{MeV}$.

Fig. 46 Differential Cross section as a function of $\mathbf{q}^{2}$ for lead target.

Fig. 47 Differential Cross section as a function of $\mathrm{q}^{2}$ for calcium target.

Fig. 48 Differential Cross section as a function of $q^{2}$ for carbon target.

Fig. 49 Dependence of $\alpha$ at $q=0$ on beam energy.

Fig. 50 z-graph description of relativistic scattering.

Fig. 51 Graph describing non-relativistic scattering.

Fig. 52 Z-Graph showing virtual qā pair processes.

Fig. 53 Method of determining the solid angle of scattered particles.

Fig. 54 Position of scintillators used to count beam particles.

Fig. 55 Plot to determine the optimum setting of the table in the scattering chamber. 
Fig. 56 View of focal plane with(top) and without(bottom) events that scattered from the beam counting scintillators.

Fig. 57 Histogram of phi at the focal plane showing two peaks. The peak at left is from the beam counting scintillators.

Fig. 58 Differential cross sections for protons on ${ }^{\circ} \mathrm{Ca}$ and $20 . \mathrm{Pb}$ at $800 \mathrm{MeV}$.

Fig. 59 Ratio of differential cross sections measured at two 178 different beam intensities.

Fig. 60 Illustration showing that a unique normalization 181 constant and angular offset can be obtained from Coulomb scattering.

Fig. A Scattering of a particle in the lab frame. $\theta_{\mathrm{H}}$ is the angle described by $\underline{B A C}, Q_{V}$ by $\underline{C A D}$, $\rightarrow$ by $C B D$, and $\theta$ by $B A D$.

Fig. B Maximum out-of-plane scattering angle versus the polar angle $\theta$. The dashed line is an expanded $v$ iew of $\theta$ from $0^{\circ}$ to $9^{\circ}$.

Fig. C The solid angle acceptance of the HRS at $\theta_{S}=0^{\circ} . \quad 200$

Fig. D The solid angle acceptance of the HRS at $\theta_{S}=1.2^{\circ}$. 201

Fig. E The solid angle acceptance of the HRS at $\theta_{S}=2^{\circ}, \quad 201$

Fig. F The solid angle acceptance of the HRS at $\theta_{S}=4^{\circ}$. 202

Fig. G Solid angle dependence on $\theta$ for a $1 \mathrm{mr}$ bin size 208 at $\theta_{S}=0^{\circ}$.

Fig. H Solid angle dependence on $\theta$ for a $1 \mathrm{mr}$ bin size 208 at $\theta_{S}=1.2^{\circ}$.

Fig. I Solid angle dependence on $\theta$ for a $1 \mathrm{mr}$ bin size 209 at $\theta_{S}=2^{\circ}$.

Fig. J Solid angle dependence on $\theta$ for a $1 \mathrm{mr}$ bin size at $\theta_{S}=4^{\circ}$.

Fig. K Multiple scattering distribution for $800 \mathrm{MeV}$ protons 214 incident of a ${ }^{10} \mathrm{Ca}$ target $73.6 \mathrm{mg} / \mathrm{cm}^{2}$ thick.

Fig. L Multiple scattering distributions for the ${ }^{12} \mathrm{C},{ }^{10} \mathrm{Ca}$, and $20 . \mathrm{Pb}$ targets. 
page

Table 1 Exchange poles for p - $10 \mathrm{Ca}$ scattering. 51

Table 2 Two particle intermediate states in $\mathrm{p}-{ }^{\circ} \mathrm{Ca}$ scat tering.

Table 3 LAMPF Beam Parameters. 65

Table 4 HRS Specifications.

Table 5 Coefficients for determining $\theta_{V}$. 106

Table 6 Coefficients for determining $\theta_{\mathrm{H}}$. 108

Table 7 Normalization constants from Texas 130 p - $20 . \mathrm{Pb}$ data.

Table 8 Variants used to fit the data to Eqn.(169). 145

Table 9 Results of fits of lead data to variants 147 of table 8 .

Table 10 Results of fits of calcium data to variants 148 of table 8 .

Table 11 Results of its of carbon data to variants 149 of table 8 .

Table 12 Fermi Covariants used in IA1. 162

Table A Range of for $\theta_{S}=0^{\circ}$. 205

Table B Range of for $\theta_{S}=1.2^{\circ}$. 205

Table $c$ Range of for $\theta_{S}=2^{0}$. 206

Table $D$ Range of for $\theta_{S}=4^{\circ}$. 206

Table E Relative differential cross sections for 221

Table F Relative differential cross sections for 222 $10 \mathrm{Ca}(\mathrm{p}, \mathrm{p})$ at $800 \mathrm{MeV}$.

Table G Relative differential cross sections for 223 ${ }^{12} \mathrm{C}(\mathrm{p}, \mathrm{p})$ at $800 \mathrm{MeV}$.

Table $H$ Absolute differential cross sections for 224 $208 \mathrm{~Pb}(\mathrm{p}, \mathrm{p})$ at $800 \mathrm{MeV}$. 
Table I Absolute differential cross sections for ${ }^{10} \mathrm{Ca}(\mathrm{p}, \mathrm{p})$ at $800 \mathrm{MeV}$.

Table $J$ Absolute differential cross sections for ${ }^{2} \mathrm{C}(\mathrm{p}, \mathrm{p})$ at $800 \mathrm{MeV}$.

Table $\mathrm{K}$ Analyzing powers for $208 \mathrm{~Pb}(\mathrm{p}, \mathrm{p})$ at $800 \mathrm{MeV}$.

Table L Analyzing powers for ${ }^{10} \mathrm{Ca}(p, p)$ at $800 \mathrm{MeV}$. 229

Table $M$ Analyzing powers for ${ }^{12} \mathrm{C}(\mathrm{p}, \mathrm{p})$ at $800 \mathrm{MeV}$. 
ACKNOWLEDGENENTS

I an very grateful to my advisors George Igo and Chuck Whitten for their support and guidance on this project and for giving me the opportunities to learn experimental physics at LAMPF, Berkeley, and REK. For their patience and help in all aspects of this work, I am deeply indebted.

I would like to thank Dave Adams, Jiri Bystricky, and Mosh1 Moshi of UCLA and Doug Ciskowski and Marty Barlett of the University of Texas for their help in acquiring the data. To Jin Amann, Kevin Jones, and John McClelland of MP10, I owe special thanks for their technical assistance and valuable discussions. For help in acquiring the data referred to in this work as Exp916U, I would like to thank In addition to those mentioned above Vahe Ghazikhanian and Gerry Hof fmann.

I am very grateful to Val Armijo, Lee Atencio, Hal Mackay, and John Sandoval for their guidance and assistance in chamber development. I wish to thank Marek Bleszynski for the theoretical calculations presented in this work. And to all the DAC staff, I owe a sincere thankyou for making analysis of the data a smosth operation.

I would like to thank Charles Newsom for all that he has taught me during my early stay at LAMPF, and for his encouragement and inspiration. Thanks also to Mohini Rawool and Angel Vang for their friendship and concern. And to the administrative staffs of UCLA and LAMPF, In particular Bea Blonsky, Gloria Garcia, Ruth Gibson, Maxine 
Joppa, Penny Lucky, and Roberta Marinuzzi I say thanks for making my days as a graduate student a very pleasant time in my ilfe. The support of Associated Western Universities is also gratefully acknowledged. 


\title{
Small Angle Elastic Scattering of Protons off of Spinless Nuclei
}

by

\author{
Alan Graham Ling
}

ABSTRACT OF THE DISSERTATION

Elastic differential cross sections and analyzing powers for 800 MeV protons incident on ${ }^{12} \mathrm{C},{ }^{40} \mathrm{Ca}$, and ${ }^{208} \mathrm{~Pb}$ in the monentum transfer range $20 \mathrm{MeV} / \mathrm{c}<\mathrm{q}<130 \mathrm{MeV} / \mathrm{c}$ have been measured. The data was taken with the High Resolution Spectrometer (HRS) at the Los Alamos Meson Physics Facility. Special delay-line drift chambers with dead regions for the bean to pass through ther vere used to obtain the data. Through the interference of the Coulomb and nuclear contributions to the differential cross section in the small angle region, the ratio of the real to imaginary part of the forward nuclear amplitude $\alpha_{n}(0)=\operatorname{Ref}_{n}(0) / \operatorname{Imf}_{n}(0)$ is extracted. The importance of knowing this quantity at lover energies in order to study the differences between relativistic and non-relativistic scattering theories is discussed. 


\section{INTRODUCTION}

The interest in doing small angle elastic scattering experiments has been growing over the past several years. The reason for this is because data at small angles can be used to extract the absolute phase of the nuclear amplitude $\cot ^{-1} \alpha$ at $q=0$. This phase in combination with the other three independent measurable quantities $\mathrm{d} \sigma / d Q, A_{y}$, and $Q$ uniquely determine the scattering amplitude. Without knowledge of $\alpha$, the scattering amplitude is known only to within an arbitrary phase. The small angle region ts the only region in which the phase can be obtained, as it is here that the interference between the Coulomb and nuclear interactions can be exploited.

Zver since it was discovered that elastic p-Nucleus data, particularly the spin observables, could not be described vell by non-relativistic scattering theories, the search has been on for a theory that would describe this data. Various relativistic theories have met with some success and progress in the Dirac approach to nuclear scattering has advanced considerably. It is therefore advantageous to have data that is sensitive to relativistic scattering theorles. The as yet unmeasured phase of the forward nuclear amplitude for p-Nucleus elastic scattering is such a theory sensitive observable which can be used to test even the most popular relativistic scattering theory, the Dirac impulse approximation.

This work is a report on experimental progress tovard obtaining the nuclear phase. There are severe technical difficulties involved in obtaining small angle data for the purpose of extracting the 
phase. The presence of the beam near the angular region of interest is the primary obstacle to be dealt with. We describe a method of dealing with the problem of the beam passing through a system of detectors used to obtain the data presented in this work. The method consisted of making that part of the detector system through which the beam passed insensitive to the beam so that the small angle data nearby could be readily acquired. Months of testing and development were spent before a suitable technique was brought to the point where it would perform in the desired fashion. This work is therefore a description of the method and application of this technique to obtain the small angle data presented in this work.

In chapter II we discuss the theory behind small angle scattering. In particular, the interference between the Coulomb and nuclear interactions is shown to provide the mechanism through which the nuclear phase may be extracted. Utilization of a suitable parameterization of the nuclear amplitude in order to extract the phase is discussed. The dispersion relation approach for describing the real part of the nuclear amplitude is reviewed. Chapter III deals with the experimental arrangement of LAKPF experiment $916^{1}$. The method of overcoming the above mentloned technical difficulty is elaborately explained. In chapter IV, the analysis of the data is discussed. Finally, in chapter $V$ the real part of the forward nuclear amplitude is extracted from the data. The relevance of small angle data to discern between various scattering theories is discussed and methods to decrease the experimental uncertainty in the differential cross section are noted. 
II. THE THEORY OF SMALL ANGLE SCATTERING

In this section, the theoretical background governing small angle elastic scattering is discussed along with answers to questions of what can be learned from an experiment of this type. Even though at small momentum transfers information concerning the interior of a nucleus cannot be obtained, small angle elastic scattering is extremely interesting. It will be seen that because of the interference between the coulomb interaction and the strong interaction, the phase of the nuclear amplitude can be measured. In addition, one can predict the antinucleon cross sections.

\section{II-A. THE COULOMB INTERACTION}

The Coulomb interaction, al though well known, is not as simple to use in scattering theory as one might at first think. In fact no truly useful form for the pure Coulomb scattering amplitude exists. Limitations and approximations have to be applied before the theory can be put into a useful form. The main problem with the Coulomb interaction is its infinite range. No matter how far removed from the region of interaction one looks, the Coulomb interaction cannot be neglected. Consequently plane waves and spherical outgoing vaves do not really exist. The asymptotic wave function ${ }^{2}$

$$
\psi(r)=\psi_{\text {inc }}(r)+\psi_{\text {scat }}(r)=e^{i k z}+f(\theta) \frac{e^{i k r}}{r}
$$


which is at the heart of potential scattering theory cannot be used because the coulomb potential does not fall off faster than $1 / \mathrm{r}$. Thus either an entirely different approach must be taken, or approximations must be made.

To see the difficulty involved, we start with the Schrödinger equation for two particles of mass $m_{1}, m_{2}$ and charge $z_{1}, z_{2}$

$$
\hat{H} \psi(\vec{r})=E \psi(\vec{r})
$$

with

$$
\hat{\mathrm{H}}=\hat{\mathrm{H}}_{\mathrm{rel}}+\hat{\mathrm{H}}_{\mathrm{cm}}
$$

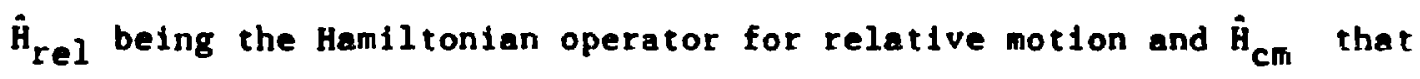
of the center of mass motion. The center of mass Hamiltonian is not relevant to our discussion and will thus be dropped. The Hamiltonian for relative motion between the two particles is

$$
\dot{\mathrm{H}}_{r e l}=\frac{\dot{\mathrm{p}}_{\mathrm{r}}^{2}}{2 \mu}+\frac{\hat{\mathrm{i}}^{2}}{2 \mu \mathrm{r}^{2}}+\mathrm{V}(\mathrm{r})
$$

with the Coulomb potential

$$
v(r)=\frac{z_{1} z_{2} e^{2}}{4 \pi \varepsilon_{0} r}
$$


Next, we use the fomiliar method of separation of variables to get a second order differential equation for the radial coordinate. Using

$$
\psi(\vec{r})=R_{1}(r) Y_{1}^{m}(\theta, \phi)
$$

and using the expression

$$
i^{2} Y_{1}^{m}(\theta, \phi)=1(1+1) K^{2} Y_{1}^{m}(\theta, \phi)
$$

we obtain from eqn.(2) with the help of eqn. (3)

$$
\left[\frac{\dot{p}_{r}^{2}}{2 \mu}+\frac{\hbar^{2} l(1+1)}{2 \mu r^{2}}+\frac{z_{1} z_{2} e^{2}}{4 \pi \varepsilon_{0} r}\right] R_{1}(r)=E R_{1}(r)
$$

Substituting $u_{1}(r)=r R_{1}(r)$ and using the relations

$$
\begin{gathered}
\dot{p}_{r}^{2} R(r)=-\frac{\hbar^{2}}{r} \frac{\partial}{\partial r}\left(\frac{\partial}{\partial r} u(r)\right)=-\frac{\hbar^{2}}{r} u^{\prime \prime}(r) \\
k^{2}=\frac{2 \mu E}{\hbar^{2}}
\end{gathered}
$$

eqn. (5) becomes

$$
u_{I}^{\mu}(r)+\left(k^{2}-\frac{2 \mu z_{1} z_{2} e^{2}}{h^{2} 4 \pi \varepsilon_{0} r}-\frac{1(l+1)}{r^{2}}\right) u_{1}(r)=0
$$


Let

$$
\beta=\frac{\mu z_{1} z_{2} e^{2}}{4 \pi \varepsilon_{o} k^{2} k}
$$

Then the differential equation is ${ }^{3}$

$$
u_{1}^{\prime \prime}(r)+\left(k^{2}-\frac{2 \beta k}{r}-\frac{1(1+1)}{r^{2}}\right) u_{1}(r)=0
$$

Ve now make three subst1tutions which w1ll transform eqn. (8) into a vell known form. First we let $p=k r$. Eqn.(8) becomes

$$
u_{1}^{\prime \prime}(p)+\left(1-\frac{2 \beta}{p}-\frac{1(1+1)}{p^{2}}\right) u_{1}(\theta)=0
$$

Then we let $z=-21 p$. Under this substitution we have

$$
-4 u_{1}^{\prime \prime}(z)+\left(1+\frac{413}{z}+\frac{41(1+1)}{z^{2}}\right) u_{1}(z)=0
$$

Finally, we make the transformation

$$
u_{1}(z)=e^{-z / 2}\left(\frac{12}{2}\right)^{1+1} y_{1}(z)
$$


After some algebra, the differential equation (8) in its final form is

$$
z y_{1}^{\prime}(z)+y_{1}(2(1+1)-z)-y_{1}(1+1+1 \beta)=0
$$

This is the vell known confluent hypergeometric differential equation with series solutions

$$
\begin{gathered}
y_{1}^{1}={ }_{1} F_{1}(a, c, z) \\
y_{1}^{2}=z^{1-c_{2} F_{1}(1+a-c, 2-c, z)}
\end{gathered}
$$

with $a=1+1+1 \beta$ and $c=2(1+1)$. These series solutions are known as the confluent hypergeometric functions or Kummer functions. When c is an integer $\geq 2$, as $i t$ is in this case, only the first solution exists . This is the regular solution. Therefore the solution to eqn. (12) is

$$
y_{1}(z)=C_{1}\left[{ }_{1} F_{1}(1+1+i \beta, 2(1+1), z)\right]
$$

or similarly the solution to eqn. (8) is

$$
\begin{aligned}
& u_{1}(r)=e^{i k r}(k r)^{1+1} C_{1}\left[{ }_{1} F_{1}(1+1+1 \beta, 2(1+1),-21 k r)\right] \\
& =e^{i k r}(k r)^{1+1} C_{1} \sum_{n=0}^{\infty} \frac{\Gamma(1+1+1 \beta+n) \Gamma(2(1+1))(-2 i k r)^{n}}{\Gamma(21+2+n) \Gamma(1+1+i \beta) n !}
\end{aligned}
$$

This is the exact solution to the problem of two particles 
Interacting by a Coulomb potential. It o'vicusly is not very useful. But let's proceed to obtain an asymptotic form for this solution analogous to the asymptotic form of eqn.(1). The asymptotic form for ${ }_{1} F_{1}(a, c, z)$ is

$$
\begin{aligned}
{ }_{1} F_{1}(a, c, z) & =\frac{\Gamma(c)}{\Gamma(a)} e^{z} z^{a-c} & & \text { Rez }>0 \\
& =\frac{\Gamma(c)}{\Gamma(c-a)}(-z)^{-a} & & \text { Rez<0 }
\end{aligned}
$$

Thus eqn. (13) asymptotically becomes

$$
\begin{gathered}
u_{r \rightarrow \infty}(r) \rightarrow(k r)^{1+1} c_{1} \frac{\Gamma(2(1+1))}{\Gamma(1+1+i \beta) \Gamma(1+1-i \beta)} \times \\
\left(\Gamma(1+1+1 \beta) e^{1 k r}(21 k r)^{-(1+1+1 \beta)}+\Gamma(1+1-1 \beta) e^{-1 k r}(-2 i k r)^{1 \beta-(1+1)}\right)
\end{gathered}
$$

After some manipulation we have

$$
u_{r \rightarrow \infty}(r) \rightarrow \frac{c_{1}(2 l+1) l e^{\pi / 2} \sin \left(k r-\beta \ln (2 k r)-\pi l / 2+\sigma_{1}\right)}{|\Gamma| 2^{1}}
$$

where we have set

$$
\Gamma=\Gamma(1+1+i \beta)
$$

and

$$
e^{i \sigma_{1}}=\left(\frac{\Gamma}{\Gamma^{\star}}\right)^{1 / 2}
$$


or

$$
\sigma_{1}=\arg \Gamma(1+1+1 \beta)
$$

We choose $c_{1}$ so that $u_{1}(r)=\sin \left(k r-\beta \ln (2 k r)-\pi l / 2+\sigma_{1}\right)$ :

$$
c_{1}=c_{1}(\beta)=\frac{|\Gamma| 2^{1}}{(21+1) \mid e^{\pi \beta / 2}}
$$

Defining

$$
F_{1}(\beta, k r)=e^{-i \sigma} 1 u_{1}(r)
$$

as the regular coulomb radial wavefunction, we can make the comparison of Coulomb scattering and free particle scatiering to see the nonusefulness of the exact solution. A partial wave analysis brings to light the difficulty at hand:

$$
\begin{aligned}
& \psi(\vec{r})=\frac{1}{k r} \sum_{1=0}^{\infty}(2 l+1) i^{1} u_{1}(r) P_{1}(\cos \theta) \\
& u_{1}=k r j_{1}(k r) \quad \text { free particle } \\
& u_{1}=e^{i \sigma_{1 F_{1}}(\beta, k r)} \quad \text { Coulomb }
\end{aligned}
$$

The free particle radial wavefunction reduces to the first term on the right-hand side of eqn.(1). The Coulomb radial savefunction reduces to

$$
\psi(\vec{r})=e^{i(k 2+\beta l n k(r-2)}+f_{c o u l}(\theta) \frac{e^{i(k r-\beta l n k r)}}{r}
$$


where

$$
f_{\operatorname{cosi}}(\theta)=-\frac{\beta e^{2 i \sigma_{0}-2 i \beta \ln s i n \theta / 2}}{2 k \sin ^{2} \theta / 2}
$$

Clearly, this asymptotic wavefunction does not have the form of a plane wave and a scattered wave. In addition, the differential cross section $d \sigma / d \theta=\left|f_{c o u l}(\theta)\right|^{2}$ is infinite in the forward direction. Thus the pure Coulomb interaction has a solution that cannot be applied in a meaningful way. An assumption has to be made to make any progress.

The problem is circumvented by assuming a shielded coulomb potential, which is a realistic assumption to make. It simply means that we take into account the electrons that shield the nucleus from the charged projectile. A few examples of commonly used screened Coulomb potentials are

$$
\begin{array}{cc}
v_{\text {coul }}(r)=\frac{z_{1} z_{2} e^{2}}{4 \pi \varepsilon_{0} r} e^{-r / a} & r \leq d \\
v_{\text {coul }}(r)=\frac{z_{1} z_{2} e^{2}}{4 \pi \varepsilon_{0} r} & r>d \\
0 &
\end{array}
$$

Using any Coulomb potential similar to these examples uhere the scattering potential decreases faster than $1 / \mathrm{r}$ simplifies the problem greatly. At large angular momenta (large impact parameters) the 
solution to the Schrödinger equation reduces to the free particle solution. Therefore an asymptotic wavefunction of the form of eqn. (1) can be used and we have a partial wave expansion of the usual form

$$
f(\theta)=\frac{1}{21 k} \sum_{1=0}^{\infty}(2 l+1)\left(e^{2 i \delta} 1-1\right) P_{1}(\cos \theta)
$$

Now let

$$
L=k d
$$

with a distance of the order of atomic dimensions, and ${ }^{3}$

$$
\begin{aligned}
& \delta_{1}=\quad \sigma_{1}-\beta \ln 2 \mathrm{kd} \quad 1 \leq \mathrm{L} \\
& \begin{array}{lll}
0 & 1>L
\end{array}
\end{aligned}
$$

That this is the correct form for the phase shift $\delta_{1}$ can be readily seen by comparing the asymptotic free particle and Coulomb radial wavefunctions at the boundary $r=d$ for the potential of eqn.(23):

$$
\begin{array}{ll}
\sin \left(k d-\pi l / 2+\delta_{1}\right) & \text { free } \\
\sin \left(k d-\pi l / 2+\sigma_{1}-\beta \ln 2 k d\right) & \text { Coulomb }
\end{array}
$$

with the free particle phase shift $\delta_{1}$ going to zero for $1 \gg \mathrm{kd}$. However, because of the sharp cutoff in the shielded Coulomb potential, we take $\delta_{1}$ to be zero at the boundary instead of decreasing slowly to zero. It must be noted that the shielded potential we have chosen does not give a phase shift with this 
behavior. But because the detectors are far away in comparison to $r$ - d, we are justified in setting $\delta_{l}=0$ for all $l>$ kd since this will not in the end affect the physical interpretation. Clearly this choice is made simply for ease of calculation. One could in fact envision a shielded potential that does give a phase shift with this behavior. The exact form depends on the shielding but the end result is the same.

The value of $L$ is very large. $d$ is of the order of $10^{-8} \mathrm{~cm}$ and for a proton of kinetic energy $800 \mathrm{MeV}, k=7 \times 10^{13} \mathrm{~cm}^{-1}$. Thus $\mathrm{L}$ $7 \times 10^{5}$ and because of this high value, only at extremely small angles does one have to worry about corrections to the Coulomb amplitude as we shall see.

Proceeding from eqn.(24), and setting $\lambda=\beta \ln (2 \mathrm{kd})$ we have

$$
\begin{aligned}
f(\theta) & =\frac{1}{21 k} \sum_{l=0}^{L}(2 l+1)\left(e^{2 i\left(\sigma_{1}-\lambda\right)}-1\right) P_{1}(\cos \theta) \\
= & \frac{e^{-2 i \lambda}}{2 i k} \sum_{l=0}^{L}(2 l+1)\left(e^{\left.2 i \sigma_{1}-1\right) P_{1}(\cos \theta)}\right. \\
& +\frac{e^{-2 i \lambda}-1}{2 i k} \sum_{l=0}^{L}(21+1) P_{1}(\cos \theta)
\end{aligned}
$$

since

$$
\frac{1}{2 i k} \sum_{l=0}^{\infty}(21+1)\left(e^{\left.2 i \sigma_{1}-1\right) p_{1}(\cos \theta)=f_{\operatorname{coul} l}(\theta)}\right.
$$

which is another form for eqn.(21), we have 


$$
f(\theta)=e^{-21 \lambda_{f o u l}}(\theta)+f_{>}+f_{<}
$$

where $\mathrm{e}^{6}$

$$
\begin{gathered}
f_{>}=-\frac{e^{-2 i \lambda}}{2 i k} \sum_{1=L+1}^{\infty}(21+1)\left(e^{\left.2 i \sigma_{1}-1\right) P_{1}(\cos \theta)}\right. \\
f_{<}=\frac{e^{-2 i \lambda}-1}{2 i k} \sum_{l=0}^{L}(2 l+1) P_{1}(\cos \theta)
\end{gathered}
$$

In most cases, the contributions from $f_{>}$and $f_{<}$are negligible. To see this, we replace the summation in each of these expressions with an integral. The first term becomes

$$
f_{>}=-\frac{e^{-2 i \lambda}}{1 k} \int_{L+1}^{\infty} l d l\left(e^{2 i \sigma} 1-1\right) P_{\lambda}(\cos \theta)
$$

Taking Stirling's approximation for the I function?

$$
\Gamma(1+1+1 \beta)=(1+1+i \beta)^{1+1 / 2+1 \beta_{e}-(1+1+1 \beta)}(2 \pi)^{1 / 2}
$$

we have

$$
e^{2 i \sigma_{1}}=\frac{T}{\Gamma^{*}}=(1+1)^{2 i \beta}
$$

And since $L \gg 1, P_{1}(\cos \theta) \simeq J_{0}(1 \theta)$ and thus

$$
f_{>}=-\frac{e^{-2 i \lambda}}{i k} \int_{L+1}^{\infty} I d I\left[(1+1)^{2 i \beta_{-}}-1\right] J_{0}(1 \theta)
$$


This integral has a significant value ${ }^{3}$ only for $\theta \leq 2 \beta / L$. Since $\beta$ is of the order of 1 and $L$ is of the order of $2 \times 10^{5}, f$, can be neglected at scattering angles greater than roughly $10^{-5}$ rad. To evaluate $f_{<}$we note that

$$
\sum_{l=0}^{L}(2 I+1) P_{1}(\cos \theta) \approx 2 \int_{0}^{L} 1 d I J_{0}(I \theta)=\frac{2 L}{\theta} J_{1}(L \theta)
$$

which is significant for $\theta \leq 1 / L$. Thus $f_{<}$can be neglected at scattering angles greater than $5 \times 10^{-4}$ rad. The physical interpretation of $f_{<}$is scattering of the incident waves of $f$ of the shielded charge, ie. diffraction caused by the sharp cutoff of the Coulomb potential at the spherical boundary. Eqn.(29) can thus be reduced to

$$
f(\theta)=e^{-2 i \lambda_{f o u l}}(\theta)
$$

with the stipulation that $\theta$ be greater than $10^{-4}$ rad which is easily satisfied in most experiments. The amplitude represented by eqn. (27) is finite at all angles whereas the amplitude for the unshielded potential is not. When $\theta$ is small enough that $f_{\text {, is significant and }}$ nust be included, $f_{>}$causes the total amplitude to be finite even at $\theta=0$ by removing the singularity there. One other observation is that the phase $e^{-2 i \lambda}$ is unmeasurable at present angular resolutions. But one could forsee measuring this phase by looking at the interference between $f_{c}$ and $f_{>}+f_{<}$at extremely small angles, taking data points between 0 and $10^{-5}$ rad 


$$
\frac{d \sigma}{d \ell}=\left|f_{c}\right|^{2}+f_{c}\left(f_{>}+f_{<}\right)^{\star} e^{-2 i \lambda}+f_{c}{ }^{\star}\left(f_{>}+f_{<}\right) e^{2 i \lambda}+\left|f_{\rangle}+f_{<}\right|^{2}
$$

However, the phase of the nuclear amplitude can be measured in a similar fashion and that is the subject of the next section.

\section{II-B. COULOMB NUCLEAR INTERFERENCE}

It is clear from the discussion in the previous section that in deaing with the physical situation of scattering with both the Coulomb and the nuclear interactions present one cannot simply write down

$$
f(\theta)=f_{\text {coul }}(\theta)+f_{\text {nuc }}(\theta)
$$

and proceed to measure quantities. In fact using eqn.(38) would result in measuring the interference between the Coulomb interaction and a residual nuclear interaction. What is desired is measurement of the real part of the pure nuclear amplitude, i.e. the amplitude resulting from the situation of scattering with the Coulomb interaction turned off. Eqn.(38) vould not yield this amplitude. Ve shall see that the presence of the Coulomb interaction rotates the nuclear amplitude in a manner analogous to rotation of isospin vectors in isospin space.

The starting point is the partial vave expansion eqn.(24) and what followed that discussion, except that now there are three 
regions to consider instead of two. Again we use the more realistic shielded Coulomb potential that abruptly goes to zero at the radius $d$ corresponding to the surrounding electron cloud. The nuclear potential, whose exact form is unknown, becomes considerably weaker than the Coulomb interaction at a radius $R$ somewhere between 0 and $d$ and can thus be neglected at distances much greater than $R$. Thus eqn. (26) can be written for this case as

$$
\begin{array}{ll}
\delta_{1}=\sigma_{1}+\delta_{1}^{n}-\beta \ln (2 k d) & 1 \leq L_{1} \\
\sigma_{1}-\beta \ln (2 k d) & L_{1}<1 \leq L_{2} \\
0 & L_{2}<1
\end{array}
$$

where $\delta_{1}^{n}$ is the nuclear phase shift, $L_{1}=k R, L_{2}=k d$. In the first region both the Coulomb and nuclear potentials are important while in the second region only the Coulomb interaction contributes. In the third region there is no interaction potential. $L_{2}$ is of the order of $10^{5}$ as before. In contrast $L_{1}$ is much smaller since $R$ is of the order of nuclear dimensions, ie. a fev $\mathrm{fm}$. For an 800 Hev proton scattering on ${ }^{12} \mathrm{C}, k=7.4 \mathrm{fm}^{-1}$ and $R=2-3 \mathrm{fm}$ so $L_{1}$ is of the order of 20 .

With the notation $\lambda=\beta \ln (2 \mathrm{kd})$, the scattering amplitude can be writen

$$
f(\theta)=\frac{1}{2 i k} \sum_{1=0}^{\infty}(21+1)\left(e^{2 i \delta} 1-1\right] P_{1}(\cos \theta)
$$




$$
\begin{aligned}
=\frac{1}{2 i k} & \sum_{1=0}^{L_{1}}(21+1)\left[e^{21\left(\sigma_{1}+\delta \delta_{1}^{n}-\lambda\right)}-1\right] P_{1}(\cos \theta) \\
& +\frac{1}{21 k} \sum_{1=L_{1}+1}^{L_{2}}(21+1)\left[e^{2 i\left(\sigma_{1}-\lambda\right)}-1\right] P_{1}(\cos \theta)
\end{aligned}
$$

Proceeding as before, eqn.(40) can be expressed in a more sultable form. The first term on the right side can be rewritten

$$
\begin{aligned}
& \frac{1}{2 i k} \sum_{1=0}^{L_{1}}(21+1)\left(e^{21\left(\sigma_{1}+\delta_{1}^{n}-\lambda\right)}-1\right] P_{1}(\cos \theta) \\
& =\frac{e^{-21 \lambda}}{2 i k} \sum_{l=0}^{L_{1}}(21+1)\left[e^{21\left(\sigma_{1}+\delta_{1}^{n}\right)}-1\right] P_{1}(\cos \theta) \\
& +\frac{\left(e^{-2 i \lambda}-1\right)}{2 i k} \sum_{l=0}^{L_{1}}(2 l+1) P_{1}(\cos \theta) \\
& =\frac{e^{-21 \lambda}}{21 k} \sum_{1=0}^{L_{1}}(21+1) \int e^{2 i \sigma_{1}}\left(e^{2 i \delta_{1-1}^{n}}\right) \int P_{1}(\cos \theta) \\
& +\frac{e^{-2 i \lambda}}{2 i k} \sum_{1=0}^{l_{1}}(21+1)\left(e^{\left.21 \sigma_{1}-1\right] P_{1}(\cos \theta)}\right. \\
& +\frac{\left(e^{-2 i \lambda}-1\right)}{2 i k} \sum_{l=0}^{L_{1}}(2 l+1) P_{1}(\cos \theta) \\
& =f_{n}^{r} e^{-2 i \lambda}+\frac{e^{-2 i \lambda}}{21 k} \sum_{1=0}^{L_{1}}(21+1)\left[e^{\left.2 i \sigma_{1}-1\right] P_{1}(\cos \theta)}\right. \\
& +\frac{\left(e^{-2 i \lambda}-1\right)}{2 i k} \sum_{1=0}^{L_{1}}(2 l+1) P_{1}(\cos \theta)
\end{aligned}
$$

vhere 


$$
f_{n}^{r}=\frac{1}{2 i k} \sum_{l=0}^{l_{1}}(21+1)\left(e^{21 \sigma_{1}}\left(e^{21 \delta_{l-1}^{n}}\right) j P_{1}(\cos \theta)\right.
$$

is the residual nuclear scattering amplitude. The second term on the right side of eqn.(40) can likewise be manipulated

$$
\begin{aligned}
& \frac{1}{2 i k} \sum_{1=L_{1}+1}^{L_{2}}(21+1)\left[e^{2 i\left(\sigma_{1}-\lambda\right)}-1\right] P_{1}(\cos \theta) \\
& =\frac{e^{-2 i \lambda}}{21 k} \sum_{1=L_{1}+1}^{L_{2}}(21+1)\left[e^{\left.2 i \sigma_{1}-1\right] P_{1}(\cos \theta)}\right. \\
& +\frac{\left(e^{-2 i \lambda}-1\right)}{21 k} \sum_{1=L_{1}+1}^{L_{2}}(21+1) P_{1}(\cos \theta)
\end{aligned}
$$

Combining the two rewritten forms of the two terms of eqn.(40), (eqns. (41) and (43)) we have

$$
\begin{aligned}
& f(\theta)= i_{n}^{r} e^{-21 \lambda}+\frac{e^{-2 i \lambda}}{2 i k} \sum_{l=0}^{L_{2}}(21+1)\left[e^{\left.2 i \sigma_{1}-1\right] P_{1}(\cos \theta)}\right. \\
&+\frac{\left(e^{-2 i \lambda}-1\right)}{21 k} \sum_{l=0}^{L_{2}}(21+1) P_{1}(\cos \theta) \\
&=f_{n}^{r} e^{-2 i \lambda}+\frac{e^{-2 i \lambda}}{2 i k} \sum_{l=0}^{\infty}(21+1)\left[e^{\left.2 i \sigma_{1}-1\right] P_{1}(\cos \theta)}\right. \\
& \quad-\frac{e^{-2 i \lambda}}{21 k} \sum_{1=L_{2}+1}^{\theta}(21+1)\left[e^{\left.2 i \sigma_{1}-1\right] P_{1}(\cos \theta)}\right. \\
& \quad+\frac{\left(e^{-2 i \lambda}-1\right)}{2 i k} \sum_{l=0}^{L_{2}}(21+1) P_{1}(\cos \theta)
\end{aligned}
$$


or

$$
f(\theta)=e^{-21 \lambda}\left(f_{c}+f_{n}^{r}\right)+f_{1}+f_{2}
$$

where

$$
f_{c}=\frac{1}{21 k} \sum_{l=0}^{\infty}(21+1)\left[e^{\left.21 \sigma_{1-1}\right] P_{1}(\cos \theta)}\right.
$$

is the Coulomb scattering amplitude, eqn.(28), and

$$
\begin{gathered}
f_{1}=-\frac{e^{-21 \lambda}}{21 k} \sum_{1=L_{2}+1}^{\infty}(21+1)\left[e^{21 \sigma} 1-1\right] P_{1}(\cos \theta) \\
f_{2}=\frac{\left(e^{-21 \lambda}-1\right)}{21 k} \sum_{1=0}^{L_{2}}(21+1) P_{1}(\cos \theta)
\end{gathered}
$$

Comparing eqn.(45) with eqn. (38), we see that there is a considerable difference bectreen the partial wave expansion and the naive assumption that the Coulomb and nuclear amplitudes are simply additive. However, $f_{1}$ and $f_{2}$ are recognized to be equal to $f_{>}$and $f_{<}$ (eqns.(30) and (31)) respectively. These vere shown to contribute significantly at scattering angles less than $-10^{-4} \mathrm{rad}$. Thus the scattering amplitude eqn. (45) reduces to

$$
f(\theta)=a^{\left.-2 i \lambda_{(} f_{c}+f_{n}^{r}\right)}
$$

provided that the scattering angle is greater than $\sim 10^{-4}$ rad. For 
this experiment, the angular range requirement for eqn.(49) is satisfied and ve can use it with the unobservable phase factor $\exp (-2 i \lambda)$ dropped. Eqn. (49) then becomes eqn. (38) with the exception that the nuclear amplitude measured is actually the iesidual nuclear amplitude eqn.(42), 1.e. the pure nuclear amplitude modified by the Coulomb phase factor $\exp \left(21 \sigma_{1}\right)$. That is desired is a measurement of the real part of the pure nuclear amplitude. Eqn.(42) must then be rewritten in terms of the pure nuclear amplitude. Unfortunately this cannot be done without making an approximation. In fact an attempt to factor out $\sigma_{1}$ from the summation leads to a pure nuclear amplitude $f_{n}$ of the form

$$
\begin{aligned}
& f_{n}=f_{n}^{r} e^{-2 i \sigma_{0}}-2 i \sum_{l=1}^{\infty}(21+1) f_{1}^{n} P_{1}(\cos \theta) e^{2 i \sigma_{1} x} \\
& \left(\sigma_{1}-\sigma_{\theta}\right) e^{-1\left(\sigma_{1}-\sigma_{0}\right)} \prod_{k=1}^{\infty}\left(1-\frac{\left(\sigma_{1}-\sigma_{0}\right)^{2}}{k^{2} n^{2}}\right)
\end{aligned}
$$

where

$$
f_{1}^{n}=\frac{e^{21 \delta_{1}^{n}}-1}{2 i k}
$$

and

$$
f_{n}^{r}=\sum_{1=0}^{\infty}(21+1) e^{2 i \sigma_{1 f} f_{1}^{n} P_{1}(\cos \theta)}
$$

is the residual nuclear amplitude eqn.(42). If 1 t vere not for the second term of eqn.(50), we could simply replace $f_{n}^{r}$ in eqn. (49) with 
$f_{n} \exp \left(2 i \sigma_{0}\right)$ and proceed to measure the real part of $f_{n}$. The second term of eqn.(50) is, hovever, not to be neglected and we must either struggle through with it or make an approximation that will put the amplitude in a more workable form. The problem in trying to express the pure nuclear amplitude as a reasonable function of the residual amplitude is that the Coulomb phase factor $\exp \left(2 i \sigma_{1}\right)$ depends on 1 and cannot be removed from the summation. However, if we were to make the approximation that $\sigma_{1}$ is independent of 1 , then we would have a residual amplitude which is just the pure nuclear amplitude multiplied by some phase factor

$$
f_{n}^{r}=e^{i \phi} \sum_{l=0}^{\infty}(2 l+1) f_{1}^{n} P_{l}(\cos \theta)=e^{i \phi} f_{n}
$$

The problem would then reduce to calculating the phase factor $\phi$. We take this approach justifying the non 1 dependence of $\sigma_{1}$ by noting that small 1 values are the main contributing factors to the residual amplitude $f_{n}^{r}$ since the nuclear force is short range. And for small 1 values, $\sigma_{1}$ does not change appreciably. This can be seen from the fact that ${ }^{3}$

$$
\sigma_{1}=\sigma_{0}+\sum_{k=1}^{1} \tan ^{-1}\left(\frac{\beta}{k}\right)
$$

Since $\beta$ is of the order of 1 , the series converges rapidly and $\sigma_{1}$ is approximately independent of 1. Therefore eqn.(52) holds true and ve use for the average nuclear Coulomb phase shift $\sigma_{n}$ 


$$
f_{n}^{r}=e^{21 \sigma_{n} f_{n}}
$$

with

$$
f_{n}=\frac{1}{2 \pi k} \sum_{l=0}^{\infty}(21+1)\left(e^{2 i \delta_{1-1}^{n}} P_{1}(\cos \theta)\right.
$$

To find the average nuclear Coulomb phase shift on we start with the exact Coulomb phase shift for a point charge eqn.(4).

$$
\sigma_{1}=\arg \Gamma(1+1+1 \beta)
$$

from eqn. (33) this becomes in the limit of large 1

$$
\sigma_{1}=\beta \ln (1+1)=\beta \ln (k b)=\sigma(b)
$$

where $b$ is the impact parameter $b=1 / k$. For a point charge, the UKB approximation for the Coulomb phase shift, which is valid only for large 1 (or equivalently b), is written as ${ }^{\circ}$

$$
\sigma_{w}(b)=\lim _{r \rightarrow \infty}-\frac{\mu}{h^{2} k} \int_{b}^{r} \frac{v_{\text {coul }} r d r}{\left(r^{2}-b^{2}\right)^{1 / 2}}
$$

Substituting the polnt charge potential eqn.(4) into this expression gives

$$
\sigma_{w}(b)=\lim _{r \rightarrow \infty}-\beta(\ln (2 k r)-\ln (k b))
$$

Unfortunately, the limit as $r+\infty$ of eqn.(58) does not exist. The 
reason for this is due to the fact that the Coulomb potential for a point charge does not decrease faster than $1 / r$. However, instead of taking the limit as $r+\infty$, we can take the limit to some arbitrary point $R$ to make the integration of eqn.(57) fintte and assign the remaining contribution to a constant $c$.

$$
\begin{aligned}
\sigma_{v}(b) & =-\beta \int_{b}^{R} \frac{d r}{\left(r^{2}-b^{2}\right)^{1 / 2}}+c \\
& =-b \ln (2 R / b)+c
\end{aligned}
$$

Since $\sigma_{w}(b)$ must equal the asymptotic form for the exact Coulomb phase shift eqn.(56), we can equate eqns.(56) and (59) to find C.

$$
\beta \ln (k b)=-\beta \ln (2 R / b)+C
$$

or

$$
C=\beta \ln (2 k R)
$$

Thus the asymptotic phase shift for a point charge is

$$
\begin{gathered}
\sigma(b)=\sigma_{w}(b) \\
=-\frac{\mu}{\hbar^{2} k} \int_{b}^{R} \frac{v_{c o u l} r d r}{\left(r^{2}-b^{2}\right)^{1 / 2}}+\beta \ln (2 k R)
\end{gathered}
$$

with $V_{\text {coul }}$ the point charge potential.

Eqn.(60) holds only for large $b$ as that is where the WKB approximation is valid. However, if we use a Coulomb potential that 
is smooth and finite everywhere, the WKB approximation will be valid at all b so that the asymptotic form for the phase shift will be good for all phase shifts. A suitable Coulomb potential which has the desired properties results from using a gaus: an charge distribution for a nucleus.

$$
p(r)=\frac{1}{\varepsilon^{3} \pi^{3 / 2}} e^{-(r / \varepsilon)^{2}}
$$

with $\varepsilon$ is a constant chosen to give an appropriate charge rms radius, e.g. $\varepsilon=2.40 \times 10^{-13} \mathrm{~cm}$ for ${ }^{12} \mathrm{c}$ determined from elastic electron scattering ${ }^{9}$. This charge distribution is the solution of $\nabla \cdot \vec{E}=p / \varepsilon_{0}$ with

$$
E=\frac{1}{4 \pi \varepsilon_{0}}\left[\frac{1}{r^{2}} \operatorname{erf}(r / \varepsilon)-\frac{2}{\varepsilon r \pi^{1 / 2}} \exp \left(-(r / \varepsilon)^{2}\right)\right] \dot{r}
$$

with erf the error function

$$
\operatorname{erf}(x)=\frac{2}{\pi^{1 / 2}} \int_{0}^{x} \exp \left(-t^{2}\right) d t
$$

Since $-\sigma_{\text {coul }}=E$, the Coulomb potential will be

$$
v_{\text {coul }}=\frac{z_{1} z_{2} e^{2}}{4 \pi \varepsilon_{0} r} \operatorname{erf}(r / \varepsilon)
$$

Eqn. $(60)$ is then 


$$
\sigma(b)=-\beta \int_{b}^{R} \frac{e r f(r / \varepsilon) d r}{\left(r^{2}-b^{2}\right)^{1 / 2}}+\beta \ln (2 k R)
$$

Using

$$
-\beta \int_{b}^{R} \frac{d r}{\left(r^{2}-b^{2}\right)^{1 / 2}}=-\beta \ln (2 R / b)
$$

eqn.(62) can be written in a more useful form

$$
\sigma(b)=\beta\left(\int_{\frac{b}{\varepsilon}}^{\infty} \frac{(1-\operatorname{er} f(x)) d x}{\left(x^{2}-\frac{b^{2}}{\varepsilon^{2}}\right)^{1 / 2}}+\ln (k b)\right)
$$

having taken the limit $R \rightarrow-$ and setting $x=r / \varepsilon$. From this form for the Coulomb phase shift we can find the average Coulomb phase shift of the nuclear scattering amplitude. It is found by the standard way of calculating averaged quantities, namely

$$
\sigma_{n}=\frac{\int_{0}^{\infty} f(b) \sigma(b) b d b}{\int_{0}^{\infty} f(b) b d b}
$$

wth $f(b)$ a suitably chosen velghting function. For the purpose at hand, reasonable function is

$$
f(b)=C e^{-b^{2} / \varepsilon^{2}}
$$


This cholce reflects the gaussian charge distribution of eqn.(61). Substituting eqns.(66) and (64) into eqn.(65) and performing the integration, we find

$$
\sigma_{n}=\beta \ln (k \varepsilon)
$$

Going back to eqn.(54), we can see what effect the coulomb interaction has on the nuclear amplitude. The coulomb interaction rotates the pure nuclear amplitude through an angle $2 \sigma_{n}$ to give the experimentally observed residual nuclear amplitude

$$
\left(\begin{array}{l}
\operatorname{Im} f_{n}^{r} \\
\operatorname{Re} f_{n}^{r}
\end{array}\right)=\left(\begin{array}{cc}
\cos 2 \sigma_{n} & \sin 2 \sigma_{n} \\
-\sin 2 \sigma_{n} & \cos 2 \sigma_{n}
\end{array}\right)\left(\begin{array}{l}
\operatorname{Im} f_{n} \\
\operatorname{Ref}
\end{array}\right)
$$

This can be viewed in much the same way as the Idea that the coulomb interaction causes creation of an isospin space in which particle vectors can be rotated between each other. Without this coulomb interaction, there would be no isospin space and particle families vould become degenerate. Similarly, with no electromagnetic forces, $\sigma_{n}$ would vanish and the vectors $f_{n}$ and $f_{n}^{r}$ would be degenerate, with no rotations in "amplitude space".

Collecting eqns.(21), (49), and (54) we have for the total scattering amplitude

$$
f(\theta)=f_{c o u l}+e^{2 i \sigma_{n} f_{n u c}}=-\frac{B e^{2 i \sigma_{c}}}{2 k \sin ^{2} \theta / 2}+f_{n} e^{21 \sigma_{n}}
$$


with $\sigma_{c}=\sigma_{0}-\beta \ln (\sin \theta / 2)$. Since the differential cross section does not depend on the overall phase of $f(\theta)$, Eqn. (69) can be written as

$$
f(\theta)=-\frac{\beta e^{2 i \phi}}{2 k \sin ^{2} \theta / 2}+f_{n}
$$

wi th

$$
\phi=\sigma_{c}-\sigma_{n}=\sigma_{0}-\beta \ln (\sin \theta / 2)-\beta \ln k \varepsilon
$$

Since oo $\approx-\beta \gamma$, with $\gamma=.577=$ Euler's constani, becomes

$$
\phi=\beta \ln (1.123 / \theta k \varepsilon)
$$

where ve have used the approximation for small angies $\sin \theta / 2-\theta / 2$. From eqn. (70) we can calculate the real part of $f_{n}$ by measuring the differential cross section:

$$
\begin{gathered}
\frac{d \sigma}{d Q}=|f(\theta)|^{2}= \\
\frac{\beta^{2}}{4 k^{2} \sin ^{4} \theta / 2}-\frac{f_{n} \beta e^{-2 i \phi}}{2 k \sin ^{2} \theta / 2}-\frac{f_{n}^{\star} \beta e^{2 i \phi}}{2 k \sin ^{2} \theta / 2}+\left|f_{n}\right|^{2}
\end{gathered}
$$

The first term is pure Coulomb scattering and the last term is pure nuclear scattering. The second and third terms arise from the interference of the Coulomb and nuclear forces and provide the means of measuring the real part of the nuclear amplitude. Eqn.(73) can be 
expressed in terms of the real part, or more conveniently, the ratio of the real to imaginary part of the nuclear amplitude

$$
\begin{gathered}
\frac{d \theta}{d \phi}(\theta)=\frac{\beta^{2}}{4 k^{2} \sin ^{4} \theta / 2}-\frac{\beta}{k \sin ^{2} \theta / 2} \operatorname{Imf}(\alpha \cos 2 \phi+\sin 2 \phi) \\
+\left(\operatorname{Imf}_{n}\right)^{2}\left(\alpha^{2}+1\right)
\end{gathered}
$$

where

$$
\alpha=\frac{\operatorname{Ref}_{n}}{\operatorname{Imf}_{n}}
$$

In order to use eqn. (74) to measure $\alpha, \operatorname{Imf}_{n}$ must be known. Imf $f_{n}$ can be calculated from the optical theorem which is valid only at $0^{\circ}$. Therefore $\alpha$ can only be measured at $0^{\circ}$. But as was discussed before, eqn.(74) is only valid at angles greater than $-10^{-4}$ rad. To get around this $\mathrm{flx}$, we simply parameterize the nuclear amplitude and fit eqn. (74) to the measured values of the differential cross section to obtain the parameters and the amplitude at zero degrees. This will be developed in the next section.

Eqn.(74) is the general expression that describes elastic scattering of charged particles that interact strongly. However, it has been pointed out ${ }^{20}$ that for scattering off of large objects such as heavy nuclei, a correction to eqn.(74) may be necessary. To see this, we start with a Coulomb potential of the form 


$$
v_{\text {coul }}(r)=\frac{z_{1} z_{2} e^{2} e^{-n\left(r^{2}+\varepsilon^{2}\right)^{1 / 2}}}{4 \pi \varepsilon_{0}\left(r^{2}+\varepsilon^{2}\right)^{1 / 2}}
$$

where $n$ is a constant that will be seen to be insignificant in our considerations and $\varepsilon$ is a constant to make the potential less singular than $1 / r$ at the origin. Putting this into eqn.(57) and performing the integration yields for the Coulomb phase shift

$$
\sigma_{c}(b)=-B K_{0}\left(n\left(b^{2}+\varepsilon^{2}\right)^{1 / 2}\right)
$$

with $K_{0}$ the modified Bessel function. Now the integral representation of the Coulomb amplitude, eqn.(28) is

$$
E_{c}=\frac{k}{i} \int_{0}^{\infty} b d b J_{0}(b q)\left(e^{2 i \sigma_{c}(b)}-1\right)
$$

with $q=2 k \sin \theta / 2$. Substituting eqn. (77) Into eqn.(78) and using $\sigma_{0}$ - - Br gives for the Coulomb amplitude after some manipulation and for small $q$

$$
f_{c}=-\frac{\beta}{2 k \sin ^{2} \theta / 2} e^{2 i \sigma_{c}}
$$

with

$$
\sigma_{c}=\beta(\ln \eta-\ln (2 k \sin \theta / 2))
$$

This is the same as eqn.(21) except the phase is a little different. 
Bowever, what is important is the difference in phase eqn.(71) $\phi=\sigma_{c}$ - $\sigma_{n}$ which does not change in this consideration.

Instead of using the average Coulomb phase shift in the residual nuclear amplitude, we calculate it without making the approximation that it is independent of 1 . However we stick with the notion that only small values of 1 (or b) contribute significantly to the phase shift. Therefore eqn. (77) in the limit of small b reduces to

$$
\sigma_{c}(b)=\beta \ln \left(\frac{1}{2} n\left(b^{2}+\varepsilon^{2}\right)^{1 / 2}\right)+\beta r
$$

Taking the integral representation of the residual amplitude eqn.(42) and using eqn.(81) for $\sigma_{1}$ gives

$$
f_{n}^{r}=\frac{k}{i} \int_{0}^{\infty} b d b J_{0}(b q)\left(e^{2 i \delta^{n}(b)}-1\right) e^{i \beta \ln \left(1+b^{2} / \varepsilon^{2}\right)} e^{21 \beta(\ln (n \varepsilon / 2)+r)}
$$

Next we expand $\ln \left(1+b^{2} / \varepsilon^{2}\right)$ and use an optical model form for the nuclear phase shift

$$
e^{2 i \delta^{n}(b)}-1=-A e^{-2 b^{2} / R^{2}}
$$

which makes the residual amplitude

$$
f_{n}^{r}=k i A e^{2 i \beta(\ln (n \varepsilon / 2)+r)} \int_{0}^{\infty} b d b J_{0}(b q) e^{i \beta b^{2} / \varepsilon^{2}-2 b^{2} / R^{2}}
$$

After performing the integration we have 


$$
f_{n}^{r}=\frac{k i A}{\left(\frac{2}{R^{2}}-\frac{1 \beta}{\varepsilon^{2}}\right)} e^{2 i \beta(\ln (n \varepsilon / 2)+\gamma)} \exp \left[-\frac{1}{4} q^{2}\left(\frac{2}{R^{2}}-\frac{1 \beta}{\varepsilon^{2}}\right)^{-1}\right]
$$

We wish to express this in terms of the pure nuclear amplitude. With the nuclear phase shift given by eqn.(83) the pure nuclear amplitude obtained from an integral representation of eqn. (55) is

$$
f_{n}=\frac{1}{4} 1 k A R^{2} e^{-R^{2} q^{2} / B}
$$

Combining eqns. (85) and (86) we have, neglecting terms in $\beta^{2}$ and $q^{2} / \beta$

$$
f_{n}^{r}=\frac{E_{n} e^{2 i \sigma_{n}}}{1-\frac{i R^{2} \beta}{2 \varepsilon^{2}}}
$$

with $\sigma_{n}=B(\ln (n \varepsilon / 2)+r)$. Taking the total amplitude as the sum of eqns. (79) and (87) gives

$$
f(\theta)=-\frac{\beta}{2 k \sin ^{2} \theta / 2} e^{2 i \phi}+f_{n}\left(1-\frac{i R^{2} \beta}{2 \varepsilon^{2}}\right)^{-1}
$$

with $=\sigma_{c}-\sigma_{n}=\beta i n(1.123 / \theta k \varepsilon)$ the same as before. Ve compare this formula with eqn.(70) which was derived under the assumption that $\sigma_{1}$ was independent of 1 . Since $R$, the optical model radius, is of the order of $\varepsilon$, the correction factor in eqn.(88) is governed by 
the size of $\beta$. For Pp scattering $\beta$ is small, the correction factor can be neglected, and eqn.(88) reduces to eqn.(70). But since $B$ increases for p-nucleus scattering, the correction factor can become significant especially for heavy nuclei. The differential cross section calculated from eqn.(88) is then

$$
\begin{aligned}
\frac{d \sigma}{d \boldsymbol{Q}}(\theta)= & \frac{\beta^{2}}{4 \mathrm{k}^{2} \sin ^{4} \theta / 2}-\frac{\beta \operatorname{Imf} \mathrm{n}}{\rho \mathrm{ks} \sin ^{2} \theta / 2}\left[\alpha\left(\cos 2 \phi+\frac{\mathrm{R}^{2} \beta}{2 \varepsilon^{2}} \sin 2 \phi\right)\right. \\
& \left.+\left(\sin 2 \phi-\frac{\mathrm{R}^{2} \beta}{2 \varepsilon^{2}} \cos 2 \phi\right)\right]+\frac{\left(\operatorname{Im} \varepsilon_{\pi}^{\varepsilon}\right)^{2}}{\rho}\left(\alpha^{2}+1\right)
\end{aligned}
$$

where

$$
p=1+\frac{R^{4} \beta^{2}}{4 \varepsilon^{4}}
$$

Eqn.(89) is the correct formula to use to fit data for p-nucleus scattering when the $A$ of the nucleus is large enough that $B$ is sizeable. For particle scattering and for scattering from small nuclei, eqn. (74) suffices.

Fitting eqn.(89) to the measured differential cross section allows calculation of $\alpha$, or the real part of the nuclear amplitude. Knowing the real part enables us to predict the antinucleon cross section, in this case scattering of antiprotons of $f$ the same target nucleus. To see how this can be done, we write eqn. (89) for the case of antiparticle scattering. This is easily derived from the 
cross section for particle scattering by making the following transformations:

$$
\begin{aligned}
& \beta \rightarrow-\beta \\
& \phi \rightarrow-\phi \\
& f_{n} \rightarrow f_{n}
\end{aligned}
$$

Then the cross section for antiproton scattering is just

$$
\begin{aligned}
\frac{d \bar{\sigma}}{d \bar{Q}}(\theta) & =\frac{\beta^{2}}{4 k^{2} \sin ^{4} \theta / 2}+\frac{\beta \operatorname{Imf}}{\rho k \sin ^{2} \theta / 2}\left[\alpha\left(\cos 2 \phi+\frac{R^{2} \beta}{2 \varepsilon^{2}} \sin 2 \phi\right)\right. \\
& \left.+\left(-\sin 2 \phi+\frac{R^{2} \beta}{2 \varepsilon^{2}} \cos 2 \phi\right)\right]+\frac{(\operatorname{Imf})^{2}}{\rho}\left(\alpha^{2}+1\right)
\end{aligned}
$$

Combining eqns.(89) and (91), we have the following relation between particle and antiparticle scattering:

$$
\frac{d \vec{\theta}}{d \bar{Q}}=\frac{d \sigma}{d \bar{Q}}+\frac{2 B R e f}{\rho k \sin ^{2} \theta / 2}\left(\cos 2 \phi+\frac{R^{2} \beta}{2 \varepsilon^{2}} \sin 2 \phi\right)
$$

Thus we see that by measuring $d \sigma / d Q$ for proton scattering and fitting it to eqn.(89) to find Ref $n$, ve can calculate the antiproton differential scattering cross section $d \bar{\sigma} / d Q$. Incidentally, ve note that if we take both sides of eqn.(92) and integrate over the solid angle and take the limit $k \rightarrow \infty$, we obtain the familiar Poneranchuk theorem: 


$$
\underset{k \rightarrow \infty}{\bar{\sigma}}=\sigma
$$

In high energy particle scattering, where most of the experimental data on $\operatorname{Ref}_{n}$ exists, one usually measures both $\mathrm{d} \sigma / \mathrm{d} Q$ and $d \bar{\sigma} / d \Omega$ and then uses eqn.(92), with $p=1$ and the $\sin 2 \phi$ term dropped for particle-particle scattering, to calculate Ref ${ }_{n}$. This is easily done in colliding beam machines where, for example, $\pi^{+} p$ and $\pi^{-} p$ are equally easy to set up. In this experiment, there is no available antiproton beam which could be used as a check of eqn.(92). Furthermore, the data on $\bar{p}$-nucleus differential cross sections is extremely lacking. Thus the use of eqn.(92) to predict do/dQ should stimulate interest in the physics community to measure $\bar{p}$-nucleus cross sections.

\section{II-C. SPIN CONSIDERATIONS AND PARAMETERIZATION}

In the preceding section, we established the framework necessary to obtain the real part of the forward nuclear amplitude from measuring the elastic differential cross section. Eqn.(89) should be recognized as the key formula to be used in this calculation. However, this expression is for scattering of spinless particles only, and needs to be developed further if it is desired to get $\operatorname{Im} f_{n}$ for elastic scattering of particles with non-zero spin. In addition, it is necessary to parameterize the nuclear amplitude since the elastic differential cross section is a function of $\operatorname{Im} f_{n}(q)$ and not $\operatorname{Imf}_{n}(q=0)$. Thus the differential cross section will be a function of 
scattering angle (or momentum transfer) and a set of parameters to be determined.

We first consider the general case of elastic scattering of 2 spin $1 / 2$ particles and then apply the results to our particular case of protons scattering off of spinless nuclei. The obvious place to start the discussion is from the elastic scattering matrix 11 for 2 spin $1 / 2$ particles:

$$
\begin{gathered}
M_{n}=\alpha_{n}+i \gamma_{n}\left(\vec{\sigma}_{1}+\vec{\sigma}_{2}\right) \cdot \dot{n}+\beta_{n}\left(\vec{\sigma}_{1} \cdot \dot{n}\right)\left(\vec{\sigma}_{2} \cdot \dot{n}\right)+ \\
\delta_{n}\left(\vec{\sigma}_{1} \cdot \dot{m}\right)\left(\vec{\sigma}_{2} \cdot \dot{m}\right)+\varepsilon_{n}\left(\vec{\sigma}_{1} \cdot \dot{i}\right)\left(\vec{\sigma}_{2} \cdot \dot{i}\right)+1 \eta_{n}\left(\vec{\sigma}_{1} \cdot \dot{n}-\vec{\sigma}_{2} \cdot \dot{n}\right)
\end{gathered}
$$

and

$$
\begin{gathered}
M_{c}=\alpha_{c}+i \gamma_{c}\left(\vec{\sigma}_{1}+\vec{\sigma}_{2}\right) \cdot \dot{n}+\beta_{c}\left(\vec{\sigma}_{1} \cdot \dot{n}\right)\left(\vec{\sigma}_{2} \cdot \dot{n}\right)+ \\
\delta_{c}\left(\vec{\sigma}_{1} \cdot \dot{m}\right)\left(\vec{\sigma}_{2} \cdot \dot{m}\right)+\varepsilon_{c}\left(\vec{\sigma}_{1} \cdot \dot{j}\right)\left(\vec{\sigma}_{2} \cdot \dot{j}\right)+i \eta_{c}\left(\vec{\sigma}_{1} \cdot \dot{n}-\vec{\sigma}_{2} \cdot \dot{n}\right)
\end{gathered}
$$

Here we have scattering matrices for nuclear $\left(M_{n}\right)$ and Coulomb $\left(M_{c}\right)$ scattering so that the total scattering matrix is fust

$$
M_{\text {tot }}=M_{n}+M_{c}
$$

In these formulae, $\vec{\sigma}_{1}$ and $\vec{\sigma}_{2}$ are the Paull spin operators for the incident and target particles respectively and $\alpha_{n}, \alpha_{c}, r_{n}, r_{c}, \beta_{n}$, $\beta_{c}, \delta_{n}, \delta_{c}, \varepsilon_{n}, \varepsilon_{c}, \eta_{n}, \eta_{c}$ are the complex nuclear and Coulomb scattering amplitudes that are functions of energy and momentum transfer. The $\alpha^{\prime} s$ are the spin-independent amplitudes, the $\gamma^{\prime} s$ and $n^{\prime} s$ are the spin orbit amplitudes, and the $\beta^{\prime} s, \delta^{\prime} s$, and $\varepsilon^{\prime} s$ are the 
double spin-flip amplitudes. The unit vectors $i, \dot{m}, \dot{n}$ compose a right-handed Cartesian coordinate syster made from $\vec{k}_{1}$ and $\vec{R}_{f}$, the momentum vectors of the incident and scattered particles in the center of mass respectively.

$$
\begin{aligned}
& i=\frac{\vec{k}_{i}+\vec{k}_{f}}{\left|\vec{k}_{i}+\vec{k}_{f}\right|} \\
& \dot{m}=\frac{\vec{k}_{f}-\vec{k}_{i}}{\left|\vec{k}_{f}-\vec{k}_{i}\right|} \\
& \dot{n}=\frac{\vec{k}_{i} \times \vec{k}_{f}}{\left|\vec{k}_{i} \times \vec{k}_{f}\right|}
\end{aligned}
$$

For the simple case of an unpolarized beam incident on an unpolarized target, the differential cross section is

$$
\left(\frac{d \sigma}{d Q}\right)_{U N}=\left(\frac{d \sigma}{d Q}\right)_{N}+\left(\frac{d \sigma}{d Q}\right)_{C}+\left(\frac{d \sigma}{d Q}\right)_{I}
$$

with the pure nuclear contribution

$$
\left(\frac{d \sigma}{d Q}\right)_{N}=\left|\alpha_{n}\right|^{2}+\left|\gamma_{n}\right|^{2}+\left|\beta_{n}\right|^{2}+\left|\delta_{n}\right|^{2}+\left|\varepsilon_{n}\right|^{2}+\left|\eta_{n}\right|^{2}
$$

the pure Coulomb contribution

$$
\left(\frac{d \sigma}{d Q}\right)_{C}=\left|\alpha_{c}\right|^{2}+\left|\gamma_{c}\right|^{2}+\left|\beta_{c}\right|^{2}+\left|\delta_{c}\right|^{2}+\left|\varepsilon_{c}\right|^{2}+\left|\eta_{c}\right|^{2}
$$

and the interference contribution 


$$
\begin{gathered}
\left(\frac{d \sigma}{d \bar{Q}}\right)_{I}=2 \operatorname{Re}\left(\alpha_{n}{ }^{*} \alpha_{c}\right)+2 \operatorname{Re}\left({r_{n}}^{\star} \gamma_{c}\right)+2 \operatorname{Re}\left(\beta_{n}{ }^{\star} \beta_{c}\right)+ \\
2 \operatorname{Re}\left(\delta_{n}{ }^{\star} \delta_{c}\right)+2 \operatorname{Re}\left(\varepsilon_{n}{ }^{\star} \varepsilon_{c}\right)+2 \operatorname{Re}\left(n_{n}{ }^{\star} n_{c}\right)
\end{gathered}
$$

In this experiment, the beam is polarized and thus the differential cross section is

$$
\frac{d \sigma}{d \bar{Q}}=\left(\frac{d \sigma}{d \bar{Q}}\right)_{U N}\left(1+\vec{P}_{\mathrm{V}} \cdot \dot{n}\left(A_{n}+A_{C}+A_{I}\right)\right)
$$

where $\vec{F}_{b}$ is the beam polarization and $A_{n}, A_{c}$, and $A_{I}$ are the pure nuclear, pure Coulomb, and interference analyzing powers respectively.

$$
\begin{aligned}
A_{n}=\frac{2 \operatorname{Re}\left(\left(\alpha_{n}+\beta_{n}\right)^{\star}\left(1 \gamma_{n}\right)\right)}{\left(\frac{d \sigma}{d \bar{Q}}\right)_{U N}} \\
A_{c}=\frac{2 \operatorname{Re}\left(\left(\alpha_{c}+\beta_{c}\right)^{*}\left(i \gamma_{c}\right)\right)}{\left(\frac{d \sigma}{d \sigma}\right)_{U N}} \\
A_{I}=\frac{2 \operatorname{Re}\left(\left(\alpha_{n}+\beta_{n}\right)^{\star}\left(i Y_{c}\right)\right)+2 \operatorname{Re}\left(\left(\alpha_{c}+\beta_{c}\right)^{*}\left(i Y_{n}\right)\right)}{\left(\frac{d \sigma}{d \Omega}\right)_{U N}}
\end{aligned}
$$

Let us first consider the pure nuclear contribution to the cross section $(\mathrm{d} \sigma / \mathrm{d} Q)_{\mathrm{N}}$. A parameterization must be made to the nuclear amplitudes in order for the real part of the nuclear amplitude to be 
obtained from a fit of eqn.(100) to the data. A standard parameterization to use is the classical parameterizationi2:

$$
a_{n}=a_{n}(0) e^{-b_{1} q^{2} / 2}
$$

where $q$ is equal to $2 \mathrm{ksin} \theta / 2$ and $b_{1}$ is commonly known as the slope parameter. As was discussed in the previous section, a correction factor must be applied to the nuclear amplitudes if scattering off of nucled rather than nucleons is to be considered. From eqn.(88) we see that eqn.(108) must become

$$
\alpha_{n}=\alpha_{n}(0) W e^{-b_{1} q^{2} / 2}
$$

with

$$
V=\left(1-\frac{1 R^{2} \beta}{2 \varepsilon^{2}}\right)^{-1}
$$

In addition, we also paraneterize the other nuclear amplitudes in the following way. For the double spin-flip amplitudes

$$
\begin{gathered}
\left|\beta_{n}\right|^{2}+\left|\delta_{n}\right|^{2}+\left|\varepsilon_{n}\right|^{2}= \\
\left(\left|\beta_{n}(0)\right|^{2}+\left|\delta_{n}(0)\right|^{2}+\left|\varepsilon_{n}(0)\right|^{2}\right)|N|^{2} e^{-b_{2} q^{2}}
\end{gathered}
$$

and for the spin orbit amplitudes

$$
\begin{aligned}
& r_{n}=\left(r_{1}+i r_{2}\right) \sin \theta e^{-b_{3} q^{2 / 2}} \\
& \eta_{n}=\left(n_{1}+i \eta_{2}\right) \sin \theta e^{-b_{4} q^{2 / 2}}
\end{aligned}
$$


where $r_{1}, r_{2}, n_{1}, r_{2}, b_{3}$, and $b_{4}$ are parameters to be determined from fitting the data. Combining eqns.(101), (109), (111), and (112) we have

$$
\begin{aligned}
& \left(\frac{d \sigma}{d \bar{Q}}\right)_{N}=\left|a_{n}(0)\right|^{2}|u|^{2}\left(e^{-b_{1} q^{2}}+R e^{-b_{2} q^{2}}\right)+ \\
& \left(s_{1} e^{-b_{3} q^{2}}+s_{2} e^{-b_{4} q^{2}}\right) \sin ^{2} \theta
\end{aligned}
$$

where we have set

$$
R=\frac{\left|\beta_{n}(0)\right|^{2}+\left|\delta_{n}(0)\right|^{2}+\left|\varepsilon_{n}(0)\right|^{2}}{\left|\alpha_{n}(0)\right|^{2}}
$$

and

$$
\begin{aligned}
& s_{1}=r_{1}^{2}+r_{2}^{2} \\
& s_{2}=n_{1}^{2}+n_{2}^{2}
\end{aligned}
$$

Ve note that

$$
\begin{aligned}
\left|\alpha_{n}(0)\right|^{2} & =\left(\operatorname{Im\alpha _{n}}(0)\right)^{2}+\left(\operatorname{Re} \alpha_{n}(0)\right)^{2} \\
& =\left(\operatorname{Im\alpha _{n}}(0)\right)^{2}\left(1+\alpha^{2}\right)
\end{aligned}
$$

where $\alpha$ is the ratio of the real to 1maginary spin independent amplitudes at zero degrees. Finally, using the optical theorem

$$
\operatorname{Im} \alpha_{n}(0)=\frac{k}{4 \pi} \sigma_{t o t}
$$


ve combine eqns.(113) and (115) to get

$$
\begin{aligned}
\left(\frac{d \sigma}{d \alpha}\right)_{N}= & \frac{k^{2}}{16 \pi^{2}} \sigma_{t o t}^{2}\left(1+\alpha^{2}\right) \mid w_{1} 2\left(e^{-b_{1} q^{2}}+R e^{-b_{2} q^{2}}\right) \\
& +\left(s_{1} e^{-b_{3} q^{2}}+s_{2} e^{-b_{4} q^{2}}\right) \sin ^{2} \theta
\end{aligned}
$$

For the Coulomb contribution to the differential cross section we consider only $\alpha_{c}$ and $r_{c}$ as the other coulomb amplitudes are negligible in comparison. The spin independent amplitude is therefore equal to the first half of eqn. (88)

$$
\alpha_{c}=-\frac{\beta}{2 k \sin ^{2} \theta / 2} e^{21 \phi}
$$

The spin orbit amplitude is given by

$$
r_{c}=-1 \sin \theta \frac{\hbar^{2} k^{2}}{2 \mu^{2} c^{2}} \alpha_{c}\left(\mu-\frac{1}{2}\right)
$$

where $M$ is the aass of the proton and $\mu$ is the proton magnetic moment ( $\mu=2.79$ nuclear magneton). Using the two expressions one finds that the Coulomb contribution to the differential cross section is

$$
\begin{aligned}
& \left(\frac{\partial \sigma}{d \alpha}\right)_{C}=\left|\alpha_{c}\right|^{2}+\left|\gamma_{c}\right|^{2} \\
= & \frac{\beta^{2}}{4 k^{2} \sin ^{4} \theta / 2}\left(1+T^{2} \sin ^{2} \theta\right)
\end{aligned}
$$


where

$$
T=\frac{\hbar^{2} k^{2}}{2 M^{2} c^{2}}\left(\mu-\frac{1}{2}\right)
$$

The interference term, eqn.(103) will then be

$$
\begin{aligned}
& \left(\frac{d \sigma}{d Q}\right)_{I}=2 \operatorname{Re}\left(\alpha_{n}{ }^{\star} \alpha_{c}\right)+2 \operatorname{Re}\left(\gamma_{n}{ }^{\star} \gamma_{c}\right) \\
& =2\left(\operatorname{Re}_{n} \operatorname{Re}_{c}+I m \alpha_{n} I m \alpha_{c}+\operatorname{Rer}_{n} \operatorname{Rer}_{c}+\operatorname{Im} r_{n} I m r_{c}\right)
\end{aligned}
$$

Into this expression, we put the parameterization for the nuclear amplitudes, eqns.(109) and (112), along with the formulae for the Coulomb amplitudes, eqns.(118) and (119). After some algebra we then have

$$
\begin{aligned}
& \left(\frac{d \sigma}{d \bar{Q}}\right)_{I}=-\frac{\beta}{k \sin ^{2} \theta / 2}\left(\frac{k}{4 \pi} \sigma_{\operatorname{tot}} e^{-b_{1} q^{2} / 2}(\alpha(\cos 2 \phi R e W+\sin 2 \phi I m W)\right. \\
& \left.+(\sin 2 \phi R e W-\cos 2 \phi I m W))+T \sin ^{2} \theta e^{-b_{3} q^{2} / 2}\left(r_{1} \sin 2 \phi-r_{2} \cos 2 \phi\right)\right)(1
\end{aligned}
$$

Finally, we combine the pure nuclear, pure Coulomb, and interference contributions to the elastic differentlal cross section, eqns.(117), (120), and (122) to obtain

$$
\frac{d \sigma}{d Q}=\frac{k^{2}}{16 \pi^{2}} \sigma_{t o t}^{2}\left(1+\alpha^{2}\right)|w|^{2}\left(e^{-b_{1} q^{2}}+\operatorname{Re}^{-b_{2} q^{2}}\right)
$$




$$
\begin{gathered}
+\left(s_{1} e^{-b_{3} q^{2}}+s_{2} e^{-b_{4} q^{2}}\right) \sin ^{2} \theta+\frac{\beta^{2}}{4 k^{2} \sin ^{4} \theta / 2}\left(1+T^{2} \sin ^{2} \theta\right) \\
-\frac{\beta}{k \sin ^{2} \theta / 2}\left(\frac{k}{4 \pi} \sigma_{\operatorname{tot}} e^{-b_{1} q^{2} / 2}(\alpha(\cos 2 \phi R e V+\sin 2 \phi \operatorname{Im} W)\right. \\
\left.+(\sin 2 \phi R e w-\cos 2 \phi \operatorname{Im} \psi))+\operatorname{Tsin}^{2} \theta e^{-b_{3} q^{2} / 2}\left(r_{1} \sin 2 \phi-r_{2} \cos 2 \phi\right)\right)
\end{gathered}
$$

We now have an expression for the elastic differential cross section in terms of the parameters $\sigma_{\text {tot }}, \alpha, b_{1}, b_{2}, b_{3}, b_{4}, r_{1}, r_{2}, n_{1}, n_{2}$, and $R$. Once do/de is measured, one simply fits the data points to eqn.(123) In order to obtain the values of these independent parameters. For our particular case of protons scattering off of spinless nuclei, some of the parameters in eqn.(123) can be set equal to zero. In particular, from eqn. (94) we see that since $\vec{\sigma}_{2}=0$ for spinless nuclei, $\beta_{n}, \delta_{n}$, and $\varepsilon_{n}$ are zero. Assuming charge independence, $n_{n}$ is also zero. Therefore, $b_{2}, b_{4}, n_{1}, n_{2}, R$, and $s_{2}$ will all be zero in the above expression for the differential cross section. The task is then to determine the six parameters $\sigma_{\text {tot }}, \alpha$, $b_{1}, b_{3}, r_{1}, r_{2}$

The analyzing powers, eqns.(105), (106), and (107), can also be given the same parameterization treatment as for the differential cross section. Inserting eqns.(109), (112), (118), and (119) into the expressions for the analyzing powers, and setting the $B^{\prime}$ s equal to zero (ie. $B_{n}$ and $B_{c}$ in eqns.(105), (106), and (107)), we have after some algebra 


$$
\begin{gathered}
\left(\frac{d \sigma}{d Q}\right)_{U N} A_{n}=\frac{k \sigma_{t o t}}{2 \pi} \sin \theta e^{-\left(b_{1}+b_{3}\right) q^{2} / 2 \times} \\
\left(\alpha\left(r_{1} I m W-r_{2} \operatorname{ReW}\right)+\left(r_{1} \operatorname{Re} W-r_{2} I m W\right)\right) \\
\left(\frac{d \sigma}{d Q}\right)_{U N}{ }_{C}=\frac{\beta^{2} T \sin \theta}{2 k^{2} \sin ^{4} \theta / 2} \\
\left(\frac{d \sigma}{d Q}\right)_{U N}{ }_{I}=\frac{B \sin \theta}{k \sin ^{2} \theta / 2}\left(\left(r_{2} \cos 2 \phi-r_{1} \sin 2 \phi\right) e^{-b} q_{3}^{2 / 2}\right. \\
\left.-\frac{T k \sigma_{\operatorname{tot}} e^{-b_{1} q^{2} / 2}}{4 \pi}(\alpha(\cos 2 \phi R e W+\sin 2 \phi I m W)+(\sin 2 \phi R e W-\cos 2 \phi I m W))\right)
\end{gathered}
$$

Eqn. (123) for the differential cross section together with the sum of eqns.(124), (125), and (126) for the analyzing power are then the basic expressions to be used in extracting the six parameters from the data. From the values of these parameters, some of the theoretical interpretations can be tested. In the next section, we will look at one of these theories.

\section{II-D. DISPERSION RELATIONS}

Once the real part of the forward nuclear amplitude is measured, the natural thing to do is to see how the experimental value compares with theory. The most common theory to use, and indeed one of the most successful theories in dealing with $\operatorname{Ref}_{n}$, is the dispersion relation approach ${ }^{13}$. Forvard dispersion relations are highly useful because they allow the mesonic and antinucleon degrees of freedom in 
elastic scattering to surface so that their contributions to the amplitude can be studied. In addition, the properties of the deeply bound nuclear states of nuclei can be investigated since they show up in the exchange amplitude which is a major contributor to forward dispersion relations for nucleon-nucleus scattering.

Dispersion relations are more commonly used in particle-particle scattering than in nucleon-nucleus scattering for essentially two reasons. First of all there is much more experimental data existing for the former than for the later. And secondly, it is much easter to apply dispersion relations to the particle-particle case than it is to apply them to nucleon-nucleus scattering because of the many bound and excited states of nuclei that can lead to large numbers of poles and cuts. However, the complication arising from these singularities can be greatly reduced by replacing the many poles and branch cuts by a few effective poles along the path of integration, and sometimes even one effective pole is adequate. This is analogous to replacing the Hamiltonian describing a particle of mass $m$ in a central potential $V(r)$ by one describing a particle of reduced mass $u$ in an effective potential

$$
V_{e f f}(r)=\frac{L^{2}}{2 \mu r^{2}}+V(r)
$$

in order to simplify the problem. Thus the difficulty introduced by the many poles and cuts in nucleon-nucleus scattering can be dealt with. One need not abandon the use of dispersion relations in favor 
of other supposedly easier to use scattering formalisms which are incapable of probing the nuclear properties mentioned above on the grounds that dispersion relations make tedious calculations.

The general dispersion relation is easily obtained from Cauchy's integral formula

$$
f(z)=\frac{1}{2 \pi i} \int_{C} \frac{f\left(z^{\prime}\right) d z^{\prime}}{z^{\prime}-z}
$$

If we take $f$ to be the forward scattering amplitude as a function of the total energy $K$, with $K$ complex, then

$$
f(K)=\frac{1}{2 \pi i} \int_{c} \frac{f\left(K^{\prime}\right) d K^{\prime}}{K^{\prime}-K^{\prime}}
$$

Since $f(K)$ is analytic in the upper half $K$ plane by causality, the contour of the integral in eqn. (128) is chosen to be

$$
\int_{c}=\int_{-\infty}^{\infty}+\int_{\text {semicircle }}
$$

as shown in figure 1 . The contribution from the large semicircle at infinity vanishes since $f(K) \rightarrow 0$ as $|K| \rightarrow-$. He are left then with the integration along the real energy axis $E$ which gives

$$
2 \pi i f(E)=\int_{-\infty}^{\infty} \frac{f\left(E^{\prime}\right) d E^{\prime}}{E^{\prime}-\bar{E}}+\pi i f(E)
$$

or 


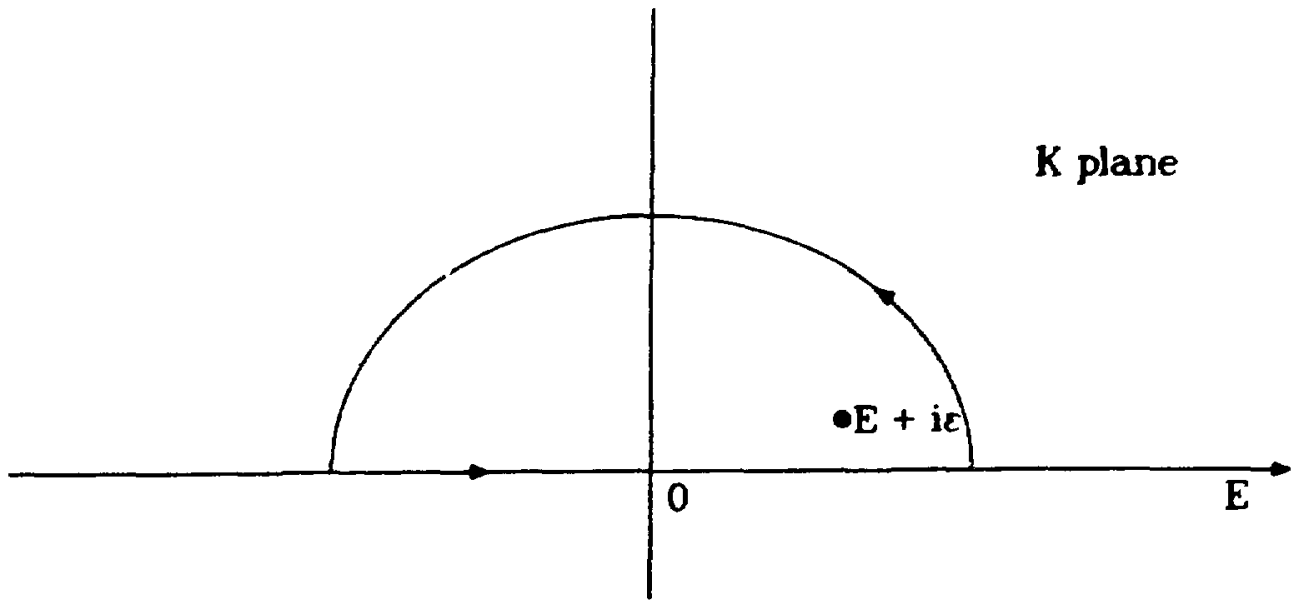

F1g. 1

The contour of the integral of eqn. (128) in the complex $K$ plane.

$$
f(E)=\frac{1}{\pi I} \int_{-\infty}^{\infty} \frac{f\left(E^{\prime}\right) d E^{\prime}}{\bar{E}^{\prime}-E}
$$

Equating real and imaginary parts yields

$$
\operatorname{Re} f(E)=\frac{1}{\pi} \int_{-\infty}^{\infty} \frac{\operatorname{Imf}\left(E^{\prime}\right) \mathrm{d} E^{\prime}}{E^{\prime}-E}
$$

This is the general dispersion relation relating the imaginary and real parts of the forward scattering anplitude. The problem of calculating Ref(E) reduces to measuring or calculating Imf(E) at all energles, physical and unphysical, in the integrand of eqn.(132).

In what follows, we will discuss the particular case of $p-{ }^{40} \mathrm{Ca}$ forvard scattering. Ue note first from the general dispersion relation eqn.(132) that there are three distinct regions of integration. These are normal nucleon scattering for $E>m_{p}$ ' 
antinucleon scattering for $E<-m_{p}$, and the unphysical region $-m_{p}<E$ $<m_{p}$. We will discuss each of these regions separately as the treatment for each is different.

For $E>m_{p}, \operatorname{Im} f(E)$ is determined from the optical theorem and so it is directly measurable. The major difficulty comes from the fact that the integrand of eqn.(132) is not convergent. This problem is circumvented by subtracting the dispersion relaition at some energy $E_{0}$ so that

$$
\operatorname{Ref}(E)-\operatorname{Ref}\left(E_{0}\right)=\left(E-E_{0}\right) \frac{1}{\pi} \int_{-\infty}^{\infty} \frac{\operatorname{Imf}\left(E^{\prime}\right) d E^{\prime}}{\left(E^{\prime}-E_{0}\right)\left(E^{\prime}-E\right)}
$$

which has a convergent integrand. $E_{0}$ can be chosen to be anything, but it should be chosen at a place where Ref $\left(E_{0}\right)$ is known. A convenient choice is $E=m_{p}$, the threshold for scattering.

To evaluate the part of the integrand for $E<-m_{p}$ where antinucleon scattering takes place, we use the crossing relation between nucleon and antinucleon amplitudes to change the region of integration from $E<-m_{p}$ to $E>m_{p}$, namely

$$
\operatorname{Imf}(-E)=-\operatorname{Im} \bar{f}(E)
$$

where $\bar{f}$ is the forward antinucleon scattering amplitude. Thus

$$
\int_{-\infty}^{-m} \frac{\operatorname{Imf}\left(E^{\prime}\right) d E^{\prime}}{E^{\prime}-E}=\int_{m}^{\infty} \frac{\operatorname{Im} \bar{f}\left(E^{\prime}\right) d E^{\prime}}{E^{\prime}+\bar{E}}
$$


The manifestation of the antinucleon amplitude in the dispersion relation allows the relationship between nucleon and antinucleon processes to be studied. The two processes are in fact quite similar as is evident from the graphs of figures $2-5$. Figure 2 shows the direct or $s$ channel for proton scattering on ${ }^{40} \mathrm{Ca}$. Mex is the mass of the exchanged nucleus or nuclei which may be excited. The equivalent diagram for antiproton scattering is shown in fig. 3 .

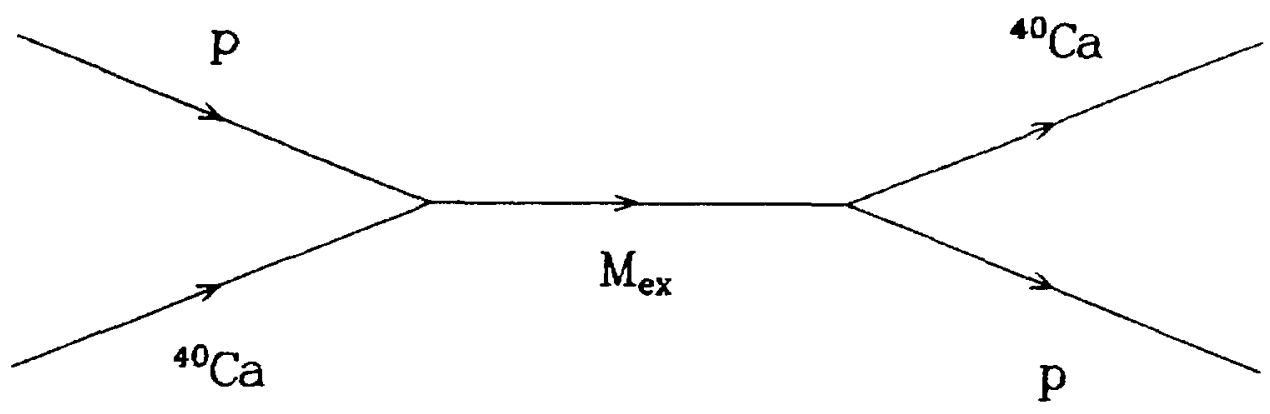

Fig. 2

The direct $s$ channel for proton scattering on ${ }^{40} \mathrm{Ca}$.

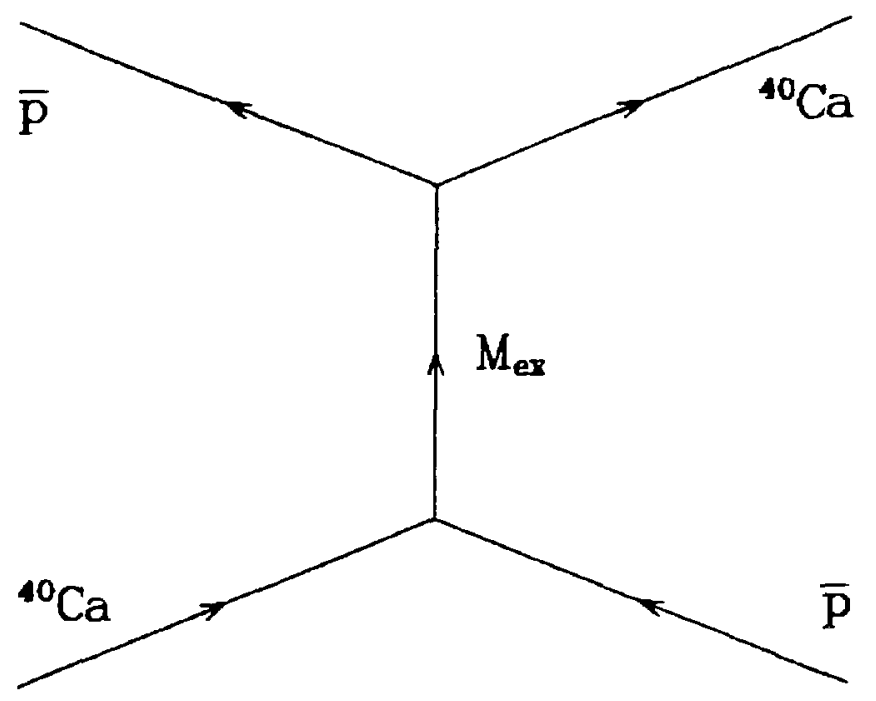

Fig. 3

The equivalent diagram of figure 2 for antiproton scattering. 


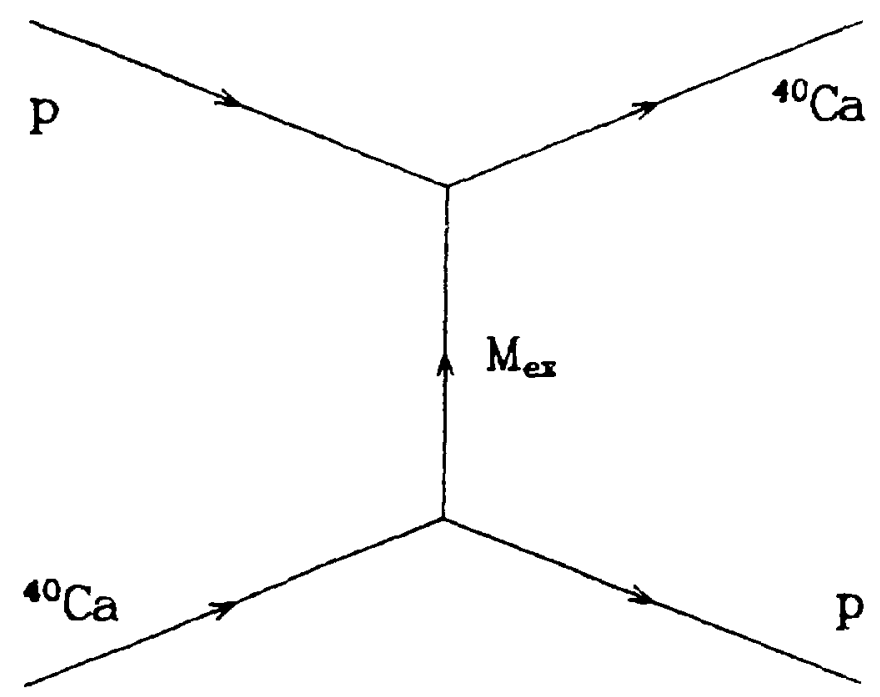

Fig. 4

The exchange $u$ channel for proton scattering on ${ }^{40} \mathrm{Ca}$.

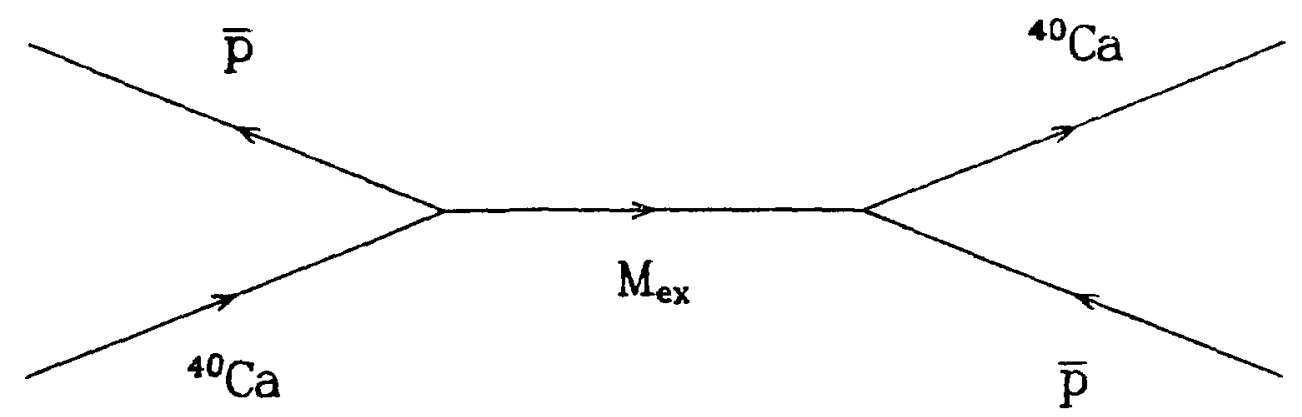

Fig, 5

The equivalent diagram of figure 4 for antiproton scattering.

Similarly fig. 4 shows the exchange or $u$ channel for proton scattering while fig. 5 is the corresponding antiproton scattering diagram. These four diagrams are the basic diagrams that not only aid In understanding processes in the physical region of the dispersion relation, but also allow calculation of the poles and cuts In the unphysical region $|E|<m_{p}$. And, as we shall see, the 
exchange channel diagrams play the most important role in the unphysical region.

Performing the integration of eqn.(132) in the unphysical region is of course the most challenging part since experiments do not give the value of $\operatorname{Imf}(E)$ in this region. One can, however, analytically continue an expansion of $\operatorname{Imf}(E)$ in the physical region into the unphysical region near the threshold for scattering. But far below the threshold, the poles and cuts need to be evaluated. Poles arise in the direct channel when

$$
s=\left(P_{p}+P_{C a}\right)^{2}=M_{e x}^{2}
$$

where $P_{p}$ and $P_{C a}$ are the four momenta of the incident proton and calcium nuclei respectively. Similarly, in the exchange channel the condition for poles is

$$
u=\left(P_{p}-P_{C a}\right)^{2}=M_{e x}^{2}
$$

Evaluating eqns.(136) and (137) gives us the position of the poles in the unphysical region at energies

$$
\begin{gathered}
E_{\text {pole }}= \pm \frac{M_{e x}^{2}-m_{p}^{2}-H_{C a}^{2}}{2 M_{C a}}, \\
\left|E_{p o l e}\right|<m_{p}
\end{gathered}
$$

where the + sign is for the direct channel and the - sign for the 
exchange channel. In the exchange channel for $\mathrm{p}-4^{40} \mathrm{Ca}$ scattering there are many poles corresponding to $M_{e x}$ equal to the mass of the exchanged nucleus ${ }^{39} \mathrm{~K}$ or one of 1 ts excited states. A few of these are listed in table 1. The direct channel has only one pole in the unphysical region which occurs for $M_{\text {ex }}$ equal to the mass of $41 \mathrm{Sc}$. The excited states of ${ }^{41} \mathrm{Sc}$ are all at energies above the threshold for scattering. Thus we see that exchange poles contribute much more significantly than direct poles to the dispersion relation in the unphysical region.

In addition to poles, there are also branch cuts in the unphysical region. These cuts arise when ${ }$ ex in the direct or exchange channel consists of two or more particles. Since these particles can have any amount of relative kinetic energy between them, the exact position of the singularity is obscure. Thus these singularities contribute as branch cuts with the branch point given by the position of zero relative kinetic energy of the intermediate

Table 1 - Exchange poles for $\mathrm{P}-{ }^{40} \mathrm{Ca}$ scattering.

\begin{tabular}{cccc}
\hline$J^{P}$ & Excitation & $E_{\text {pole }}(\mathrm{HeV})$ & $T=E_{p o l e^{-m_{p}}(\mathrm{MeV})}$ \\
\hline $3 / 2^{+}$ & 0 & 930.74 & -8.06 \\
$1 / 2^{+}$ & 2.5226 & 928.28 & -10.52 \\
$7 / 2^{-}$ & 2.8138 & 927.99 & -10.81 \\
$3 / 2^{-}$ & 3.0193 & 927.80 & -11.00 \\
$9 / 2^{-}$ & 3.5976 & 927.23 & -11.57 \\
$3 / 2,5 / 2^{-}$ & 3.8831 & 926.95 & -11.85 \\
$\vdots$ & $\vdots$ & $\vdots$ & $:$ \\
$(3 / 2,5 / 2)^{+}$ & 6.631 & 924.56 & -14.24 \\
\hline
\end{tabular}


state particles. In $\mathrm{p}-{ }^{40} \mathrm{Ca}$ scattering, there are no branch cuts in the direct channel because the nuclear binding does not permit two or more particle intermediate states with $\mathrm{M}_{\text {ex }}<m_{p}+\mathrm{M}_{\mathrm{Ca}}$. But for the exchange channel there are many two particle intermediate states contributing to the dispersion relation in the unphysical region. Some of these are listed in table 2. Those intermediate states consisting of nuclei contribute non-relativistically while those involving mesons are relativistic. Figures 6 and 7 illustrate the relativistic exchange processes. Meson exchange appears most significantly at energies in the range of 100-1000 MeV. Thus measuring the real part of the scattering amplitude in this energy region can shed light on the meson aspect of nuclear interactions.

Collecting all the poles and cuts in the direct and exchange channel gives us a picture as shown in figure 8 . Moving left from the physical nucleon scattering region we first come upon the one direct channel pole at $E=937.64 \mathrm{MeV}$ and then a series of exchange

Table 2 - Two particle intermediate states in $\mathrm{p}-{ }^{40} \mathrm{Ca}$ scattering.

$$
\text { State } \quad E_{\text {pole }}(\mathrm{MeV}) \quad T=E_{\text {pole }}{ }^{-m_{p}(\mathrm{MeV})}
$$

$\begin{array}{lll}\mathrm{p},{ }^{38} \mathrm{Ar} & 924.53 & -14.27 \\ { }^{4} \mathrm{He},{ }^{35} \mathrm{Cl} & 923.72 & -15.08 \\ \mathrm{n},{ }^{38} \mathrm{~K} & 917.95 & -20.85 \\ { }^{19} \mathrm{~F},{ }^{20} \mathrm{Ne} & 906.10 & -32.70 \\ \mathrm{n}^{\circ},{ }^{39} \mathrm{~K} & 798.90 & -139.90\end{array}$




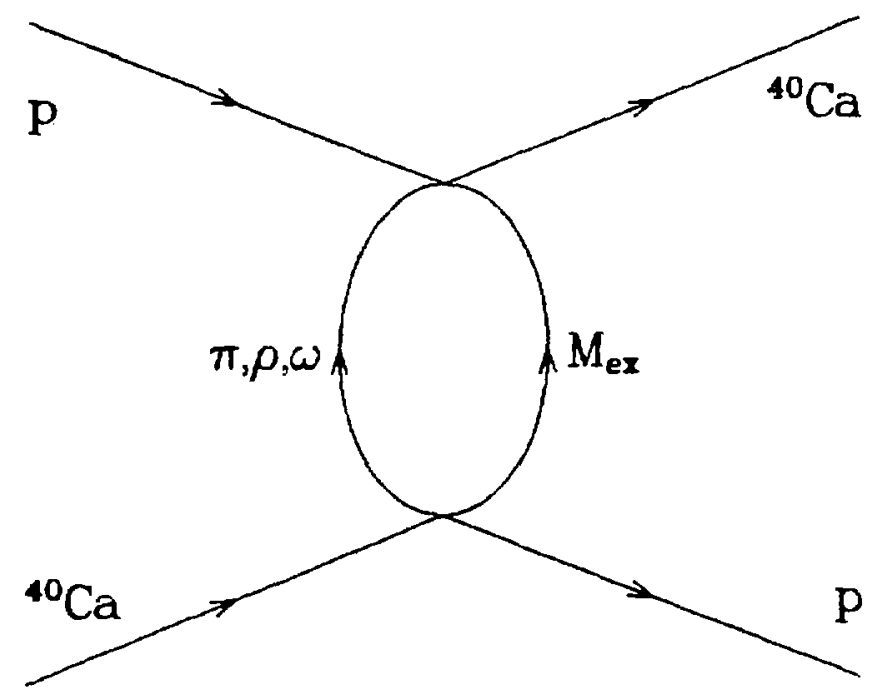

Fig. 6

Relativistic exchange processes for proton scattering.

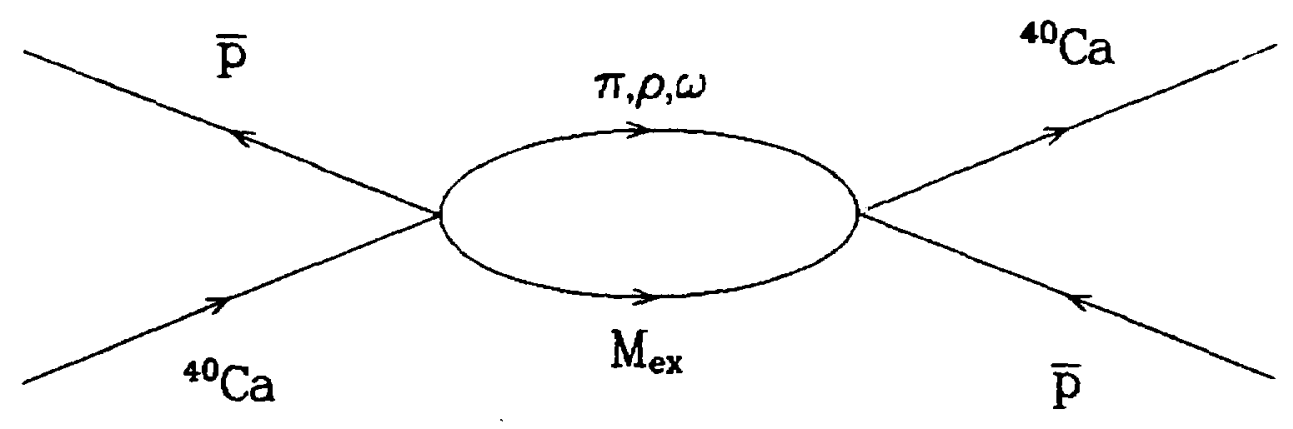

Fig. 7

Relativistic exchange processes for antiproton scattering.

poles from table 1. Two particle exchange sets in at $E=924.53 \mathrm{MeV}$ corresponding to a $\mathrm{p},{ }^{38_{\mathrm{Ar}}}$ intermediate state of zero relative kinetic energy. The branch cut for this exchange extends to the left with other branch cuts for two particle intermediate states shown at the energies where they appear as branch points. Not all the multi particle intermediate states are shown and table 2 is by no means an exhaustive list of such states. It is clear from this discussion 
that there can be many branch cuts due to many exchange processes. Knowledge of Ref $f_{n}$ therefore provides a way of studying these processes through dispersion relations.

Having examined the different energy regions of integration in the integrand of eqn.(132), we write the general dispersion relation In a more useful form. Instead of dividing the energy regions at the physical and unphysical boundaries, the dispersion relation is broken into the segments as suggested by figure $B$. Namely, $-\infty<E<E_{h b}$ with $E_{h b}$ the energy of the highest branch point, in this case $E_{h b}=$ $924.53 \mathrm{HeV}, E_{h b}<E<m_{p}$ or the unphysical region containing the poles, and $E>m_{p}$. Eqn. (132) then becomes

$$
\operatorname{Ref}(E)=\frac{1}{\pi} \int_{-\infty}^{E_{h b}} \frac{\operatorname{Imf}\left(E^{\prime}\right) d E^{\prime}}{E^{\prime}-E}+\sum_{\text {poles }} \frac{r_{p o l e}}{E_{p o l e}-E}+\frac{1}{\pi} \int_{m_{p}}^{\infty} \frac{\operatorname{Imf}\left(E^{\prime}\right) d E^{\prime}}{E^{\prime}-E}
$$

where $r_{p o l e}$ is the residue of a pole in the region $E_{h b}<E<m_{p}$ and the sum is over all poles in the region. Using the fact that

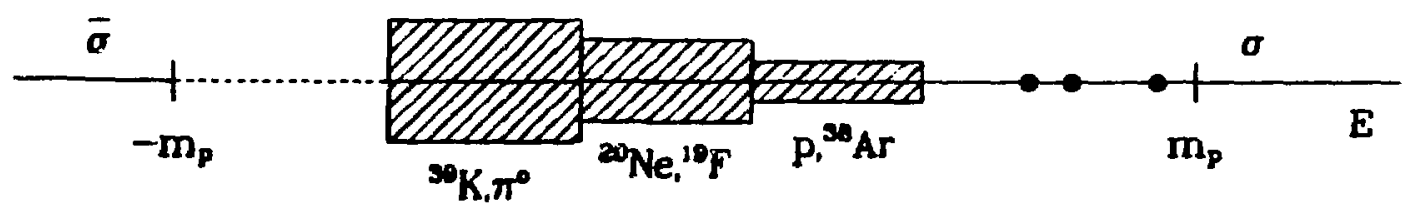

Fig. 8

Poles and branch cuts for $\mathrm{p}-{ }^{40} \mathrm{Ca}$ scattering.
. 


$$
\int_{-\infty}^{E_{h b}} \frac{\operatorname{Im} f\left(E^{\prime}\right) d E^{\prime}}{E^{\prime}-\bar{E}}=\int_{-E_{h b}}^{\infty} \frac{\operatorname{Im} \bar{f}\left(E^{\prime}\right) d E^{\prime}}{E^{\prime}+\bar{E}}
$$

eqn. (139) is rewritten as

$$
\begin{gathered}
\operatorname{Ref}(E)=\frac{1}{\pi} \int_{-E_{h b}}^{m p} \frac{\operatorname{Im} \bar{f}\left(E^{\prime}\right) d E^{\prime}}{E^{\prime}+E}+\sum_{p o I e s} \frac{r_{p o l e}}{E_{p o l e}-\bar{E}}+\frac{2}{\pi} \int_{m_{p}}^{\infty} \frac{E^{\prime} \operatorname{Imf}\left(E^{\prime}\right) d E^{\prime}}{E^{\prime 2}-E^{2}} \\
+\frac{1}{\pi} \int_{m_{p}}^{\infty} \frac{\operatorname{Im} \bar{f}\left(E^{\prime}\right)-\operatorname{Imf}\left(E^{\prime}\right)}{E^{\prime}+E} \mathrm{dE}^{\prime}
\end{gathered}
$$

The third term on the right-hand side of this expression does not converge and so a subtraction is necessary. Subtracting the dispersion relation at threshold gives

$$
\begin{aligned}
& \operatorname{Ref}(E)-\operatorname{Ref}\left(m_{p}\right)=\frac{E-m_{p}}{\pi} \int_{-m_{p}}^{E_{h b}} \frac{\operatorname{Inf}\left(E^{\prime}\right) d E^{\prime}}{\left(E^{\prime}-E\right)\left(E^{\prime}-m_{p}\right)}
\end{aligned}
$$

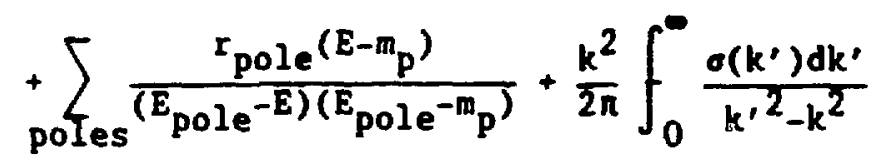

$$
\begin{aligned}
& +\frac{m_{p}-E}{4 \pi^{2}} \int_{m_{p}}^{\infty} \frac{\left(\bar{\sigma}\left(E^{\prime}\right)-\sigma\left(E^{\prime}\right)\right) k^{\prime} d E^{\prime}}{\left(E^{\prime}+m_{p}\right)\left(E^{\prime}+E\right)}
\end{aligned}
$$

where we have used the optical theorem to express the last two terns in terms of the total cross section. All the terms in this equation are now convergent, convergence for the last term resulting from the Pomeranchuk theorem. The first two terms on the right side are unphysical contributions while the other two terms are over the 
physical region and can thus be measured. As mentioned previously, the poles and cuts in the unphysical region can be replaced by a few effective poles. The procedure for doing this is to 1solate the unphysical contribution by comparing eqn.(142) with the real amplitude obtained from a phase shift analysis. Namely,

$$
\text { Ref }_{\text {unphy }}=\operatorname{Ref}_{\text {phase }}-\operatorname{Ref}_{\text {phy }}
$$

where Ref unphy is the unphysical amplitude equivalent to the first two terms on the right side of eqn.(142), Ref phase is the amplitude calculated from a phase shift analysis, and Refphy is the physical amplitude that is measured. Since Ref unphy is to be expressed in terms of effective poles, we have

$$
\text { Ref unphy }=\sum_{i} \frac{r_{\text {eff }}^{i}\left(E-m_{p}\right)}{\left(E_{e f f}^{i}-E\right)\left(E_{e f f}^{1}-m_{p}\right)}
$$

where the sum is over effective poles, $r_{e f f}^{i}$ is the residue of the $i$ th effective pole and $E_{e f f}^{1}$ is the $i$ th effective pole energy. It must be noted that this sum contains considerably fewer terms than the sum over pcles in egn.(142). Indeed, for $\mathrm{p}={ }^{40} \mathrm{Ca}$ scattering, the number of poles summed in eqn.(142) is of the order of 40, while eqn.(144) may have just 1 or 2 terms. In addition, the cuts in the first term on the right side of eqn.(142) are completely absorbed into eqn.(144). Clearly, utilizing eqn.(144) is highly advantageous.

To find the effective pole residues and energies, a plot of 
$\left(E-m_{p}\right) /\left(\right.$ Ref $_{\text {phase }}-$ Ref $\left._{\text {phy }}\right)$ vs. E is implemented. Such a plot describes a line of the form

$$
\frac{\left(E-m_{p}\right)}{\operatorname{Ref}_{\text {phase }}-\operatorname{Ref}{ }_{p h y}}=\sum A_{1} E_{\text {eff }}^{1}-E \sum_{i} A_{i}
$$

with

$$
A_{1}=\frac{E_{\text {eff }}^{i} m_{p}}{r_{\text {eff }}^{i}}
$$

Thus one can simply read the parameters from the plot.

$$
\text { Horizontal intercept at } E=\frac{\sum_{A_{1} E_{\text {eff }}^{1}}}{\sum_{\text {slope }}=-\sum_{i} A_{1}}
$$

If only one effective pole is necessary, the line should cross the $E$ axis at a value close to one of the poles in table 1 . This means that that pole is the main pole contributing to the dispersion relation in the unphysical region. Any small discrepancy between the effective pole value from the plot and a value in table 1 should be explained by a second effective pole. The energy of the second effective pole is best obtained by setting the energy of the first effective nole to the value from table 1 that it vas closest to when 
assuming only one effective pole and allowing the energy of the second effective pole to vary while examining a plot of

$$
\frac{\left(E_{e f f}^{1}-E\right)\left(E_{e f f}^{2}-E\right) \operatorname{Ref} \text { unphy }}{\left(E-m_{p}\right)}
$$

vs. $E-m_{p}$. This plot should be linear for a particular value of $E_{e f f}^{2}$ when two effective poles are approximated for Refunphy. If such is not the case, a more detailed analysis consisting of additional effective poles is necessary.

Finally, it is interesting to consider the dispersion relation from a potential scattering point of view. In scattering from a nuclear potential, the dispersion relation eqn.(132) is written as two terms describing the bound states of the nucleus and the continuum:

$$
\operatorname{Ref}(E)=\sum_{b s} \frac{r_{b s}}{E_{b s^{-E}}}+\frac{1}{n} \int_{m_{p}}^{\infty} \frac{I m f\left(E^{\prime}\right) d E^{\prime}}{E^{\prime}-E}
$$

The sum is over the bound states and $r_{b s}$ and $E_{b s}$ are the residues and energies of the bound states respectively. Using the optical theorem we have

$$
\operatorname{Ref}(E)=\sum_{b s} \frac{r_{b s}}{E_{b s^{-E}}-\frac{1}{2 \pi^{2}}} \int_{0}^{\infty} \frac{k^{\prime 2} \sigma\left(k^{\prime}\right) d k^{\prime}}{k^{\prime 2}-k^{2}}
$$


The problem with potential scattering is that for a potential satisfying the Schrbidinger equation

$$
\left(\nabla^{2}+k^{2}\right) \psi(\vec{r})=U(\vec{r}) \psi(\vec{r})
$$

the production and annihilation of particles cannot be included. The total cross section goes to zero at high energies in contradiction to experiment. Therefore a subtraction to eqn.(150) is necessary, subtraction in the $11 \mathrm{mit} E \rightarrow \infty$. The subtraction we use is the Born approximation ${ }^{2}$, or

$$
f(q)=-\frac{1}{4 \pi} \int e^{i \vec{q} \cdot \vec{r}_{U}} U(\vec{r}) d^{3} \vec{r}
$$

For forward scattering ve have

$$
\operatorname{Ref}(0)=-\frac{1}{4 \pi} \int U(\vec{r}) d^{3} \vec{r}
$$

with $U(\vec{r})$ real. Subtracting this from eqn.(150) gives a dispersion relation much more consistent with experiment

$$
\operatorname{Ref}(E)=\sum_{b s} \frac{r_{b s}}{E_{b s^{-E}}}+\frac{1}{2 \pi^{2}} \int_{0}^{\infty} \frac{k^{\prime 2} \sigma\left(k^{\prime}\right) d k^{\prime}}{k^{\prime 2}-k^{2}}-\frac{1}{4 \pi} \int U(\vec{z}) d^{3} \vec{z}
$$

This expression is still incapable of revealing mesonic and antinucleon properties as does eqn.(142), but it allows a 
determination of the volume integral of the potentlal, something that can be used to test various models.

We conclude from the discussions in this section that measuring the real part of the. forward nuclear amplitude has many applications arising from a dispersion relation approach. Such things as nuclear potentials, bound states in nuclei, and meson effects can be studied using dispersion relations as an analytic tool. Unfortunately, the data necessary to investigate these properties using this method is scarce. It is hoped that more interest will be generated to measure Ref $_{n}$ for scattering off of a variety of nuclel, particularly in the energy range 100-1000 MeV where mesonlc information can be obtained. 


\section{THE EXPERIMENT}

In this chapter, we discuss the apparatus used in obtaining the data presented in this work. Each of the processes involved, from accelerating the polarized proton beam to putting the data on tape are described here. The analysis of the data is discussed in the next chapter.

\section{III-A. THE POLARIZED PROTON BEAM}

Three beams are available at LAMPF: protons $\left(\mathrm{H}^{+}\right)$, negative hydrogen lons $\left(\mathrm{H}^{-}\right)$, and polarized hydrogen fons $\left(\mathrm{P}^{-}\right)$. These beams originate at ion sources inside the high-voltage terminals of $750-\mathrm{keV}$ Cockcroft-Walton type generators or preaccelerators 1,15 . Figure 9 shows this arrangement. A $P^{-}$beam is produced in stages before the preacceleration process as follows. First, a duoplasmatron with an expansion cup produces $\mathrm{H}^{+}$ions which are then passed through a cesium vapor. The cesium vapor converts the $\mathrm{H}^{+}$ions into a metastable atomic beam of neutral hydrogen ions by adding electrons to the ions in a charge-transfer process. Upon exiting the cesium vapor, only those hydrogen atoms which picked up one and only one electron are kept. The result, then, is a neutral hydrogen beam made up of several different spin states.

In order to get a polarized beam, a particular spin state of the neutral hydrogen beam must be chosen. This is accomplished through a process which quenches all spin states except one to the ground 


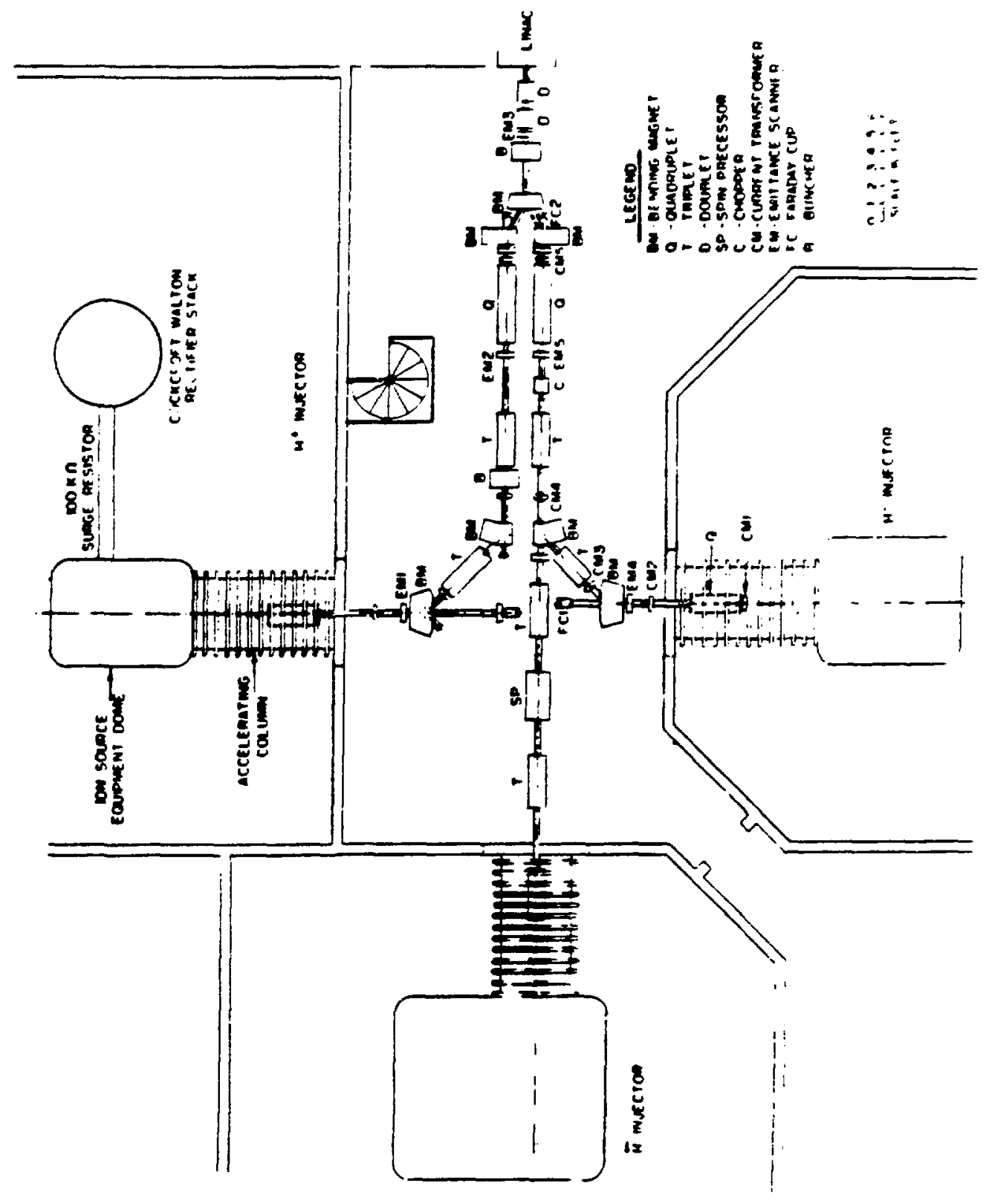

Fig. 9

Ion sources for the $\mathrm{H}^{+}, \mathrm{H}^{-}$, and $\mathrm{P}^{-}$beams at LAMPF. 
state. In particular, by applying a dc electric field and axial magnetic and electric fields of the right magnitudes, only $2 \mathrm{~s}$ state atoms with a certain nuclear spin do not get quenched to the ground state. The result is a mixture of polarized neutral hydrogen atoms and ground state atoms. The task is then to eliminate the ground state atoms from the polarized atoms. This is achieved to some extent by passing the beam through argon. The polarized atoms capture an electron in the argon and thus form the $P^{-}$beam. Unfortunately, a few of the ground state atoms also capture electrons, thus preventing a complete electromagnetic separation of polarized atoms and unpolarized atoms. The "polarized" beam will therefore contain a few unpolarized atoms. Hence, there is a limit to the polarization that the bean can have. Typical beam polarizations that can be attained under these conditions is about $80 \%$.

The polarized beam is then ready to be accelerated. First the beam is processed by the preaccelerator. The key components of the preaccelerator are the high-voltage terminals. Through charging a stack of capacitors in parallel and discharging them in series, a high multiplication in voltage is reached on the high-voltage terminals. Because the terminals operate at about $0.75 \mathrm{MV}$, they are supported on insulating columns and enclosed in aluminum housings to safeguard against sparking and corona. This high voltage is then used to preaccelerate the beam to $750 \mathrm{keV}$ before it can be accelerated to $800 \mathrm{MeV}$ by the linac. After the beam reaches an energy of $750 \mathrm{KeV}$, it is passed through a spin precessor where the 
polarization direction of the beam is ortented to a desired direction. The beam is then ready to be accelerated in the Inac to its maximum energy.

There are two sections of the linac that acceierate the beam. The first is a drift-tube linac and the second side-coupled-cavity linac. The drift-tube is of the Alvarez type, consisting of 165 drift tubes over a length of $61.75 \mathrm{~m}$ which accelerate the beam to 100 MeV. As the beam passes through these tubes, an if of about $200 \mathrm{MHz}$ is applied, allowing both $\mathrm{P}^{-}$and $\mathrm{H}^{+}$beams to be accelerated simultaneously. This is possible because at times when the $\mathrm{P}^{-}$beam is in the drift tubes, it is shielded from the accelerating potential for the $\mathrm{H}^{+}$beam. As the accelerating potential changes sign, the $\mathrm{H}^{+}$ beam enters the drift tubes while the $\mathrm{P}^{-}$beam enters the accelerating potential. Thus both $\mathrm{P}^{-}$and $\mathrm{H}^{+}$beams always "see" a potential of the right sign in order to be accelerated.

After the beam reaches an energy of $100 \mathrm{MeV}$, it passes through the side-coupled-cavity part of the linac. This section is made up of about 5000 accelerating cavities totalling one-half mile in length. Each successive pair of cavities is coupled by an external resonant cavity which serves to keep the phase between adjacent internal accelerating cavities $\pi / 2$ apart. Through physically distorting the external cavities, the beam can be fine tuned. The external resonant cavities receive their power from some 40 odd klystrons which provide 1-1/4 MW pulses at $805 \mathrm{MHz}$ rf. By choosing which klystrons are to be turned on or off, it is possible to accelerate both $\mathrm{H}^{+}$and $\mathrm{P}^{-}$in two different modes. In single energy 
mode, both $\mathrm{H}^{+}$and $\mathrm{P}^{-}$are accelerated on opposite phases of the accelerating $\mathrm{rf}$. The result is $\mathrm{H}^{+}$and $\mathrm{P}^{-}$beams of the same energy with a duty factor of $6 \%$. The time structure of the beam in this mode is 120 macropulses/s, each pulse 500 us in duration. These macropulses contain micropulses about 0.25 ns wide 5 ns apart. In dual energy mode, $\mathrm{H}^{+}$and $\mathrm{P}^{-}$beams are accelerated at different energies. In particular, the $\mathrm{H}^{+}$beam is accelerated at $800 \mathrm{MeV}$ to be used for pion production, while the $\mathrm{P}^{-}$beam is accelerated at any of several lower energies. This is accomplished through acceleration of two macropulses of $\mathrm{H}^{+}$followed by a single macropulse of $\mathrm{P}^{-}$, each

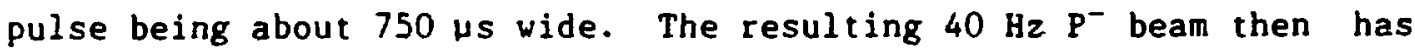
the characteristic duty factor of about 3\%. The parameters of the beams are summarized in table 3 .

After the beams have been accelerated to their final energies,

Table 3 - LAMPF Beam Parameters.

Energy
Energy Spread
Transverse Phase Space
Intensity

Pulse Structure Macropulse Length Repetition Rate

rf Microstructure

Duty Factor

$$
\begin{aligned}
& 113-800 \mathrm{MeV} \\
& \pm 0.2 \% \\
& \mathrm{n} / 3 \mathrm{mrad}-\mathrm{cm} \\
& \mathrm{H}^{+}=1 \mathrm{~mA} \text { average } \\
& \mathrm{H}^{-}=100 \mu \mathrm{A} \text { average } \\
& \mathrm{P}^{-}=0.05 \mu \mathrm{A} \text { average }
\end{aligned}
$$

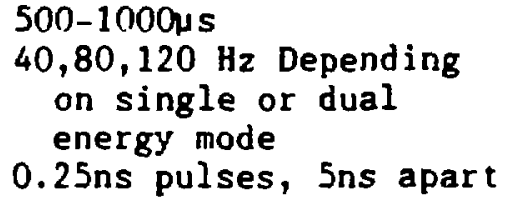

3-12\% Depending on single or dual energy mode 


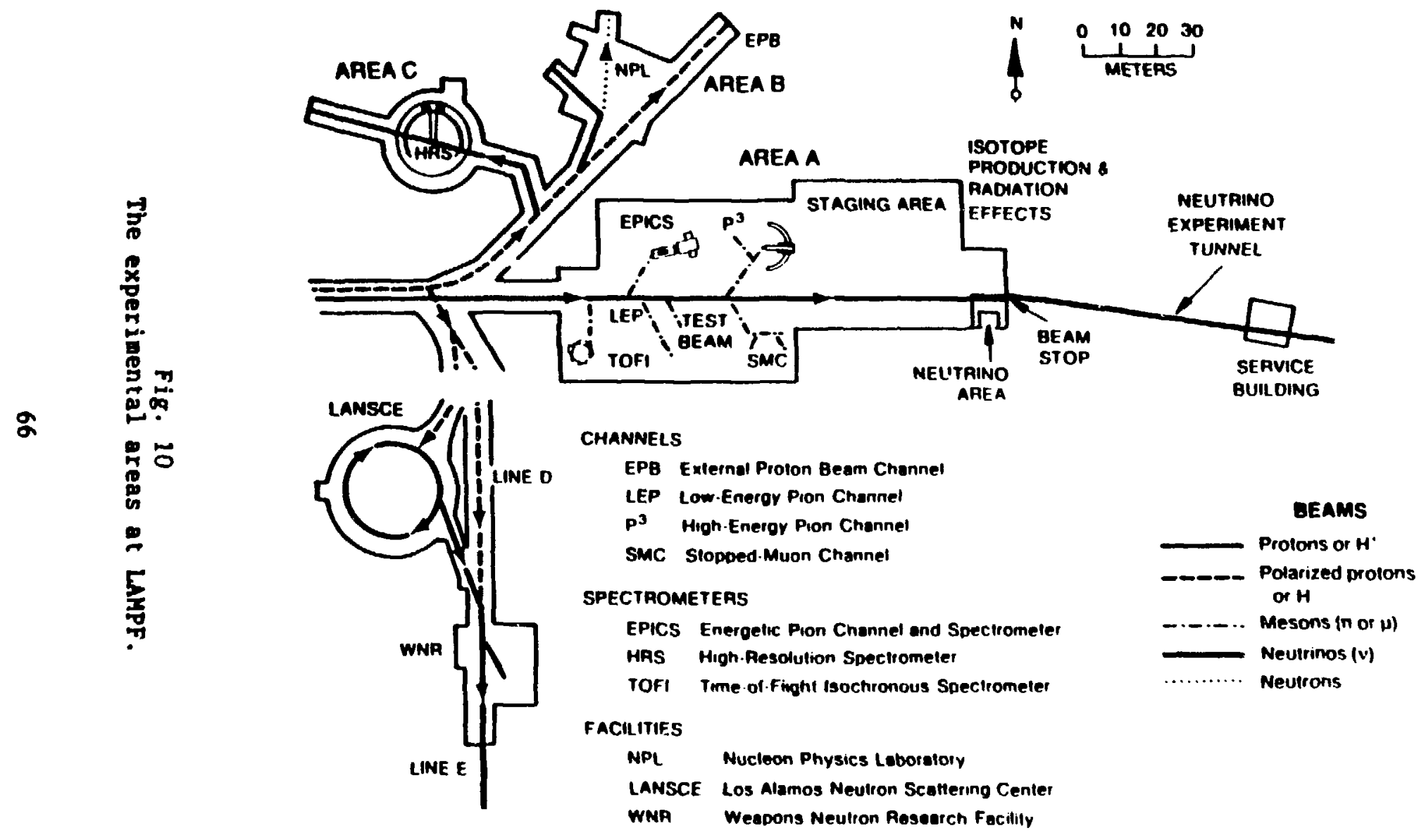


they leave the linac and enter the beam switchyard ${ }^{16}$ shown in figure 10. Here the beams are separated and focused with a variety of bending and focusing magnets. The $\mathrm{H}^{+}$beam continues straight into line $A$ while the $P^{-}$beam is bent $30^{\circ}$ northward into line $X$. A small amount of beam is also deflected southward to the Weapons Neutron Researrh Area.

In line $X$, a set of three stripper devices are used to convert a controllable amount of the $\mathrm{P}^{-}$beam to $\mathrm{P}^{+}$. These devices consist of carbon wires, aluminum ribbons and sieves, and mylar sieves of various thicknesses which control how much of the beam is to be stripped. The stripped beam $\mathrm{P}^{+}$is then deflected into line $\mathrm{C}$ while the unstripped beam $P^{-}$continues on into line $B$. Because the beam in line $C$ is controlled by the user of the High Resolution Spectrometer (HRS), the passage of the beam through line $\mathrm{C}$ is discussed together with the HRS in the next section.

III-B. LINE C AND THE HIGH RESOLUTION SPECTROMETER

Once the polarized proton beam enters line $C$, it goes through a series of quadrupole and dipole magnets ${ }^{115}$ as shown in figure 11. These magnets can be divided into four groups: 1) Separation, 2) Dispersion, 3) Matching, and 4) Twisting. As labeled in figure 11, they are: LCBMO1-3 and LCOMO1-2 for the separation group, LCBMO4-5 for the dispersion group, LCOMO3 and LCOMO9-11 for the matching group, and LCOMO4-8 for the twisting group. In the first group, the three bending magnets steer the polarized proton beam $\mathrm{P}^{+}$into line $\mathrm{C}$ 


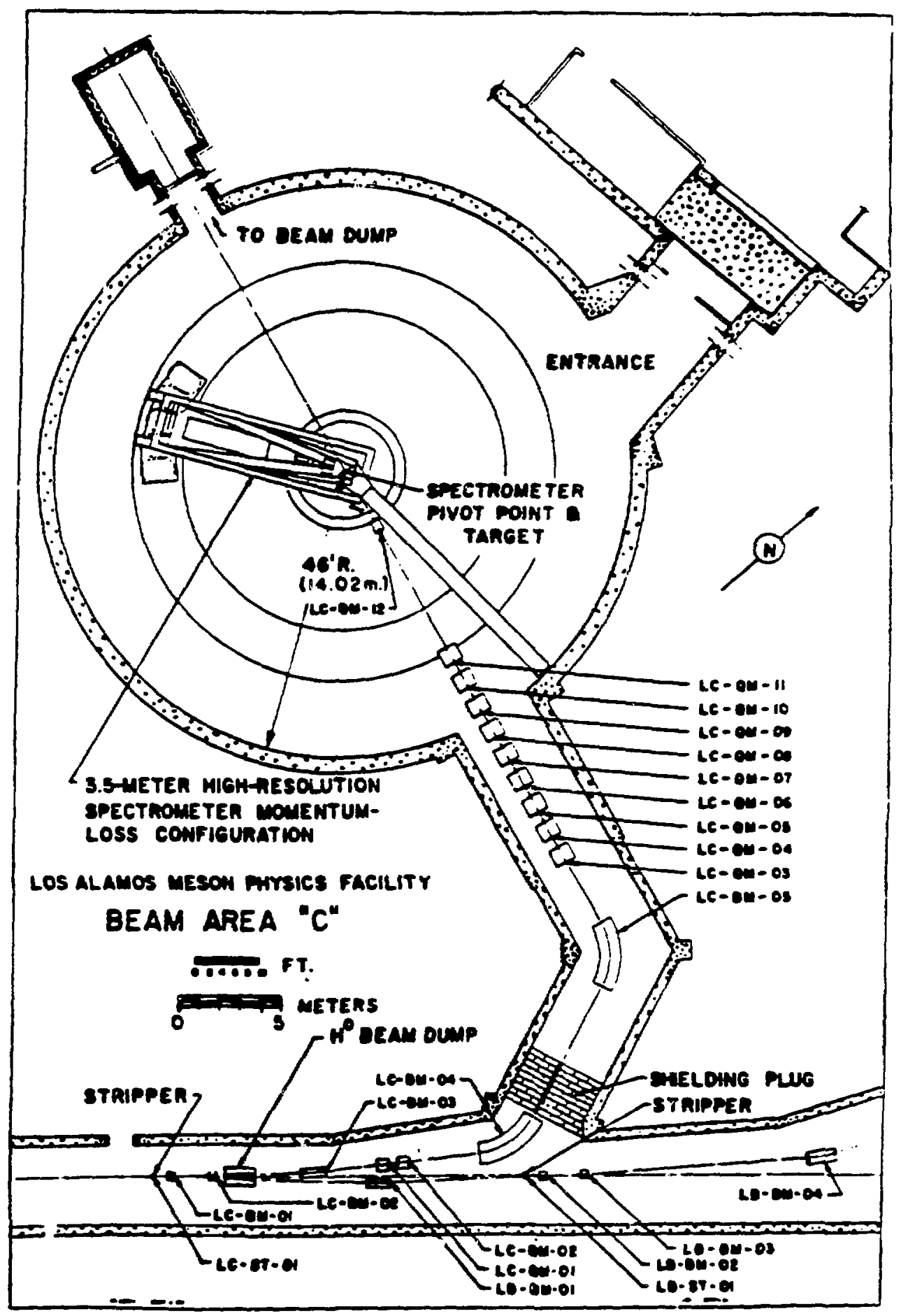

Fig. 11

Line $C$. 
avay from the residual $P^{-}$beam. The quadrupole doublet then focuses the beain into the dispersion section. The two bending magnets in the dispersion section bend the $\mathrm{P}^{+}$beam $57^{\circ}$ each and give the beam a horizontal dispersion. Next, the beam passes through overlapping matching and twisting quadrupole sections. This magnet combination focuses the beam and converts the horizontal dispersion into a vertically dispersed beam as required by the HRS acceptance for proper momentum dispersion matching.

In addition to the magnets shown in figure 11, there are also four steering magnets at various places in line $C$ used to displace the beam horizontally or vertically as desired. Sets of collimator jaws a:e also conveniently located throughout line $\mathrm{C}$ to control the intensity and phase space of the beam. Profiles of the beam can be looked at while adjusting the various magnets through ISIC's that are placed in the beam by remote control at different places in line C. Each ISIC is a small 2-plane wire chamber of active area $6.7 \times 6.7$ $\mathrm{cm}^{2}$ inside a vacuum tight enclosure. There are 25 wires in each wire plane, 14 which have $4 \mathrm{~mm}$ wire spacing and 11 in the center whict: have $1 \mathrm{~mm}$ wire spacing. The gas mixtures used for these ISIC's are He- $\mathrm{CO}_{2}$ or $\mathrm{Ar}-\mathrm{CO}_{2}$. If the beam intensity is high enough, the beam can be seen on TV monitors which show the beam hitting phosphors located near the ISIC's.

After the beam passes through all the magnets described above, it then reaches the target area. The targets are mounted on a target wheel inside a scattering chamber that is pumped down to vacuum and open to the beam line. The target wheel is rotated by remote control 
to place the desired target into the path of the beam. The targets used to obtain the data presented in this work are as follows: a ${ }^{12} \mathrm{C}$ foil of thickness $139 \mathrm{mg} / \mathrm{cm}^{2}$, a ${ }^{40} \mathrm{Ca}$ foil $73.6 \mathrm{mg} / \mathrm{cm}^{2}$ thick, and a ${ }^{208} \mathrm{~Pb}$ vertical strip $100 \mathrm{mg} / \mathrm{cm}^{2}$ thick. To avoid oxygen contamination to the calcium target, the calcium was sanded with mineral oil and kept in an argon bath until it could be placed on the target wheel and maintained in vacuum. A check was made for oxygen on this target by looking for the spectral lines characteristic of oxygen in the missing mass spectra for this target. No oxygen lines were seen, indicating that the calcium target was virtually oxygen free.

The protons that scatter off of the target in the scattering chamber can then enter the HRS, a sideview of which is seen in figure 12. The -500 ton spectrometer system rests on airpads which allow the spectrometer to rotate about a vertical axis going through the target. The HRS is a VHV energy-loss system (Vertical dispersion, Horizontal scattering, Vertical analysis). A quadrupole magnet HSOMO1 resides at the base of the spectrometer which defocuses the beam in the momentum dispersion direction, $x$, and focuses in the $y$ direction, $y$ being perpendicular to both $x$ and $z$ in a right-handed sense, with $z$ the beam direction. Two large dipole magnets, HSBM01 and HSBMO2, then bend the horizontally scattered particles $75^{\circ}$ each onto the focal plane where they are detected by a host of particle detectors. The overall focusing of the HRS is point-to-point in the dispersion direction and parallel-to-point in the $y$ direction. The general properties of the HRS are listed in table 4. 


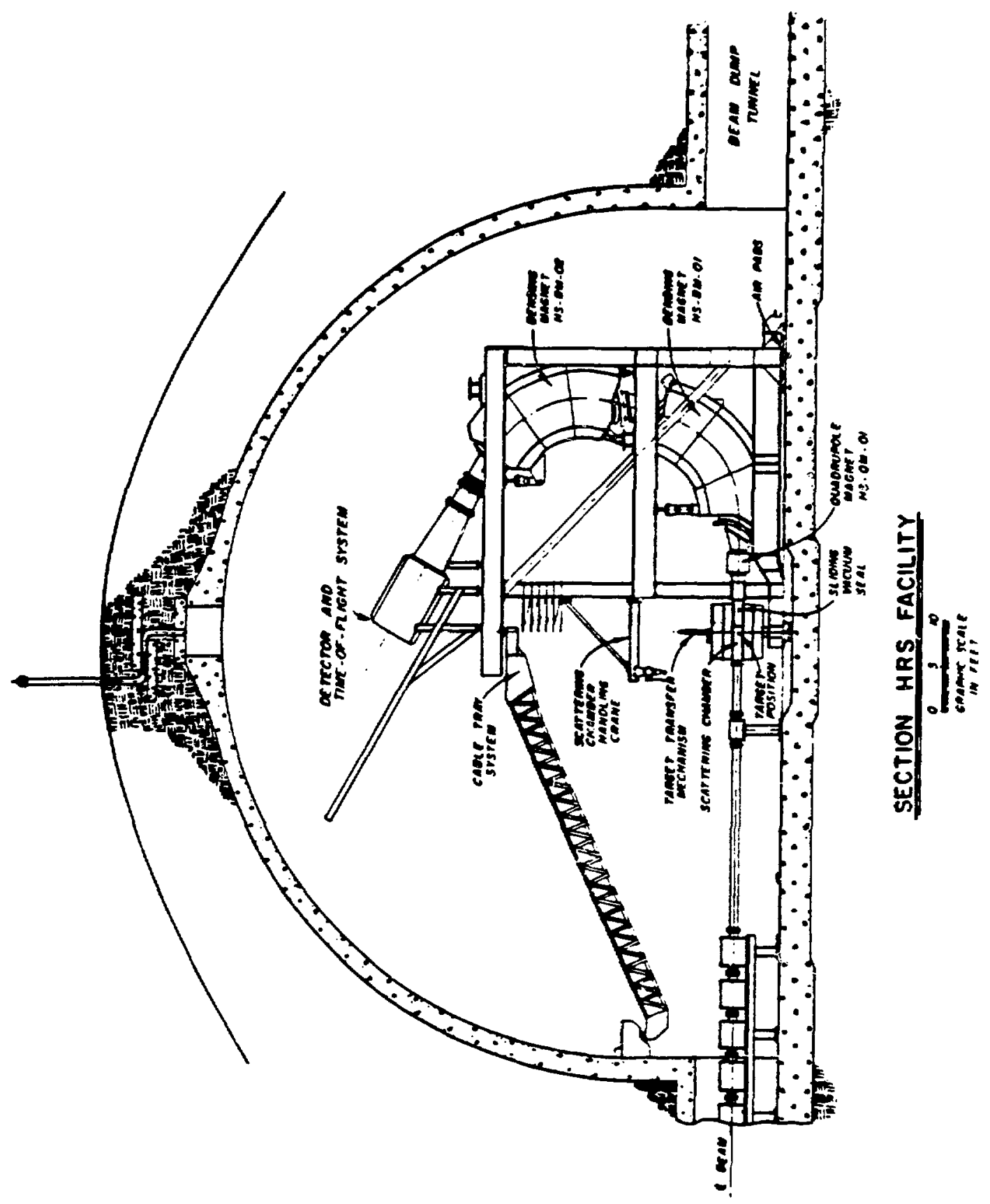

Fig. 12

The High Resolution Spectrometer (HRS). 
Table 4 - HRS Specifications.

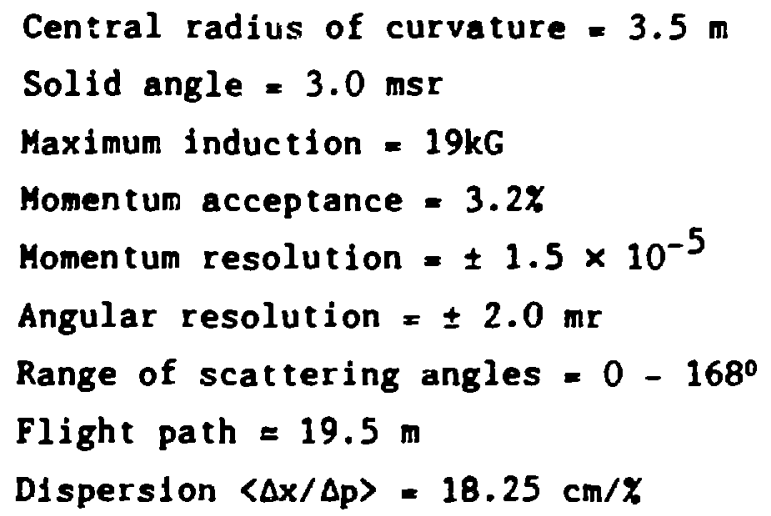

III-C. DETECTORS

Becsuse of the small angles involved in this experiment, the design and configuration of the detectors had to be planned carefully. At the smallest angle setting of the HRS for which data was obtained, $-0.83^{\circ}$, the elastically scattered protons as well as the unscattered beam were bent up onto the focal plane. Since passage of the intense beam through the focal plane detectors was unavoidable, a departure from the standard focal plane detection system used at the HRS had to be Implemented.

The detectors used in this experiment were of three types: scintillators, drift chambers, and ion chambers. These vere arranged differently on the focal plane, depending upon the angle setting of the HRS. For the data taken with the HRS at $-0.83^{\circ}$, the detectors were setup according to figlre 13. In this configuration, C1, C2, 


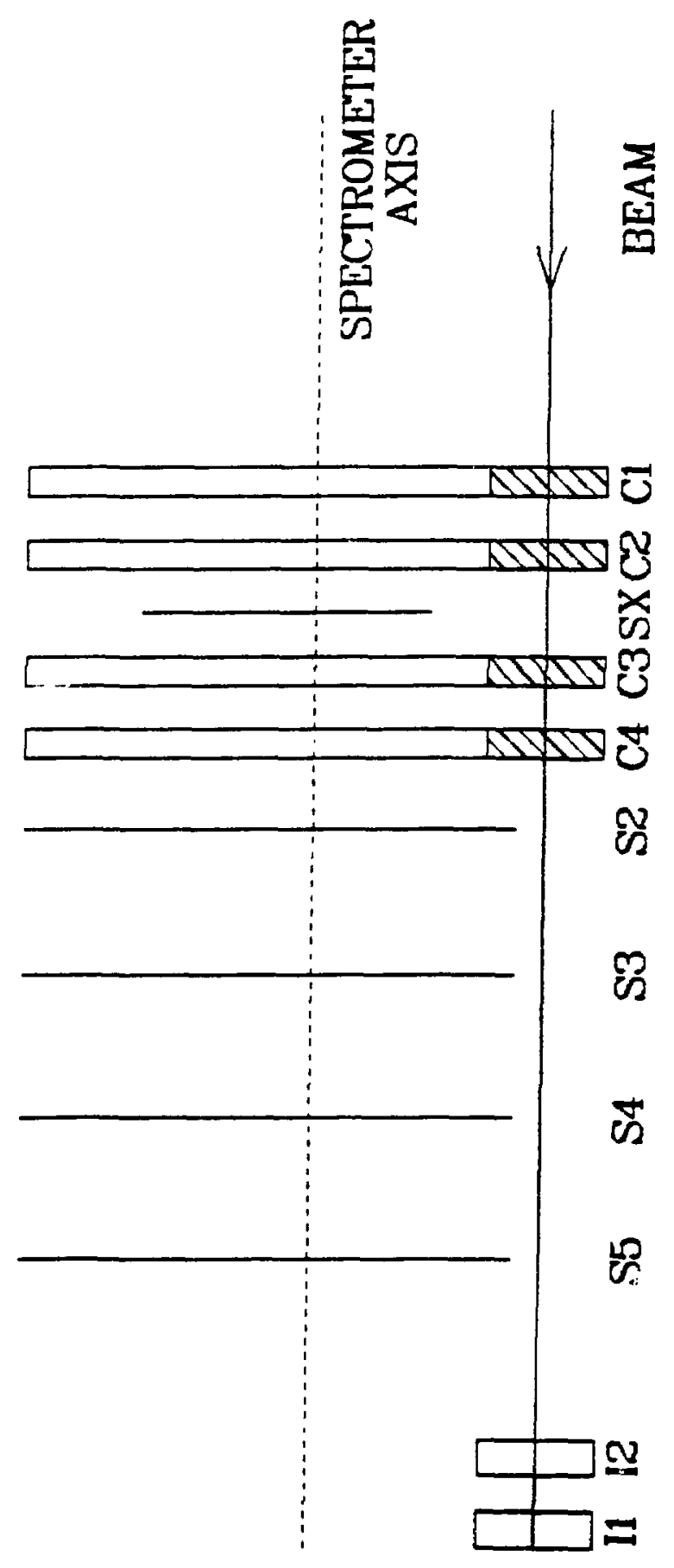

Fig. 13

Pocal plane detector arrangement (viewed fron above) for the case of bean passing through the detectors. 
C3, and $C 4$ are mult1w1re delay-line drift chambers, sX, s2, s3, s4, and S5 are plastic scintillators, and II and I2 are ion chambers. The drift chambers are of the delay-line type and were modified by putting dead regions in them for the beam to pass through. This was necessary in order to keep pulses in the chambers that would result from the beam going through them from overwhelming the scatcered protons of interest as well as to prevent damage to the chambers. The scintillator $\mathrm{SX}$ is $4^{\prime \prime} \times 4^{\prime \prime}$ while the remaining scintillators are $10 \mathrm{~cm} \times 56 \mathrm{~cm}$ with photomultiplier tubes at both ends. The ion chambers I1 and I2 were used to measure the intensity of the beam.

The plastic scintillators vere used in coincidence to form a trigger, a signal signifying passage of a particle through the detector system. With the beam projected on the focal plane, the coincidence used was

$$
\text { EVENT }=\$ \mathrm{SX} \cdot \$ 2 \cdot \$ 3 \cdot \$ 5
$$

The small sX counter was inserted between $\mathrm{C2}$ and $\mathrm{C3}$ in order to decrease accidental event triggers that would result from the coincidence S2 - S3 - S5. The source of these accidental event triggers is easily seen in figure 13. Scintillators $\$ 2,53$, and 55 used in the trigger were positioned as close to the beam as possible using a string to represent the path of the beam. Thus these scintillators overlapped the dead regions of the chambers. Particles could pass through the dead regions of the chambers and then proceed to trigger the coincidence $\mathrm{S2} \cdot \mathrm{S3} \cdot \mathrm{S} 5$. Placing $\mathrm{SX}$ between $\mathrm{C2}$ and 
C3 as shown eliminated this problem. Having SX in the trigger ensured that only particles passing through the active areas of the chambers could create a trigger. SX therefore determined the angular acceptance of the focal plane detector system.

For HRS angle settings where the beam was not bent onto the focal plane, SX was removed and the other scintillators vere positioned to include the full acceptance of the focal plane (1e., to include the region where the beam would otherwise pass through in figure 13). An event in this configuration was then specified by the colncidence

$$
\text { EVENT }=\mathrm{S} 2 \cdot \mathrm{S3} \cdot \mathrm{S4} \cdot \mathrm{S} 5
$$

Pulse height and time-of-flight information from these scintillators was used for particle identification. Nearly all particles detected were found to be elastically scattered protons.

The ion chambers mounted behind the focal plane detectors were each filled with different gases. One, denoted ERO2 in this experiment, was filled with $-60 \%$ xenon and $-40 \%$ ethane. The other, denoted ER04, was filled with $-60 \%$ krypton and $-40 \%$ ethane. Both were maintained at $600 \mathrm{~mm} \mathrm{Hg}$. Large dark currents were observed when the beam was off, and this had to be dealt with accordingly. At HRS angles where the beam was not on the focal plane, these ion chambers were placed downstream of the target in the scattering chamber. As larger beam intensities were permitted at these angles, BRO2 and ER04 were filled to $49 \mathrm{~mm} \mathrm{Hg}$ and $200 \mathrm{~mm} \mathrm{Hg}$ respectively. 
Each of the four delay line drift chambers used for position information consisted of a stack of planes as shown in figure 14 . These planes were screwed together around the edges to form a chamber providing both $x$ and $y$ position information. The $x$ and $y$ planes vere composed of anode wires connected to a $2.5 \mathrm{~ns} / \mathrm{cm}$ delay iine and cathode wires in between each anode wire that were alternately bussed together. The anode wires were spaced $8 \mathrm{~mm}$ apart and maintained at a voltage of $\sim 1950 \mathrm{~V}$, while the cathode wires were grounded. On both sides of the wire planes were cathode planes, also connected to ground. These cathode planes were of two types. In between the $x$ and $y$ wire planes was an aluminized mylar plane $-5 \mu \mathrm{m}$ thick. On the opposite sides of the $x$ and $y$ planes were ground planes made of

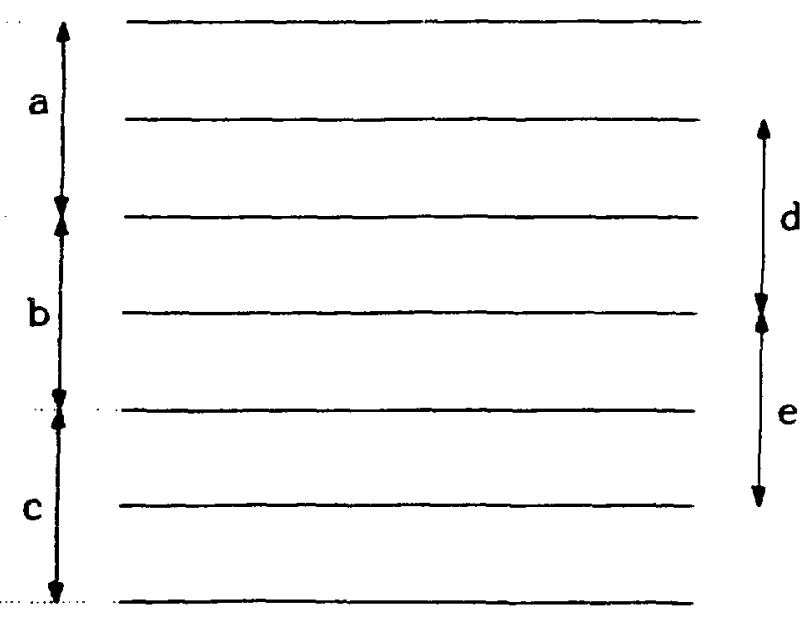

CHAMBER EDCE

GROLIND WIRE PLANE

X PLANE

ALUMINIZED MYLAR GROLND PLANE

Y PLANE

GROUND WIRE PLANE

CHAMBER EDGE

Fig. 14

Side view of chamber showing each of the constituent planes $(a=c=9 \mathrm{~mm}, b=d=e=9.5 \mathrm{~mm})$. 
copper clad aluminum wires spaced $2 \mathrm{~mm}$ apart and perpendicular to the direction of the wires in the $x$ and $y$ planes. All these planes were sandwiched together in between planes with mylar vindows that make the edge of the chamber. A gas mixture of $65 \%$ argon, $35 \%$ isobutane, and 0.107 freon flowed through this system of planes.

As the drift chambers required nodification, a considerable amount of time was spent preparing them to be used in this experiment. A method had to be developed to make charge collection near a segment of an anode vire non-existent, or at least minimal. After trying several different possible ways of accomplishing this goal, the most promising candidate for success was developed, tested, and finally employed in the experiment itself. Figure 15 illustrates the method used to create a region of minimal charge collection around an anode wire. The idea is to enclose the wire in a good insulating material to prevent negative charges from reaching the positively charged anode wire. Then, as negative charge builds up on the surface of the insulator, the electric field locally around the anode wre (1e., outside the insulator) decreases, further contributing to low charge collection on the anode.

The concept is simple in theory, but difficult in practice. Getting a good enough insulator to uniformly cover an anode wire is no easy rask. The insulator used to cover the anode wire was chosen to be an epoxy resin. When this epoxy resin was applied to the bare wire, it beaded to the point that there were places between the beads where no epoxy covered the wire. Successive application of more coats of epoxy only made the beads larger, without filling the space 


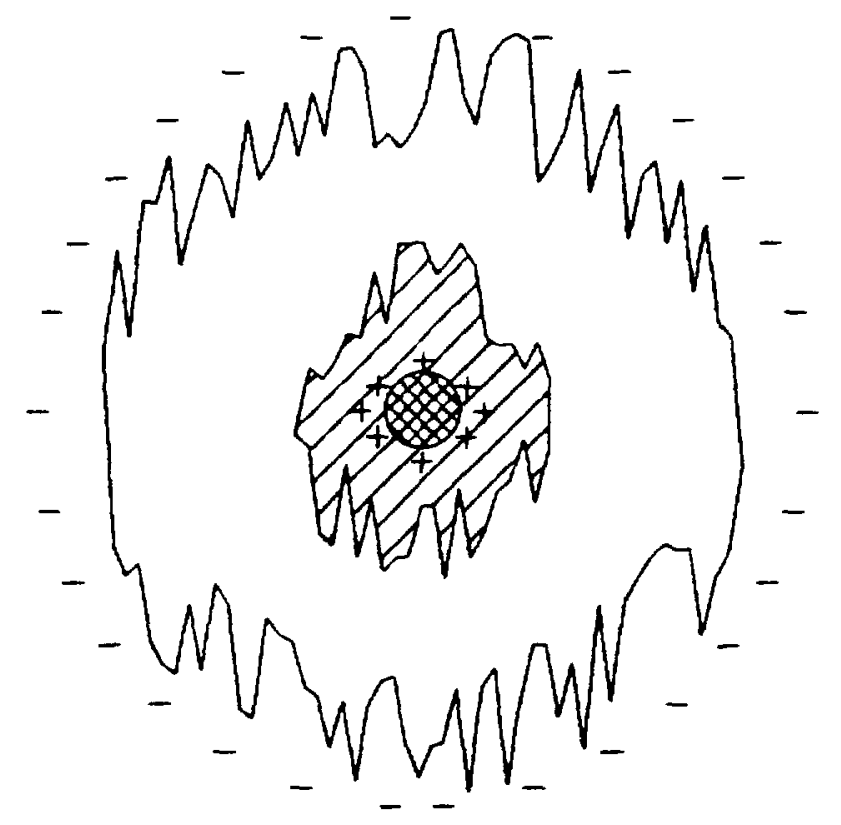

WIRE

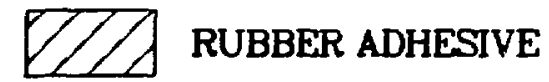

EPOXY

Fig. 15

Cross sectional view of an epoxied anode wire.

between them. Since the wire was made of gold-plated tungsten, the problem was particular?y severe, as this substance is notorious for creating this type of problem. As a means of getting around this difficulty, a coat of rubber adhesive mixed with trichloro-ethylene was first applied to the wire in order to give the epoxy a better surface to stick to. Then the epoxy was mixed with cab-o-sil, a thickening agent used to give the epoxy a false viscosity. The epoxy could then be easiiy coated onto the rubber adhesive without beading.

Various tests were made to determine how well this method works. Using cosmic rays, a test beam consisting of nostly pions, and then 
finelly the proton bean at the HRS, the deadness of the epoxied vires vas investigated. In figure 16, a view of a chaber uniformly Illuninated vith cosalc rays is shorn. The vires in only one plane have been coated vith epoxy, and vithout rubber adhesive. Figure 17 shows the vires in two different reglons of the chamber in figure 16. Clearly the region where epoxy has ben applied is very inefficient for charge collection. It should be noted that this is for the vorst case, 1e. the case where the epoxy beaded badly. If the vires had been coated first vith rubber adhesive and $1 f$ both planes had been

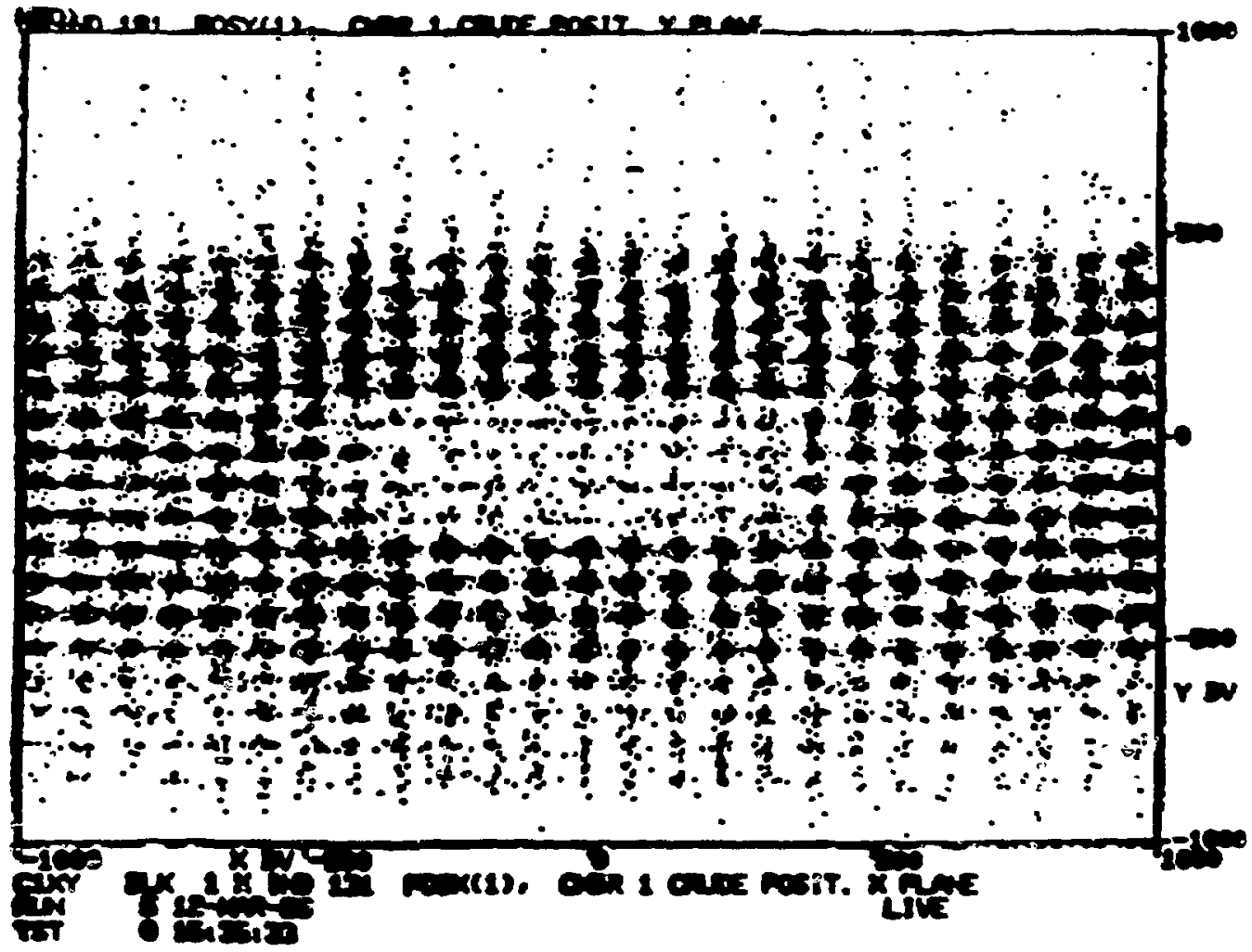

Fis. 16

Dotplot of $X$ Vs. I anode vire positions in a chamber In which the vires of one plane have been coated vith epoxy in the center of the chanber. 

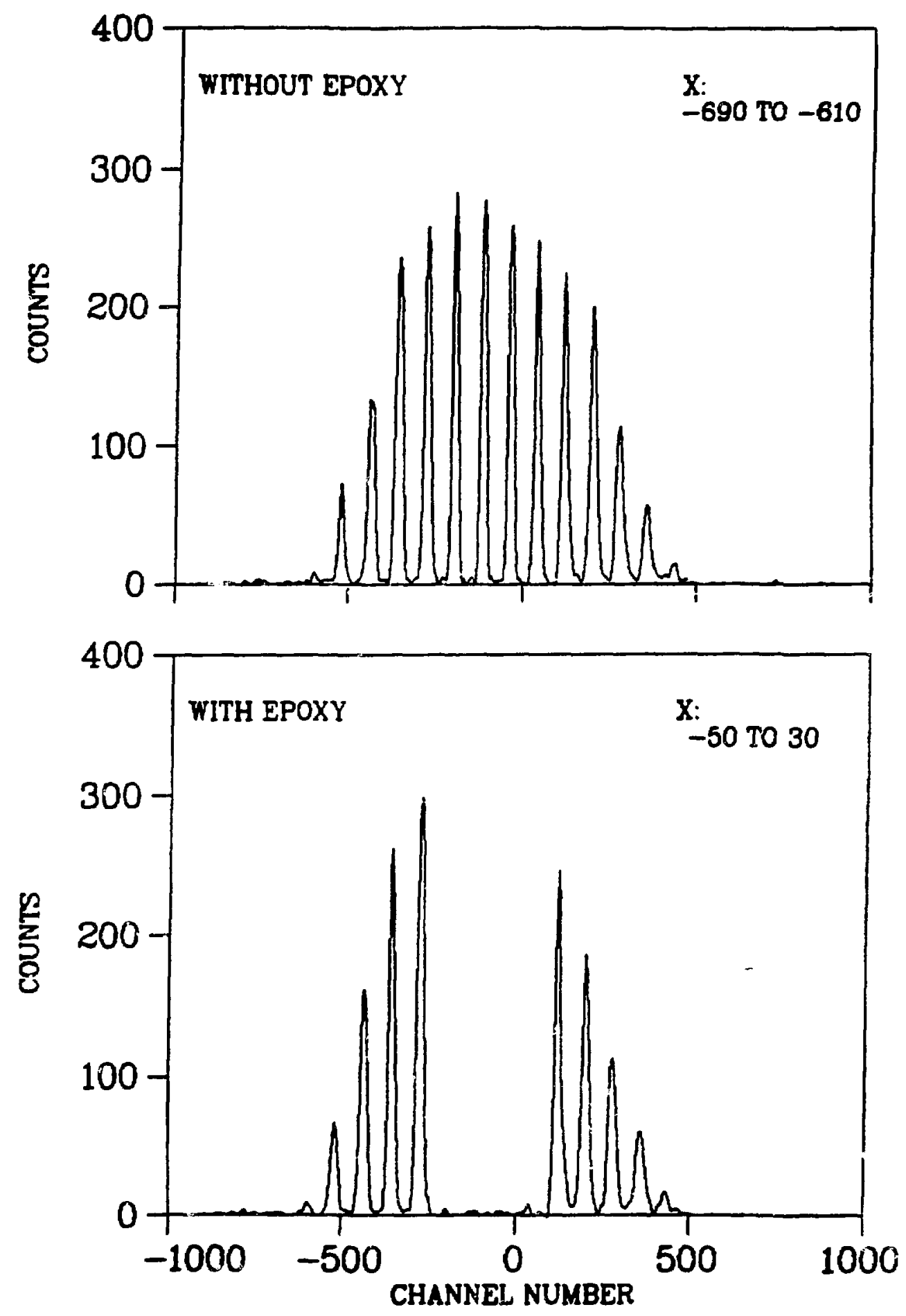

F1g. 17

Histogran of vites in the $Y$ plane in the reston vithout epoxy (top); $T$ uires in the epoxied region (botton). 
epoxied, the histogram of figure 17 would look even better. Indeed, when a chamber with epoxied wires in both planes was tested at the HRS with a pencil beam hitting the center of a dead region 3 wires $x$ 6 wires in area, only 3,642 events out of 108,699 average beam particles/sec were detected in the dead region, indicating that the dead region was about $97 \%$ dead.

A scale diagran of a chamber with a dead region as it was used to obtain the data in this work is shown in figure 18. The dead region covered 5 wires horizontally and 7 wires vertically and was piaced so that the elastic spectral line would fit comfortably next to the dead region. With a trigger rate of about 25 events/sec these chambers drew $-1-2 \mu \mathrm{A}$ of current with a combined chamber efficiency (all chambers giving good positions) of - 55\%. At a trigger rate of - $10 / \mathrm{sec}$ the chamber efficiency was - $70 \%$ with minimal current being drawn. At a trigger rate of $\sim 70 / \mathrm{sec}$ the chamber efficiency was $\sim 30 \%$ with the chambers drawing $\geq 2.5 \mu \mathrm{A}$. As the number of good events writen to tape per second is equal to the product of the trigger rate times the chamber efficiency, clearly the best rate to take data is $\sim 70 / \mathrm{sec}$. However, chambers should not draw more than about $-2 \mu \mathrm{A}$ if they are to last long. Therefore, a rate of $25 / \mathrm{sec}$ was the best rate to take data. At angles where the beam did not pass through the chambers, taping speed was the limiting factor determining the rate at which data could be taken. A trigger rate of $-500 / \mathrm{sec}$ with a chamber efficlency of - $92 \%$ yas the case for these angles. Current drawn was $<2 \mu \mathrm{A}$.

In addition to the problem of dealing with the beam passing 


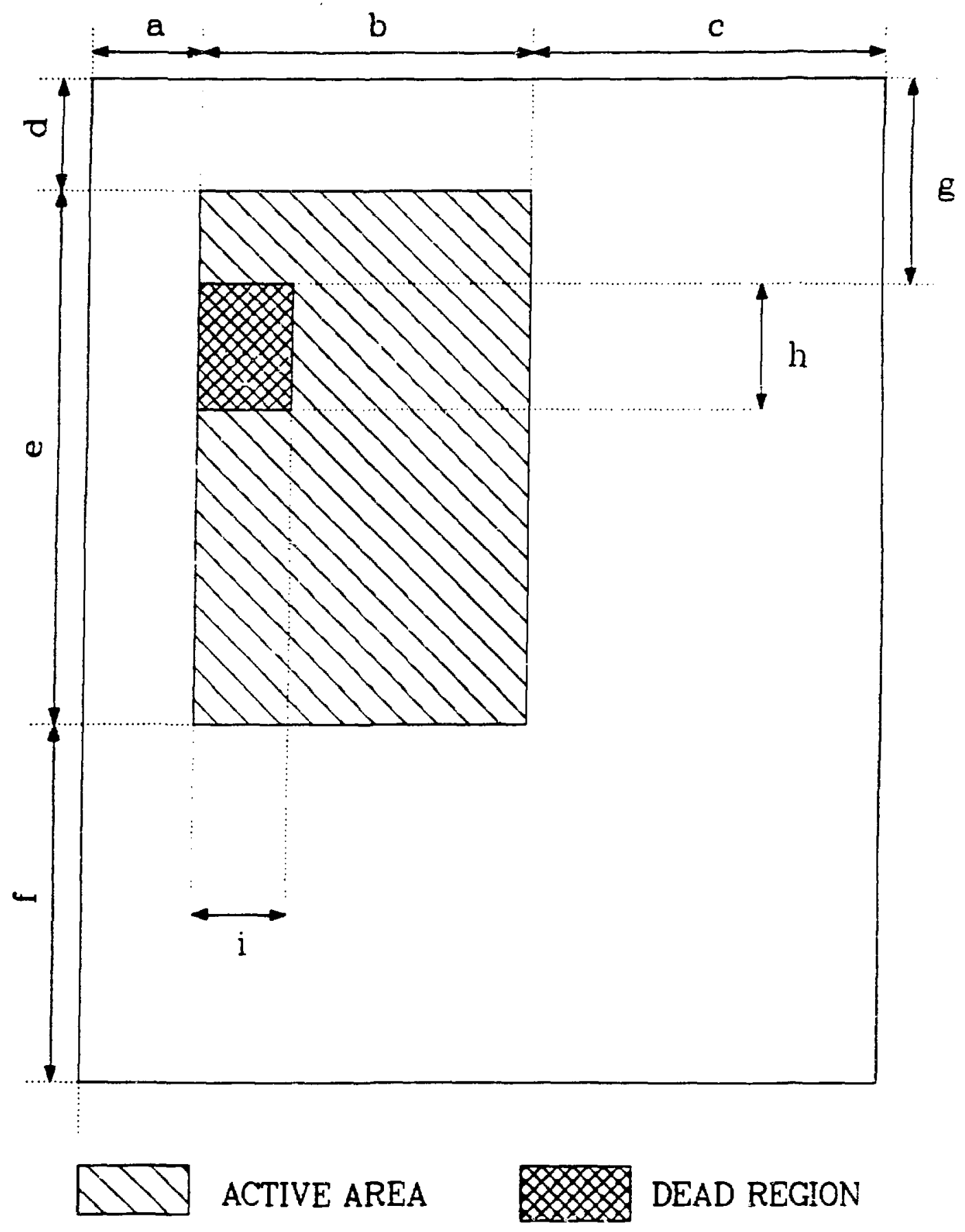

Fig. 18

Delay line drift chamber used in this experiment

$(a=5.0 \mathrm{~cm}, b=15.0 \mathrm{~cm}, c=16.0 \mathrm{~cm}, d=5.0 \mathrm{~cm}, e=24.0 \mathrm{~cm}$, $\tilde{\mathrm{r}}=16.0 \mathrm{~cm}, \mathrm{~g}=9.2 \mathrm{~cm}, \mathrm{~h}=5.72 \mathrm{~cm}, \quad \mathrm{i}=4.21 \mathrm{~cm})$. 
through the drift chambers, the left-right ambigulty had to be resolved. This is the problem of determining the side (left or right) of the anode wire through which the ionizing particle passed. This information is needed so that the drift distance obtained from the drift time can be used to correct the position given by the anode wire.

Particle Position = Wire position \pm Drift Distance (155)

Resolving the left-right ambiguity is therefore determining the choice of using the plus or minus sign in eqn. (155). We discuss here two methods commonly used to resolve the left-right ambiguity. The first method, using induced cathode pulses, was tested to determine its feasibility in this experiment. This method was abandoned, for reasons discussed below, in favor of the method of offset chamber planes.

The method of induced cathode pulses makes use of the induced positive pulses on the cathode wires which alternate with the anode wires. These cathode wires are alternately bussed together. One set of bussed cathode wires is called the odd wire set and the other bussed set makes up the even wire set. This is shown in figure 19 . For all particles passing through the chamber, positive pulses are indured on both the odd wire and the even wire on the two sides of the anode wire picking up the negative signal. For example, in the event depicted in figure 19 by an $x$, anode wire $\$ 1$ fires and positive charge is inciuced on the nearest odd and even wires. Since the event 


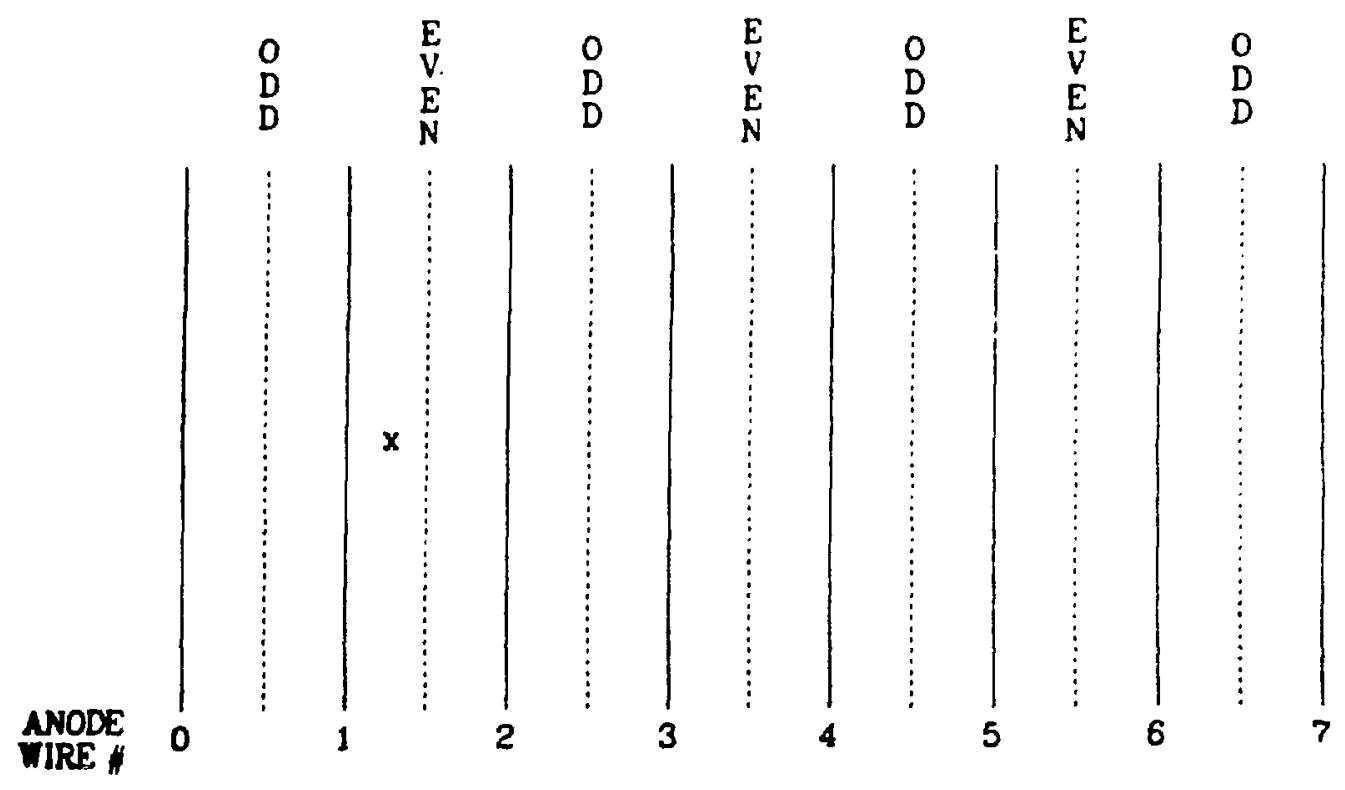

Fig. 19

Location of odd and even cathode wires.

is on the even side of the anode wire, more charge will be pickes up on the even wire than on the odd wire. Thus, by comparing the pulse height of the signals from the odd and even lines, the left-right ambiguity can be resolved. Note that if the event in figure 19 vere just to the right of anode vire $\$ 2$ instead of $\$ 1$, then the pulse height of the pulse coming out of the odd line would be greater than that of the pulse coming out of the even line. This leads to the following form for the position of the event:

$$
\text { Pos }=n S+D(-1)^{n+m}
$$


where Pos is the position of the event, $n$ is the number of the anode wire that fires, $S$ is the spacing between anode vires, and $D$ is the drift distance. $m$ is given by

$$
\begin{array}{rlrl}
m & =1 & & \text { EVEN > ODD } \\
& =0 & \text { EVEN < ODD }
\end{array}
$$

where $O D D$ and EVEN are the pulse heights coming from the odd and even lines respectively.

To completely determine the position of the event, one must therefore determine whether $m=1$ or 0 in eqn.(156), ie. vhich of the signals coming from the two bussed lines has the greater pulse helght. To do this, the signals from the odd and even lines are each amplified and put into ADC's. Figure 20 shows a typical ADC spectrum from one of these lines. The small ard large peaks correspond to low and high gain mode events respectively. By increasing the voltage on the chamber, one can make all the events high galn events, so that the small peak disappears. It is desirable to do this, as higher amplitude pulses are easier to deal with when one tries to determine which cathode signal (odd or even) has the larger pulse height. This is easily seen in figure 21, where a dot-plot of the ADC's for the even line vs. the odd line is displayed. This plot is gated on events associated with a single anode vire for reasons discussed below. Clearly, the left-right ambiguity becones easier to resolve with larger pulse sizes as the difference in pulse heights, Odd-Minus-Even (OKE), becomes larger. 


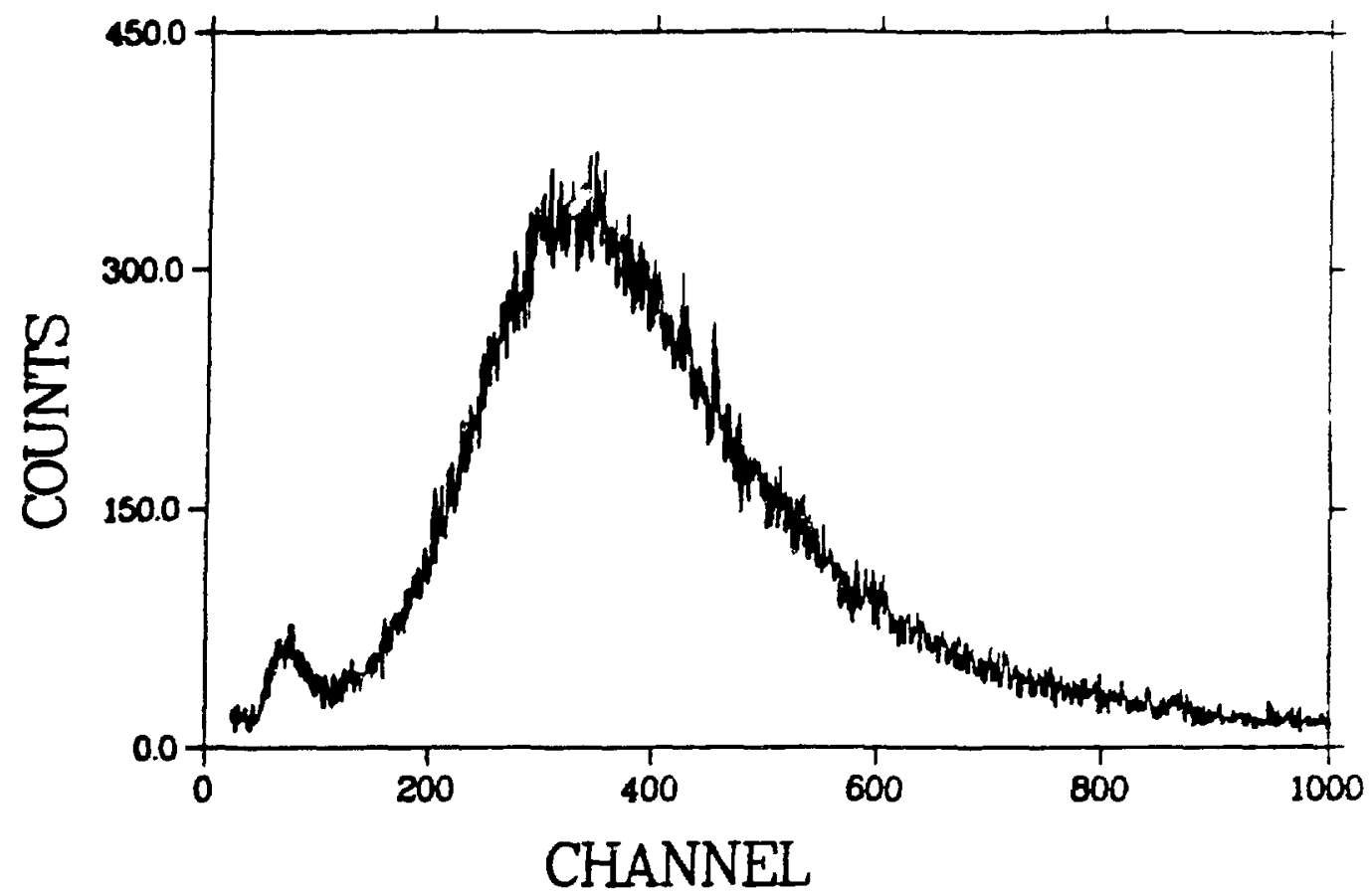

Fig. 20

ADC apectra of signals coming from cathode uires.

One of the problems in using odd-even signals is that the centrold of OrE can be anode vire dependent. An example of this is shown in figure 22, where a plot of OHE vs. position in the chamber reveals that one cannot siaply take the difference in the ADC values of the odd and even lines and resolve left from right. The "holes" in each vertical band in figure 22 need to be aligned. A connon vay of doing this is to make all the odd and even ADC spectra for Individual anode vires have the same position and vidth. Thus before calculating OuE, the pulse height for cathode pulses is adjusted eccording to 


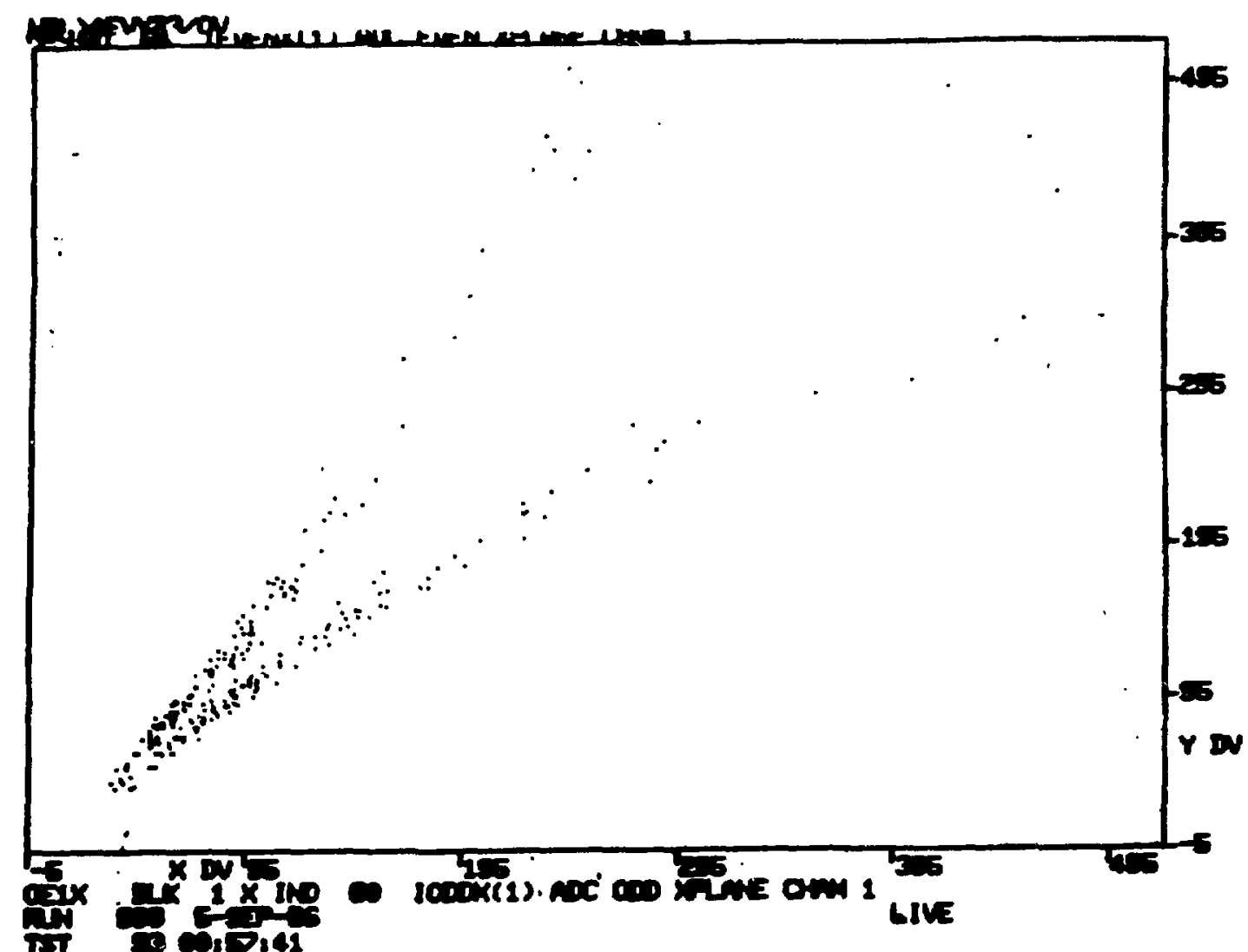

Fig. 21

Dot-plot of odd versus even ADC's.

$A O D D=(O D D-\operatorname{OFFSET}(n)) * \operatorname{GAIN}(n)$

where AODD is the adjusted pulse height for the odd line, OFFSET(n) is an offset that is different for each of the $n$ anode wires, and GAIN(n) is a mutiplication factor that is also anode vire dependent. A similar expression is used for the even line. OME then is defined as

OHE = AODD - AEVEN 


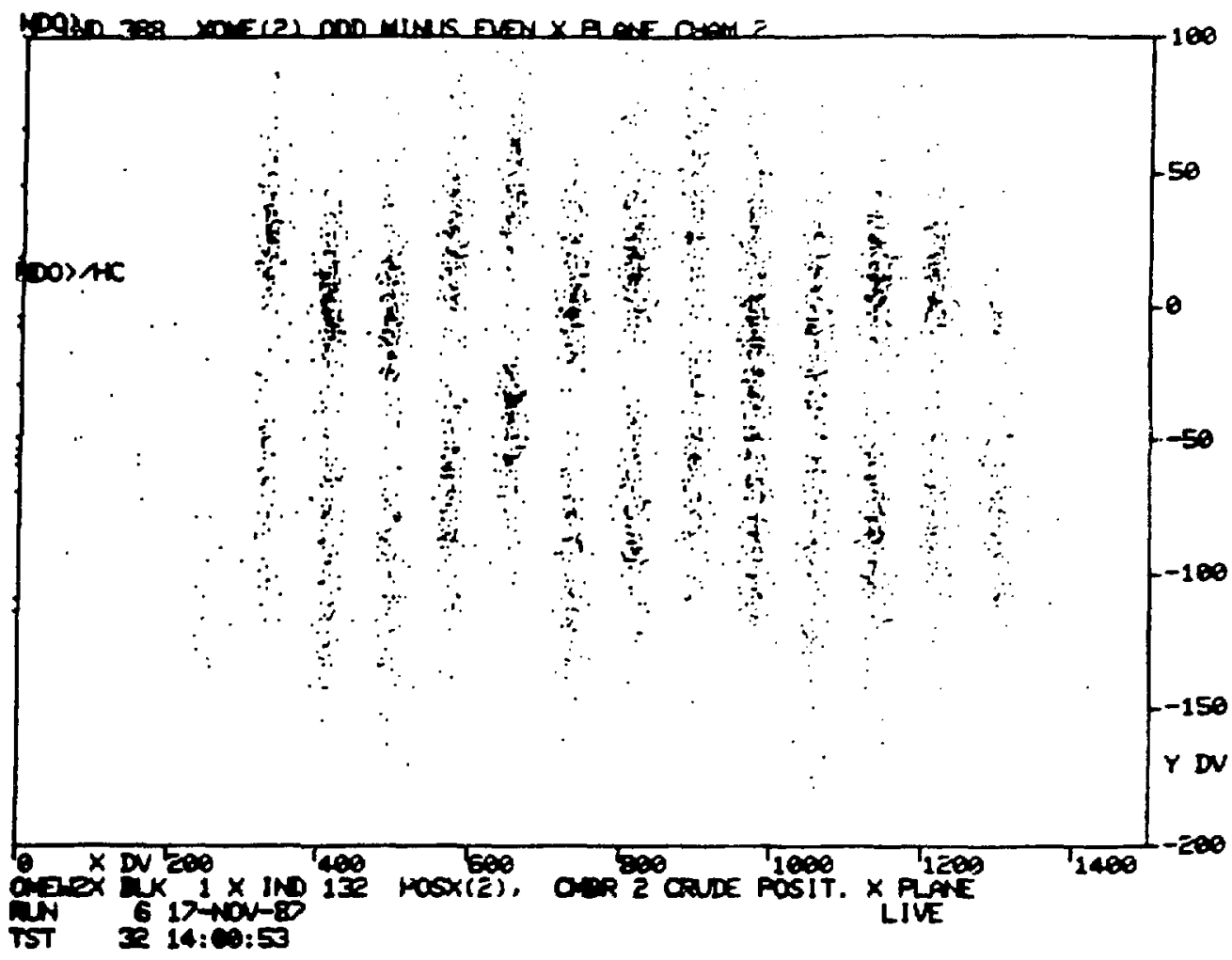

Fig. 22

Dot-plot of OME versus position in chamber.

Clearly this anode wire dependency of OHE complicates things. In this experiment, with $4 X$ planes of 30 wires each and $4 Y$ planes of 20 wires each, this requires $120+80=200$ offsets and 200 gains! But it is also clear that without this large number of offsets and gains, left could not be distinguished from right. In the top of flgure 23 is a histogram of OHE showing that with all anode wires Included and no offsets or gains adjusting the pulse height, the left peak nerges with the right, making the resolving of the left-right abigulty 1mpossible. In the bottom of the figure, only one anode 


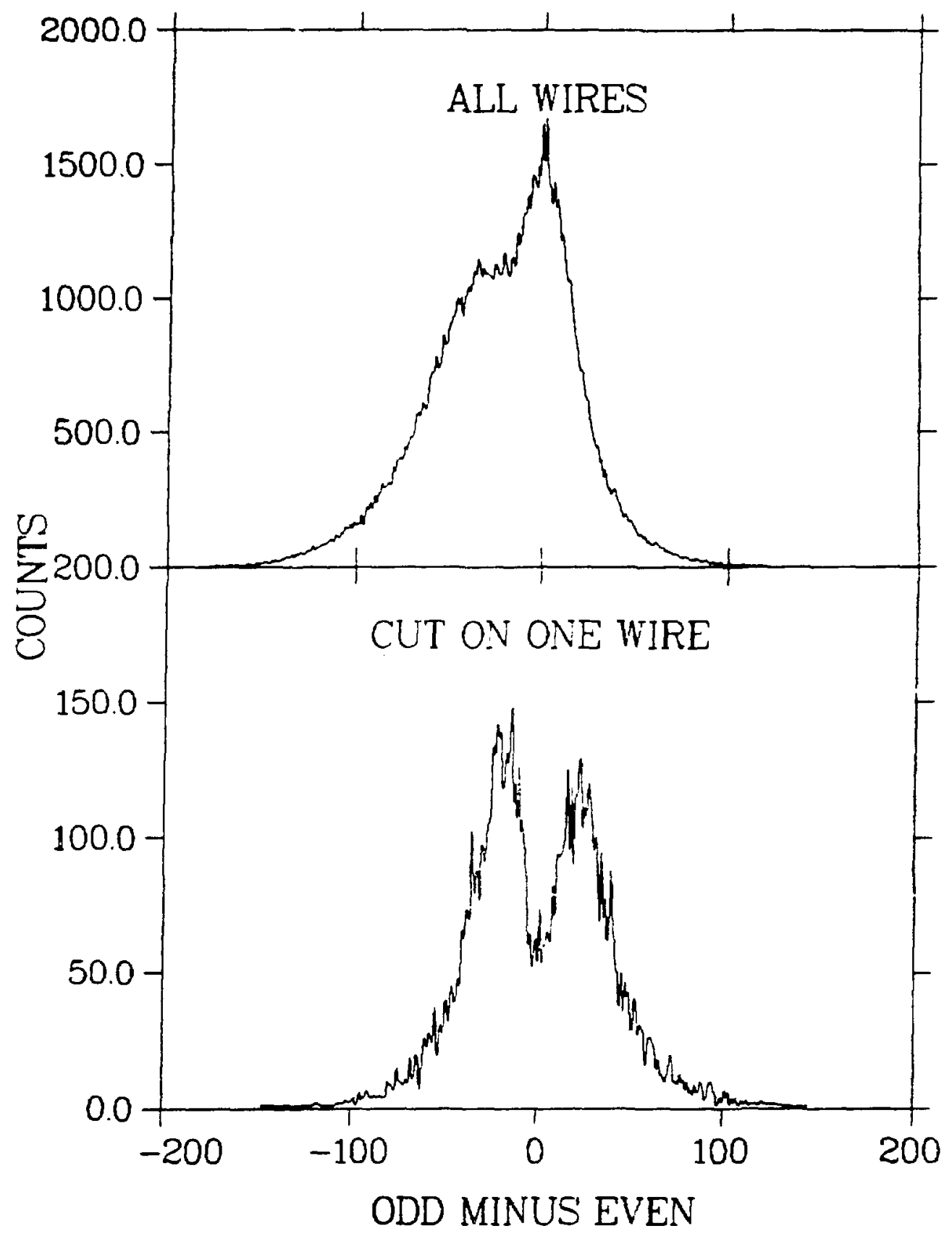

Fig. 23

Histogram of OME with events from all anode vires included (top), and with events from only one anode wire included (bot tom). 
wire is included, showing that there are actually two peaks corresponding to odd and even events.

Puting aside the complications resulting from the anode wire factor in separating the left and right peaks, the question of chamber efficiency arises. If a good chamber event is an event in which an odd and even signal are present and do not overflow the ADC, then maximum chamber efficiency dictates that there be as few events in the overflow bins of the ADC's as possible. There are four parameters that can be adjusted to control the position and shape of the pulse height spectra in the ADC's: 1) chamber voltage, 2) signal attenuation, 3) amplifier gain, and 4) the DC level of the amplifier. Tweaking any of these parameters causes the dots in figure 21 io move along the two curves, the concave up curve corresponding to even events and the concave down curve corresponding to odd events. The problem is that no adjustment of these parameters causes the two curves to move apart from each other. Thus for best left-right peak separation, the higher channel $A D C$ events are the most useful. Figure 24 shows the same histograms as in figure 23 except that only events in ADC channels greater than 250 (from a 512 channel ADC) are included. Left-right separation, especially for one anode wire only, is even better than in figure 23 .

If we want to resolve the left-right ambiguity well, we must have most of the events in the higher channels of the ADC. This can be done by adjusting the four parameters. However, when this is done, there are many events in the overflow bin of the ADC. This causes the chamber efficiency to go down. Thus we are stuck with the 


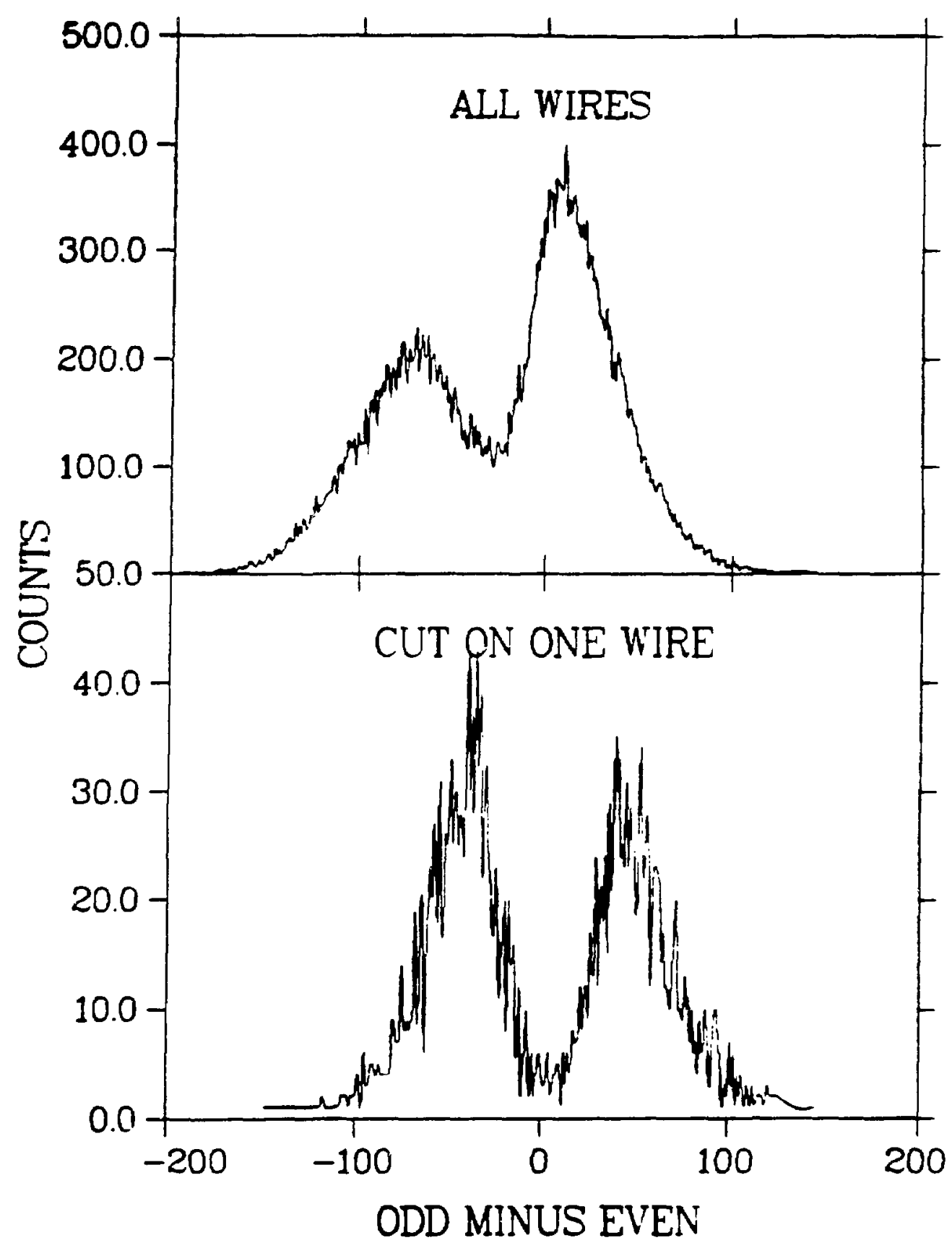

Fig. 24

Same as figure 23 except that only events in $A D C$ channels greater than 250 are included. 
following cholce: we can have good left-right peak separation and poor chamber efficlency, or good chamber efficiency and poor left-right peak separation. In fact, for the case where left-right peak separation is good, the observed chamber efficiency per plane was - 60\%. Since two $X$ and two $Y$ planes are the minimum number of planes required to define a track, the overall chamber efficiency would be only $(.6)^{4}=12.8 \%$. Perhaps this problem could have been remedied with an ADC having 1024 channels, but since no buffered 1024 channel ADC's were available at the time of the experiment, this method of resolving the left-right ambiguity was abandoned in favor of the method of offset planes.

The idea behind the method of offset planes is really quite simple. Two adjacent chambers are positioned such that the anode wires of one chamber overlap the cathode wires of the other. This is shown schematically in figure 25. In the figure, anode vire 11 of chamber 1 and anode wire $\$ 2$ of chamber 2 both fire. The left-right ambiguity then provides four possible track candidates, A, B, C, and D through the two chambers. To decide uhich track is the correct one, information from chambers 3 and 4 is used. If anode wire \#1 of chamber 3 and anode wire $\# 1$ of chamber 4 fire, then track $A$ is obviously the correct choice. If instead a higher wire number in each of chambers 3 and 4 fire, then track $A$ can be ruled out in tavor of one of the other three tracks. In this way, the left-right ambiguity is resolved for chambers 1 and 2. The process is then repeated in reverse to resolve the left-right ambiguity in chambers 3 and 4 . 

CHAMBER 4
(0) 1
(1)
(2)
(3)
CHAMBER 3
(0) 1
(1) 1
(2) 1

A B C D

CHAMBER 21 (0) 1 (1) (2)

CHAMBER 1 (0) (1): (2)

Fig. 25

The aethod of offset planes to resolve the left-right ambiguity. 


\section{III-D. ELECTRONICS AND DATA ACOUISITION}

As scattered particles passed through the system of detectors on the focal plane, the signals generated by the detectors were sent through long low loss cables to the counting house where they were processed. First, signals from the scintillators vere combinet to determine whether an event had occurred. Data was recorded for the scintillators and delay line chambers only if all the proper scintillator signals formed a valid colncidence, and hence an event. Thus backgrounds of various sorts were eliminated. Figure 26 shows the electronics used to generate an event. For data taken at $-0.83^{\circ}$ S4 was removed from the colncidence while at larger angles $S X$ was removed. The timing of an event in both cases was determined by $\mathbf{s}$. All scintillator signals, except those coming into the feed through discriminators and going to the ADC's (Analog to Digltal Converters), are NIM standard signals. The width of the signals going into the meantimers and into the coincidences 52.53 and $54 \cdot 55$ are 10 ns. The Input and output signals to EVENT are 20ns.

Signals from the drift chambers were first translated to NIM in the experimental area before being relayed to the counting house. Figure 27 schematically lllustrates the handling of the small ( $3 \mathrm{mV}$ ) chamber signals. The raw chamber signals are anplified by two $\times 10$ anplifiers to bring the size of the pulses to a few hundred aillivolts. Then the signals are passed through apacitor to filter out low frequency noise and remove any de level at that point. In order for the tining of the signals to be independent of the pulse 


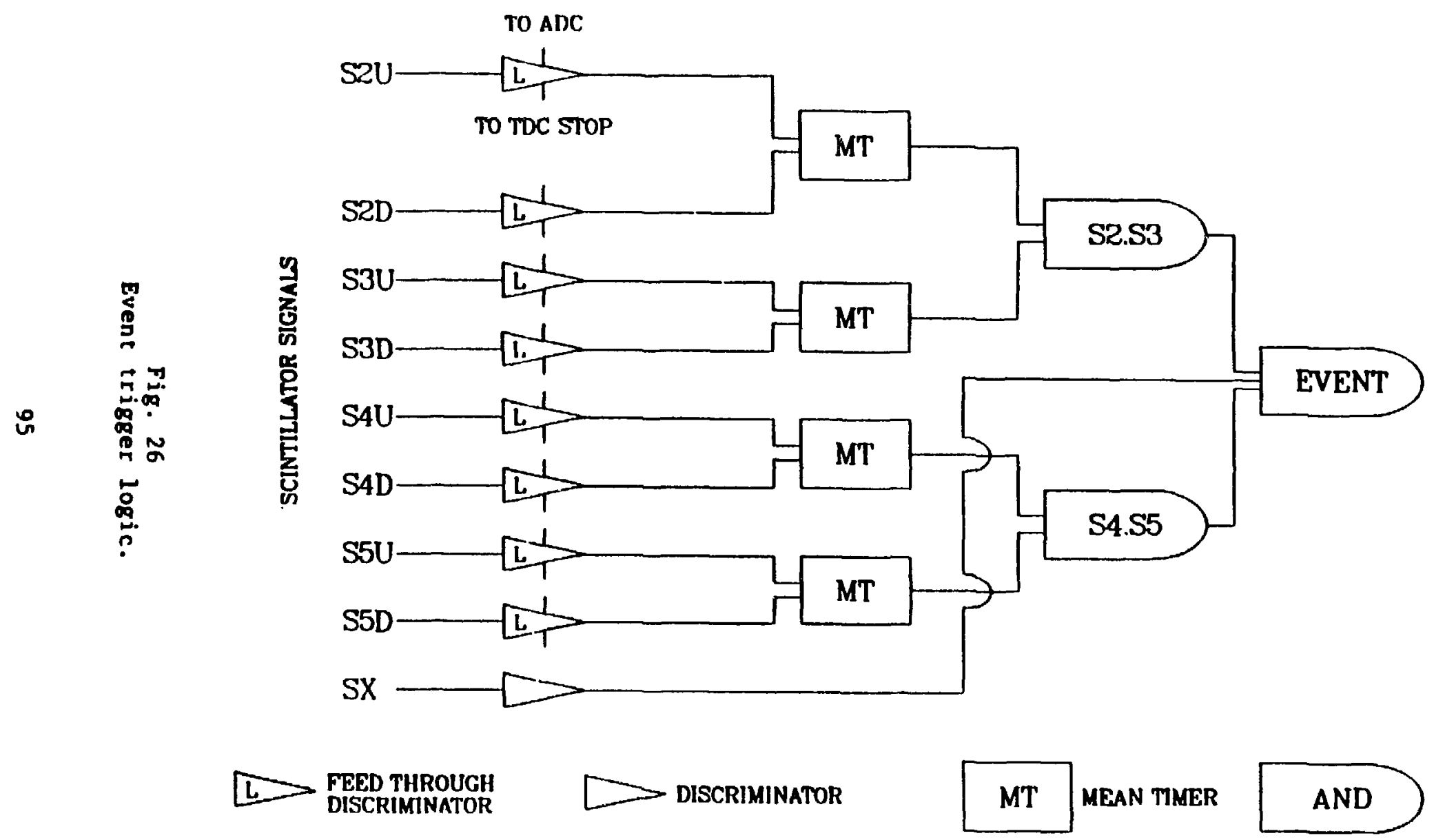




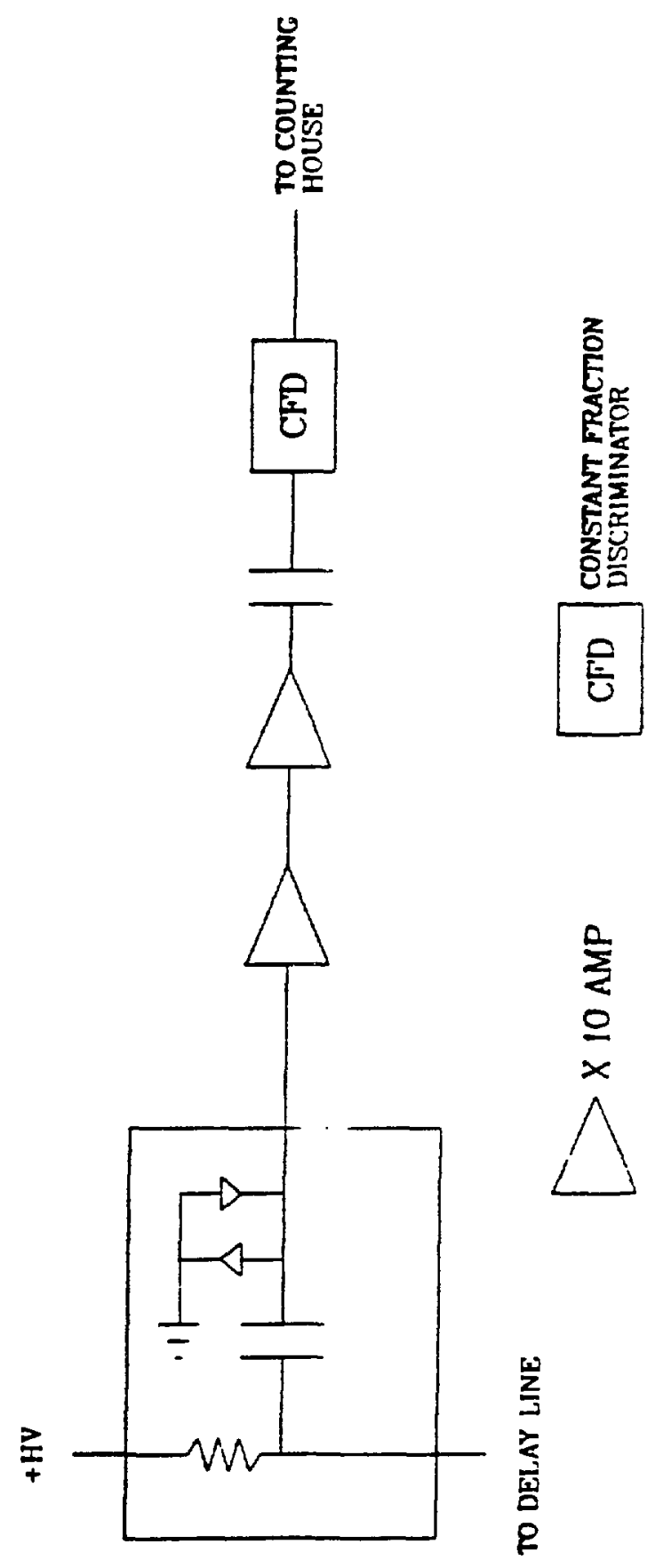

Fig. 27

The electronics used to handle chamber signals. 
height, the signals go through a constant fraction discriminator (CFD) and are translated into NIM. These signals then 80 into the counting house where their timing information is used to find the trajectories of the particles passing through the chambers.

Under certain conditions, a valid event triggers the storage of Information into ADC's and TOC's (Time to Digltal Converters). These ADC's and TDC's along with some scalers are part of the CAMAC crate systea used for the orderly transfer of raw data into a data stream. The ADC's and TDC's are FIFO (First In First Out) buffered, allowing up to 32 events to be stored in them. Whenever a valid event takes place and the ADC's and TDC's are not converting the previous event (CAMAC busy) and their buffers are not full, scintillator and chamber Information is stored in them. Since conversion time is of the order of $10 \mathrm{\mu s}$ and $1 \mathrm{t}$ takes about $300 \mathrm{\mu s}$ to read out an event (all TDC's and $A D C^{\prime}(s)$, data can be taken faster than 1 it is read out. This is highly advantageous since then several events can be taken during the macropulse of $-500 \mu s$ and then read out between beam bursts $(-10.2 m s)$.

A schematic diagram showing the triggering of the CAMAC modules is shown in figure 28. In addition to the trigger requiring a valid event and CAMAC not busy, a rungate must also be present. This rungate is a conglomeration of signals indicating that running conditions are present. These conditions are that the beam is on, that it is polarized, a run is in progress, the magnets are on, etc. When all these requirements are met, triggers can be sent to the various $A D C$ 's, TDC's, scalers, and vetoes. 


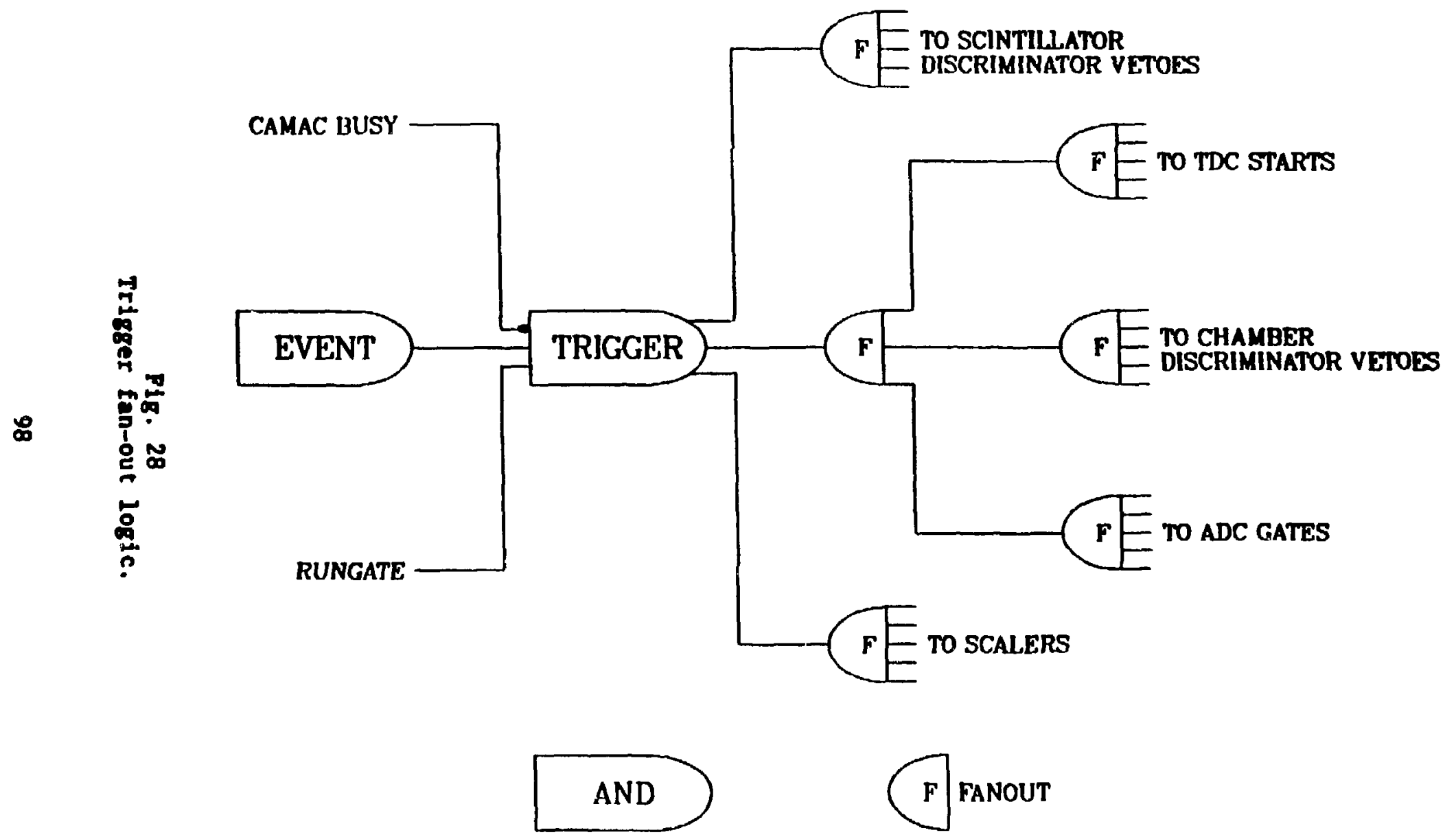


In addition to the trigger signals sent to the TDC's and $A D C$ 's, another trigger is sent to device called the Event Trigger Module (ETM) which causes all the ADC's and TDC's to be read and cleared. Th1s trigger is vetoed by the busy from the ETM so thet an event can only be read out after the previous event has been read out. It is because the length of the ETM busy signal is much longer than the CAMAC busy signal that buffered electronics is so useful. Events can be acquited even then a previous event is being read out.

It is useful to consider the flow of events through the electronics from a time perspective in order to better understand how buffered electronics work. Let us suppose that the event rate is high, say 1 event per us. When a run starts, the first event from the first macropulse creates a trigger which causes $A D C$ and $T D C$ information to be stored for this event. While this is taking place, the system is vetoed from taking any more events for the duration of the conversion time of the TDC's and ADC's (-10us). This means that the next 10 events will not go through the system. Thus every 10us an event is placed into the buffers of the ADC's and TDC's unt1l those buffers become full. Meanwhile, events are read out of the buffered CaMaC modules on a first-in-first-out basis. The first event that came into the electronics at the start of the run is read out as soon as the buffered modules have finished converting that event from analog to digital form. While this event is being read out, the ETM is vetoed from reading the next event in the buffered modules for the duration of the time it takes to read an event ( - 300us). Thus at most two events can be read from the buffered 
modules in a 500us macropulse, but as many as 34 events ( 32 filling the buffers +2 read out) can be accepted by the modules. So

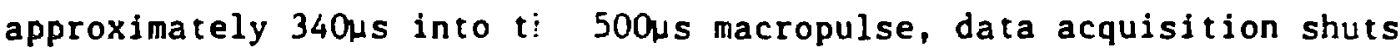
down until there is room in the buffered modules for another event to be stored.

An event rate of 1 per us clearly fills the buffers as fast as they can be filled and allows only data from the first part of the macropulse to be stored. To obtain events from the entire macropulse one would need an event rate lower than 34 events $/ 500 \mu s=68$ per ms. Once the buffers have been filled and the time between macropulses starts, the buffers are read out at the rate of 1 event every $\sim 300 \mathrm{ws}$. Since the time between macropulses is $-10 \mathrm{~ms}$, the buffers are completely empty by the time the next 500us macropulse is sent down the accelerator. The event storage process then repeats itself with each successive macropulse time-between-macropulses sequence.

The advantage of buffered electronics is quite apparent. Non-buffered electronics is the equivalent of buffered electronics in which the buffered modules only have room for one event in their buffers instead of 32 . One can therefore repeat the above discussion for 1 event buffered modules to find that the maximum number of events that one can take during a 500us macropulse would be 3 instead of 34 ( 1 filling the buffers and 2 read out).

The CAMAC crate system interfaces to the data acquisition computer by means of an MBD (Micro-programmable Branch Driver). This is shown schematically in figure 29. Only one of several CAMAC crates is shown. Whenever the ETM is triggered, the MBD reads all 

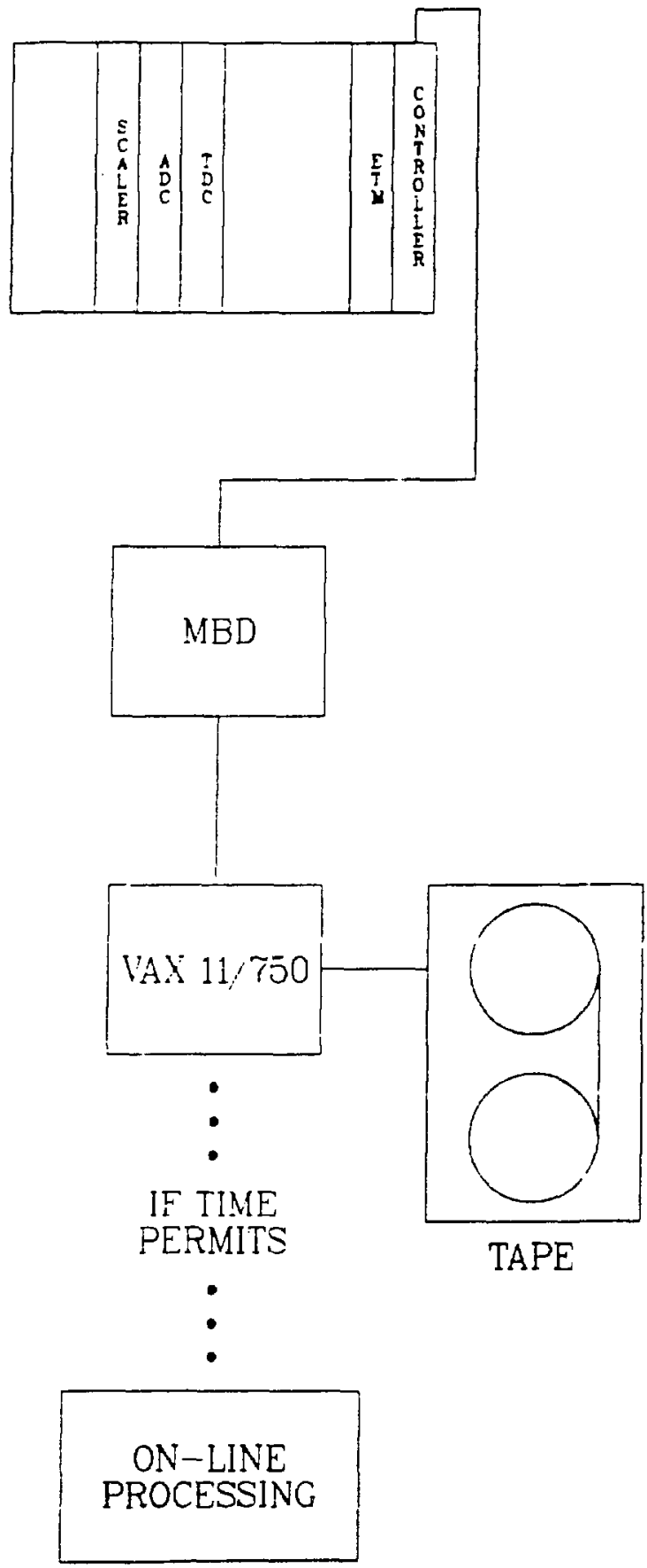

Fig. 29

Data acquisition system.

Only one of several CAMAC crates is shown. 
the data required for the event. There are basically two kinds cf events: a normal particle event triggered by the scintillator colncidences, and a scaler event triggered by a clock. For normal particle events the MBD reads $A D C^{\prime}$ s and $T D C^{\prime} s$, whereas for scaler events the MBD reads only scalers. When the MBD is full (1500 word buffer) it dumps the data into the buffers of the VaX which writes the data to tape. If the computer has time, le. it is not busy receiving data or writing it to tape, it allows the data acquisition program to process the event on-line. For data taken with the beam on the focal plane, the event rate was low enough that nearly all events could be processed on-line. At the larger angles, the event rate was much higher so that only about $10 \%$ of the events were analyzed on-line. 


\section{DATA ANALYSIS}

This chapter deals with the transformation of date on tape into the elastic differential cross section and analyzing power.

\section{IV-A. CALIBRATION OF DETECTOR SYSTEM}

Before cross section data could be obtained, the quantities used to obtain the final result have to be calibrated. In particular, it was important to calibrate the polar scattering angle $\theta$, especially since the out of plane scattering angle which is used to calculate $\theta$ (see Appendix A) can vary dramatically in the small angle region.

After the voltage settings for the chambers were determined, the chambers were then calibrated on-line (and later off-line) to give the correct position information and subsequently software aligned. The spectrometer was then positioned to $9.2^{\circ}$ and $\theta$ callbrated. Actually, since $\theta$ is calculated according to eqn. (5) of Appendix A, $\theta_{\mathrm{H}}$ and $\theta_{\mathrm{V}}$ were calibrated, ie. the horizontal and vertical scattering angles at the target respectively.

In order to calibrate $\theta_{V}$, a horizontal wire was used for a target along with horizontal slits at the spectrometer entrance to define $Q_{V}$. This is shown schematically in figure 30 . Particles hitting the wire $\left({ }^{56} \mathrm{Fe}\right)$ target and passing through the slits give trajectories of known $\theta_{v}$. From figure $30, \theta_{v}$ is given by 


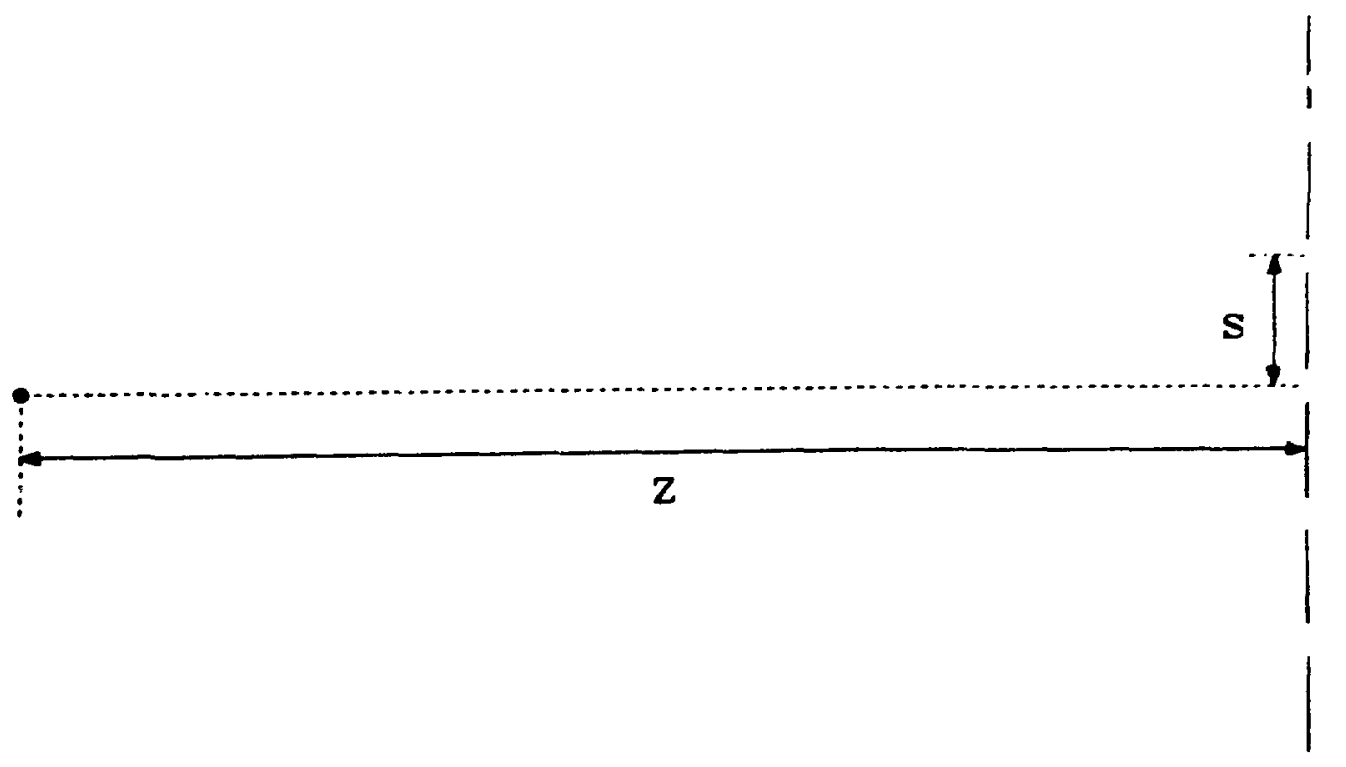

Fig. 30

Wire and slit conflguration for calibratir.g $Q_{v}$. $z=79.22 \mathrm{~cm}, s=1.27 \mathrm{~cm}$.

$$
\theta_{v}=\tan ^{-1}\left(\frac{n s}{2}\right) \quad n=0, \pm 1, \pm 2, \ldots
$$

By changing the fields of the hRS dipoles, the position dependence on the focal plane with $Q_{v}$ is determined. Figure 31 shows a plot of $Q_{v}$ vs. $x$ at the focal plane in wich $Q_{\text {p }}$ has been calculated from a rough estimate of the coefficients used to define it. These coefficients are described below. The boxes in the figure correspond to trajectories of known $Q_{V}$. For each trajectory of known $Q_{V}$, four quantities measured at the focal plane vere vritten to a file. The file containing these trajectories was then used to construct an expression for $Q_{v}$ in terms of polynomials of the neasured focal plane quantities. The measured focal plane quantities are: $x_{F}$, the $x$ position at the focal plane, $\theta_{F}$, theta at the focal plane, $y_{F}$, the $y$ 


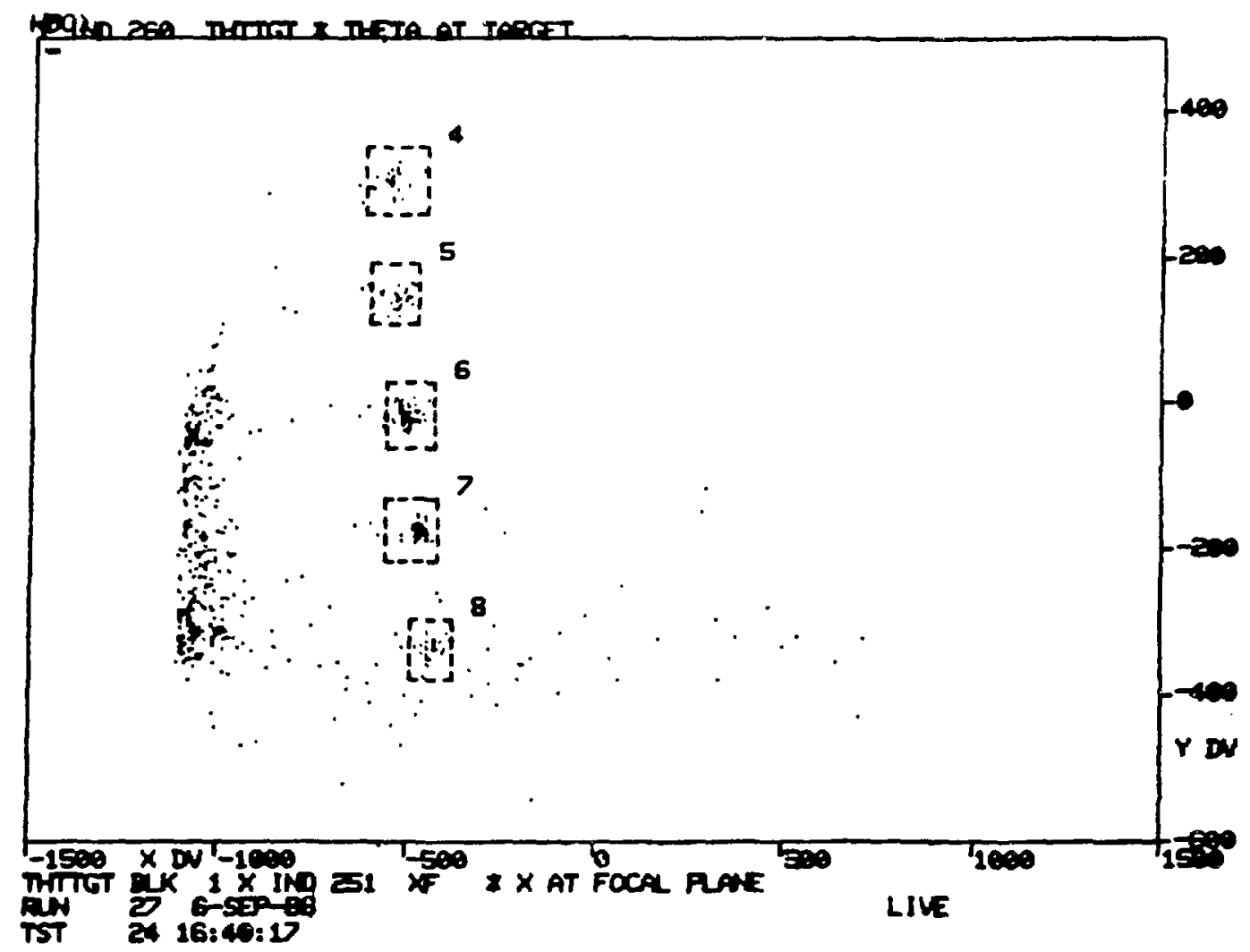

Fig. 31

Vertical scattering angle at the target vs. $x$ at the focal plane.

position at the focal plane, and $\phi_{F}$, phi at the focal plane. $x_{F}$ is defined as the mean position of the $x$ positions of chambers 1 and 2 $\langle X 12\rangle$. Similarly, $y_{F}$ is $\langle Y 12\rangle, \theta_{F}$ and $\phi_{F}$ are

$$
\begin{aligned}
& \theta_{F}=21.74(\langle X 34\rangle-\langle X 12\rangle) \\
& \phi_{F}=21.74(\langle Y 34\rangle-\langle Y 12\rangle)
\end{aligned}
$$

The trajectories were fitted to a polynomial of the form

$$
Q_{V}=c_{1}+c_{2} x_{F}+c_{3} \theta_{F}+c_{4} y_{F}+c_{5} \phi_{F}+c_{6} x_{F}^{2}+
$$




$$
c_{7} x_{F} \theta_{F}+c_{8} \theta_{F}^{2}+c_{9} y_{F}^{2}+c_{10} y_{F} \phi_{F}+c_{11} \phi_{F}^{2}
$$

The coefficients determined from the fit are listed in table 5 . clearly, the coefficients of $x_{F}$ and $\theta_{F}$ give the dominant terms as expected.

The calibration of $\theta_{\mathrm{H}}$ was done in a similar fashion. A set of 3 vertical rods vere used for targets along with vertical slits to define $\theta_{\mathrm{H}}$. This is shovn schematically in f1gure 32. In addition to changing the dipole fields of the HRS corresponding to different ${ }^{56} \mathrm{Fe}$ excitations, as was done in the $\theta_{v}$ calibration, the beam was adjusted to hit the left, center, and right rods for each excitation. $\theta_{H}$ for each trajectory through the spectrometer is known from figure 32 to be

Table 5 - Coefficients for determining $\theta_{v}$.

$\begin{array}{lr}c_{1} & 0.553271 E+00 \\ c_{2} & 0.685825 E+00 \\ c_{3} & -0.809896 \mathrm{E}+00 \\ c_{4} & -0.144005 E-01 \\ c_{5} & -0.537495 E-01 \\ c_{6} & -0.363392 E-02 \\ c_{7} & 0.283181 E-02 \\ c_{8} & 0.206263 E-03 \\ c_{9} & -0.236155 E-01 \\ c_{10} & 0.113300 E-01 \\ c_{11} & -0.297540 \mathrm{E}-04\end{array}$




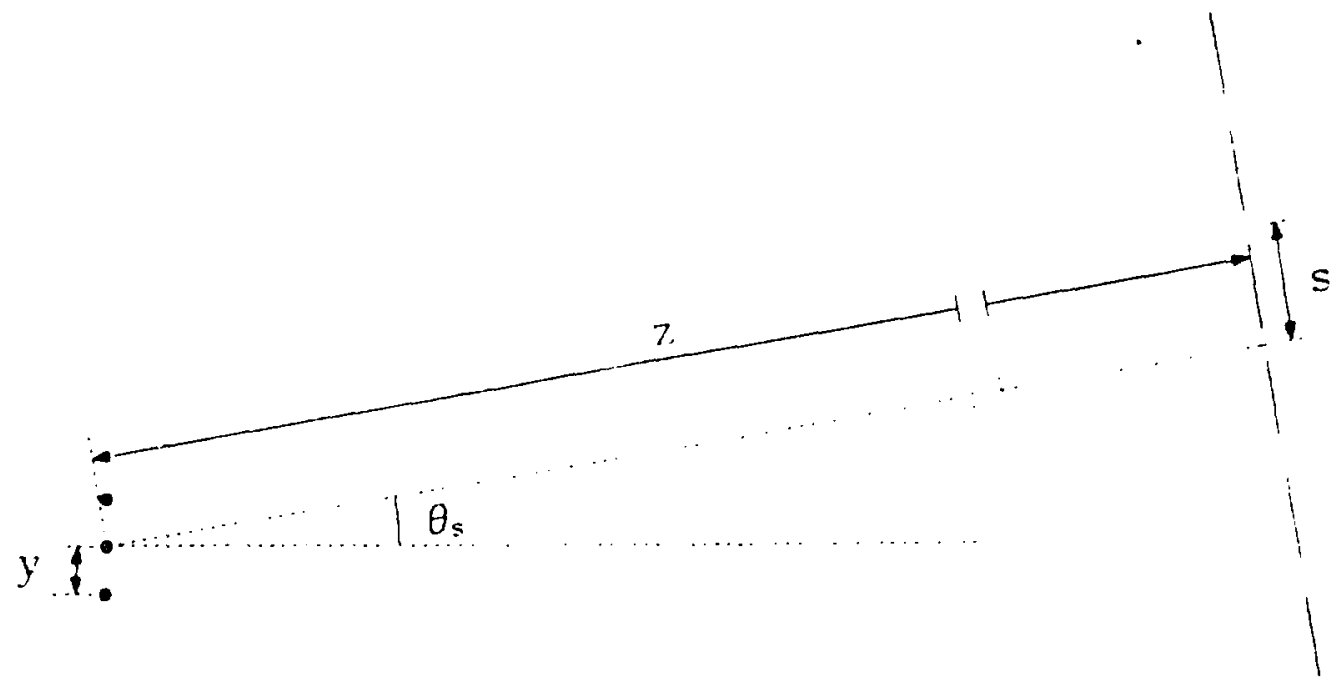

Fig. 32

Wire and slit configuration for calibrating $\theta_{\mathrm{H}}$.

$z=79.22 \mathrm{~cm}, s=1.27 \mathrm{~cm}, y=0.5 \mathrm{~cm}, \theta_{s}=9.2^{6}$.

$$
\theta_{\mathrm{H}}=A \tan ^{-1}\left(\frac{n s+A B \Delta s}{z^{\prime}}\right) \quad n \geq 0
$$

with

$$
\begin{gathered}
z^{\prime}=z+B y \sin \theta_{S} \\
\Delta s=y \cos \theta_{S}
\end{gathered}
$$

and

$$
\begin{aligned}
A=+1 & \text { left vertical slits } \\
-1 & \text { right vertical slits } \\
B=+1 & \text { right rod } \\
0 & \text { center rod } \\
-1 & \text { left rod }
\end{aligned}
$$


For each trajectory of known $\theta_{H}$, the measured values of $x_{F}, \theta_{F}, y_{F}$, and $\phi_{F}$ were written to a file which was used to construct a function for $\theta_{\mathrm{H}}$ in terms of these measured quantities. The polynomial describing $\theta_{\mathrm{H}}$ is

$$
\begin{gathered}
\theta_{H}=c_{1}+c_{2} x_{F}+c_{3} \theta_{F}+c_{4} y_{F}+c_{5} \phi_{F}+c_{6} x_{F}^{y} y_{F}+ \\
c_{7} x_{F} \phi_{F}+c_{8} \theta_{F} y_{F}+c_{9} \theta_{F} \phi_{F}
\end{gathered}
$$

with the fitted coefficients given in table 6 .

Once $\theta_{\mathrm{H}}$ and $\theta_{V}$ were calibrated, $\theta$ was found according to eqns. (i58) and (159) through eqn. (5) of Appendix A. However, the position of the spectrometer corresponding to absolute $0^{\circ}$ had to be known. This was found by setting the HRS at $0^{\circ}$ and allowing a low intensity pencil beam to go up on the focal plane. With no target in the scattering chamber, a histogram of the scattering angle was found

Table 6 - Coefficients for determining $\theta_{\mathrm{H}}$.

$\begin{array}{lr}C_{1} & 0.916273 E+00 \\ c_{2} & 0.244774 E-01 \\ c_{3} & 0.211819 E-01 \\ C_{4} & -0.496654 E+01 \\ c_{5} & -0.390232 E-01 \\ c_{6} & 0.581439 E-01 \\ c_{7} & 0.618709 E-02 \\ c_{8} & -0.393628 E-01 \\ c_{9} & -0.843100 E-02\end{array}$


to peak at $0.965 \mathrm{mr}$ with FUHM of about $0.178 \mathrm{mr}$. Thus the scattering angles measured in the cross section data had to be offset by this amount .

\section{IV-B. REPLAY OF THE DATA}

Using the cailbration constants of tables 5 and 6 , the cross section data was replayed with the computer code known as the GRS analyzer. The analyzer processes each event according to code written explicitly for each event type. In particular, scattered proton events identified as event 7 are processed in the analyzer with subroutine PROC7, while scaler events identified as event 8 are processed with subroutine PROC8. PROC7 takes all of the raw data, composed of $A D C$ and $T D C$ readouts, and uses it to calculate all quantities of interest. First, the time-of-flight (TOF) and pulse-height information is calculated from the scintillator TDC's and ADC's. This information is used to identify the particles passing through the detectors. A dot-plot of the ToF between scintillators 2 and 3 vs. the mean pulse height of scintillator 2 vas used for particle identification. Figure 33 shows such a dot-plot. A box was placed around proton events appearing in this dot-plot and only events falling within the TOF and wean puise height defined by the box were accepted as valid elastically scattered proton events. It was found that nearly all (>99\%) of the events Inside and outside of the box vere protons. This was not surprising, since the cross sections for producing other particles at these small 


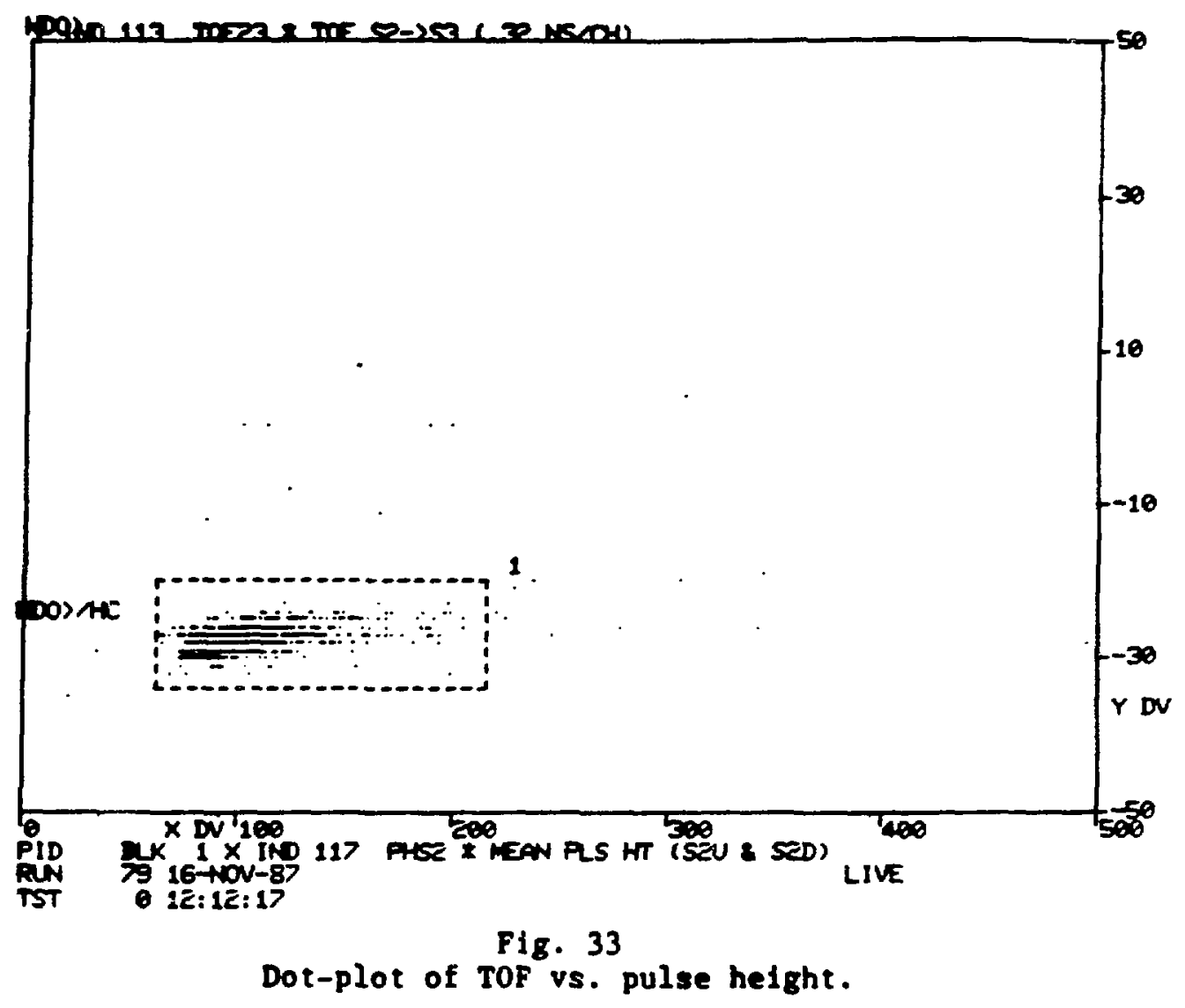

angles vith a $P / Z \pm 1 X$ of the elastic protons are much smaller than the p-nucleus elastic cross sections.

The chamber TDC ray data is used by PROC7 to obtain truncated anode vire positions and drift positions according to chamber calibration constants. This information is combined to give the coordinates of the points in space at which the scattered proton passed through each chamber. These coordinates are used to calculate quantities auch as $\langle x 12\rangle$ which are used in turn to calculate the focal plane quantities such as $\theta_{f}$ according to eqn. (157). These focal plane quantities are used as input to eqns. (158) and (159) to 
calculate $\theta_{\mathrm{H}}$ and $\theta_{\mathrm{V}}$, which are then used by egn. (5) of Appendix $A$ to calculate $\theta$. Thus there are many ( 100 ) quantities calculated from the - 30 pieces of raw data read from tape for each event 7.

After the o:antities of interest are calculated, various tests can be applied to them to determine under what category an event should be placed. For example, a standard good proton event is signified by: 1) all $x$ and $y$ position information from chambers 1-4 give a reasonable trajectory of the scattered particle, and 2) the scattered particle is a proton based on the test that the rof and pulse height information described above fall within the accepted range. This standard good event test is then used to look at histograms of things such as missing mass and scattering angle. The missing mass histogram, tested on a standard good event, is used to define a good event. A good event is a standard good event with the missing mass falling within prescribed limits. Because of the small angles involved in this experiment, the missing mass spectrum has only one peak and the allowed values of the missing mass used to define a good event were given by limits such that the peak of the spectrum fitted comfortably within the limits. Thus virtually all the particles in the missing mass spectrum were contained in the peak of the spectrum.

Before binning the data into scattering angle bins $3 \mathrm{mr}$ wide, one more test was applied to the good event test. As the out-of-plane scattering angle at these small scattering angles can be $90^{\circ}$ (see appendix A), the solid angle as a function of scattering angle varies dramatically. At large scattering angles, one can look at a 
histogram of the polar scatering angle without any solid angle corrections to get a good idea of what the angular distribution looks like. This is because the solid angle is roughly constant at large angles. However, the same histogram at small angles, shown in the top half of figure 34, does not display the correct angular distribution. The mantfestation of the rapidly varying solid angle in this histogram is quite apparent.

To see the correct angular distribution, a cut was made to make the solid angle variation from bin to bin very small. By restricting the vertical scattering angle at the target to $\pm 5 \mathrm{mr}$ instead of $\pm 2^{\circ}$ as given by the physical acceptance of the spectrometer, the region in which the solid angle varies rapidly is shifted to even smaller scattering angles. The maximum out-of-plane scattering angle given by eqn. (10) of appendix $A$ would then be described by making the transformation $2^{0} \rightarrow 5 m r$ everywhere in that discussion. Since the solid angle acceptance for each bin in this angular range is nearly constant with this cut, the correct angular distribution can therefore be seen. This is shown in the bottom of figure 34 . The yields for determining the differential cross section were obtained from binning the angular distribution as it appears in the lower half of figure 34 .

The position of the elastic spectral line on the focal plane was different for the different HRS spectrometer angle settings. Figure 35 shows the entire focal plane illuminated with quasi-elastic $p-{ }^{208} \mathrm{~Pb}$ events. The $x$ axis is the vertical position or momentum direction of the focal plane and the $y$ axis is the horizontal focal 
$H P L>A E C=T H T I N P /-E R$

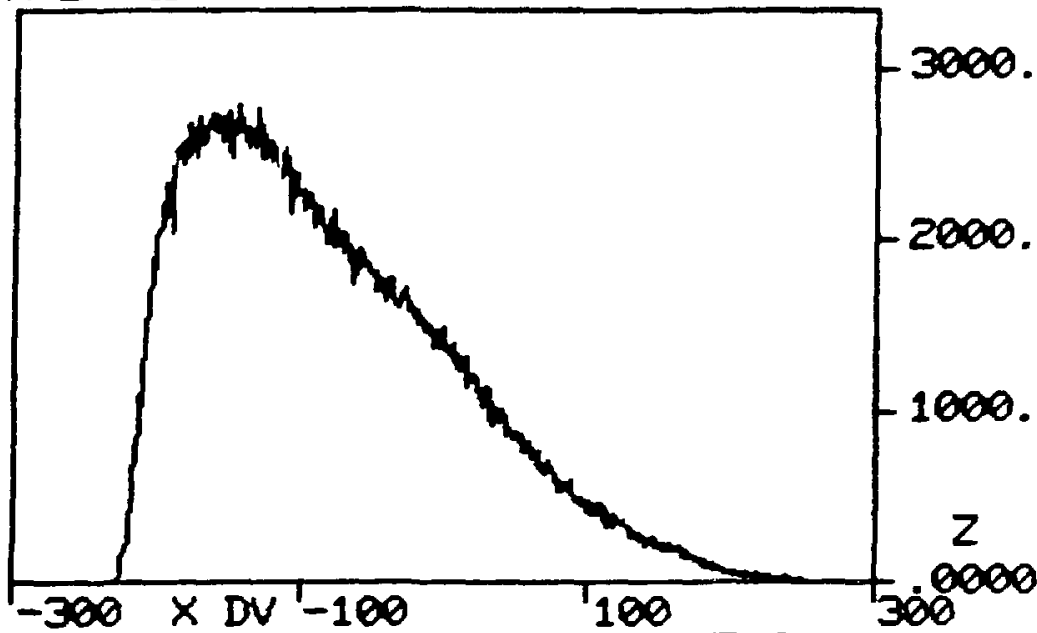

THTSCT BLK $1 \times$ IND 268 THTSCT *

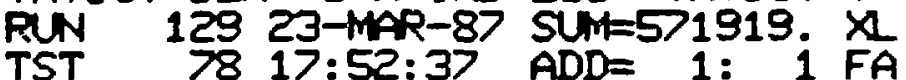

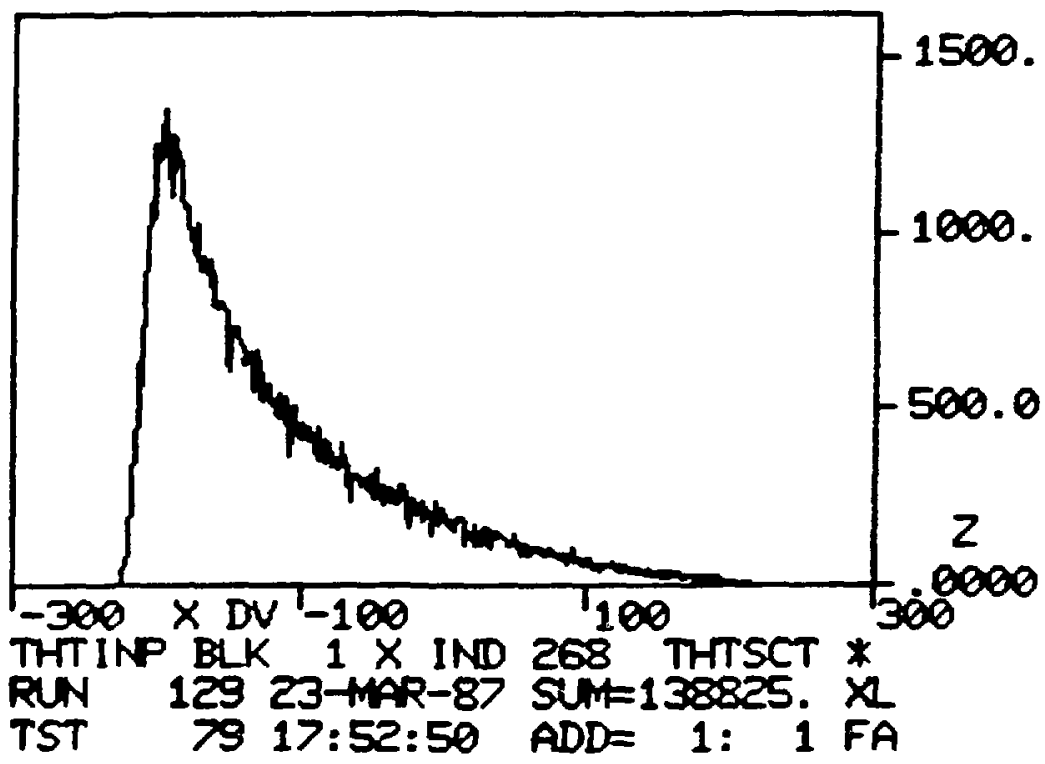

Fig. 34

The effect of restricting the vertical scattering angle at the target as explained in the text. 


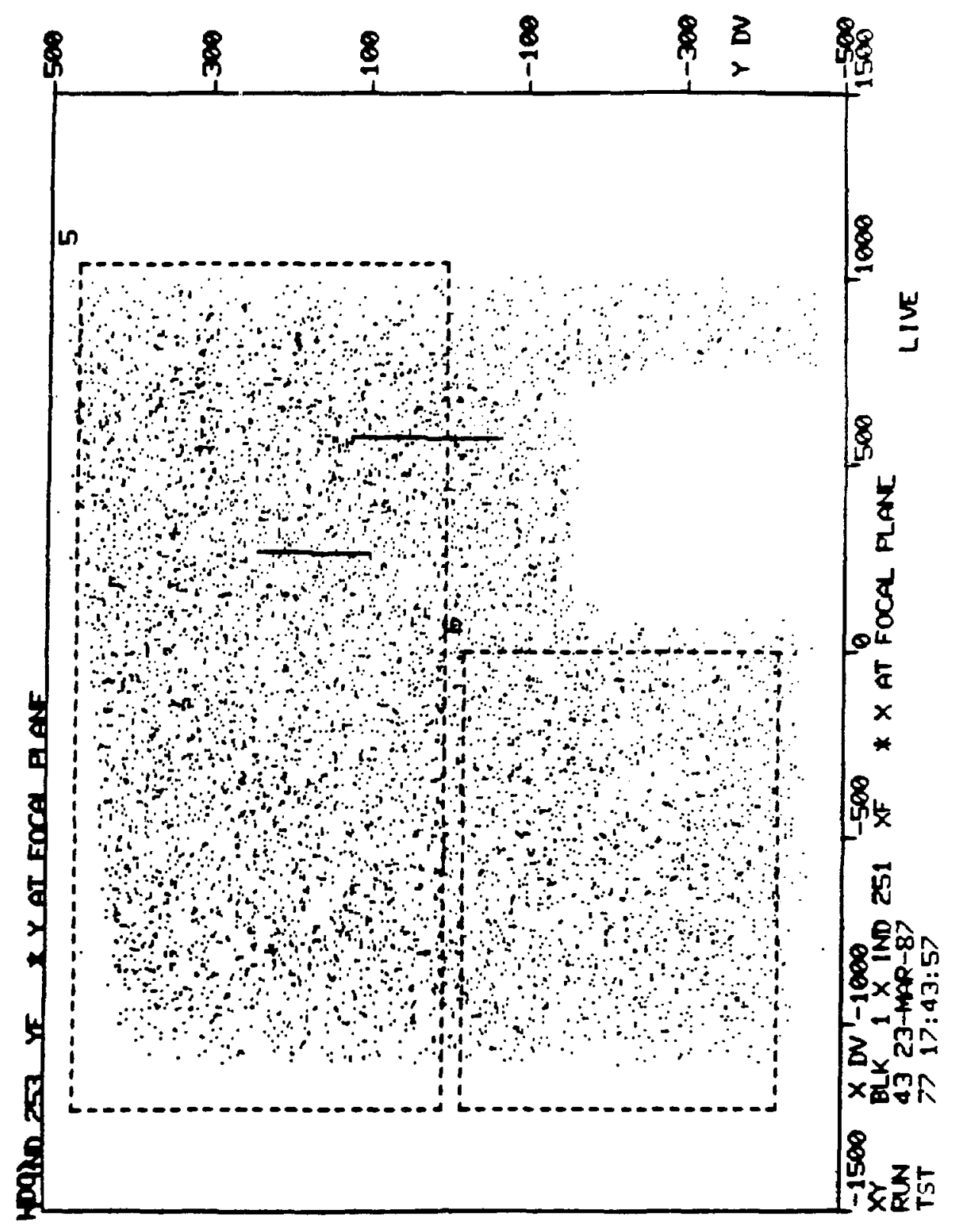

Fig. 35

View of the focal plane illuminated with p - $200 \mathrm{~Pb}$ quasi-elastic events. 
plane position or angular direction. The dead region for the beam to pass through can be seen quite clearly. This figure was used as a reference to place the elastic spectral line in the desired position.

With the spectrometer positioned at $-0.83^{\circ}$, the spectral line was as it appears in figure 36 . The cutoff from the position of scintillator $\mathrm{SX}$ can be seen quite clearly. For spectrometer angle settings of $2.5^{\circ}$ and $4^{\circ}$, the elastic spectral line was positioned below the dead region as shown in figure 37 in order to have the full angular acceptance of the spectrometer available. In contrast to figure 36 where the dead region is on the small angle side of the focal plane, the dead region is on the large angle side of the focal plane in figure 37. This is due to the crossover of scattered particles by the magnets which causes the small angle side of the focal plane to reverse itself with the large angle side in going from negative to positive spectrometer angle settings.

Because the drift chambers had been modified to allow the beam to pass through them, it was feared that chamber efficiency could have an angular dependence. Specifically, the question arose as to whether the chambers were less efficient for particles passing through near the dead region than they were for particles passing through far from the dead region. This would lead to a false angular distribution of the measured ylelds and a correction for this effect would be necessary.

To find out whether an angular dependent chamber efficiency existed, the following check was made. First each chamber plane was partitioned into areas corresponding to each scattering angle bin. 


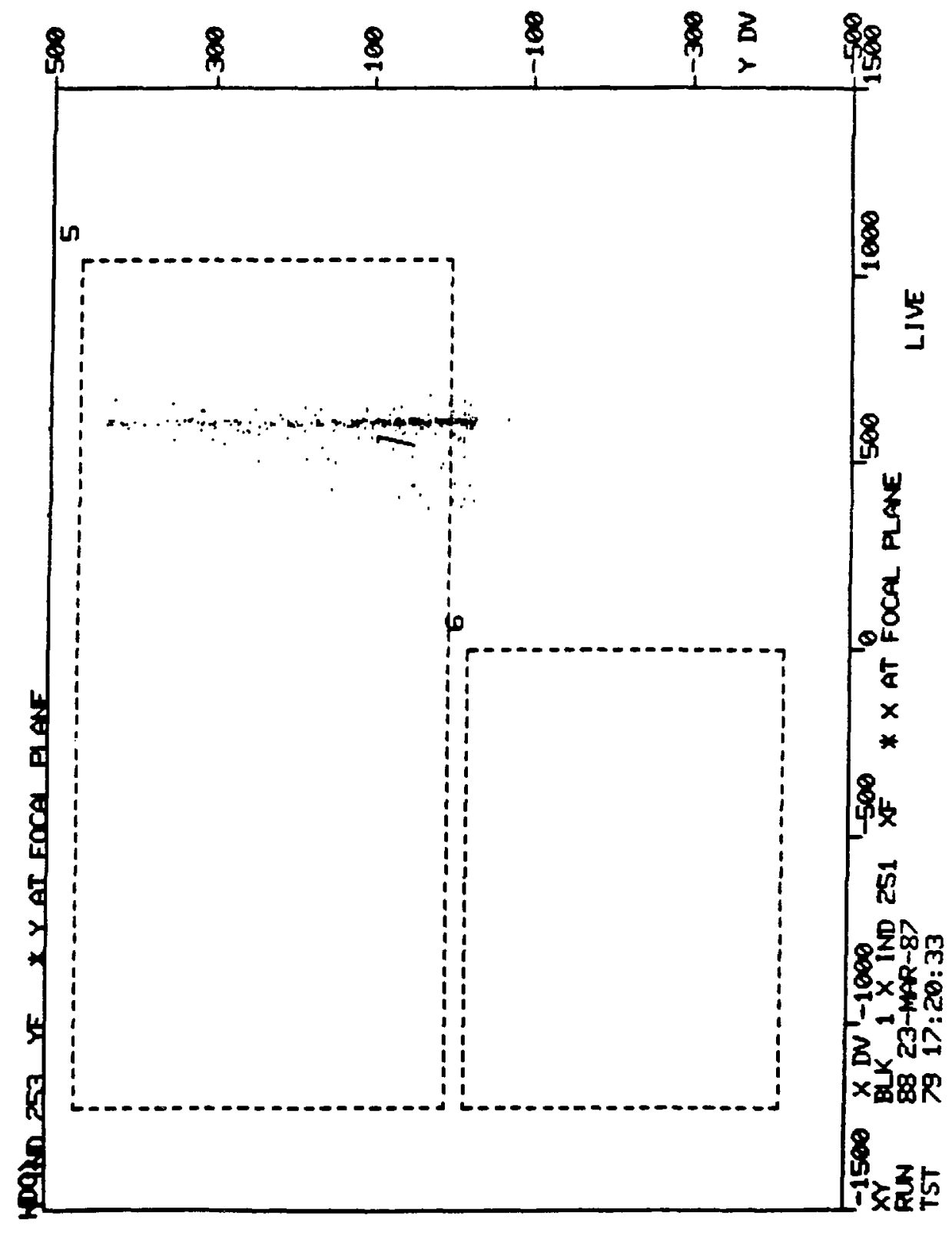

Fig. 36

Position of elastic line on the focal plane with the HRS at $-0.83^{\circ}$. 


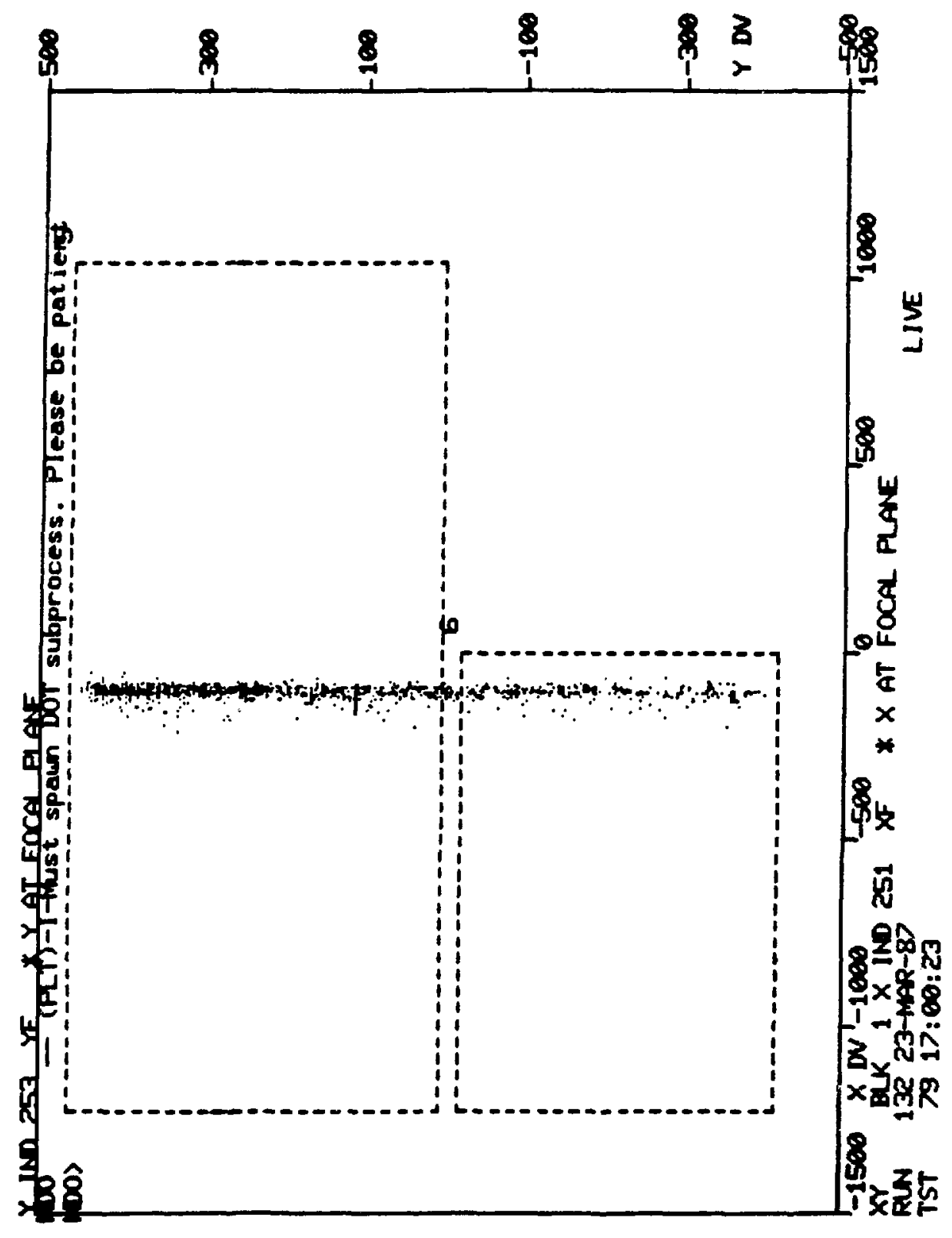

Fig. 37

Position of elastic line on the focal plane with the HRS at $2.5^{\circ}$. 


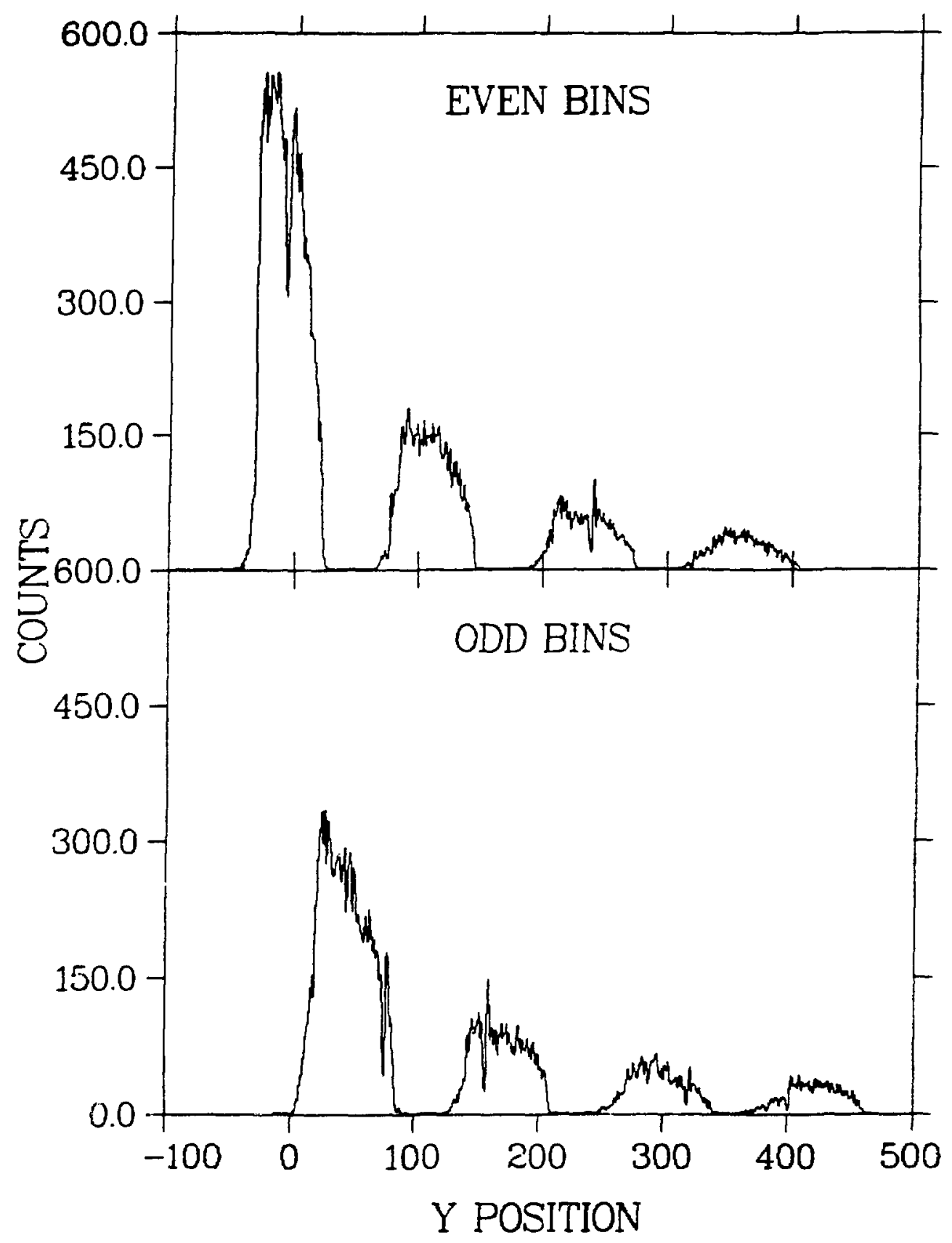

Fig. 38

Location of scattering angle bins in chamber $1 Y$. 
This is shown in figure 38 for the $Y$ plane of chamber 1 . As adjacent angular bins share some of the same space in the chamber, odd bins are displayed separately from even bins to show the position of each angular bin in the chamber more clearly. Thus the 8 peaks in the figure correspond to the locations in the $Y$ plane of chamber 1 of 8 different momentum transfers. These scattering angle locations were mapped out in all 8 chamber planes. Chamber efficiency for each chamber plane for each angular bin was then calculated. Efficiency for chamber $1 Y$ was defined as

$$
\text { EFF }=\frac{\text { All planes }}{\text { All planes except } 1 \mathrm{Y}}
$$

and similar expressions were used for the other 7 planes. The results are show in figures 39,40 , and 41 . Figure 39 shows the angular dependence of the $X$ planes and figure 40 that of the $Y$ planes. Figure 41 shows $X$ plane, $Y$ plane, and total chamber efficiencies as a function of momentum transfer. With the exception of chamber $1 Y$, no angular dependent chamber efficiency is observed. And when the chamber efficiency for each plane is combined to give the total chamber efficiency, the small angular dependence from chamber $1 Y$ appears to contribute very little. 


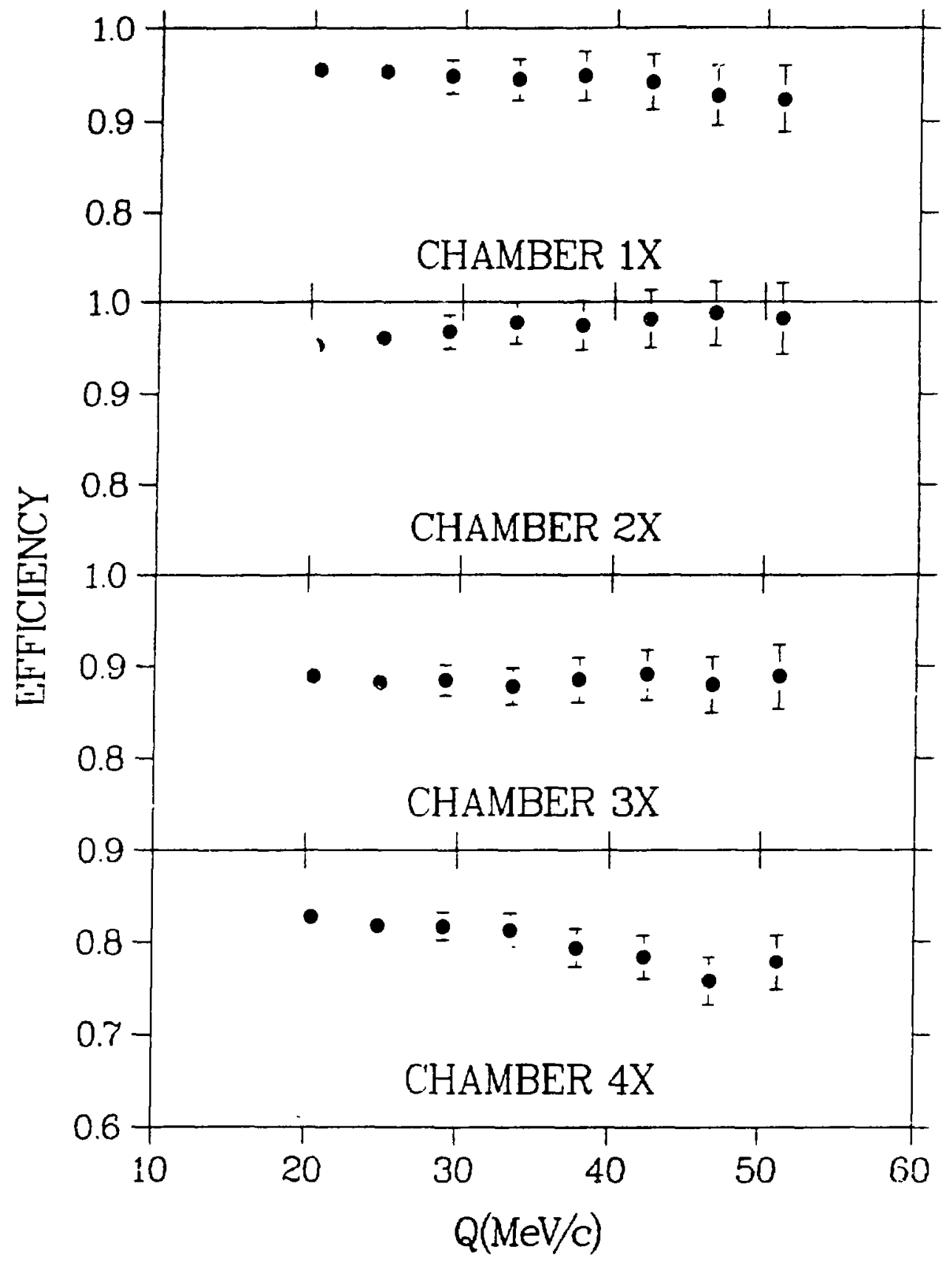

Fig. 39

Angular dependence of chamber efficiency for $X$ planes. 


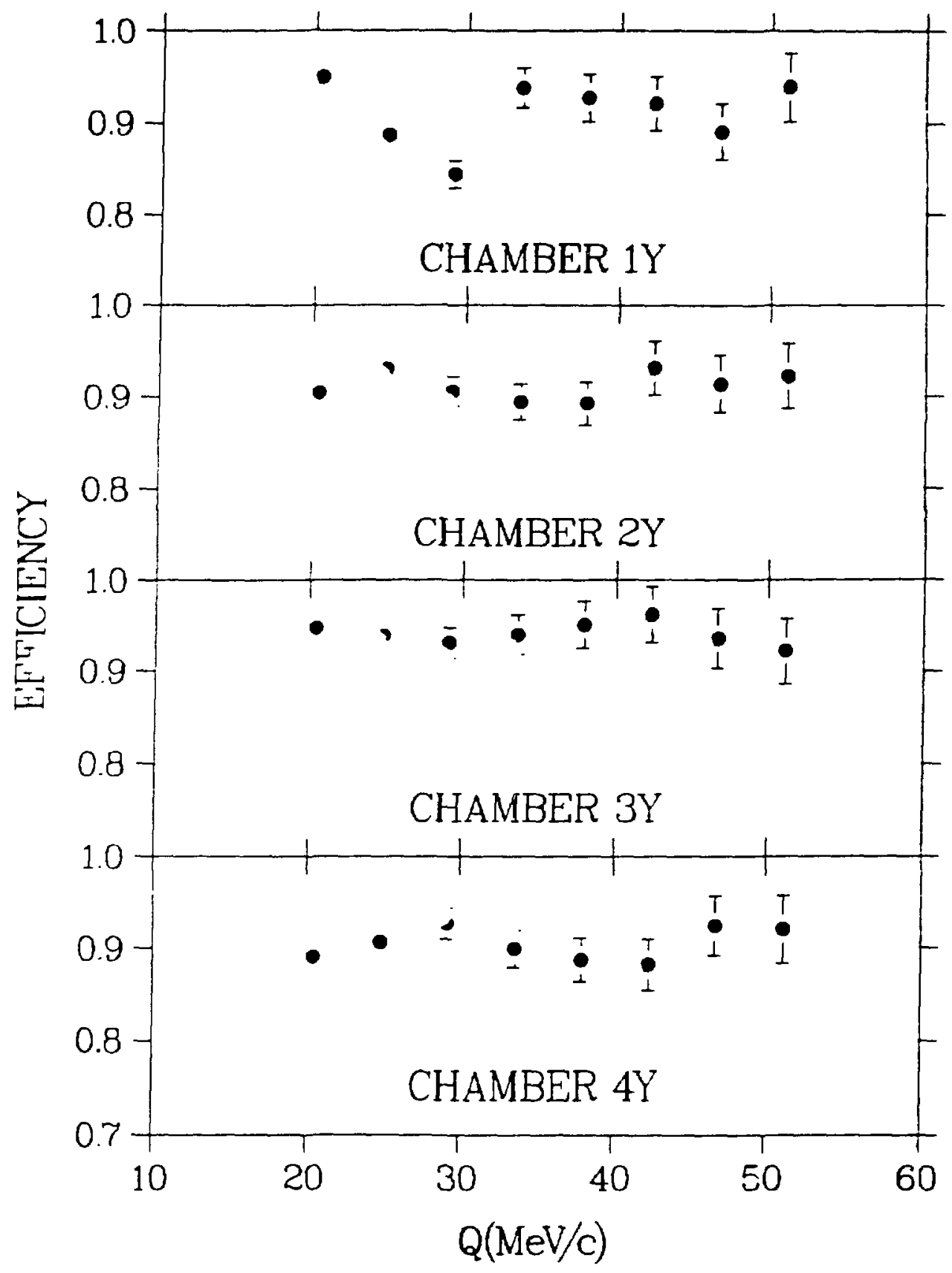

Fig. 40

Angular dependence of chamber efficiency for $Y$ planes. 


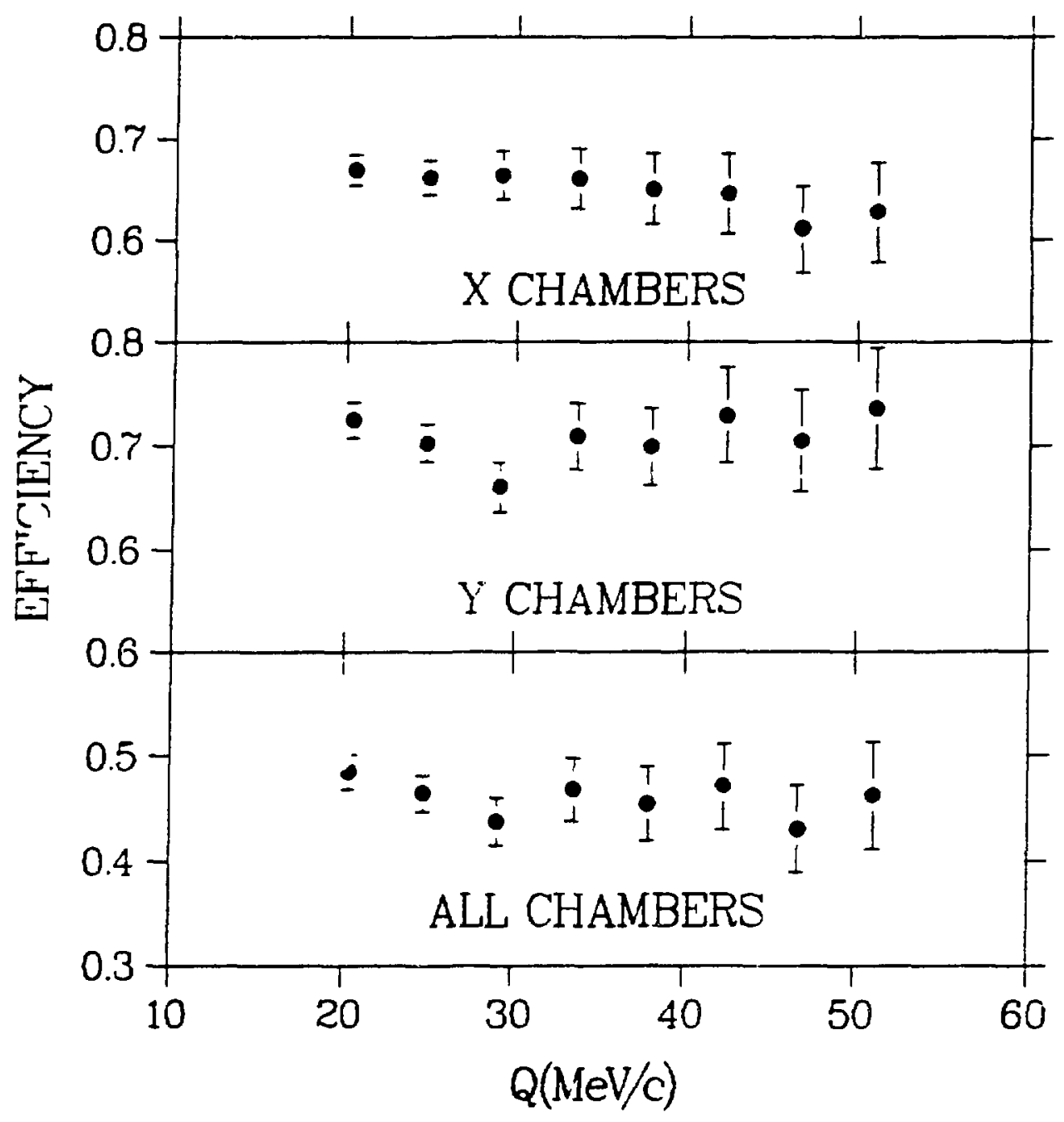

Fig. 41

$X, Y$, and total chamber efficiencies. 
IV-C. DETERMINATION OF CROSS SECTIONS AND ANALYZING POUERS

For each run replayed by the analyzer, output files containing test results and scaler sums were created. These files were then summed together for each run corresponding to the same target and angle. A program XSECT was written which read the summed output files and calculated the relative differential cross sections and analyzing povers from the test results and scaler sums in the file. The relative differential cross section vas calculated from the following expression:

$$
\frac{d g}{d Q}=\frac{G(\theta)}{\Delta Q(\theta)} \cdot\left(\frac{Y^{N}(\theta)}{C F N \star B E A M N}+\frac{Y^{R}(\theta)}{C F R \star B E A M R}\right)
$$

In this formula, $G(\theta)$ is the Jacobean of the transformation from lab to center-of-mass coordinates, $\Delta Q(\theta)$ is the solid angle subtended, $Y^{N}(\theta)$ and $Y^{R}(\theta)$ are the yields for normal and reverse polarized beam, CFN and CFR are normal and reverse correction factors, and BEAMN and BEAMR are the relative normal and reverse beam intensities. The correction factors take into account the efficiency of the system to detect events. Specifically, for normal polardzed beam

$$
\text { CFN }=\text { CEN`CLTN }
$$

where CEN is the chamber efficiency for normal beam and CLTN is the CAMAC live time corresponding to normal beam. A similar expression 
was used for the correction factor for reverse beam. CAMAC live times were typically $99 \%$ for the $-0.83^{\circ}$ data and about $85-90 \%$ for the larger angle data.

The measure of the relative beam intensity was done in two ways. For data taken at $2.5^{\circ}$ and $4.0^{\circ}$, the beam intensity was monitored with the counts from the line $C$ polarimeter. Namely, the relative beam intensity was taken to be the sum of the counts of the scintillators of each arm of the line $C$ polarimeter.

$$
\text { BEAMN = LCPOUN + LCPODN + LCPOLN + LCPORN }
$$

Here LCPOUN, LCPODN, LCPOLN, LCPORN are the counts in the up, down, left, and right arms of the line $C$ polarimeter for normal beam. A similar expression was used for the relative beam intensity for reverse beam. Fig. 42 shows the line $C$ polarimeter scintillator configuration. A $32 \mathrm{mil}$ scintillator target vas used for the polarimeter target at these angles. This target was chosen as it maximized the number of counts obtained from each arm of the polarimeter. Therefore, the polarization of the beam was not determined from the line $\mathrm{C}$ polarimeter as a standard $\mathrm{CH}_{2}$ target was not used. This method of monitoring the relative beam intensity was chosen over the method of using the ion chambers in the scattering chamber to measure the relative beam intensity because the scattered particles entering the spectrometer ist $2.5^{\circ}$ would have been intercepted by the frames of the ion chambers. Thus the use of the ion chambers at the spectrometer angle of $2.5^{\circ}$ was not possible. The 


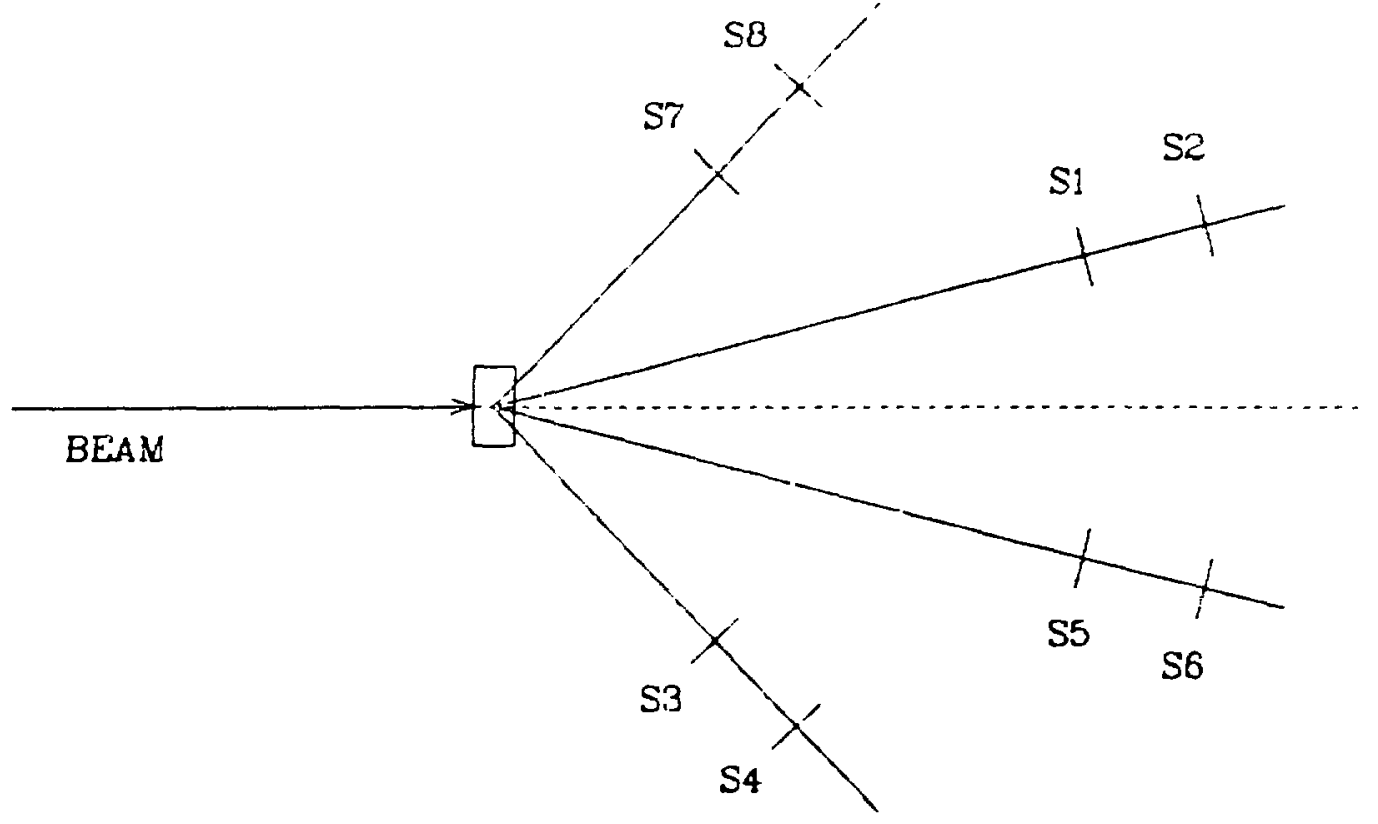

Fig. 42

Scintillator configuration for the line $C$ polarimeter in the up-dow'n plane. LCPOUN $=\mathrm{S} 1 \cdot \mathrm{S} 2 \cdot \mathrm{S3} \cdot \mathrm{S4}, \mathrm{LCPODN}=\mathrm{5} 5 \cdot \mathrm{S6} \cdot \mathrm{S7} \cdot \mathrm{SB}$. The left-right plane is similar.

ion chambers were used at $4.0^{\circ}$, but it was discovered after the experiment that the beam did not pass through the ion chambers, and therefore the line $C$ polarimeter information had to be used as the only alternative.

At $-0.83^{\circ}$ the relative beam intensity was measured with the ion chambers behind the focal plane. No polarimeter target was in place. As mentioned in the last chapter, there were large dark currents observed at this spectrometer angle setting. These dark currents were traced to the ion chambers becoming radioactive as the beam 
passed through them. This had to be taken into account in determining the relative beam intensity. The dark current was measured by putting the beam plugs in place in line $C$ to prevent beam from hitting the target, and observing the number of counts from the digitized charge integrator as a function of the number of beam gates. This was done for both ion chambers ER02 and ER04. It was found that the dark current per beam gate was about $7 \%$ of the beam current per beam gate, ie. the ratio of charge integrator counts without beam on target to with beam on target was $\sim 0.07$. Therefore several dark current runs were made to establish the dark current per beam gate which could be subtracted from the beam current per beam gate.

Figure 43 shows the dark current of ERO2 per beam gate as a function of run number (or time) for normal polarized beam. The dark current per beam gate was taken to be the veighted average of the points in figure 43. The values determined for normal and reverse polarized beam were found to be

$$
\begin{aligned}
& \text { DARKN }=1.105 \times 10^{-3} \pm 0.035 \times 10^{-3} \text { counts/beam gate normal } \\
& \text { DARKR }=1.110 \times 10^{-3} \pm 0.037 \times 10^{-3} \text { counts/beam gate reverse }
\end{aligned}
$$

The dark current per beam gate of ER04 was also measured. However, the dark current determined from ER0 4 varied considerably more than that from ERO2. Therefore, it was decided to use ERO2 for measuring the relative beam intensity. The beam intensity used in eqn. was calculated as 


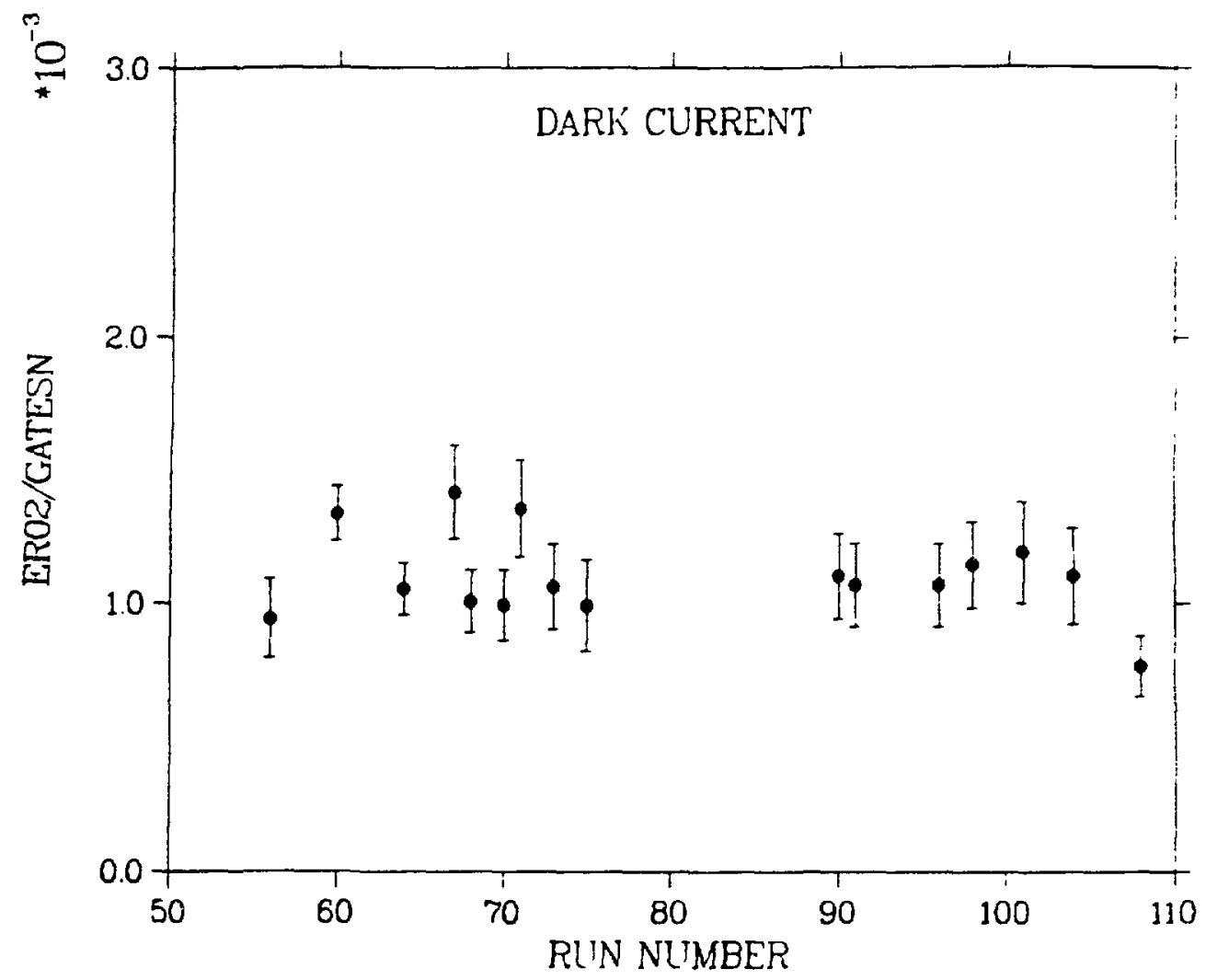

Fig. 43

Dark current per beam gale of ERO2 for normal beam.

$$
\text { BEAMN }=\text { ERO2N }-(\text { DARKN } \star G A T E S N)
$$

where ERO2N is the number of counts from the charge integrator for normal beam and GATESN is the number of beam gates for normal beam. A similar expression was used for BEAMR.

The analyzing powers were calculated from the formula

$$
A_{y}=\frac{1}{P}\left(\frac{N-R}{\bar{N}+R}\right)
$$


where

$$
N=\frac{Y^{N}(\theta)}{C F N \star B E A M N}, \quad R=\frac{Y^{R}(\theta)}{C F R \star B E A M R}
$$

and $P$ is the polarization of the beam. The beam polarization was determined from the BRS event quench. Namely, if the quenched beam intensity is $I_{Q}$ and the polarized beam intensity is $I_{P}$, then

$$
P=1-\frac{I_{0}}{I_{P}}
$$

The polarized beam intensity is given by

$$
I_{P}=\frac{S N}{G A T E S N}+\frac{S R}{G A T E S R}
$$

where SN and SR are the number of normal and reverse events scaled during the polarized cycle. Similarly, $I_{Q}$ is

$$
I_{0}=\frac{S O}{\text { GATESO }}
$$

where $S O$ is the number of events scaled during the quenched cycle, and GATESO is " : number of beam gates during the quenched cycle. This method as chosen as the line $\mathrm{C}$ polarimeter was not operating with a $\mathrm{CH}_{2}$ target and the line $\mathrm{B}$ polarimeter, from which beam polarization information could have been obtained, was not operating. 
However, the polarization glven by the HRS event quench agreed quite well with the polarization determined from the quenches of the up and down arms of the line $C$ polarimeter counters.

Because no calibration of the ion chambers was performed due to time limitations, the absolute normalization was determined from existing data. Namely, the absolute normalization constants were calculated using $p-{ }^{208_{\mathrm{Pb}}}$ data of the University of Texas 1,10 . This was done as follows. An interpolation function for the differential cross section as a function of momentum transfer $q$ for the Texas data was found from the ralation ${ }^{19}$

$$
\frac{d \sigma}{d Q}(q)=a_{1}+\sum_{j=2}^{n}\left(a_{j} \prod_{1=1}^{j-i}\left(\Delta-A_{1}\right)\right)
$$

where

$$
\Delta=\frac{q-q_{1}}{q_{2}-q_{1}}, \Delta_{i}=\frac{q_{i}-q_{1}}{q_{2}-q_{1}}
$$

and

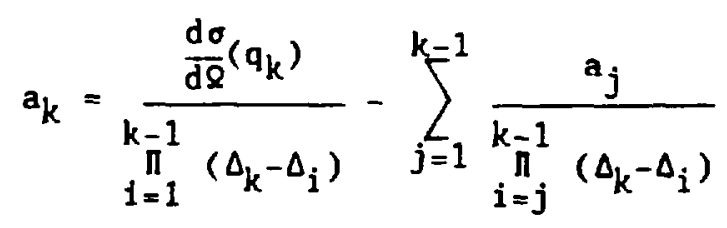

The value of $\boldsymbol{n}$ used for the interpolation function was chosen such that the normalization constant determined from the interpolation function changed less than a percent from using $n-1, n$, and $n+1$ terms. For most cases, $n$ was given the value of 7 . A normalization 
constant was determined for each data point in the region of overlap between the data and the Texas data by taking the ratio

$$
N(q)=\frac{\left(\frac{d \sigma}{d \Omega}(q)\right)^{\text {Texas }}}{\frac{d \sigma}{d \Omega}(q)}
$$

The final normalization constant was then taken to be the weighted average of the normalization constants determined from each individual data point:

$$
N=\frac{\sum\left(\frac{N(q)}{\sigma^{2}(q)}\right)}{\sum\left(\frac{1}{\sigma^{2}(q)}\right)} \pm\left(\frac{1}{\sum\left(1 / \sigma^{2}(q)\right.}\right)^{1 / 2}
$$

where the summations are over all $q$ in the region of overlap. The interpolation errors $\sigma(q)$ where determined from the statistical errors of the data points. The error in the final normalization constant varied from $0.4 \%$ to $0.9 \%$ in normalizing the data for the three spectrometer angle settings. Table 7 lists the normalization

Table 7 - Normalization constants from Texas $\mathrm{p}-{ }^{208} \mathrm{~Pb}$ data. 
constants for the three angular settings of the HRS as determined from the $\mathrm{p}-{ }^{208} \mathrm{~Pb}$ Texas data.

Once the normalization constants for each angle of the ${ }^{208} \mathrm{~Pb}$ data were determined, the normalization constants for the ${ }^{12} \mathrm{C}$ and ${ }^{40} \mathrm{Ca}$ data could then be determined using the relation

$$
\begin{aligned}
& \left(\frac{d \sigma}{d Q}\right)_{X}^{A}=\left(\frac{d \sigma}{d Q}\right)_{P b}^{A} \frac{\left(\frac{d \sigma}{d Q}\right)_{X}^{R}}{\left(\frac{d \sigma}{d Q}\right)_{P b}^{R}} s_{X} \\
& =\left(\frac{d \sigma}{d \bar{Q}}\right)_{X}^{R} S_{X}
\end{aligned}
$$

The superscript A signifies an absolute differential cross section, and $R$ that of a relative differential cross section. $X$ corresponds to either the ${ }^{12} \mathrm{C}$ or the ${ }^{40} \mathrm{Ca}$ target. $S_{x}$ is the ratio of the number of ${ }^{208} \mathrm{~Pb}$ nuclei per unit area to the number of $X$ nuclei per unit area in the ${ }^{208} \mathrm{~Pb}$ and $X$ targets respectively. Using the target thicknesses of $100.0 \pm 1.0 \mathrm{mg} / \mathrm{cm}^{2}$ for ${ }^{208} \mathrm{~Pb}, 73.6 \pm 0.5 \mathrm{mg} / \mathrm{cm}^{2}$ for ${ }^{40} \mathrm{Ca}$, and $139.0 \pm 1.0 \mathrm{mg} / \mathrm{cm}^{2}$ for ${ }^{12} \mathrm{C}$, the values of $\mathrm{s}_{\mathrm{X}}$ are

$$
\begin{aligned}
& S_{C a}=0.26282 \pm 0.00263 \\
& S_{C}=0.04167 \pm 0.00065
\end{aligned}
$$

Figs. 44 and 45 show the differential cross section and analyzing power data for the three targets as a function of momentum transfer $q$. The data are binned in angular bins of $3 \mathrm{mr}$ in width. 


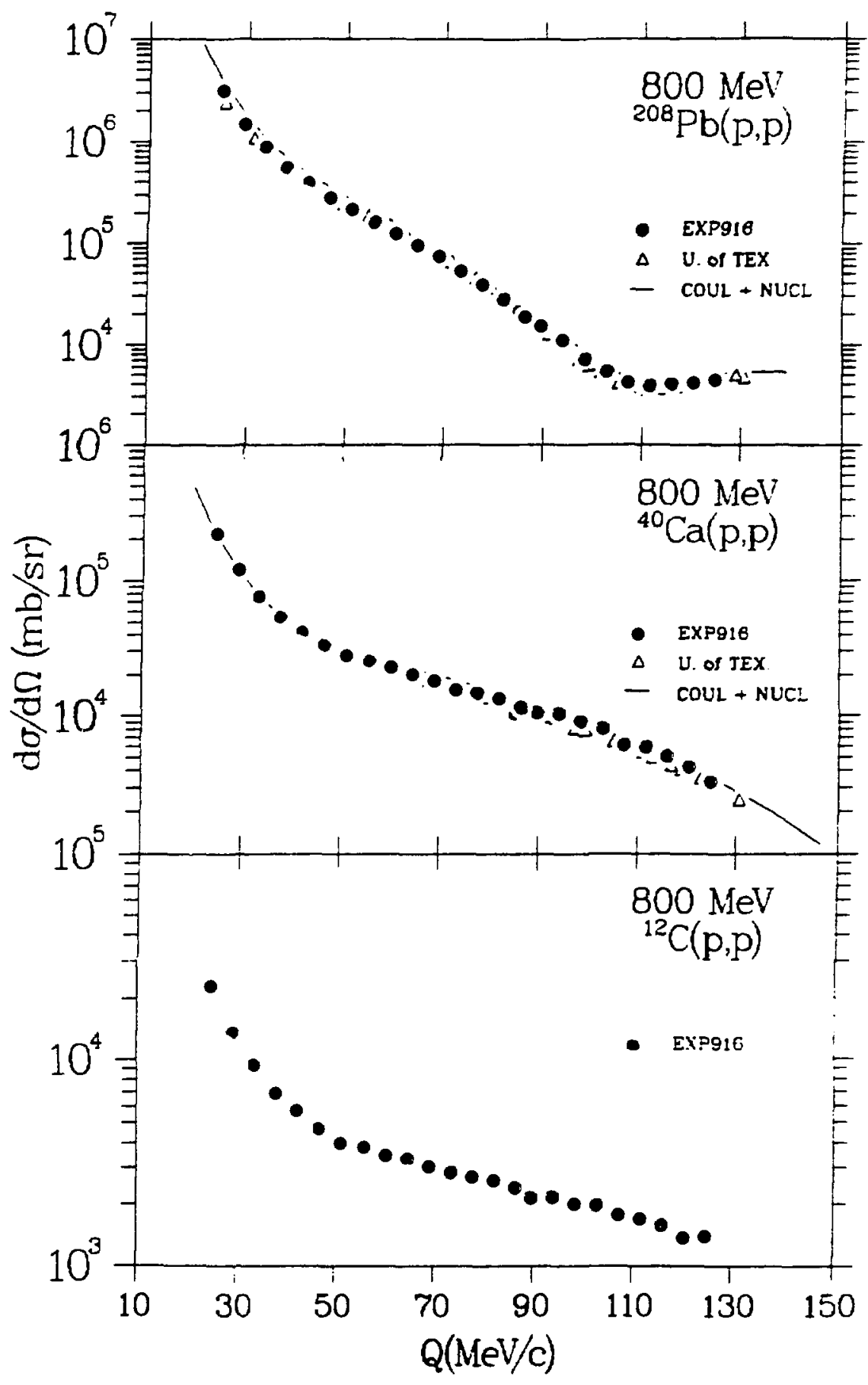

Fig. 44

Differential cross sections for protons on $12 \mathrm{C}$, ${ }^{10} \mathrm{Ca}$, and $200 \mathrm{~Pb}$ at $800 \mathrm{MeV}$. 


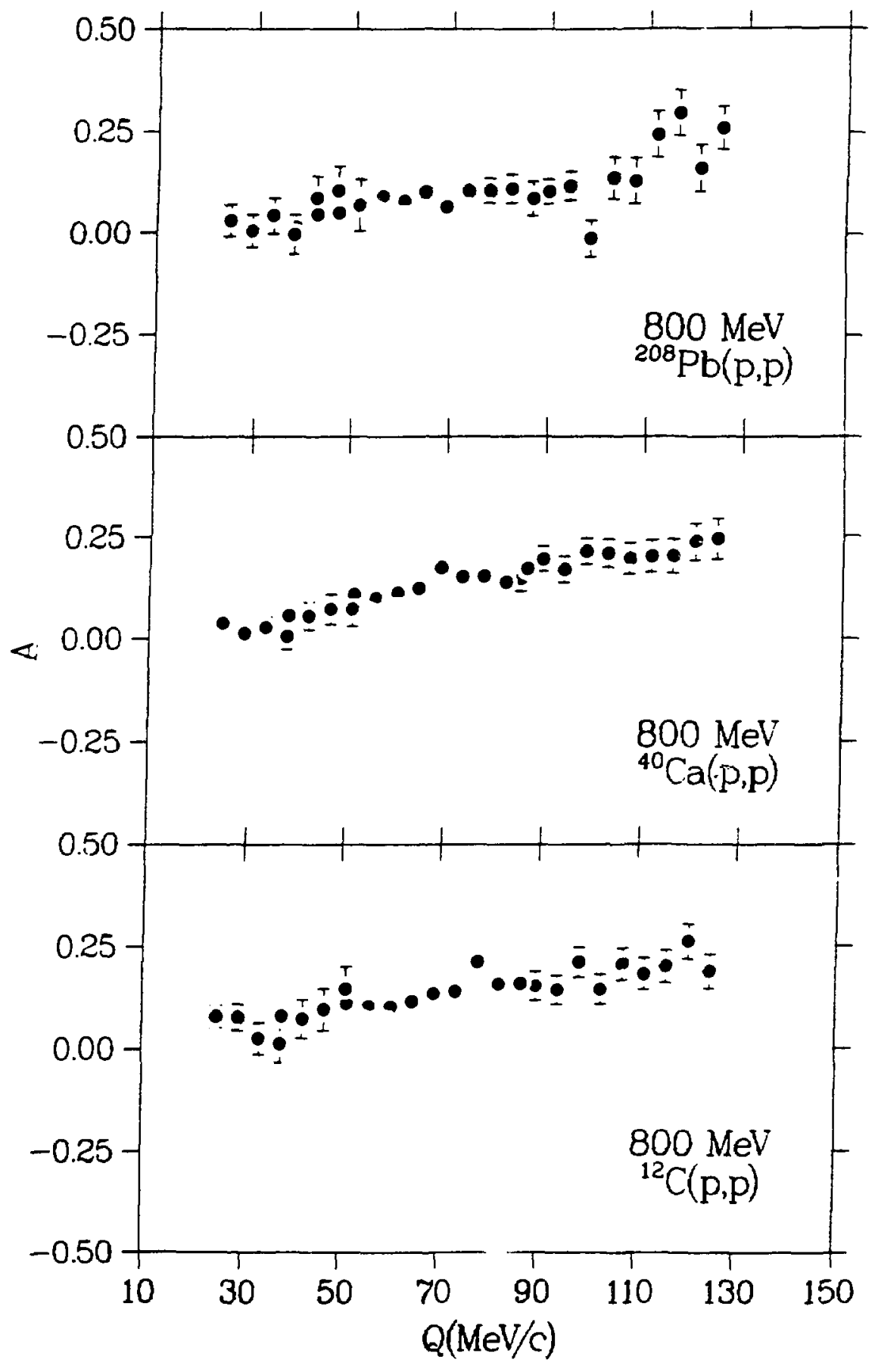

Fig. 45

Analyzing powers for pretons on $12 \mathrm{C}$, ${ }^{40} \mathrm{Ca}$, and $208 \mathrm{~Pb}$ at $800 \mathrm{MeV}$. 
The solid black circles represent the data obtained in this experiment labeled EXP916 for LAMPF experiment 916. The triangles represent data of the University of Texas'7,12120. The solid line represents a theoretical calculation to be discussed in the next section. The error bars included with the data points in fig. 44 are purely statistical and are therefore too small to be seen in the figure. The numerical values of the data are tabulazed in appendix D.

The errors contributing to the differential cross section stem from uncertainties in each quantity used in the calculation of the cross section as given by eqn. (160). Determination of the error each quantity introduces can then be used to find the total error in the cross section from the relation

$$
(\delta \sigma)^{2}=\sum_{i}\left(\frac{\partial \sigma}{\partial j}\right)^{2}(\delta 1)^{2}
$$

Here $\sigma$ is the differential cross section and the sum is over each of the $i$ quantities in eqn. (160), i.e. G, $\Delta Q, Y^{N}, Y^{R}$, CFN, CFR, BEAMN, and BEAMR.

The error in $G$ comes from the uncertainty in knowing the scattering angle. As mentioned previously, the error in the determination of the absolute zero of the scattering angle is about $.18 \mathrm{mr}$. In addition, the angular resolution is about $1 \mathrm{mr}$. Over this angular range at the angles considered here, the value of $G$ changes by less than 1 part in $10^{4}$. Thus, in comparison to the 
errors associated with the other quantities determining the cross section, the error $\delta G$ is small and can be neglected. The error in $\Delta Q$ comes from using empirical formulae such as eqn.(15B) to determine quantities used to calculate the solid angle. This error has been estimated ${ }^{21}$ at $\sim \pm(1-2) \%$.

The errors associated with the yields $Y^{N}, Y^{R}$ in general come from two sources: statistics and background. As previously mentioned, no significant background was detected and thus no error due to background has been included in the error of the yields. $\delta y^{N}$ and $\delta Y^{R}$ were simply given statistical errors for use in eqn. (168). The errors associated with the correction factors 8CFN and 8CFR come from the errors of the two quantities in the definition, eqn. (161). Uncertainties in chamber efficiencies and CAMAC livetime are much less the $1 \%$ and consequently the errors in the correction factors have been neglected.

The error in the measurement of the normal (reverse) relative beam intensity, SBEAMN ( $B$ BEAMR) depends on whether the beam intensity was monitored with the ion chambers as for the $-0.83^{\circ}$ data, or with the scintillators of the line $C$ polarimeter for the $2.5^{\circ}$ and $4.0^{\circ}$ data. For the scintillator case, the relative beam intensity was determined from eqn. (162) and the error for each of the four terms on the right side of eqn. (162) were added in quadrature to ohtain the total error in BEAMN (BEAMR). As the error associated with each scintillator arm is statistical in nature, statistical errors from the counts of each arm were taken to be the errors used in determining the total error in BEAMN (BEAMR). 
The ion chamber measurement of the relative beam intensity made use of eqn. (163) to take into account background due to tark current. The error in the normal (reverse) beam intensity is then found from the error in the counts from the integrator $\delta E R 02 N$ ( $\delta E R 02 R$ ) and the error in the dark current SDARKN (SDARKR). The determination of the dark current has already been discussed and since it is just scaling the counts from the integrator without beam, the error associated with it is essentially the error in the number of counts obtained from the integrator while the beam is of $f$. The uncertainty in the number of counts obtained from the charge integrator is not statistical, but fractional. This means that the error in the total number of counts will be \pm 1 count. However, the scalers that scale the number of counts put out by the integrator are inhibited by a pulse generated when the polarized beam enters the quench mode. Thus the scalers do not accept any counts put out by the integrator as long as the beam is in quench. Since the beam changes spin every two minutes, the total time that the beam is counted for a given polarization direction can be divided into two minute segments. As there are 9600 beam gates in a two minute period, the total number of counting segments $N$ for normal beam will be equal to GATESN/9600 rounded up to the nearest integer, and similarly for reverse beam. For each counting segment, the beam going through the ion chambers will cause the integrator to put out a certain number of counts that depends on how much charge was already in the integrator at the start of the counting interval. Thus the integrator will put out either the correct number of counts, one 
count too many, or one count too few for each counting segment. It can be shown that the probability $p_{0}$ of obtaining the correct number of counts from the integrator is $1 / 2$ and the prababilities of counting one too many or one too few, $p_{2}$ and $p_{-2}$, are each $1 / 4$. If $n_{-1}, n_{0}$, and $n_{1}$ are the occupation numbers corresponding to the probabilities $p_{-1}, p_{0}, p_{1}$ respectively, then

$$
N=n_{-1}+n_{0}+n_{1}
$$

The occupation numbers will be distributed according to the trinomial distribution 22

$$
\begin{aligned}
P\left(n_{-1}, n_{0}, n_{1}\right) & =\frac{N !}{n_{-1} ! n_{0} ! n_{1} !}\left(p_{-1}\right)^{n_{-1}}\left(p_{0}\right)^{n_{0}}\left(p_{1}\right)^{n_{1}} \\
& =\frac{N !}{n_{-1} ! n_{0} ! n_{1} !}\left(\frac{1}{2}\right)^{n_{0}\left(\frac{1}{\pi}\right)^{n_{1}+n_{-1}}}
\end{aligned}
$$

with the mean and variance for each case given by

$$
\begin{aligned}
\left\langle n_{1}\right\rangle & =N p_{i} \quad(i=-1,0,1) \\
\sigma_{i}^{2} & =N p_{i}\left(1-p_{i}\right)
\end{aligned}
$$

and the covariance of $n_{1}$ and $n_{j}$ is

$$
\sigma_{i j}^{2}=-N p_{i} p_{j}
$$


Clearly, the number of times that the integrator gives one count too many should be offset by the number of times that it gives one count too few. If $\Delta C=n_{1}-n_{-1}$ then the error in the total number of counts from all intervals combined is just $\sigma_{\triangle C}$, or

$$
\begin{aligned}
\sigma_{\Delta C}^{2}=\left(\frac{\partial \Delta C}{\partial n_{1}}\right)^{2} \sigma_{1}^{2} & +\left(\frac{\partial \Delta C}{\partial n_{-1}}\right)^{2} \sigma_{-1}^{2}+2\left(\frac{\partial \Delta C}{\partial n_{1}}\right)\left(\frac{\partial \Delta C}{\partial n_{-1}}\right) \sigma_{1-1}^{2} \\
& =\sigma_{1}^{2}+\sigma_{-1}^{2}-2 \sigma_{1-1}^{2} \\
& =N\left(\frac{3}{16}+\frac{3}{16}+\frac{1}{8}\right)
\end{aligned}
$$

or

$$
\sigma_{\Delta C}=\left(\frac{N}{2}\right)^{1 / 2}
$$

As a check of the method of counting the beam vith the scintillators of the line $C$ polarimeter, ve can make the following comparison. Instead of normalizing the lead data to lead data from the University of Texas, ve can normalize the lead data to the calcium data of the University of Texas ${ }^{20}$ using an expression similar to eqn. (167). Comparison of the two normalization constants would then serve as an Indication as to the accuracy of using the line $C$ polarimeter scintillators as anitor of the relative beam Intensity. Replacing the subscripts $\mathrm{Pb}$ vith $\mathrm{Ca}$ in eqn. (167) and $\mathrm{X}$ now signifying $\mathrm{Pb}$, the ratio of the absolute to relative differential cross sections for $\mathrm{Pb}$ will then be $\mathrm{NS}_{\mathrm{X}}$, where $\mathrm{N}$ is the normalization constant determined by comparing the calcium data with the Texas 
calcium data using eqn. (166) and $S_{x}$ is the ratio of the number of calcium nuclet per unt area to the number of lead nuclet per unit area in the calcium and lead targets respectively. For $2.5^{\circ}, \mathrm{N}$ is found to be $592.02 \pm 2.94$ and $s_{x}$ is just $3.805 \pm 0.052$. NS $x$ is then $2252.55 \pm 31.66$. Comparing this number vith the value listed in table 7, we find that the difference in the two normalization constants is about $1.7 \%$. Thus ve have good cross comparison consistency between data normalized with the beam counted with the Ion chambers and data normalized with the beam counted by the scintillators of the line $C$ polarimeter.

As for the uncertainty in the absolute normalization of the data, this can only be as good as the data it is normalized to. As the Texas data has an uncertainty in the absolute normalization of $5-10 \%$, this is therefore the uncertainty in the absolute normalization of the data presented in this vork. Appendix D contains in tabular form the differential cross section and analyzing power data. Errors shown for the lead cross section include the errors discussed in this section, namely statistical errors in $\mathrm{Y}^{N}$ and $\mathrm{Y}^{\mathrm{R}}$, and the errors associated with BEAMN and BEAMR as has been discussed. For the calcium and carbon cross section data, the errors shown include additionally the errors in the target thicknesses since these cross sections are calculated from the lead data ut 11 iaing enn. (167) which aakes use $j \hat{i}$ the target thicknesses. Hultiple scattering contributions to the differential cross section, as discussed in appendix $B$, vere found to be negligible and no correction to the angular distribution vas necessary. 


\section{RESULTS AND CONCLUSIONS}

In this chapter we present the results of fits of the data to the theoretical description of the scattering process. Using the parameterization of the differential cross section as discussed in section $C$ of chapter II, values for $\alpha$ are obtained for the data. The significance of data at small angles is then discussed. In particular, we investigate the ability of small angle elastic scattering data to discern between various theoretical models. Finaliy, we discuss some of the problems in obtaining high quality small angle data and how these difficulties might be overcome.

V-A. EXTRACTING THE REAL PART OF THE NUCLEAR AMPLITUDE

To obtain the real part of the forward spin independent nuclear amplitude, ve make use of the parameterized form of the differential cross section ean.(123). However, before this expression can be used, a simple modification to the Coulomb cross section eqn.(120) must be made. As it is, eqn.(120) is only valid for nonrelativistic projectiles. Since $800 \mathrm{MeV}$ protons are relativistic, the relacivistic form for Coulomb scattering should be used. This is accomplished by simply multiplying the nonrelativistic Coulomb cross section by a factor $r^{2}$. It is also desirable to write egn.(123) in terms of numerical factors and the parameters with simple units. Putting in values for constants and factors of hc where appropriate we have 


$$
\begin{aligned}
& \frac{d \sigma}{d \Omega}=\frac{k^{2} \sigma^{2} \cot \left(1+\alpha^{2}\right)|w|^{2} \exp \left(-b_{1} q^{2} \times 10^{-6}\right)}{61485334.4} \\
& +10\left(\gamma_{1}{ }^{2}+\gamma_{2}{ }^{2}\right) \exp \left(-b_{3} q_{2} \times 10^{-6}\right) \sin ^{2} \theta+\frac{1557533.58 k^{2} \beta^{2} \gamma^{2}}{q^{4}}\left(1+r^{2} \sin ^{2} \theta\right) \\
& -\frac{(\alpha(\cos 2 \phi R e W+\sin 2 \phi \operatorname{Im} W)+(\sin 2 \phi R e V-\cos 2 \phi \operatorname{Im} W)) B \sigma_{\operatorname{tot}} k^{2} \exp \left(-b_{1} q^{2} \times 10^{-6 / 2}\right)}{3.1415 q^{2}} \\
& +\frac{7893.12 \beta k T\left(r_{1} \sin 2 \phi-r_{2} \cos 2 \phi\right) \sin ^{2} \exp \left(-b_{3} q^{2} \times 10^{-6} / 2\right)}{q^{2}}
\end{aligned}
$$

where $d \sigma / d \rho$ is in $m b / s r, q$ and $k$ are in $M e V / c$ and the parameters $\sigma_{\text {tot }}, b_{1}, b_{3}, r_{1}$, and $r_{2}$ are in units of $\mathrm{mb},(\mathrm{GeV} / \mathrm{c})^{-2},(\mathrm{GeV} / \mathrm{c})^{-2}, \mathrm{fm}$ and $f m$ respectively. $B, T$ and the parameter $\alpha$ are dimensionless. The expressions for $B, T$ and $\phi$ will be

$$
\begin{gathered}
\beta=\frac{.00736 \mu \mathrm{Z}}{\mathrm{P}_{\mathrm{lab}}} \\
T=\frac{1.145 \mathrm{k}^{2}}{M^{2}} \\
\phi \operatorname{Bln}\left(112.773 / \theta_{\mathrm{cm}} \mathrm{k}\right)
\end{gathered}
$$

with $M$ the mass of the proton in MeV and $\mu$ and $P_{1 a b}$ the reduced mass and laboratory momentum of the incident proton in $\mathrm{HeV}$ and $\mathrm{MeV} / \mathrm{C}$ respectively. For the correction factor, we will take $R=E$ so that $W$ is just 


$$
H=(1-1 \beta / 2)^{-1}
$$

The method used to fit the data to eqn.(169) is that of minimizing $x^{2}$ with respect to all the free parameters involved. For each parameter $n_{k}$ allowed to vary in the fit $x^{2}$ is minimized according to the relation

$$
\frac{\partial x^{2}}{\partial n_{k}}=\frac{\partial}{\partial n_{k}} \sum_{i}\left(\frac{\sigma_{i}-\sigma\left(q_{i}\right)}{\varepsilon_{i}}\right)^{2}=0
$$

Here $\sigma_{i}$ is the measured differential cross section for data point $i$, $\sigma\left(q_{i}\right)$ is the differential cross section as calculated from eqn.(169) for data point 1 , and $\varepsilon_{i}$ is the error in the measured differential cross section. As the parameters are varied and $x^{2}$ reaches its minimum value, the optimum values for the varying parameters $n_{k}$ are obtained.

As an indication of how well eqn. (169) describes the data, we make use of the value of reduced $x^{2}$

$$
x_{R}^{2}=x^{2} / D F
$$

where $D F$ is the number of degrees of freedom in the $\mathrm{fit}$ and is equal to the number of data points $i$ less the number of parameters $k$ allowed to vary in the fit. From the value of $x_{R}^{2}$ obtained from the $f i t$ and the number of degrees of freedom, there is a corresponding 
probability $P\left(X^{2}, D F\right)$ that a random sample of data points vould give a value of $x^{2}$ equal to or larger than that obtained. A good fit vould nake this probabllity 50\%, which mears that we vould expect that half the time a random sample of data points vould give higher values of $x^{2}$ and half the time a sample vould give a lower value. For most cases $X_{R}^{2}=1$ corresponds to a $50 \%$ probabllity of exceeding $x^{2}$, that is the function $P\left(X^{2}, D F\right)$ has a value of 0.5 at $x_{R}^{2} \approx 1$.

The search for the minimum value of $x^{2}$ can be viewed as searching a $k$ dimensional parameter space for the minimum of $x^{2}$ which is a continuous function of the $k$ parameters. There are a number of ways that one can search through parameter space looking for the minimum of $x^{2}$ and we employ a combination of two commonly used methods ${ }^{19}$. In order to approach the minimum fairly rapidly, ve start the search using the gradient search method. Then when the search has been brought to the general neighborhood of the minimum, the grid search method is used to locate the minimum more precisely.

The gradient search is a good way to start the search for the minimum because it searches in the direction of maximum variation of $x^{2}$, thus insuring rapid travel in the general direction of the minimum. This is accomplished by calculating the gradient of $x^{2}$ in parameter space and varying all the parameters simultaneously such that the direction of the search is along the gradient or the direction of steepest descent of $x^{2}$. In the neighborhood of the minimum the gradient search method is not expedient to use because the gradient vanishes at the minimum. At this point in the search it is therefore better to use a different method to get to the actual 
minimum. The method we choose is the giid search method in which $x^{2}$ is minimized with respect to each parameter separately. Locating the minimum for each parameter in turn and repeating this procedure produces $x^{2}$ which is minimized with respect to all the parameters.

There are a number of different ways of fitting the data to the parameterized formula of eqn.(169). Some parameters can be fixed while letting others vary. Giving some parameters fixed values increases the sensitivity of the varying parameters to the fit. Since the spin-orbit part of the amplitude plays a minor role at small angles, the fit will be insensitive to the parameters associated with the spin-orbit interaction. Thus these parameters $\left(r_{1}, r_{2}\right.$, and in general $\left.b_{3}\right)$ will not be allowed to vary. In addition, one can introduce other parameters which correct for normalization and of fset in scattering angle. Introducing parameters $N$ and $\Delta q$ to take into account these corrections, the parameterized differential cross section we will use has the form

$$
\frac{d \sigma}{d \Omega}=N \frac{d \sigma}{d \Omega}(q+\Delta q)
$$

Table 8 lists some of the different ways that the data can be fitted to the parameterized differential cross section. The results of the fits for the lead, calcium, and carbon data are tabulated in tables 9,10 , and 11 respectively. Only the data for the spectrometer angle settings of $-0.83^{\circ}$ and $2.5^{\circ}$ were used in the $f i t$. For the lead data, the first diffraction minimum is close to the 
Table 8 - Variants used to f1t the data to Eqn.(169).

\begin{tabular}{|c|c|}
\hline Vartant & Description \\
\hline$A$ & Vary: $a, b_{1}=b_{3}, \sigma_{t o t}$ Fix: $r_{1}=r_{2}=10 f m$ \\
\hline B & Vary: $\alpha, b_{1}=b_{3}, \sigma_{\text {tot }}, \Delta q, N_{1}, N_{2} F 1 x: r_{1}=r_{2}=10 f m$ \\
\hline C & Vary: $a, b_{1}=b_{3}, \sigma_{t o t}, N_{1}, N_{2}$ Pix: $r_{1}=r_{2}=10 \mathrm{fm}$ \\
\hline D & Vary: $\alpha, b_{1}=b_{3}, \sigma_{t o t}, N$ Fix: $r_{1}=r_{2}=10 \mathrm{fm}$ \\
\hline $\mathbf{E}$ & Vary: $a_{1}, b_{1}=b_{3}, \sigma_{t o t}, \Delta q$ Fix: $r_{1}=r_{2}=10 \mathrm{fm}$ \\
\hline $\mathbf{F}$ & Vary: $a, b_{1}=b_{3}, \sigma_{\text {tot }}, \Delta q, N$ Fix: $r_{1}=r_{2}=10 \mathrm{fm}$ \\
\hline G & Vary: $a, b_{1}=b_{3}, \sigma_{t o t}, \Delta q, N$ Fix: $r_{1}=r_{2}=10 \mathrm{fm} u=1$ \\
\hline $\mathbf{H}$ & Vary: $\alpha, b_{1}=b_{3}, \sigma_{t o t}, \Delta q, N$ Fix: $r_{1}=r_{2}=10 f m \phi=\phi^{\prime}$ \\
\hline I & Vary: $\alpha, b_{1}, b_{3}, \sigma_{\text {tot }} F i x: r_{1}=r_{2}=10 \mathrm{fm}$ \\
\hline J & Vary: $\alpha, b_{1}, \sigma_{\text {tot }}, N$ Fix: $r_{1}=r_{2}=10 \mathrm{fm} b_{3}=100$ \\
\hline $\mathbf{K}$ & Vary: $\alpha, b_{1}, \sigma_{\text {tot }}, N F i x: r_{1}=r_{2}=10 \mathrm{fm} b_{3}=10000000$. \\
\hline$L$ & Vary: $a, b_{1}, \sigma_{\text {tot }}, N F 1 x: r_{1}=r_{2}=0$ \\
\hline
\end{tabular}

Coulamb-nuclear interference region and since the parameterization does not describe diffraction minima, only the first 14 data points of the lead data vere used in the fit. For variants $B$ and $C$ in table 8, two values for the normalization parameters $N$ vere used, $N_{1}$ and $N_{2}$. $N_{1}$ was used for the $-0.83^{\circ}$ data and $N_{2}$ for the $2.5^{\circ}$ data. This was done only because the relative beam intensity vas measured vith two different devices for the two spectrometer angle settings and a discrepancy between $N_{1}$ and $N_{2}$ might prove to be interesting. Variant $G$ uses a value of 1 for $V$ in eqn. (169), and variant $H$ uses another common form' for the phase $\phi$ 


$$
\varphi=\varphi^{\prime}=\frac{\beta}{2} \ln (0.209 / q)
$$

The fitted values of $b_{3}$ for variant $I$ which are not listed in the tables are $-119.0 \pm 4117.3,83.8 \pm 1545.9,-269.2 \pm 64.2$ for the lead, calcium, and carbon data respectively. The fact that the errors for $b_{3}$ are so large when $1 t$ is allowed to vary means that this parameter has such a negligible role in the parameterized differential cross section that it could be neglected. Indeed, when the spin-orbit contributions are turned off by setting $r_{1}$ and $r_{2}$ equal to zero, the effect on the fit is extremely small as seen by comparing the results of variants $J, K$, and $L$ for the three sets of data.

In figures 46,47 , and 48 the differential cross sections for the three targets are plotted as a function of four-momentum transfer squared. This allows the contribution of the exponential decrease of the cross section with $q^{2}$ to be seen. Also shown for the calcium and lead targets are theoretical calculations for different aspects of the scattering process. The short dashed line is the pure nuclear contribution from a relativistic impulse approximation calculation (to be discussed in the next section), and the long dashed line represents pure Coulomb scattering, or the relativistic Rutherford cross section. The solid line is the full theoretical prediction of nuclear and Coulomb scattering with interference between the two included. For carbon, only the Coulomb cross section is shown as no nuclear theoretical calculation exists at this time for this nucleus. 
Table 9 - Results of fits of lead data to variants of table 8 .

\begin{tabular}{|c|c|c|c|c|c|c|c|}
\hline Variant & $x_{\mathrm{R}}^{2}$ & $\alpha$ & $b_{1}$ & $\sigma_{\text {tot }}$ & $N_{1}$ & $\mathrm{~N}_{2}$ & $\Delta q$ \\
\hline \multirow[t]{2}{*}{$A$} & 5.20 & 0.724 & 471.7 & 3476.5 & & & \\
\hline & & \pm 0.038 & \pm 5.51 & \pm 30.32 & & & \\
\hline \multirow[t]{2}{*}{$\mathrm{B}$} & 0.49 & -0.061 & 501.3 & 3726.9 & 0.76 & 0.81 & -0.42 \\
\hline & & \pm 0.009 & \pm 6.90 & \pm 39.97 & \pm 0.009 & \pm 0.004 & \pm 0.07 \\
\hline \multirow[t]{2}{*}{$C$} & 0.71 & -0.043 & 478.1 & 3598.3 & 0.80 & 0.80 & \\
\hline & & \pm 0.009 & \pm 7.05 & \pm 39.50 & \pm 0.009 & \pm 0.004 & \\
\hline \multirow[t]{2}{*}{ D } & 0.67 & -0.078 & 481.5 & 3558.1 & 0.78 & & \\
\hline & & \pm 0.009 & \pm 6.39 & \pm 39.44 & \pm 0.004 & & \\
\hline \multirow[t]{2}{*}{$E$} & 3.38 & 0.85 & 452.2 & 3190.7 & & & 0.52 \\
\hline & & \pm 0.036 & \pm 5.69 & \pm 17.31 & & & \pm 0.02 \\
\hline \multirow[t]{2}{*}{$F$} & 0.62 & -0.083 & 483.5 & 3594.0 & 0.78 & & -0.08 \\
\hline & & \pm 0.009 & \pm 6.84 & \pm 39.10 & \pm 0.004 & & \pm 0.04 \\
\hline \multirow[t]{2}{*}{ G } & 0.58 & -0.075 & 495.0 & 3611.9 & 0.79 & & -0.08 \\
\hline & & \pm 0.009 & \pm 6.84 & \pm 39.07 & \pm 0.004 & & \pm 0.05 \\
\hline \multirow[t]{2}{*}{$\mathrm{H}$} & 1.26 & 0.111 & 418.9 & 3404.4 & 0.58 & & -0.12 \\
\hline & & \pm 0.012 & \pm 5.67 & \pm 28.28 & \pm 0.003 & & \pm 0.07 \\
\hline \multirow[t]{2}{*}{ I } & 5.70 & 0.795 & 461.7 & 3375.5 & & & \\
\hline & & \pm 0.034 & \pm 5.38 & \pm 28.16 & & & \\
\hline \multirow[t]{2}{*}{$\mathrm{J}$} & 0.68 & -0.022 & 476.0 & 3594.9 & 0.80 & & \\
\hline & & \pm 0.010 & \pm 7.06 & \pm 39.68 & \pm 0.004 & & \\
\hline \multirow[t]{2}{*}{ K } & 0.68 & -0.022 & 476.2 & 3594.9 & 0.80 & & \\
\hline & & \pm 0.010 & \pm 7.00 & \pm 39.64 & \pm 0.004 & & \\
\hline \multirow[t]{2}{*}{$L$} & 0.68 & -0.022 & 476.0 & 3594.9 & 0.80 & & \\
\hline & & \pm 0.010 & \pm 7.06 & \pm 39.68 & \pm 0.004 & & \\
\hline
\end{tabular}


Table 10 - Results of fits of calcium data to variants of table 8 .

\begin{tabular}{|c|c|c|c|c|c|c|c|}
\hline Variant & $x_{\mathrm{R}}^{2}$ & $\alpha$ & $\mathrm{b}_{1}$ & $\sigma_{t o t}$ & $N_{1}$ & $\mathrm{~N}_{2}$ & $\Delta q$ \\
\hline \multirow[t]{2}{*}{ A } & 0.97 & 0.108 & 139.2 & 973.2 & & & \\
\hline & & \pm 0.011 & \pm 1.56 & \pm 3.62 & & & \\
\hline \multirow[t]{2}{*}{ B } & 0.85 & 0.047 & 137.6 & 969.1 & 0.94 & 0.99 & -0.37 \\
\hline & & \pm 0.010 & \pm 1.55 & \pm 3.61 & \pm 0.007 & \pm 0.006 & \pm 0.07 \\
\hline \multirow[t]{2}{*}{$\mathrm{C}$} & 1.10 & 0.070 & 135.8 & 963.3 & 0.98 & 0.99 & \\
\hline & & \pm 0.010 & \pm 1.80 & \pm 3.61 & \pm 0.008 & \pm 0.006 & \\
\hline \multirow[t]{2}{*}{$D$} & 1.18 & 0.055 & 134.6 & 969.9 & 0.97 & & \\
\hline & & \pm 0.010 & \pm 1.67 & \pm 3.80 & \pm 0.005 & & \\
\hline \multirow[t]{2}{*}{$\mathrm{E}$} & 1.05 & 0.108 & 139.2 & 973.2 & & & 0.00 \\
\hline & & \pm 0.011 & \pm 1.56 & \pm 3.61 & & & \pm 0.07 \\
\hline \multirow[t]{2}{*}{$F$} & 1.02 & 0.031 & 135.3 & 981.8 & 0.95 & & -0.10 \\
\hline & & \pm 0.010 & \pm 1.68 & \pm 3.91 & \pm 0.005 & & \pm 0.07 \\
\hline \multirow[t]{2}{*}{ G } & 1.05 & 0.102 & 140.2 & 987.8 & 0.98 & & -0.06 \\
\hline & & \pm 0.011 & \pm 1.65 & \pm 3.83 & \pm 0.005 & & \pm 0.07 \\
\hline \multirow[t]{2}{*}{11} & 1.60 & 0.090 & 126.8 & 985.6 & 0.87 & & 0.03 \\
\hline & & \pm 0.012 & \pm 1.68 & \pm 3.84 & \pm 0.004 & & \pm 0.09 \\
\hline \multirow[t]{2}{*}{$I$} & 1.04 & 0.102 & 137.9 & 968.6 & & & \\
\hline & & \pm 0.011 & \pm 1.56 & \pm 3.61 & & & \\
\hline \multirow[t]{2}{*}{$\mathrm{J}$} & 1.21 & 0.046 & 134.6 & 967.76 & 0.97 & & \\
\hline & & \pm 0.010 & \pm 1.58 & \pm 3.63 & \pm 0.004 & & \\
\hline \multirow[t]{2}{*}{$\mathrm{K}$} & 1.20 & 0.046 & 134.4 & 967.8 & 0.97 & & \\
\hline & & \pm 0.010 & \pm 1.49 & \pm 3.62 & \pm 0.004 & & \\
\hline \multirow[t]{2}{*}{$L$} & 1.20 & 0.046 & 134.4 & 967.8 & 0.97 & & \\
\hline & & \pm 0.010 & \pm 1.49 & \pm 3.62 & \pm 0.004 & & \\
\hline
\end{tabular}


Table 11 - Results of fits of carbon data to variants of table $B$.

\begin{tabular}{|c|c|c|c|c|c|c|c|}
\hline Varlant & $x_{\mathrm{R}}^{2}$ & $\alpha$ & $b_{1}$ & $\sigma_{\text {tot }}$ & $\mathbf{N}_{1}$ & $\mathbf{N}_{2}$ & $\Delta q$ \\
\hline \multirow[t]{2}{*}{$A$} & 7.20 & 0.222 & 81.9 & 378.5 & & & \\
\hline & & \pm 0.007 & \pm 1.43 & \pm 1.21 & & & \\
\hline \multirow[t]{2}{*}{ B } & 0.61 & -0.449 & 45.5 & 277.1 & 1.19 & 1.23 & -0.57 \\
\hline & & \pm 0.007 & \pm 1.47 & \pm 0.86 & \pm 0.011 & \pm 0.007 & \pm 0.09 \\
\hline \multirow[t]{2}{*}{$\mathrm{C}$} & 0.87 & -0.437 & 45.2 & 282.1 & 1.21 & 1.19 & \\
\hline & & \pm 0.006 & \pm 1.49 & \pm 0.98 & \pm 0.013 & \pm 0.007 & \\
\hline \multirow[t]{2}{*}{ D } & 0.98 & -0.252 & 57.7 & 304.0 & 1.29 & & \\
\hline & & \pm 0.007 & \pm 1.48 & \pm 1.03 & \pm 0.006 & & \\
\hline \multirow[t]{2}{*}{$\mathbf{E}$} & 1.62 & 0.726 & 49.3 & 272.0 & & & 0.59 \\
\hline & & \pm 0.006 & \pm 1.48 & \pm 0.93 & & & \pm 0.13 \\
\hline \multirow[t]{2}{*}{$\mathbf{F}$} & 0.56 & -0.269 & 54.2 & 298.4 & 1.29 & & -0.31 \\
\hline & & \pm 0.007 & \pm 1.46 & \pm 1.01 & \pm 0.006 & & \pm 0.09 \\
\hline \multirow[t]{2}{*}{ G } & 0.56 & -0.271 & 53.6 & 297.3 & 1.30 & & -0.30 \\
\hline & & \pm 0.007 & \pm 1.47 & \pm 1.02 & \pm 0.006 & & \pm 0.09 \\
\hline \multirow[t]{2}{*}{$\mathbf{H}$} & 0.79 & 0.278 & 40.7 & 284.9 & 1.30 & & -0.18 \\
\hline & & \pm 0.007 & \pm 1.68 & \pm 1.02 & \pm 0.006 & & \pm 0.09 \\
\hline \multirow[t]{2}{*}{ I } & 2.58 & -0.725 & 54.7 & 272.6 & & & \\
\hline & & \pm 0.006 & \pm 1.53 & \pm 0.93 & & & \\
\hline \multirow[t]{2}{*}{$\mathrm{J}$} & 1.23 & -0.294 & 55.2 & 299.2 & 1.26 & & \\
\hline & & \pm 0.006 & \pm 1.49 & \pm 1.01 & \pm 0.006 & & \\
\hline \multirow[t]{2}{*}{$\mathbf{K}$} & 1.03 & -0.298 & 50.8 & 291.6 & 1.29 & & \\
\hline & & \pm 0.006 & \pm 1.48 & \pm 0.98 & \pm 0.006 & & \\
\hline \multirow[t]{2}{*}{$L$} & 1.03 & -0.297 & 50.8 & 291.6 & 1.29 & & \\
\hline & & \pm 0.006 & \pm 1.48 & \pm 0.99 & \pm 0.006 & & \\
\hline
\end{tabular}




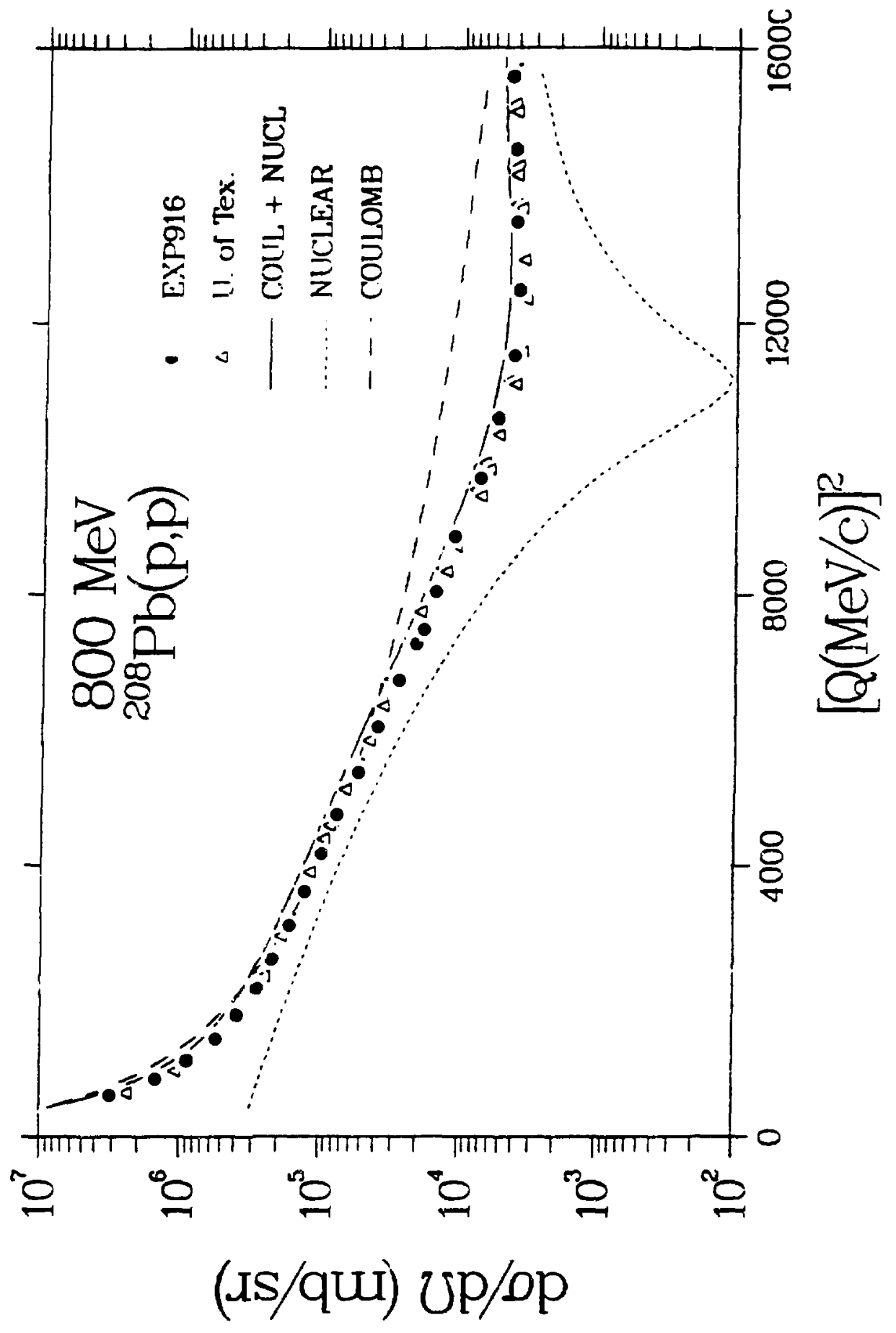

P1g. 46

Differential Cross section as a function

of $q^{2}$ for lead target. 


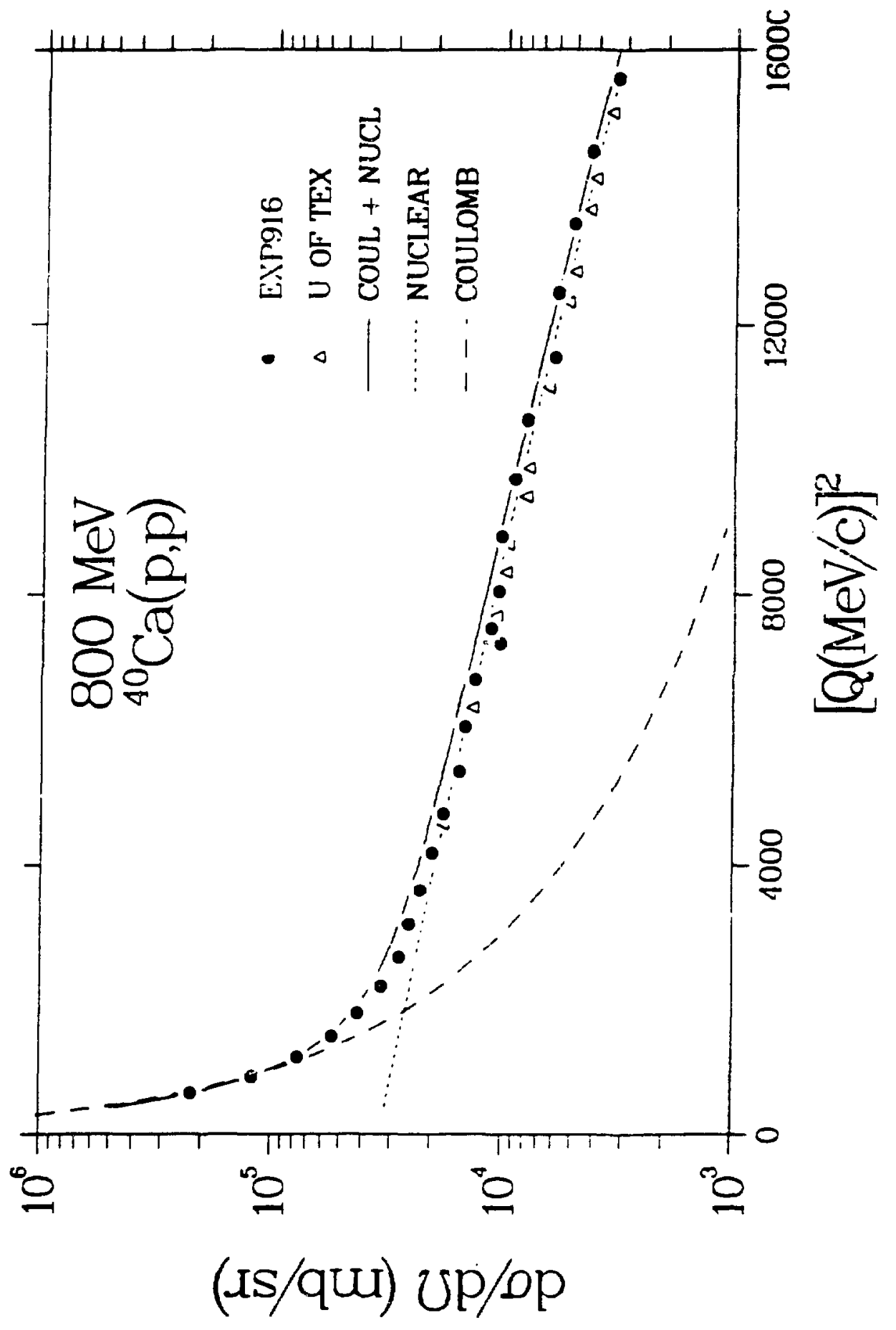

P15. 47

Differential Cross section as a function

of $q^{2}$ for calciun target. 


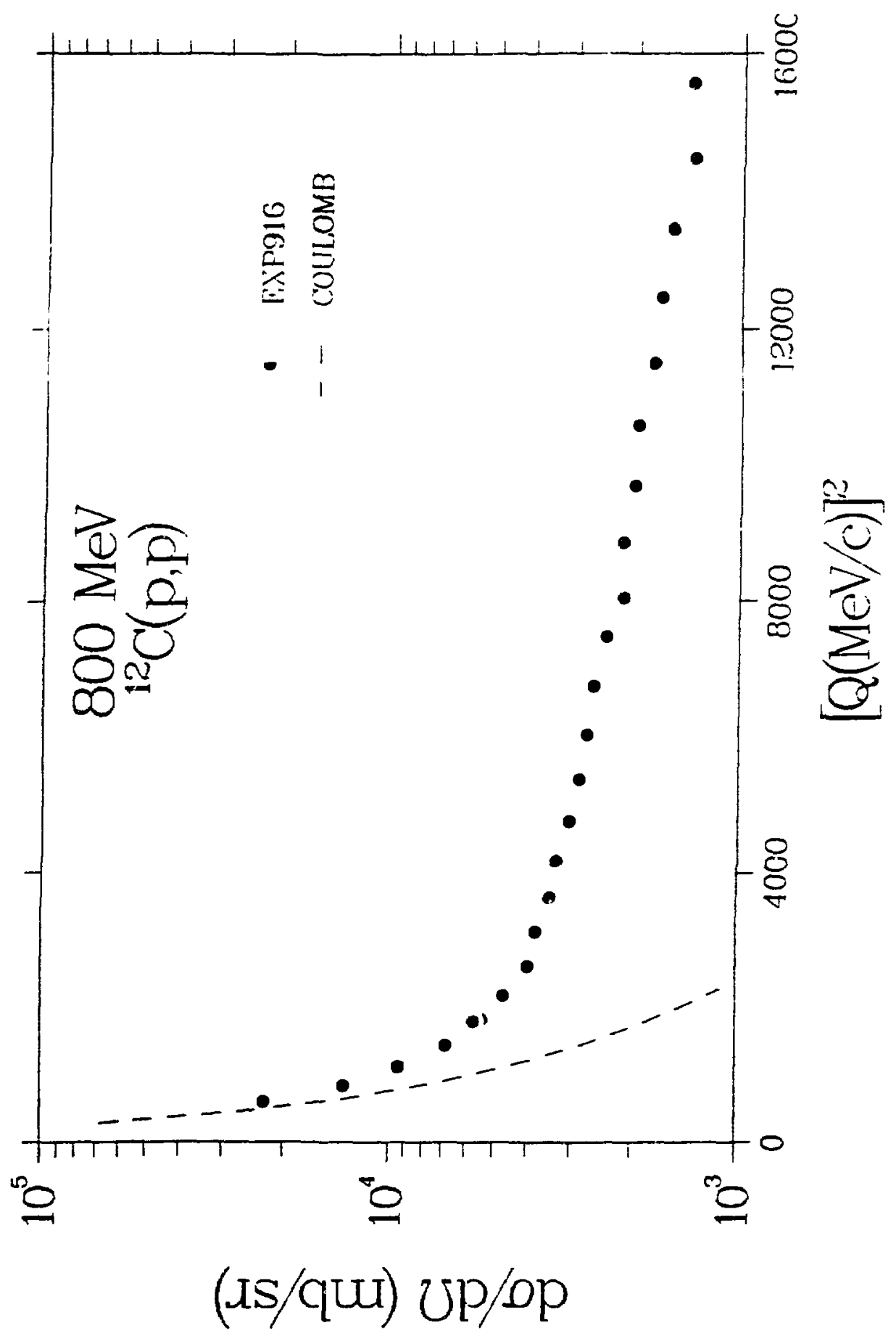

Fig. 48

Differential Cross section as a function of $\mathbf{q}^{2}$ for carbon target. 


\section{V-B. PHYSICS OBTAINABLE FROM SMALL ANGLE SCATTERING}

There are several theories describing the scattering of nucleons off of nuclei. These theories can be categorized into two types: Relativistic (R) and Non-relativistic (NR). The difference between these two classes is not just of kinematical origin. There are distinct dynamical differences between non-relativistic and relativistic scattering descriptions. In fact, the phrase "non-relativistic scattering theories" is taken to mean theories using relativistic kinematics but non-relativistic dynamics. Since each class predicts different results for measurable quantities, data that will focus in on the differences between these theories is highly desirable. It is only through high quality data that these theories can be tested. Perhaps it will then be possible to understand what is happening at the microscopic level of elastic scattering.

An example of a tool that can be used to discern between NR and $R$ theories is the extraction from Coulomb-Nuclear interference the value of the nuclear phase $\cot ^{-1} \alpha$ at $q=0$. Figure 49 shows the behaviour of $\alpha$ described by the Non-relativistic and Relativistic Impulse Approximations (NRIA and RIA) as a function of incident proton energy'. The behaviour shown for protons incident on ${ }^{4}$ He is also descriptive for the nuclear targets of this work. A conclusion made from this figure is that, for the 800 MeV data presented in this work, it is impossible to use the obtained value of a to make any conclusions about the two theoretical descriptions. However, the 


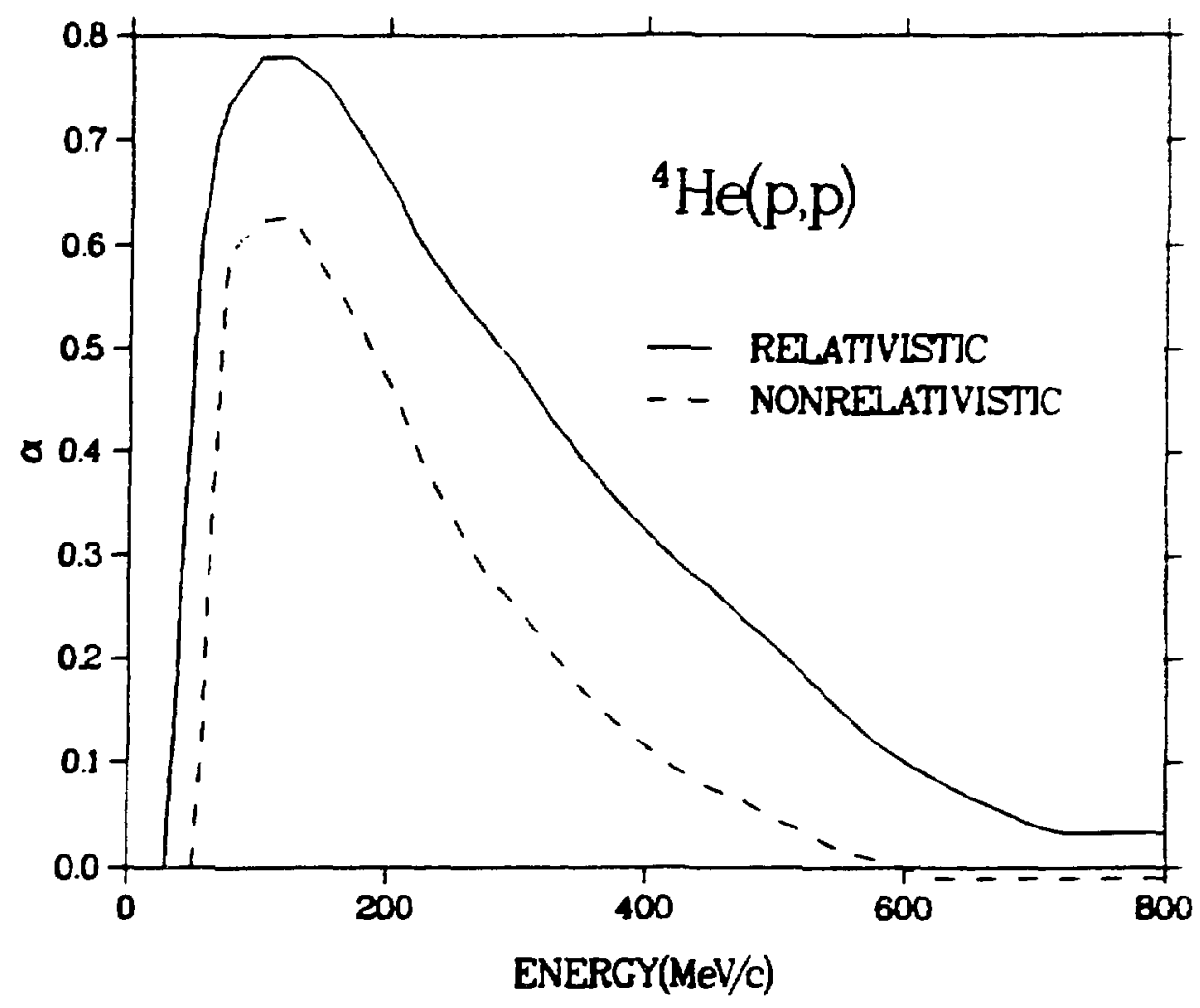

Fig. 49

Dependence of $\alpha$ at $q=0$ on beam energy.

figure also shows that having $\alpha$ from lower energy differential cross section data would provide highly valuable information.

In order to better understand the current problems in dealing with the physical interpretations of modern scattering theories, it is useful to discuss the progression of theory in describing data over the past 20-30 years ${ }^{23}$. The non-relativistic approach vas the first to emerge with the multiple scattering theory of Kerman, McManus, and Thaler (KMT) in 195924. This theory was successful in describing p-Nucleus elastic differential cross section data at $1 \mathrm{GeV}$ for a variety of nuclei. Because of its success, it was widely 
thought that non-relativistic analyses of elastic scattering data could be used to extract nuclear structure irformation. The non-relativistic formalism was generally accepted as fact, a tool to be used to probe the interior of nuclei. This was the attitude of many physicists in the 1970's.

As more data became available, doubt began to set in with regard to the non-relativistic theory being a fact of life. KMT impulse approximation descriptions of $800 \mathrm{MeV}$ p-Nucleus elastic analyzing power data were not that good. At first this problem was blamed on the lack of knowledge about the nucleon-nucleon amplitudes, particularly the proton-neutron amplitude, since experimental data did not adequately constrain the phase shift solutions. It was therefore decided to obtain data at energies less than $800 \mathrm{MeV}$ where phase shift solutions gave well constrained pN amplitudes for use in the non-relativistic calculations and yet at high enough energies where the impulse approximation was still valid. 400 and $500 \mathrm{MeV}$ were chosen as the energies at which to obtain data in order to test the non-relativistic theory.

Differential cross section and analyzing power data for p-Nucleus elastic scattering at 400 and $500 \mathrm{MeV}$ verified that a serious problem existed with the KHT impulse approximation 25,26 . And when it became possible to measure the spin rotation parameter 0 , defined in the language of section II-C as 


$$
0=\frac{2 \operatorname{Im}\left(\alpha_{n}\left(i \gamma_{n}\right)^{\star}\right)}{\left(\frac{d \sigma}{d \Omega}\right)_{U N}}
$$

it too was found not to be well described by KMT at $500 \mathrm{MeV}^{23}$. At this point, around 1980, the first relativistic formalism for describing p-Nucleus elastic scattering came forth. This was a phenomenological optical model based on the Dirac equation ${ }^{2}$.

The success of the phenomenological Dirac optical potential model to describe p-Nucleus elastic scattering data was quite extraordinary. This model was based on using twelve fitting parameters to fit the p-Nucleus data. The twelve parameters come from three-parameter Wood-Saxon potentials used for each of the real and imaginary scalar and vector potentials as input to the Dirac equation

$$
(p-m-\dot{U}) \Psi=0, \quad \dot{U}=s+\gamma^{0} \mathrm{~V}
$$

Because Dirac phenomenology was so successful in correctly predicting 500 MeV spin observables and the KMT impulse approximation failed at this energy, it was suggested that the dynamics inherent in the relativistic formalism that were absent in the non-relativistic description must be present in the scattering prccess. Specifically, virtual $N \bar{N}$ pair production was seen as the key ingredient present in the Dirac approach that was responsible for its success.

Virtual $N \bar{N}$ pair processes enter into the description of 
relativistic p-Nucleus elastic scattering because of the presence of positive $\psi^{+}$and negative $\psi^{-}$components of the Dirac wave function ${ }^{29}$. $\psi^{+}$components describe forward-in-time propagation of positive energy nucleon states and $\psi^{-}$components describe backward-in-time propagation of negative energy nucleon states. But $\psi^{-}$components can also be interpreted to describe forward-in-time propagation of positive energy antinucleon states. Thus a scattering process such as that 1llustrated in fig. 50 may occur. This diagram is what is commonly called a $z$-graph for obvious reasons. It describes the propagation of the incident nucleon with momentum $\overrightarrow{\vec{g}}$ which interacts

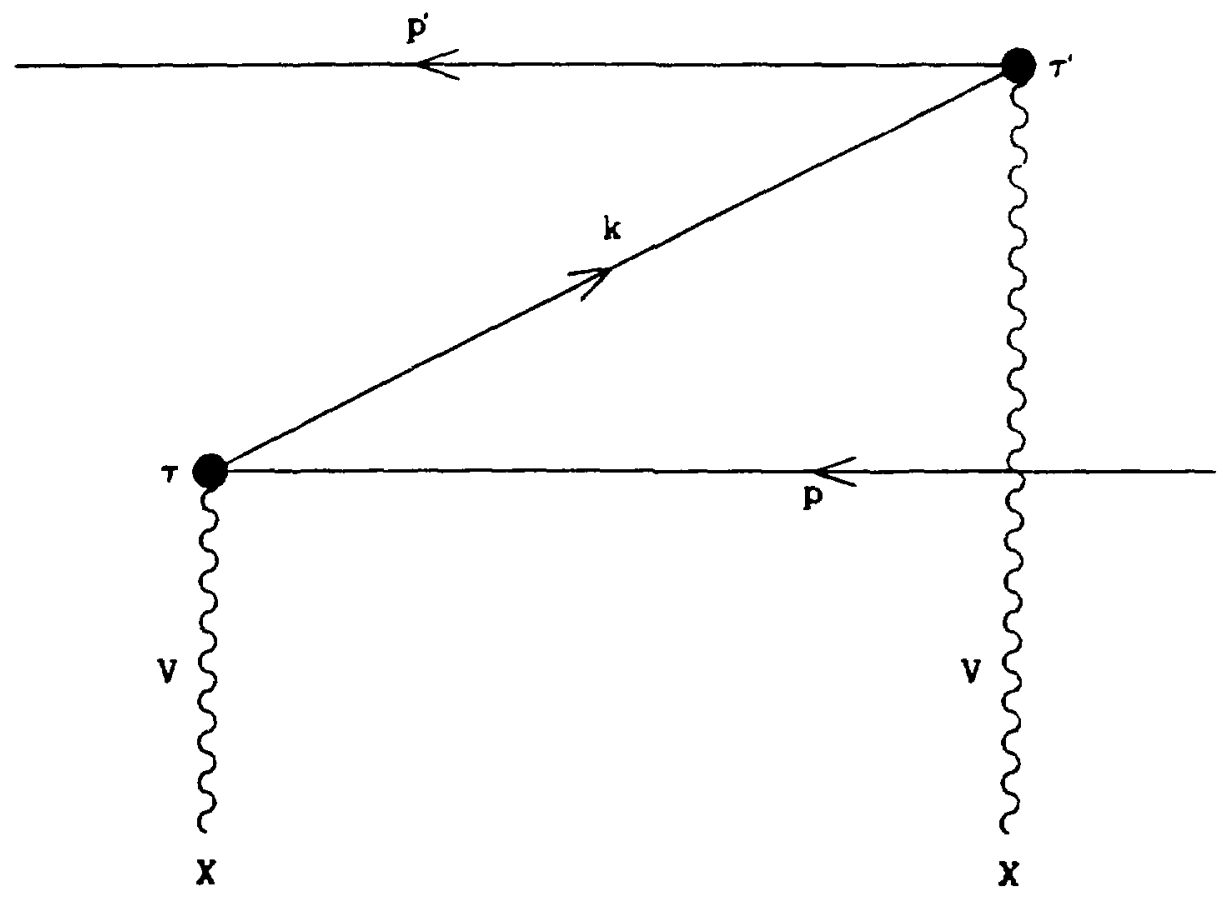

Fig. 50

2-graph description of relativistic scattering. 
with the nucleus instantaneously at tine $\tau$. The incident nucleon annihilates with an antinucleon that vas created instantaneousiy at an earlier time $\tau^{\prime}$. The observed final state of the projectile propagates vith momentum $\vec{p}^{\prime}$ from the point of production of the antinucleon. This production of virtual NÑ pair is absent in the non-relativistic formalism. Figure 51 shows the non-relativistic graph corresponding to the relativistic 2 -graph of figure 50 . It vould therefore seem that if one vere to isolate the factor contributing to virtual Nī pair processes in the Dirac equation and then neglect it, the non-relativistic description vould result. This is indeed what happens ${ }^{30}$. If one vrites the Dirac equation as two coupled equations relating the coupling of the positive and negative energy states as given by

$$
\begin{aligned}
& \left(E-E_{p}-U^{++}\right) \psi^{+}=U^{+-} \psi^{-} \\
& \left(E+E_{p}-U^{--}\right) \psi^{-}=U^{-+} \psi^{+}
\end{aligned}
$$

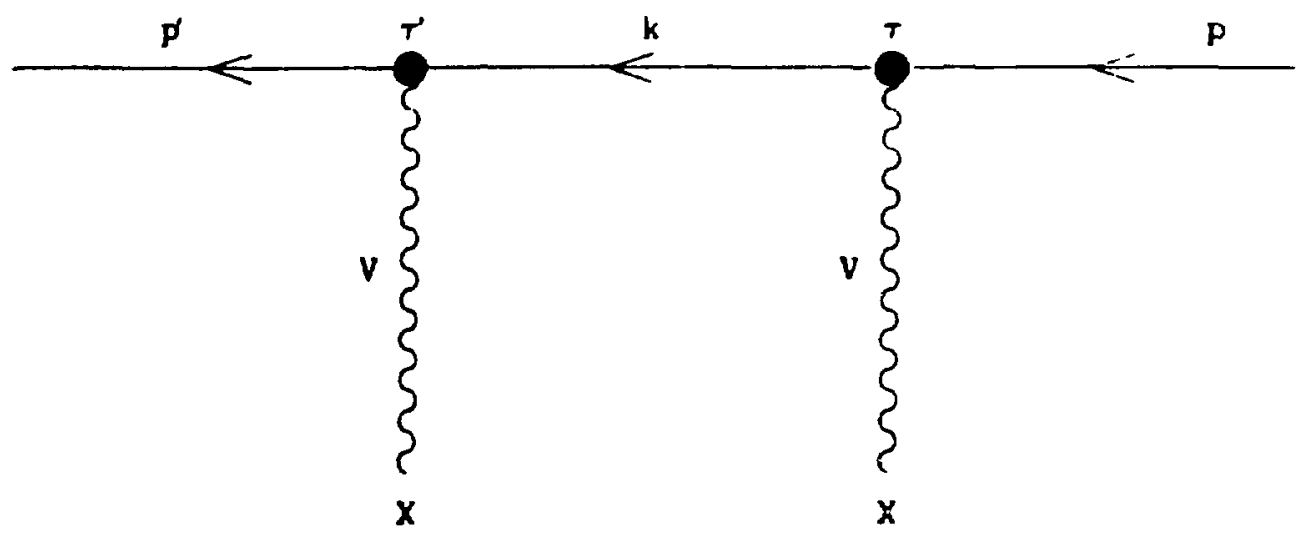

Pig. 51

Graph describing non-relativistic scattering. 
and then eliminates the components, the result can be expressed as

$$
\left(E-E_{p}-U^{++}-U_{p a i r}\right) \psi^{+}=0
$$

where $\mathrm{U}^{++}, \mathrm{U}^{+-}, \mathrm{U}^{-+}$, and $\mathrm{U}^{--}$are given by

$$
U^{P^{\prime}} P\left(\vec{p}^{\prime}, \vec{p}\right)=\vec{u} p^{\prime}\left(\vec{p}^{\prime}\right) \dot{U}\left(\vec{p}^{\prime}, \vec{p}\right) u^{P}(\vec{p})
$$

$\dot{U}$ is the Dirac optical potential, ${u^{+}}^{+}$and $u^{-}$are the positive and negative energy free particle Dirac spinors, and $U_{p a i r}$ is the potential associated with virtual $N \bar{N}$ pair processes. It is given by

$$
U_{p a i r}=U^{+-} \frac{1}{E+E_{p}-U^{--}} U^{-+}
$$

Setting $v_{\text {pair }}$ equal to zero in eqn. (180) results in basically the Schrobinger equation witi relativistic kinematics.

With the success of Dirac phenomenology, Dirac equation based impulse approximation vas developed in order to be able to describe the data in a relativistic way that is parameter free ${ }^{32}$. This impulse epproximation vas founded on the first order Born approxination for the scattering amplitude

$$
f_{s^{\prime} s^{\prime}}\left(\vec{p}^{\prime}, \vec{p}\right)=-\frac{2}{2 \pi} \bar{u}_{s^{\prime}}^{+}\left(\vec{p}^{\prime}\right) \dot{u}\left(\vec{p}^{\prime}, \vec{p}\right) u_{s}^{+}(\vec{p})
$$

Here the $s$ and $s^{\prime}$ refer to the initial and final spin states of the 
profectile. The inpulse approximation for the scattering amplitude is given by

$$
\begin{aligned}
& f_{s^{\prime} s^{\prime}}\left(\vec{p}^{\prime}, \vec{p}\right)=2 \operatorname{ip} \int \frac{d^{3} k}{(2 \pi)^{3}} \sum_{\alpha} \bar{u}_{s^{\prime}}^{+}\left(\vec{p}^{\prime}\right) \bar{\psi}_{\alpha}\left(\vec{k}+\frac{2}{2} \vec{q}\right) \dot{F}\left(\vec{p}, \vec{p}^{\prime}, \vec{k}\right) x \\
& \psi_{\alpha}\left(\vec{k}-\frac{1}{2} \vec{q}\right) u_{s}^{+}(\vec{p})
\end{aligned}
$$

Here $\Psi_{\alpha}(\vec{R})$ is a nucleon bound state vave function with quantum numbers $\alpha$ and eigenenergtes $c_{\alpha}=\left(m^{2}+\vec{k}^{2}\right)^{1 / 2}$ and $\dot{F}$ is a Lorentz invariant NN amplitude. One can see by comparison of eqns. and (184) that the Dirac optlcal potential in the full Dirac space of two nucleons will be

$$
\dot{u}\left(\vec{p}^{\prime}, \vec{p}\right)=-\frac{4 \pi 1 p}{m} \int \frac{d^{3} k}{(2 \pi)^{3}} \sum_{\alpha} \bar{\psi}_{\alpha}\left(\vec{k}+\frac{1}{2} \vec{q}\right) \dot{F}\left(\vec{p}, \vec{p}^{\prime}, \vec{k}\right) \psi_{\alpha}\left(\vec{k}-\frac{2}{2} \vec{q}\right)
$$

This can be rewritten as

$$
\dot{U}\left(\vec{p}^{\prime}, \vec{p}\right)=-\frac{1}{4} \operatorname{Tr}_{2}\left[\int \frac{d^{3} k}{(2 \pi)^{3}} \dot{H}\left(\vec{p}, \vec{p}^{\prime}, \vec{k}\right) \dot{p}(\vec{k}, \vec{q})\right]
$$

where $\dot{M}$ is the Feynman amplitude

$$
\dot{M}\left(\vec{p}, \vec{p}^{\prime}, \vec{k}\right)=\frac{4 \pi \dot{p} \dot{p}}{m} \dot{F}\left(\vec{p}, \vec{p}^{\prime}, \vec{k}\right)
$$


The trace in eqn. (186) is over the Dirac indices of particle 2 . The relativistic $4 \times 4$ density matrix is defined as

$$
\dot{p}(\vec{k}, \vec{q})=4 \sum_{\alpha} \psi_{\alpha}\left(\vec{k}-\frac{1}{2} \vec{q}\right) \bar{\psi}_{\alpha}\left(\vec{k}+\frac{1}{2} \vec{q}\right)
$$

Note the similarity between the Dirac optical potential given by eqn. (186) and the non-relativistic "to" optical potential given by

$$
\bar{j}_{N R}\left(\vec{p}^{\prime}, \vec{p}\right)=\frac{1}{2} \operatorname{Tr}_{2}\left[\int \frac{d^{3} k}{(2 \pi)^{3}} t\left(\vec{p}, \vec{p}^{\prime}, \vec{k}\right) \rho(\vec{k}, \vec{q})\right]
$$

with the non-relativistic $t$-matrix and $\rho$ the $2 \times 2$ nuclear density matrix in Pauli space.

It is common to factorize the Feynman amplitude $\dot{M}$ from out of the integral in eqn. (186), evaluating it a $R=0$. This is done based on the assumption that $\dot{p}$ varies much more rapidly with $R$ than does the NN amplitude $\dot{F}$. The Dirac optical potential will then be

$$
\dot{U}\left(\vec{p}^{\prime}, \vec{p}\right)=-\frac{1}{4} \operatorname{Tr}_{2}\left[\dot{M}\left(\vec{p}, \vec{p}^{\prime}\right) \dot{\rho}(\vec{q})\right]
$$

with

$$
\dot{p}(\vec{q})=\int \frac{d^{3} k}{(2 \pi)^{3}} \dot{p}(\vec{k}, \vec{q})
$$

Thus in order to construct the Dirac optical potential, one requires two ingredients: 1) the Feynman amplitude for NN scattering, and 2) 
the relativistic nuclear density matrix. The relativistic nuclear density matrix is determined from Dirac-Hartree wave functions as input to eqn. (188). There are, however, a variety of forms fossible for $\dot{M}$. And it is on finding a suitable form for $\dot{M}$ that attention has been focused in recent years.

The first form for $\dot{M}$, or equivalently $\dot{F}$, is what has come to be known as $I A I^{32}$. In this form of the Impulse approximation, the Locentz invariant $N N$ amplitude is expanded in terms of 5 fermi covariants:

$$
\dot{F}=\sum_{i=1}^{5} F_{i}(s, t, u) K_{i}
$$

The $K_{1}$ are given in table 12. $F_{1}$ is thus a scalar amplitude, $F_{2}$ a vector amplitude, etc. The arguments of the $F_{i}, s, t$, and $u$, are the Mandelstam variables. It is natural to associate the $F_{i}$ with meson

Table 12 - Fermi Covariants used in IAl.

i

$k_{i}$

$\begin{array}{ll}\text { 1(scalar) } & 1 \\ 2 \text { (vector) } & r_{1} \cdot r_{2} \\ 3 \text { (pseudoscalar) } & r_{1}^{5} r_{2}^{5} \\ 4 \text { (tensor) } & \sigma_{1}^{\nu} \sigma_{2 \mu \nu} \\ 5 \text { (axialvector) } & r_{1}^{5} r_{2}^{5} r_{1} \cdot r_{2}\end{array}$


exchange processes, eg. $F_{1}$ with scalar meson exchange, $F_{2}$ with vector meson exchange, etc. From that point of view, the 5 covariant form of $\dot{F}$ is appealing. However, since $\dot{F}$ is defined in the full Dirac space of two nucleons, only the positive energy matrix elements of $\dot{F}$ are controlled by $N$ scattering data. The amplitudes involving negative energy components are unconstrained, leaving a large ambiguity in the Dirac optical potential that is constructed from $\dot{F}$. This makes conclusions drawn from IAl highly questionable, especially conclusions concerning virtual Nii pair processes which are explicitly contained in the negative energy matrix elements of the optical potential.

As an lliustration of how this ambiguity in the optical potential can manifest itself, the choice of using pseudoscalar $\mathrm{rN}$ coupling or pseudovector $\mathrm{rN}$ coupling to represent pion exchange is noted. Even though pseudoscalar coupling results in different negative energy matrix elements in $\dot{U}$ than for pseudovector coupling, the positlve energy matrix elements are the same in each case. Thus each choice is perfectly legitimate since data only constrain positive energy matrix elements. However, it is well known that virtual pair contrioutions arising from the use of pseudoscalar coupling in NN scattering need to be suppressed. This suggests that pseudovector coupling should be used in any meson exchange model describing p-Nucleus scattering. Indeed, IAl describes p-Nucleus elastic scattering observables quite well except at $300 \mathrm{MeV}$ where the need to suppress virtual $N \bar{N}$ pairs is greatest. At this energy, pseudoscalar coupling produces overly large scalar and vector 
potentials. But when pseudovector coupling is used in IAl, by replacing the pseudoscalar covariant with a pseudovector one, an improvement is found in the resulting predictions of p-Nucleus scattering observables. It vould therefore appear that pseudovector $\pi N$ cowpling needs to be incorporated into whatever representation is used for the Lorentz invariant amplitudes, ambiguous as they are.

In view of the flaws of IAl, a generalized Impulse approximation known as IA2 has been developed recently $y^{31}$. Since the ambiguity inherent in IAl can only be removed by constructing a dynamical model in the full Dirac space of two nucleons, IA2 incorporates such a model to deal with this problem. The negative energy matrix elemeris of the Dirac optical potential are based upon the one-boson exchange model of van Fasssen and $T_{j} \mathrm{n}^{33}$. Channel coupling between $N$, $N \Delta$, and $\Delta \Delta$ states are included with $\pi, \varepsilon, \eta, \rho, \delta$ and $\omega$ meson exchange in order to describe inelasticity in the $\mathrm{NN}$ channel. Also, IA2 automatically includes pseudovector $\pi \mathrm{N}$ coupling. This dynamical model gives complete sets of NN amplitudes which predict a conplete set of negative energy matrix elements in addition to the positive energy matrix elements. In this way, the ambiguity in the choice of representations of Lorentz invariant amplitudes is removed. At the present time, no other dynamical model exists to fix $\bar{F}$ completely.

The form of $\dot{F}$ used in IA.2 is given by ${ }^{31}$

$$
\dot{F}=\sum_{p_{1}^{\prime}} \sum_{p_{2}^{\prime}} \sum_{p_{1}} \sum_{p_{2}} \mu_{1}^{\rho_{1}^{\prime}}\left(\vec{p}_{1}^{\prime}\right) \mu_{2}^{\rho_{2}^{\prime}}\left(\vec{p}_{2}^{\prime}\right) \dot{F}_{1}^{\prime} p_{2}^{\prime} p_{1} p_{2} \mu_{1}^{\rho_{1}}\left(\vec{p}_{1}\right) \mu_{2}^{p_{2}}\left(\vec{p}_{2}\right)
$$

where $p_{i}=+$ or - for positive and negative energy initial states $i=1$ 
and 2, and similarly for the final states denoted with primes. Thus there are 16 p spin sectors to the matrix describing the scattering of two nucleons. For each o spin sec:or there are eight independent amplitudes in $\dot{F} P_{1}^{\prime} P_{2}^{\prime} P_{1} P_{2}$. This glves 128 independent amplitudes of which only 44 are independent on mass shell. The $A^{\prime} s$ are covariant profection operators which separate the positive and negative energy sectors of the full Dirac space of two nucleons. The result of all this is a five term optical potential of the form

$$
U(r)=s(r)+r^{0} V(r)-i \vec{\alpha} \cdot \dot{r} T(r)-\left(S_{1 s}(r)+r^{0} V_{1 s}(r)\right) \vec{\sigma} \cdot \vec{L}(194)
$$

with scalar, vector and tensor potentials, $S, V$, and $T$ and scalar and vector spin-orbit potentials $S_{15}$ and $v_{15}$. The potential generated from IA1 does not contain the spin-orbit potentials. When IA2 is used to predict p-Nucleus elastic scattering observables in the low energy region where IA1 failed, IA2 is seen to be $a$ significant improvement over IAI.

The relativistic description of elastic scattering has thus seen much progress. The original approach of Dirac phenomenology using twelve fitting parameters has been updated with a parameter free relativistic impulse approximation that is quite successful. However, there are problems that need to be faced. For example, the success of the Dirac approach has been attributed to virtual $N \bar{N}$ pair processes. But in order for this effect to contribute to the scattering process, two successive scattering locations vould have to be within a distance of $1 / 2 M_{p}=.1 f m$. The virtual $N \bar{N}$ pair could not 
possibly exist nside a volume of $\left(1 / 2 M_{p}\right)^{3}$ unless nucleons could be treated as pointlike. It is thus entirely possible that the relativistic theory is successfully describing the data for the wrong reason ${ }^{34,35}$.

The current state of p-Nucleus elastic scattering can be summed up very easily: We have a non-relativistic scattering theory that does not work at energies less than $800 \mathrm{MeV}$ and a relativistic theory that appears to work quite well in the energy range 200-800 MeV for reasons that are questionable or at least not understood. Both theorists and experimentalists are faced with the challenges presented by the current situation. Theorists need to solve the puzzle associated with $N \bar{N}$ pairs. Work toward this end may require going down to the level of quarks and gluons. Investigations into virtual $q \bar{q}$ pair processes as 1llustrated in figure 52 are underway ${ }^{36}$. Experimentally, more data needs to be taken. In particular,

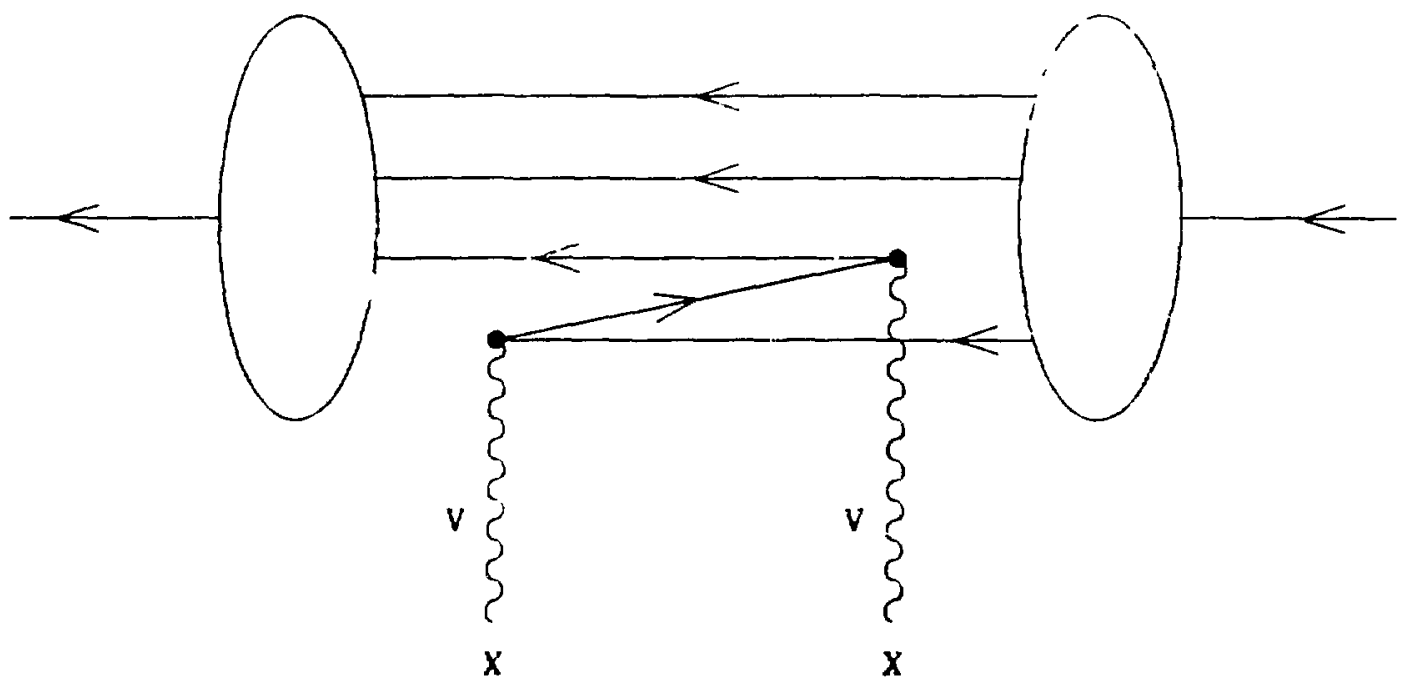

Fig. 52

Z-Graph showing virtual $q \bar{q}$ pair processes. 
measurement of the absolute phase of the nuclear amplitude in combination with the three independent observables $d \sigma / d Q, A_{y}$, and 0 would completely determine the scattering amplitude and provide information with which to test relativistic theories. The absolute phase has not been measured in the energy region where large discrepancy exists between non-relativistic and relativistic theories. Perhaps measurement of the absolute phase could have as big an impact as that which occurred when 0 was first ieasured to reinforce doubts about KMT. It is hoped that this discussion will stimulate work, both theoretically and experimentally, so that implications that would stem from knowledge of the so far unmeasured absolute phase could be brought into focus.

\section{V-C. PROBLEMS IN OBTAINING HIGH OUALITY SMALL ANGLE DATA}

As discussed in the previous section, nuclear information from small angle data can be used to study differences between various theoretical models. In order to be able to draw conclusions and make restrictions on theoretical models one must have data of sufficient quality such that the absolute uncertainty in the data is smaller than that given by differences in the theoretical models. Since uncertainties in some models give rise to $-5 \%$ uncertainty in the predicted differential cross section and existing data have uncertainties in the absolute normalization of 5-10\%, steps must be taken to bring the experimental error down. Therefore an examination of the factors contributing to the error in the absolute uncertainty 
of the measured differential cross section and how to reduce the error that each factor contributes is in order.

For a polarized proton beam on a spin zero target, the measured elastic scattering yield for normal beam is

$$
Y^{N}=I^{N} \varepsilon^{N} T \frac{d \sigma}{d \ell} \Delta Q\left(1+P A_{y}\right)
$$

Here $I^{N}$ is the number of bean particles incident on target, $\varepsilon^{N}$ is the efficiency of the system to detect elastic events, $T$ is the number of nuclei in the target, $\Delta Q$ is the solid angle subtended, $P$ is the polarization of the beam, and $A_{y}$ is the analyzing power. Similarly for reverse beam

$$
Y^{R}=I^{R} \varepsilon^{R} T \frac{d \sigma}{d \Phi} \Delta Q\left(1-P A_{y}\right)
$$

Combining eqns. (195) and (196) gives

$$
\frac{d \sigma}{d \bar{Q}}=\frac{1}{2 T \Delta Q}\left(\frac{Y^{N}}{\varepsilon^{N} I^{N}}+\frac{Y^{R}}{\varepsilon^{R} I^{R}}\right)
$$

The error in the cross section will then be the contributions of each element of eqn. (197) added in quadrature

$$
(\delta \sigma)^{2}=\sum\left(\frac{\partial \sigma}{\partial i}\right)^{2}(\delta i)^{2}
$$


where o is the elastic differential cross section, and the sum is over the 1 elements $T, \Delta Q, Y^{N}, Y^{R}, \varepsilon^{N}, \varepsilon^{R}, I^{N}, I^{R}$. As was discussed in section IV-C, the errors associated with efficiencies are very small. The errors in the yields $Y^{N}$ and $Y^{R}$ are minimized with patience in accumulating more events. The error associated $w$ th $T$ is about 1 - 5\%. Using a target thickness uniformity gauge 7 , this error can be reduced to much less than percent. This leaves the errors stemming from solid angle variations and beam particle counting which ve will discuss in more detail here.

In section IV-A the method used to obtain the scattering angle (and thus the solid angle) of the elastically scattered proton vas described. This method, namely using a polynomial form for describing the scattering angle, is not as good as some of the methods discussed below. Thus the error coning from the polynomial method $(-2 \%)$ can be reduced by using an alternative method.

One vay to determine the solid angle of scattered particles entering the hRS spectrometer is to place solid angle acining apertures at the spectroneter entrance. This eliminates the need for calibrating the horizontal and vertical scattering angles at the target using rods and slits. However, other problems arise in using this method. In order to bin the data in small angular bins $(2-3 \mathrm{mr})$, at least 10 apertures would need to be at the entrance to the spectrometer. Gaps between apertures vould unnecessarily decresse the total solid angle accepted by the spectrometer. In addition to the uncertainty introduced in putting such apertures in place, there is the problem of scattering off of the eoges of the apertures. For 
this reason, it is always best not to use physical objects to define angular acceptance.

A way around these problems would be to measure the soldd angle of the scattered particle directly. This could be done by simply placing delay-line drift chambers in the scattering chamber of the HRS just before the entrance of the spectrometer. However, problems exist here too. Chambers placed in the scatering chamber vould require dead regions in them for the beam to pass through. In addition, since the inside of the scattering chamber is in vacuum, any drift chambers put inside would have to be vacuum tight. Scattered particles passing through such drift chambers would also give rise to a loss in resolution at the focal plane. Also multiple scattering of the beam passing through the drift chambers may cause a broad beam spot at the focal plane.

Perhaps an even better method vould be to map out the solid angle on the focal plane by measuring directly the solid angle of recoil particles before performing the small angle experiment. This could be done as follous. First place the spectrometer at some large angle, say $20^{\circ}$. Using pp elastic scattering, measure the solid angle of the recoil particle with drift chambers placed just outside of the scattering chamber as shown in fig. 53. A one to one correspondence between the solid angle of the recoll particle measured dirertly and the solid angle of the scattered particle seen on the focal plane could then be obtained. This allows the boundartes of angular bins to be defined nore precisely than they might otherwise be.

The other problen that contributes more than any other factor to 


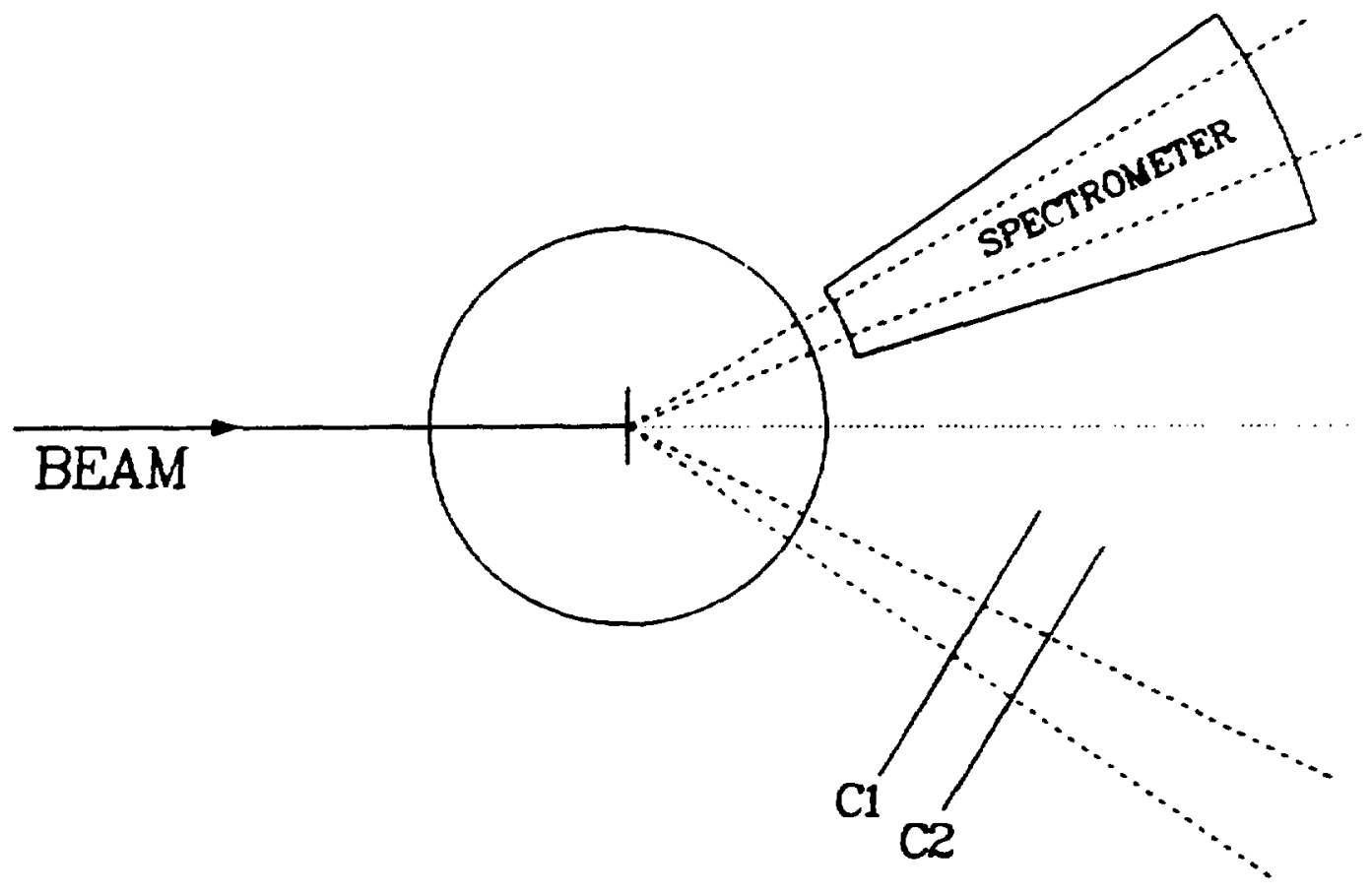

Fig. 53

Method of determining the solid angle of scattered particles.

the uncertainty in the absolute normalization of the data is that of measuring the beam intensity. There are many devices used to measure the bean intensity, eg. ion chambers, Faraday cup, etc. He will discuss here a particular method for measuring the beam intensity that vas investigated when some beam time became avallable for this purpose. This method uses two plastic scintillators placed in the scattering chamber as shown in fig. 54 to count the number of beam particles. Since several beam particles may pass through the scintillators and be counted as only one particle, a statistical nethod vas used to determine the actual number of beam particles 


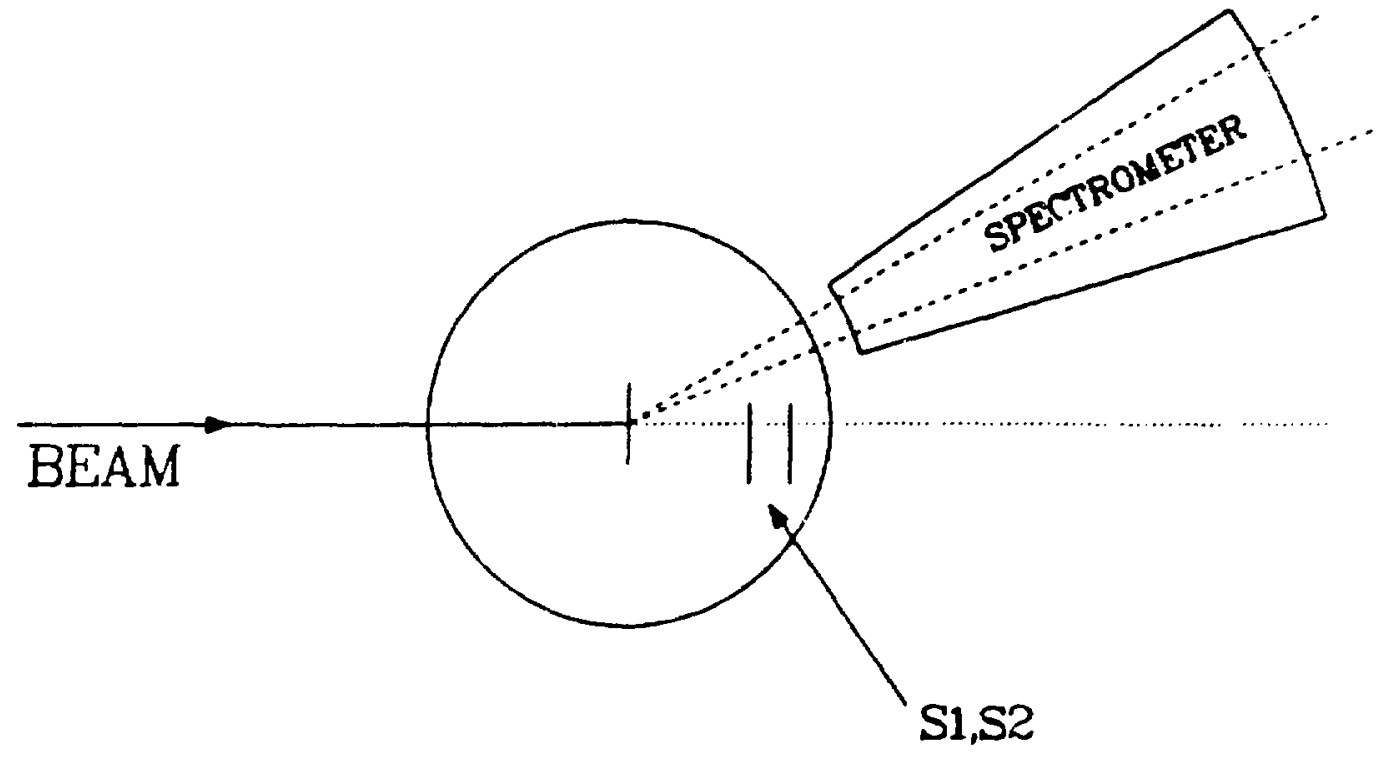

Fig. 54

Position of scintillators used to count beam particles.

passing through the system as discussed in appendix $c$. Thus we discuss here the results obtained from counting the beam in this manner.

Placing the scintillators in the scattering chamber such that the beam was completely intercepted and that the scintillators Interfered minimally with the scattered particles entering the spectrometer, was done as follows. First the two scintillators vere surveyed into place in the scattering chamber on a table which rotates about the center of the scattering chamber. As a check that the scintillators were positioned properly with respect to the 
Incident beam, the optimum position of the table vas decermined using the beam. The table vas rotated clockwise and counter-clockwise, thus moving the scintillators away from and closer to the beam respectively. By plotting the number of coincidences between $S 1$ and S2 for a given time interval as a function of the angle of the table, the optimum setting of the table angle vas determined. Fig. 55 shows a plot of coincidence counts from the scintillators per bean gate as function of the table angle. One car see quite clearly that the plot "plateaus", le. the data polnts ascend as the

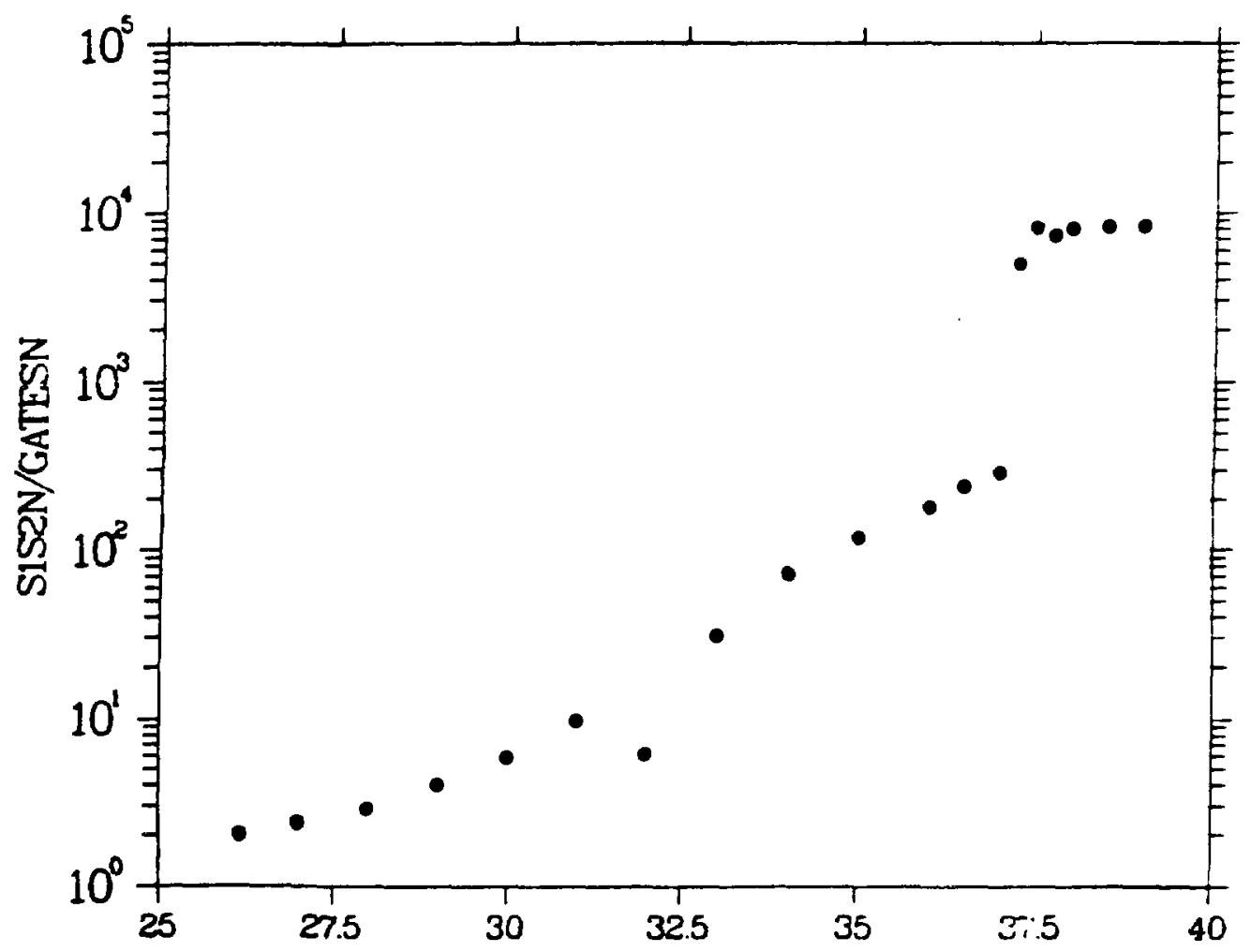

TABLE ANGLE

Pig. 55

Plot to determine the optimum setting of the table in the scattering chamber. 
scintillators move closer to the beam up to a point where a plateau is reached. It is in this plateau region that the table angle should be chosen. We chose $38^{\circ}$ for the table angle as this value assured that the beam was completely intercepted and interference with scattered particles was minimized.

Differential cross section data was taken with ${ }^{40} \mathrm{Ca}$ and ${ }^{208} \mathrm{~Pb}$ targets at $2.5^{\circ}$ and $3.25^{\circ}$ as a test of our method of counting the beam. At $2.5^{\circ}$ the scintillators that vere counting the beam in the scattering chamber did interfere somewhat with scattered particles entering the spectrometer. This is seen in the top of $\mathrm{fig}$. 56 where a dotplot of $\mathrm{X}$ vs. Y at the focal plane shows some background due to this effect smearing the spectral line. However, this background can be easily dealt with. A histogram of phi at the focal plane shows two peaks as displayed in $\mathrm{fig}$. 57. The peak on the left is due to the beam counting scintillators. By appropriately cutting off this peak, the background is essentially eliminated. This is seen in the bottom half of fig. 56 which shows $X$ vs. Y at the focal plane having cut off the left peak of fig. 57 .

Fig. 58 shows the differential cross sections with the beam having been counted in the manner fust described. These points are labeled EXP916U in the figure. Comparison of this data to the solid dot and triangle data in the figure suggests that our mettiod of counting the beam is falrly reliable. As one check, ve took data at two different beam intensities to see if our bean counting method is rate dependent. The results of this check are shown in fig. 59. In the top half of the figure the ratio of the cross sections obtained 


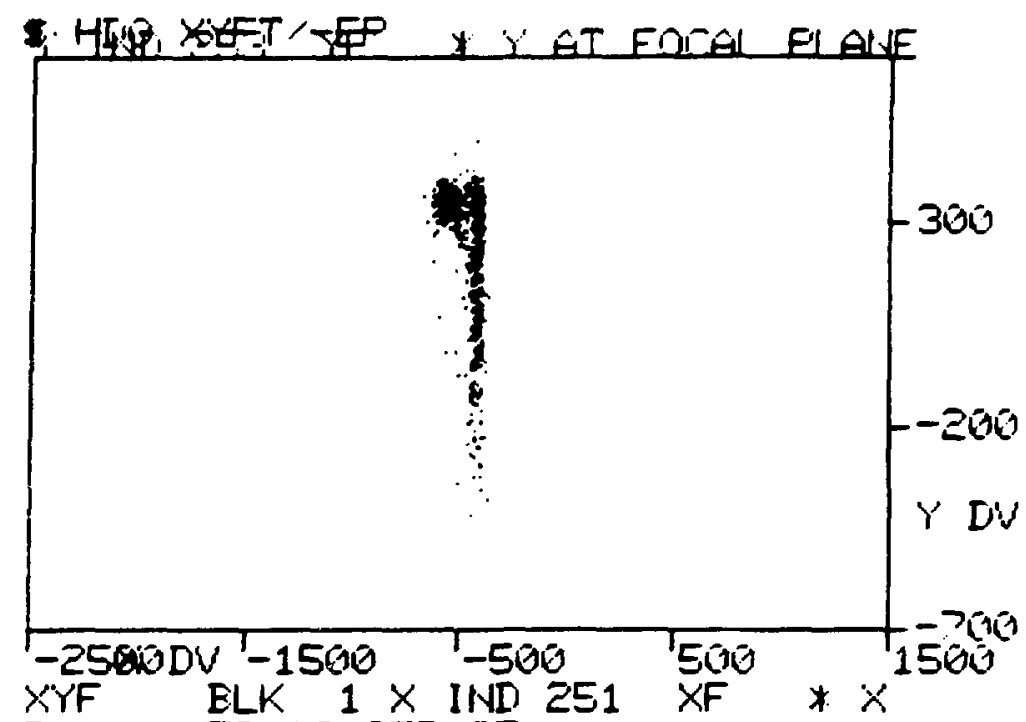

RUN $5 \overline{3} 16-S E F-87$

TST 78 12:10:54

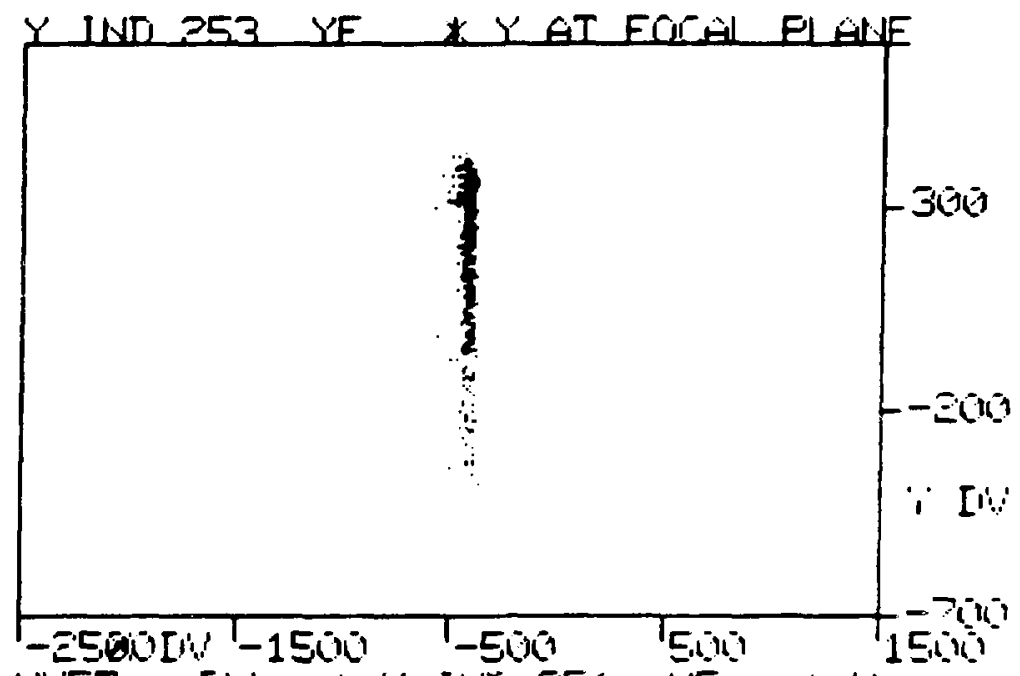

YIFT ELF I $\times$ IIIII ZEI YF * $\because$

FUII $531 E-S E F-8$ ?

TET 7S $12: 10: 58$

Fig. 56

View of focal plane with(top) and without (bottom) events that scattered from the bean counting scintillators. 


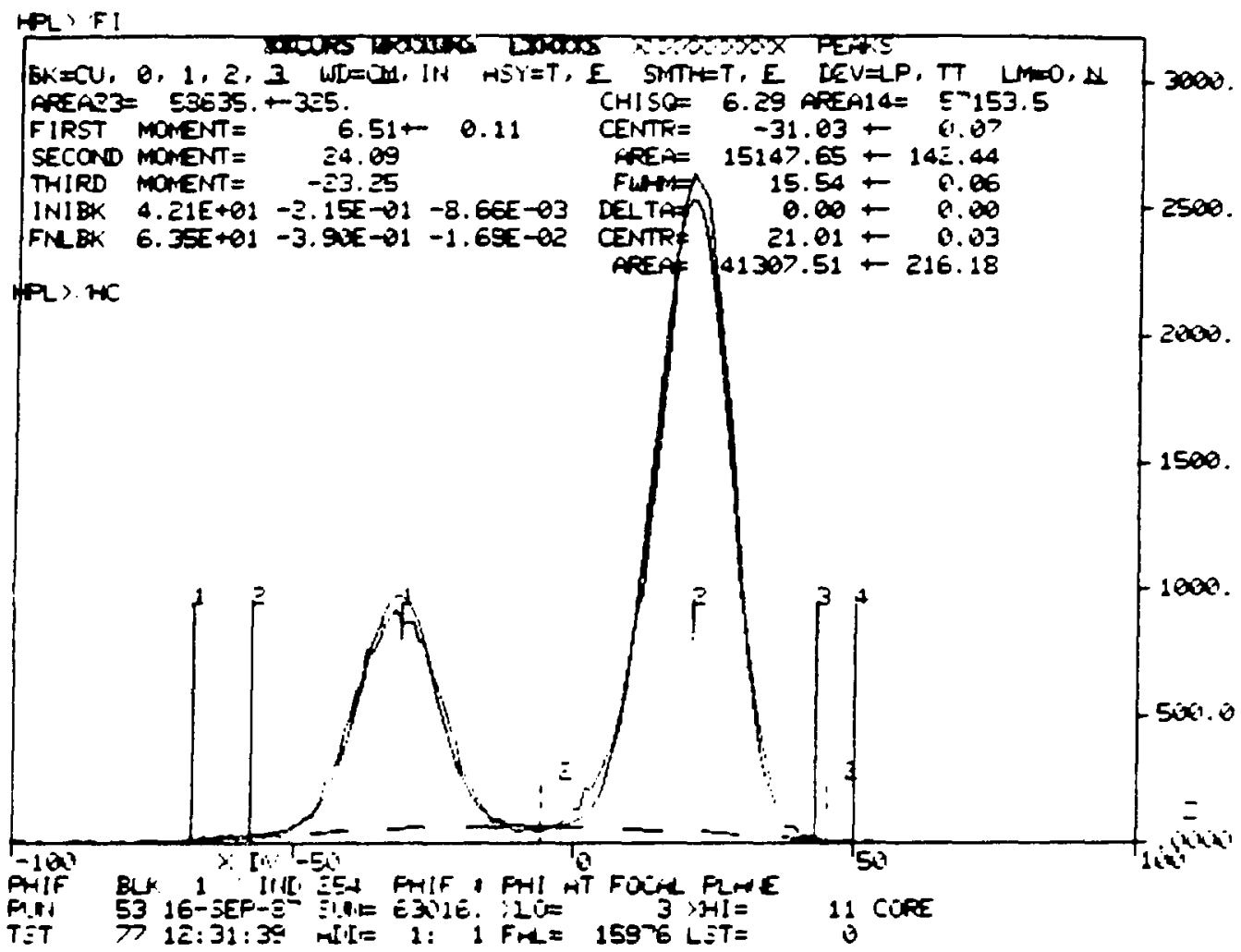

Fig. 57

Histogram of phi at the focal plane shouing two peaks. The peak at left is from the beam counting scintillators.

at an instantaneous rate of $-5.2 \times 10^{6} / \mathrm{s}$ to $-1.8 \times 10^{6} / \mathrm{s}$ is plotted vs. momentum transfer for ${ }^{40} \mathrm{Ca}$. These rates correspond to 0.49 and 0.17 average number of beam particles per nilcropulse respectively. In the botton half of the figure the ratio of daca obtained with an Instantaneous rate of $-3.6 \times 10^{6} / \mathrm{s}$ to $-2.1 \times 10^{6} / \mathrm{s}$, or 0.34 and 0.20 average number of beam particles per micropulse, is showr. for ${ }^{208} \mathrm{~Pb}$. A ratio of 1.0 would mean that the statistical counting method is rate independen? and from the figure this is nearly so. Factors that may be responsible for the value of the veighted average of the data 


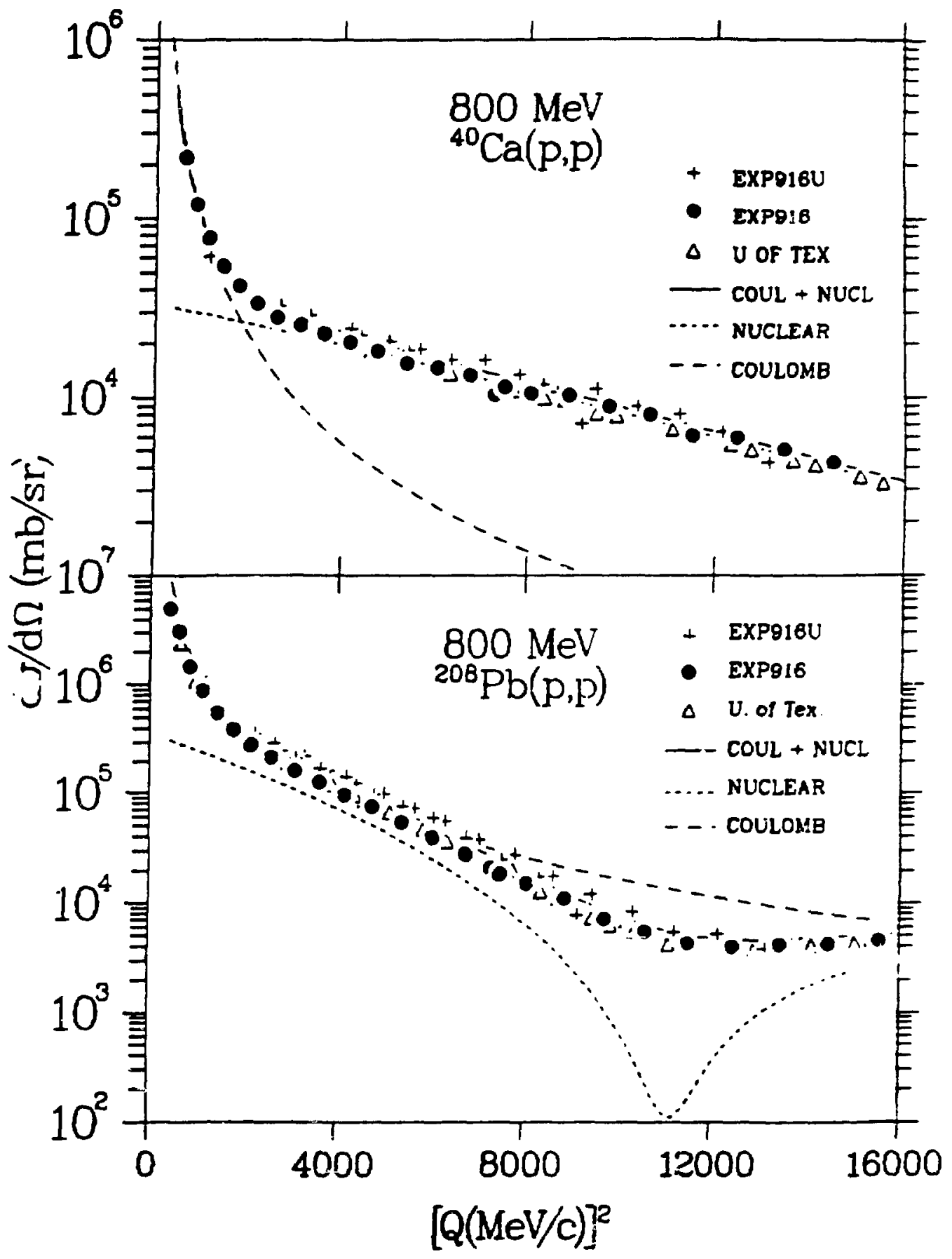

Pig. 58

Differential cross sections for protons on $10 \mathrm{Ca}$ and $201 \mathrm{~Pb}$ at $800 \mathrm{MeV}$. 


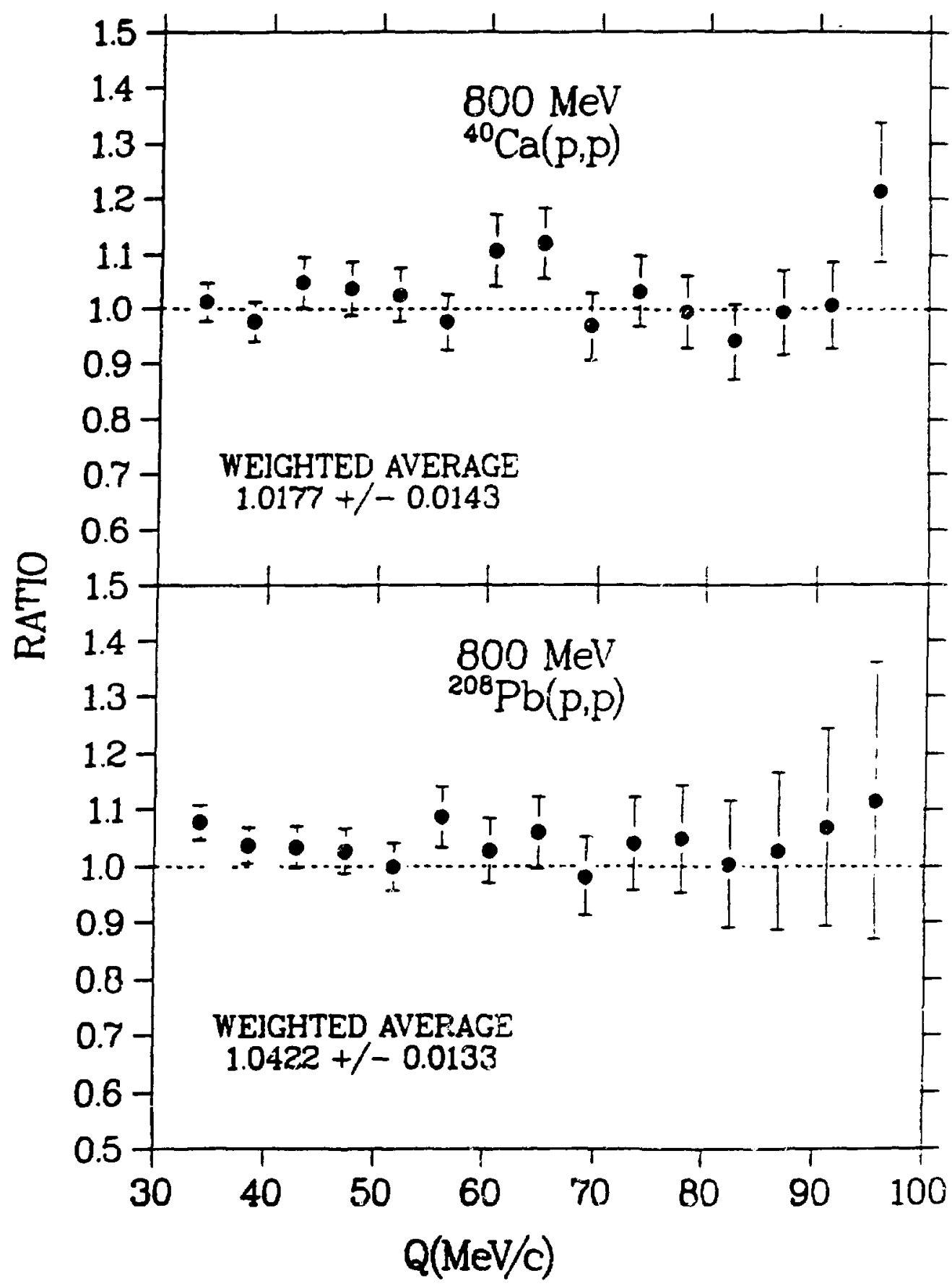

81g. 59

Ratio of differential cross sections measured at two different bean intensities. 
points being slightly greater than 1.0 include the fact that the beam used to take this data was only $95 \%$ bunched. The other $5 \%$ of the beam appeared halfway between the main beam burst. The actual beam siructure therefore consisted of $95 \%$ of the bean in micropulses $-0.25 n$ wide separated from each other by 100ns, and 57 of the beam in micropulses 50ns in time behind the nicropulses containing nost of the beam. This could be interpreted as there being two suecessive beam bursts each with a different $x, x$ being defined in appendlx $C$ as the average number of beam particles in a micropulse. Eqn.(7) of appendix $C$ vill then be

$$
N_{p}=\frac{1}{2} N_{m}\left[\left(1-\exp \left(-x_{1}\right)\right)+\left(1-\exp \left(-x_{2}\right)\right)\right]
$$

where $x_{1}$ is the average number of beam particles in the nain beam burst and $x_{2}$ is the average number of beam particles in the other $5 x$ of the beam. Going through the same procedure as done in appendix $C$, one obtains an expression similar to eqn.(12) of appendix $C$. The result is to make an overall correction of -17 to the total number of beam particles counted.

Another factor to consider in interpreting fig. 59 is the fact that when the bean intensity was changed, it was done by changing the width of a set of jaws through which the beam passed. This means that the area of the tergets iritercepting the bean changed in going from one intensity to the other. Target thickness uniformity therefore plays ar Important role in a test of this sort. Since the 
targets used were uniform to $-5 x$, a discrepancy of this order from the value of 1.0 for the ratio of $\mathrm{f} 1 \mathrm{~g}$. 59 could be attributed to this effect.

Another way of dealing with the problem of absolute normalization is to measure the differential cross section in the region where Coulomb scattering dominates. Since the Coulomb cross section is vell known, data in this region can be normalized to the relativistic Rutherford form for the differential cross iction. Thus, if one measures down to where the Coulomb contribution to the total elastic differential cross section is two orders of magnitude higher than the nuclear contribution, a normalization of $1 \%$ is achieved.

Using Coulomb scattering to get a good normalization of the data has an added benefit. The angular offset of the spectrometer is also determined from this method. This can be seen as follows. Assume for the monent that the Coulomb cross section deareases linearly with $q^{2}$ (1t doesn't really, but for the sake of argument let's assume that 1t does.) This is shown as a dashed line in the top half of fig. 60 . Let's say that we have some data represented by crosses in fig. 60 . Then if one is to normalize the crosses to the dashed line, one needs to know precisely how much to nove the data points up and precisely how much to move them to the right. For the hypothetical case we are considering, it is impossible to know this. As the arrows in the figure show, there are any different coabinations for aoving the data points up and to the right. We could adjust all the data points 


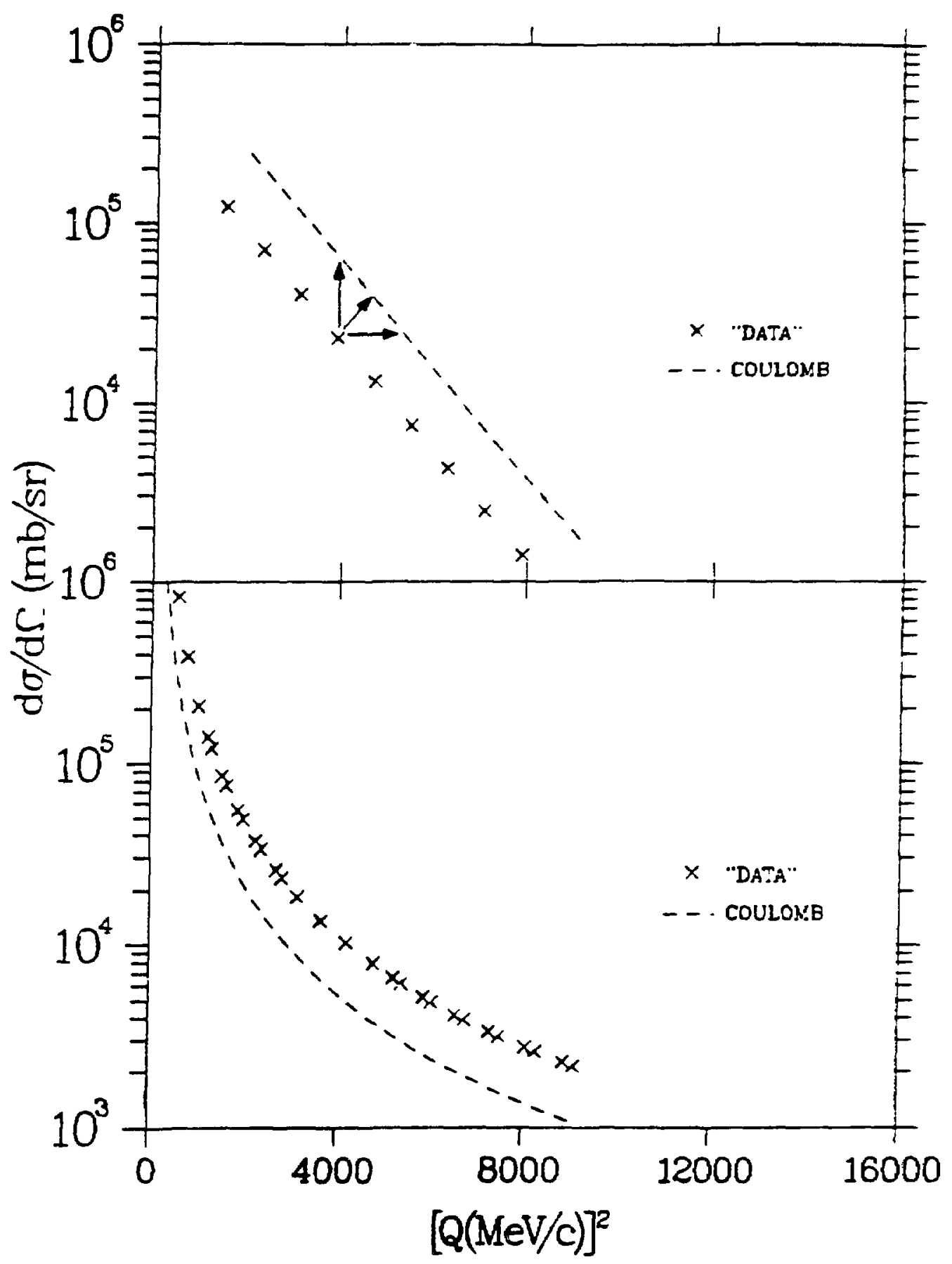

P18. 60

Illustration shoving that a unique normalization constant and angular offset can be obtained from Coulonb scattering. 
with normalization constant alone, an angular offset alone, or combinations of the two.

Fortunately, the coulomb cross section decreases as $1 / q^{4}$ as shown in the bottom of $\mathrm{fig}$. 60. Thus the ambigulity in choosing the correct combination of normalization and angular offset vanishes. There is one and only one combination that will make all of the crosses match the dashed line. This is a result of the fact that the second derlvative of the differential cross section with respect to $\mathrm{q}^{2}$ is not zero. In fact, one can show that for any function $y(x)$ used for normallzing a set of data $\left\{x_{i}, y_{i}\right\}$, the cholce of moving each of the data points by an amount $\Delta x, \Delta y$ is unambiguous as long as there is at least one point in the function $y(x)$ where $d^{2} y / d x^{2}$ is not zero. Thus, using the Coulomb cross section for normalizing data provides both an absolute normalization constant and an angular offset. 


\section{REFERENCES}

1. Igo, G., et al., LAMPF research proposal No. 916, 7/1/85

2. Perl, M., High Energy Badron Physics, (U1ley, 1974)

3. Rodberg, L., and R. Thaler, Introduction to the Quantum Theory of Scattering, (Academic, 1967)

4. Messiah, A., Quantum Mechanics, (Miley, 1958)

5. Matheus, J., and R. Valker, Mathematical Methods of Physlcs, (Benjamin/Cummings, 1970)

6. Goldberger, M., and K. Vatson, Colliston Theory, (Viley, 1964)

7. Whittaker, E., and G. Watson, Modern Analysis, (Cambridge, 1944) p. 253

8. Bethe, H., Ann. Phys. 3 (1958) 190

9. Fregeau, J., and R. Hofstadter, Phys. Rev. 99 (1955) 1503

10. Islam, M., Phys. Rev. 162 (1967) 1426

11. Irom, F., LAMPF Report LA-8648-T

12. Aebischer, D., et al., Nucl. Phys. A276 (1977) 445

13. Ericson, T., and M. Locher, Nucl. Phys. A148 (1970) 1

14. Livingston, M., LAMPF Report LA-6878-MS

15. Zeidman, B., LAMPF Report LA-4773-MS

16. Rosen, L., LAMPF Report LA-UR-85-3437

17. Hoffmann, G., et al., Phys. Rev. C21 (1980) 1488

18. Hoffmann, G., et al., Phys. Rev. C24 (1981) 541

19. Bevington, P., Data Reduction and Error Analysis for the Physical Sciences, (HcGraw-Hill, 1969)

20. Ray, L., et al., Phys. Rev. C23 (1981) 828

21. Hoffmann, G., et al., LAMPF Research proposal No. 1079, 7/6/87

22. Beyer, H.(ed.), CRC Handbook of Tables for Probability and Statistics, (The Chemical Rubber Co., 1966) 
23. Hoffmann, G., in J. Shepard(ed.) et al., "Proceedings of the LAMPF workshop on Dirac appioaches to nuclear physics", Los Alamos Conference LA-10438-C

24. Kerman, A., et al., Ann. of Phys. 8 (1959) 551

25. Hoffmann, G., et al., Phys. Rev. Lett. 47 (1981) 1436

26. Hutcheon, D., et al., Phys. Rev. Lett. 47 (1981) 315

27. Rahbar, A., et al., Phys. Rev. Lett. 47 (1981) 1811

28. Arnold, L., et al., Phys. Rev. C23 (1981) 1949

29. Tandy, P., in M. Johnson and A. Picklesimer(eds.), Relativistic Dynamics and Quark Nuclear Physics, (Wiley, 1986)

30. Vallace, S., in J. Shepard(ed.) et al., "Proceedings of the LAMPF workshop on Dirac approaches to nuclear physics", Los Alamos Conference LA-1U438-C

31. Tjon, J., and S. Wallace, U. of Md. preprint no. 87-132(1987)

32. Vallace, S., "Relativistic equation for nucleon-nucleus scattering", U. of Md. preprint

33. vanfaassen E., and J. Tjon, Phys. Rev. C30 (1984) 285

34. Cooper, E., and B. Jennings, Nucl. Phys. A458 (1986) 717

35. Thies, M., Phys. Lett. 162B (1985) 255

36. Bleszynski, E., et al., Phys. Rev. Lett. 59 (1987) 423

37. Liljestrand, R., et al., Nucl. Instr. Meth. 138 (1976) 471 
Adams, D., and M. Bleszynski, "On the relevance of the Dirac equation to the scattering of medium-energy nucleons from nuclei", Phys. Lett. 136B (1984) 10

Aebischer, D., et al., "Small-angle p-p elastic scattering at energies between 285 and $572 \mathrm{MeV}$ ", Phys. Rev. D13 (1976) 2478

Amado, R., "Analytic insights into intermediate-energy hadron-nucleus scattering", Adv. Nucl. Phys. 16 (1986) 1

Amaldi, U., et al., "Measurements of the proton-proton total cross section by means of Coulomb scattering at the CERN intersecting storage rings", Phys, Lett. 43B (1973) 231

Amaldi, U., et al., "The real part of the forward proton proton scattering amplitude measured at the CERN intersecting storage rings", Phys. Lett. 66B (1977) 390

Amos, N., et al., "Comparison of small-angle $p \bar{p}$ and Pp elastic scattering at the CERN intersecting storage rings", Phys. Lett. 128B (1983) 343

Anastasio, M., et al., "Relativistic nuclear structure physics", Phys. Rep. 100 (1983) 327

Apokin, V., et al., "Small-angle $\pi^{-}$p elastic scattering measurements", Phys. Lett. 56B (1975) 391

Apokin, V., et al., "Elastic $\pi^{+} p, K^{+} p$, and $p p$ scattering in the region of Coulomb-nuclear interference at momenta 42.5 and $52.2 \mathrm{GeV} / \mathrm{C}^{\prime \prime}$, Yad. Fiz. 25 (1977) 94

Arnold, L., et al., "Energy dependence of the $\mathrm{p}-{ }^{\circ} \mathrm{Ca}$ optical potential: a Dirac equation perspective", Phys. Rev. C25 (1982) 936

Azhgirey, L., et al., "Small-angle elastic scattering of protons on carbon nuclei at $660 \mathrm{MeV}$ ",

Nucl. Phys. 43 (1963) 213

Baier, $V .$, et al., "Experiments on Coulomb-nuclear interference in $p \bar{p}$ scattering at superhigh energy colliding beams", Nucl. Inst. Meth. 205 (1983) 107

Baillon, P., et al., "Coulomb-nuclear interference in $\pi^{ \pm} p$ and $k^{ \pm} p$ elastic scatte: ing below $3 \mathrm{GeV}$ : 
measurements, real parts and $k^{ \pm} p$ dispersion relations", Nucl. Phys. B105 (1976) 365

Barlett, M., et al., "Forward-angle elastic and quasielastic proton-nucleon cross sections and analyzing powers at $0.8 \mathrm{GeV} "$, Pliys. Rev. C27 (1983) 682

Barrette, J., and N. Alamanos, "Forward glory and the determination of the nuclear forward amplitude from heavy-ion elastic scattering", Nucl. Phys. A441 (1985) 733

Bartenev, V., et al., "Investigation of elastic proton-proton scattering in the region of the interference of the Coulomb and the nuclear interactions in the energy interval 8-70 GeV", Sov. J. Nucl. Phys. 16 (1973) 50

Bartenev, V., et al., "Real part of the proton-proton forward-scattering amplitude from 50 to $400 \mathrm{GeV}$ ", Phys. Rev. Lett. 31 (1973) 1367

Batty, C., et al., "Forward pion-nucleus elastic scattering amplitudes", Nucl. Phys. B67 (1973) 492

Bawin, M., and J. Cugnon, "Coulomb effects in nuclear collisions", Phys. Rev. C25 (1982) 387

Beznogjkh, G., et al., "Small angle proton-proton elastic scattering from 9 to $70 \mathrm{GeV} / \mathrm{c}^{\prime \prime}$, Phys. lett. 39B (1972) 411

Beznogith, G., et al., "Elastic small-angle He-p scattering in the energy interval 1.75-4.13 GeV/nucleon", Sov. J. Nucl. Pliys. 27 (1978) 380

Bialkowski, G., and S. Pokorski, "Dispersion relation calculation for the real part of the forward $p p$ and pp scattering amplitude in the high-energy region", Nuovo Cimento A57 (1968) 219

Binon, F., et al., "Coulomb-nuclear interference in $\pi^{-}-12 \mathrm{C}$ elastic scattering", Nucl. Phys. B33 (1971) 42

Blanpied, G., et al., "Elastjc scattering of $0.8-\mathrm{GeV}$ protons from ${ }^{12} \mathrm{C}, 58 \mathrm{Ni}$, and $208 \mathrm{~Pb}^{\prime \prime}$,

Phys. Rev. Lett. 39 (1977) 1447

Bleszynski, E., et al., "Optical potential approach in scattering of hadrons from light nuclei",

Phys. Rev. C33 (1986) 1228

Block. M., et al., "Forward hadronic pp and $\bar{p} p$ elastic scattering amplitudes: analysis of existing data and extrapolations to colljder energies", 
Phys. Lett. 120B (1983) 224

Block, M., et al., "Neas forward $p-p$ and $\bar{p}-p$ elastic scattering: slope analysis of existing data", Phys. Let t. 120B (1983) 229

Boudrie, R., et al., "On-line polynomial optimization for high resolution spectrometers",

IEEE Trans. Nucl. Sci., NS-26, (1979) 4588

Bourrely, C., et al., "Analytic parameterization of high-energy forward scattering amplitude (I). pp and pp scattering", Nucl. Phys. B61 (1973) 513

Bronzan, J., et al., "Obtaining real parts of scattering amplitudes directly from cross section data using derivative analyticlty relations", Phys. Lett. 49B (1974) 272

Brown, G., et al., "Relativistic effects in nuclear physics", Comm. Nucl. Part. Phys. 17 (1987) 39

Burkhardt, H., Dispersion Relation Dynamics, (North-Hollaño, 1969)

Carter, A.s and D. Bugg, "The real part of the proton-neutron forward scattering amplitude", Phys. Lett. 20 (1966) 203

Clark, B., et al., "Optical-model partial-wave analysis of 1-GeV proton-nucleus elastic scattering", Phys. Rev. C7 (1973) 466

Clark, B., et al., "Dirac-equation impulse approximation for intermediate-energy nucleon. nucleus scattering", Phys. Rev. Let t. 50 (1983) 1644

Clark, B., et al., "Dirac phennmenology and the nuclear optical model", AIP Conf. Proc. 97 (1983) 260

Cromer, A., "Direct-interaction model calculation of high-energy proton-carbon scattering", Phys. Rev. 113 (1959) 1607

Czyz, W., L. Lesniak, and H. Wolek, "The role of Coulomb interaction in elastic and almost elastic scattering of hadrons from nuclei", Nucl. Phys. 819 (1970) 125

Czyz, W., "High-energy scattering from nuclei", Adv. Nucl. Phys. 4 (1971) 61

Das, K., and B. Deo, "Coulomb-nuclear interference and extraction of nuclear parameters",

Phys. Rev. C26 (1982) 211 
Das, K., and B. Deo, "Nuclear parameters for $\pi \pm 12 \mathrm{C}$ scattering from Collomb-nuclear interference", Phys. Rev. C26 (1982) 2678

Dobrovolsky, A., et al., "Small angle pp scattering at energies from 650 to $1000 \mathrm{HeV",} \mathrm{Nucl.} \mathrm{Phys.} \mathrm{B214} \mathrm{(1983)} 1$

Dumbrais, 0., "Model-independent determination of the real parts of the spin-independent amplitudes in forward PP scattering", Sov. J. Nucl. Phys. 13 (1971) 626

Dumbrais, 0., "A theoretical model-independent determination of the real parts and zeros of the pp, $\bar{p} p$ forward spin-1ndependent scattering amplitudes", Nucl. Phys. B46 (1972) 164

Eden, R., Aigh Energy Collisions of Elementary Particles, (Cambridge University Press, London, 1967)

Fajardo, L., et al., "Real part of the forvard elastic nuclear amplitude for $p p, \bar{p} p, \pi^{+} p, \pi^{-} p, K^{+} p$, and $K^{-} p$ scattering between 70 and $200 \mathrm{GeV} / \mathrm{c}^{\prime \prime}$, Phys. Rev. D24 (1981) 46

Foley, K., et al., "Small-angle elastic pion-proton scattering at high energies and the real part of the scattering amplitude", Phys. Rev. Lett. 14 (1965) 862

Foley, K., et al., "Experimental test of the pion-nucleon forvard dispersion relations at high energies",

Phys. Rev. Lett. 19 (1967) 193

Foley, K., et al., "High-energy, small-angle, p-p and $\bar{p}-p$ scattering, and $p-p$ total cross sections",

Phys. Rev. Lett. 19 (1967) 857

Foley, K., et al., "Experimental test of the pion-nucleon forward dispersion relations at high energies", Phys. Rev. 131 (1969) 1775

Frohlich, J., et al., "Coulomb effects in pion-calcium scattering", Phys. Lett. 1218 (1983) 235

Frohlich, J., et al., "Effects of a refined Coulomb treatment of $\pi \pm$ - deuteron elastic scattering observables",

Nucl. Phys. 1435 (1985) 738

Giacomelli, G., "Pion-nucleon elastjc scattering" La Revista del Nuovo Cimento 2 (1970) 297 (Serie Prima)

Gross, D., et al., "Real part of the $p-p, p-d$, and $p-n$ 
forvard scattering amplitudes form 50 to $400 \mathrm{GeV}$ ", Phys. Rev. Let t. 41 (1978) 217

Heckrotte, V., "Analysis of the small-angle elostic scattering of high-energy protons by carbon", Phys. Rev. 101 (1956) 1406

Highland, V., "Some practical remarks on multiple scattering", Nucl. Inst. Keth. 129 (1975) 497

Highland, V., "Erratum", Nucl. Inst. Meth. 161 (1979) 171

Bllgevoord, J., Dispersion Relations and Causal Description, (North-Hollar,d, 1960)

Holdeman, J., and R. Thaler, "Remarks on charged-particle scattering", Phys. Rev. 139 (1965) b1186

Horowitz, C., "Relativistic Love-Franey model: covariant representation of the $n$ interaction for n-nucleus scat tering", Phys. Rev. C31 (1985) 1340

Hynes, M., et al., "Relativistic (Dirac equation) effects in microscopic elastic scattering calculations", Phys. Rev. C31 (1985) 1438

Igo, G., et al., "Elastic different1al cross sections and analyzing powers for $\vec{p}+10,12,14,11 \mathrm{Ca}$

at $0.8 \mathrm{GeV}$, Phys. Let t. B1B (1979) 151

Irom, F., et al., "Elastic p-p scattering at $796 \mathrm{MeV}$ in the Coulomb-nuclear interference region", Phys Rev. C25 (1982) 373

Jaminon, M., "The Schrodinger equivalent complex potential and the Dirac phenomenology", AIP Conf. Proc. 97 (1983) 324

Jenni, P. et al., "p̄p and pp forward elastic scattering between 4 and $10 \mathrm{GeV} / \mathrm{c}^{n}$, Nucl. Phys. B129 (1977) 232

Kanazawa, A., and $M$. Sugawara, "I terative determination of analytic continuation and its application to the pp forward amplituden, Phys. Lett. 40B (1972) 265

Khuri, N., and T. Kinoshita, "Forward scattering amplitude and univalent functions", Phys. Rev. 140 (1965) 706

Khuri, N., and T. Kinoshita, "Real part of the scattering amplitude and the behavior of the total cross section at high energies", Phys. Rev. 137 (1965) 720 
K1rlllova, L., et al., "The real part of the pp elastic scattering amplitude at $2,4,6,8$ and 10 BeV", Sov. Phys. JETP 23 (1966) 52

Klein, L., Dispersion Relations and the Abstract Approach to Field Theory, (Gordon and Breach, 1961)

Kok, L., et al., "Formulas for the 8 -shell-plus-Coulomb potential for all partial vaves", Phys. Rev. C26 (1982) 2381

Lechanoine, C., et al., "Polarization phenomena in nucleon-nucleon forward scattering", Nuovo Cimento 56A (1980) 201

Lilley, J., et al., "Excitation of 170 and 180 scattered by $20^{\circ} \mathrm{Pb}$ in the Coulomb-nuclear interference region", Phys. Let t. 128B (1983) 153

Linssen, L., et al., "Measurement of antiproton-proton small-angle elastic scattering at low momentum", Nucl. Phys. A469 (1987) 726

Linssen, L., et al., "Measurement of antiproton-proton small-angle elastic scattering at low momentum", CERN-EP/87-36

Locher, M., "Relativistic treatment of structure in the Coulomb interference problem", Nucl. Phys. B2 (1967) 525

Lumpe, J., and L. Ray, "Correlation effects in the relativistic impulse approximation treatment of proton-nucleus elastic scattering", Phys. Rev. C35 (1987) 1040

Lumpe, J., and L. Ray, "Virtual nñ pair processes as a relativistic correction to second order nultiple scattering theory", Univ. of Tex. preprint

Lynch, W., et al., "Relativity, nuclear polarizability, and screening in sub-Coulomb elastic scattering", Phys. Rev. Lett. 48 (1982) 979

McNe1l, J., et al., "Impulse-approximation Dirac optical potential", Phys. Rev. Lett. 50 (1983) 1439

McNeil, J., et al., "Impulse approximation NN amplitudes for proton-nucleus interactions". Phys. Rev. C27 (1983) 2123

Melissinos, A., and S. Olsen, "Physics (and technique) of gas jet experiments", Phys. Rep. C117 (1975) 77 
Mur, V., et al., "Coulomb problem with short-range forces (exact solution)", Yad. Fiz. 42 (1985) 1470

Murdock, D., and C. Horowitz, "Microscopic relativistic description of proton-nucleus scattering", Phys. Rev. C35 (1987) 1442

Mutchler, G., et al., "Nuclear-Coulomb interference in $\pi^{+}$- I2C scattering", Phys. Rev. C9 (1974) 1198

Mutchler, G., et al., "Nuclear-Coulomb interference in $\pi^{ \pm}$- i60 scattering", Phys. Rev. C11 (1975) 1873

Nakamura, K., et al., "Absorption and forward scattering of antiprotons by $C, A l$, and $C u$ nuclei in the region 470-880 MeV/c", Phys. Rev. Lett. 52 (1984) 731

Nakano, K., "Two-potential formalisms and the Coulomb-nucleat interference", Phys. Rev. C26 (1982) 1123

Newton, R., Scattering Theory of Vaves and Particles,

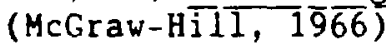

Nigam, B., "Information about the proton-proton scattering matrix from Coulomb interference",

Prog. Theor. Phys. 24 (1960) 407

Nikitin, V., et al., "Possible methods of studying interference between Coulomb and nuclear scattering in the elastic collision of particles of energy $10^{10} \mathrm{eV}^{\prime \prime}$, Sov. Phys. JETF 19 (1964) 1088

Pauletta, G., et al., "Differential cross sections and analyzing powers for pp elastic scattering at $1.46 \mathrm{GeV} / \mathrm{c}$ in the Coulomb-nuclear interference region", Phys. Rev. C27 (1983) 282

Picklesimer, A., "Antisymmetrized elastic scattering and optical potential theory", Phys. Rev. C24 (1981) 1400

Ray, L., et al., "Analysis of $0.8-\mathrm{GeV}$ polarized-proton elastic scattering from $208 \mathrm{~Pb}, 90 \mathrm{Zr}, 50 \mathrm{Ni}$, ancl $12 \mathrm{C} "$, Phys. Rev. C18 (1978) 1756

Ray, L., "Neutron isotopic density differences deduced from $0.8 \mathrm{GeV}$ polarized proton elastic scattering", Phys. Rev. C19 (1979) 1855

Ray, L., et al., "Coulomb interaction in multiple scattering theory", Phys. Rev. C22 (1980) 1454

Ray, L., and G. Hoffmann, "800 MeV protons as probes of 
neutron transition densities", Phys. Rev. C27 (1983) 2133

Ray, L., and G. Hoffmann, "Proton isotonic density differences from $0.8 \mathrm{GeV}$ proton-nucleus elastic scattering", Phys. Rev. C27 (1983) 2143

Ray, L., "First order interpretation of optical potentials", AIP Conf. Proc. 97 (1983) 121

Ray, L., and G. Hoffmann, "Relativistic and nonrelativistic impulse approximation descriptions of $300-1000 \mathrm{MeV}$ proton + nucleus eilastic scattering", Phys. Rev. C31 (1985) 538

Ray, L., et al., "Sensitivity of $P_{p o l}+A_{p o l}$ elastic spin observables to relativistic aspects of nuclear structure", Phys. Rev. Let t. 56 (1986) 2465

Rix, J., and R. Thaler, "Separation of strong and electromagnetic effects in charged-particle scattering", Fhys. Rev. 152 (1966) 1357

Rost, E., and J. Shepard, "Nucleon-nucleus inelastic scattering using a relativistic impulse approximation with exchange", Phys. Rev. C 35 (1987) 681

Sakurai, J., "Speculatinns on the real part of the forward pp $(p \bar{p})$ scattering amplitude",

Phys. Rev. Lett. 16 (1966) 1181

Schwaller, P., et al., "Proton total cross sections on ${ }^{1} \mathrm{H}$, ${ }^{2} \mathrm{H},{ }^{4} \mathrm{He},{ }^{9} \mathrm{Be}, \mathrm{C}$ and $\mathrm{O}$ in the energy range 180 to $560 \mathrm{MeV}$ ", Nucl. Phys. A316 (1979) 317

Schwandt, P., "Proton and nelltron elastic scattering between 80 and $1000 \mathrm{MeV} "$, AIP Conf. Proc. 97 (1983) 89

Scott, M., et al., "Nuclear-Coulomb interference in $\pi^{+}-{ }^{12}$ C scattering", Phys. Rev. Lett. 28 (1972) 1209

Scott, W., "The theory of small-angle multiple scattering of fast charged particles", Rev. Mod. Phys. 35 (1963) 232

Screaton, G., Dispersion Relations, (Oliver and Boyd Ltd., 1961)

Shafranova, M., "Elastic small-angle pp, pd, and pn scattering at high energies", Sov. J. Part. Nucl. Vol.5, No.3 (1975) 259

Shepard, J., et al., "Relativistic impulse approximation for p-nucleus elastic scattering", Phys. Rev. Lett. 50 (1983) 1443

Shepard, J., "Dirac phenomenology for nuclear reactions", 
AIP Conf. Proc. 97 (1983) 288

Shirkov, D., et al., Dispersion Theories of Strong Interactions at Low Energy, (Wiley, 1969)

Sidhu, D., and U. Sukhatme, "Real parts of forward elastic hadronic amplitudes", Phys. Rev. D11 (1975) 1351

Soding, P., "Real part of the proton-proton and proton-antiproton forward scattering amplitude at high energies", Phys. Lett. $\underline{8}$ (1964) 287

Solov'ev, L., "Small angle scattering of charged particles", Sov. Phys. JETP 22 (1966) 205

Sukhatme, U., et al., "Extensions of the derfvative dispersion relations for amplitude analyses", Phys. Rev. D12 (1975) 3431

Thies, M., "On the relation between relativistic and non-relativistic mean-field theories", Phys. Let t. 166B (1986) 23

Thies, M., "Dirac vs. Schroedinger approach in proton-nucleus scattering: a simple model", AIP Conf. Proc. 156 (1986) 692

Tjon, J., and S. Wallace, "Meson theory of the Dirac impulse approximation", Phys. Rev. Let t. $\underline{54}$ (1985) 1357

Tjon, J., and S. Wallace, "Meson theoretical basis for Dirac impulse approximation", Phys. Rev. C32 (1985) 267

Tjon, J., and S. Wallace, "Symmetric, Lorentz invariant NN amplitude: Yukawa representation",

Phys. Rev. C35 (1987) 280

Velichko, G., et al., "Elastic $p{ }^{4}$ He scattering at small angles at $992 \mathrm{MeV}$ ", Sov. J. Nucl. Phys. 35 (1982) 154

Velichko, G., et al., "Elastic scattering of protons on helium nuclei in the energy range 700-1000 $\mathrm{MeV} "$, Yad. Fiz. 42 (1985) 1325

Vernov, Y., "The asymptotic form of the elastic scattering amplitude and the ratio of its real and imaginary parts", Sov. Phys. JETP 26 (1968) 130

Vorobyov, A., et al., "Filastic pp-scattering in the Coulomb interference region in the momentum range 1.1 to $1.7 \mathrm{GeV} / \mathrm{c}$ ", Phys. Lett. 41B (1972) 639

Walker, G., et al.(eds.), "Proceedings of the international 
conference on ant1-nucleon and nucleon-nucleus interactions", Telluride, Co. March 18-21 1985

Hest, G., and D. Yennie, "Coulomb interference in highenergy scattering", Phys. Rev. 172 (1968) 1413

Urlekat, A., et al., "Elastic pp scattering at $1.463 \mathrm{GeV} / \mathrm{C}$ In the Coulomb interference region", Phys. Lett. 97B (1980) 33

Zayachki, V., et al., "The real part of the amplitude for elastic pd-scattering at an energy of $70 \mathrm{GeV}$ ", Sou. J. Null. Phys. 15 (1972) 528

194 


\section{Appendix A:}

The BRS solid angle in the small angle region

The solid angle acceptance of the HRS at small scattering angles deserves some discussion as the problem of calculating it at large angles is trivial in comparison. At large scattering angles, the solid angle for a given angular bin is nearly constant from one bin to the next, while for small scattering angles it changes dramatically. To see this, we must consider the geometry of the situation. First let's consider the case of the spectrometer sitting at zero degrees. Figure A shows the scattering of an incident beam particle. The trajectory of the scattered particle is the vector $\mathbb{A}$
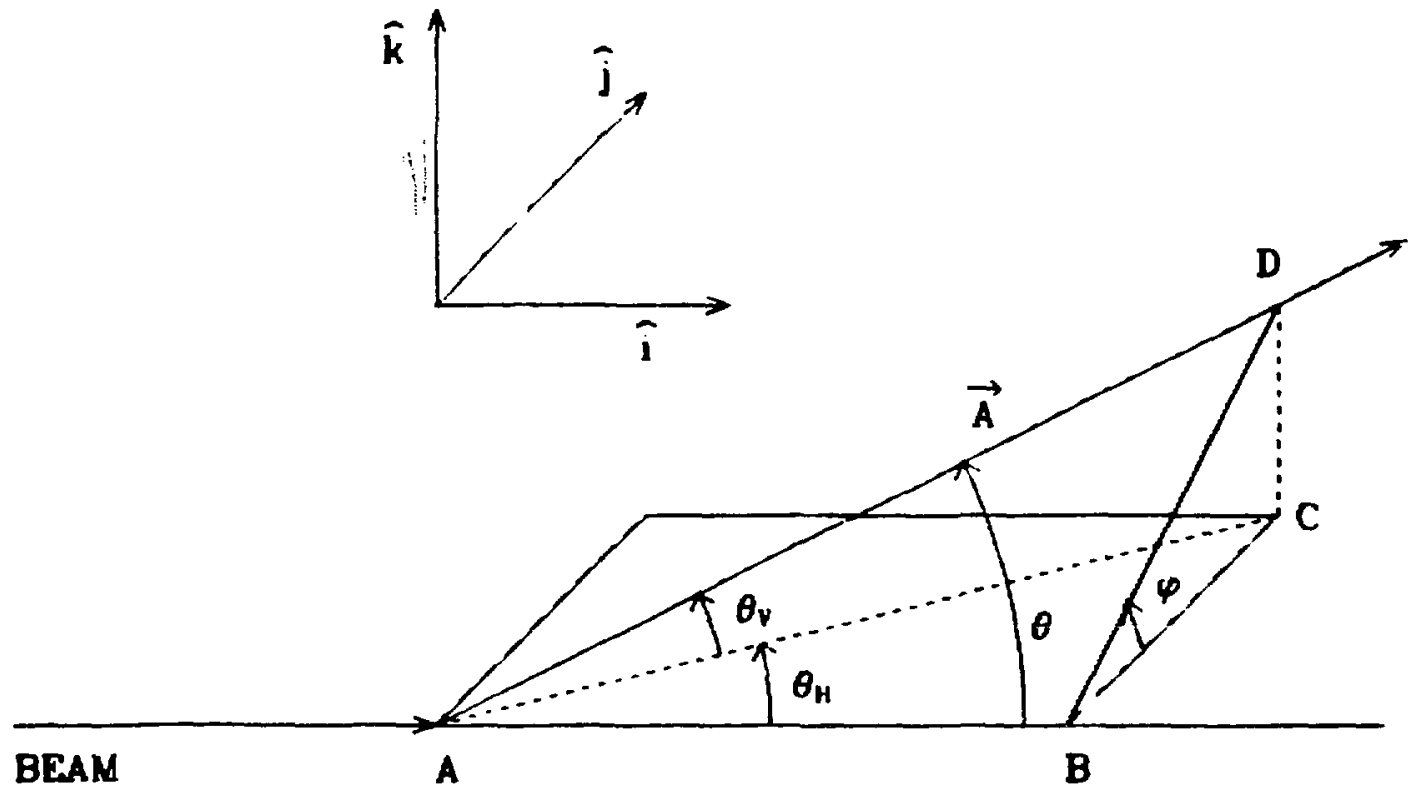

Fig. A

Scattering of a particle in the lab frame. $\theta_{\mathrm{H}}$ is the angle described by $\underline{B A C}, Q_{V}$ by $\underline{C A D}$, by $\underline{C B D}$, and $\theta$ by $\underline{B A D}$. 
going from point $A$ to point $D$. The polar angle $\theta$ is described by BAD and the azimuthal angle by $C B D$. These two angles are calculated from the two angles that are measured at the BRS: $\theta_{H}$, the horizontal scattering angle described by $\underline{B A C}$ and $\theta_{V}$, the vertical scattering angle described by $\mathrm{CAD}$. Therefore, $\theta$ and must be written in terms of $\theta_{\mathrm{H}}$ and $Q_{\mathrm{V}}$. To do this, we vrite the vector describing the scattered particle trajectory as the unt vector $\vec{A}$

$$
\star-\cos \theta_{\mathrm{V}} \cos \theta_{\mathrm{H}} \dot{\mathrm{i}}+\cos \theta_{\mathrm{V}} \sin \theta_{\mathrm{H}} \dot{j}+\sin \theta_{\mathrm{V}} \dot{\mathrm{k}}
$$

Then the polar angle $\theta$ vill be given by

$$
\tan \theta=\frac{\left|A_{j k}\right|}{\left|A_{1}\right|}
$$

where $\left|A_{j k}\right|$ is the magnitude of $A$ in the $j-k$ plane

$$
\begin{gathered}
A_{j k}=\cos \theta_{v} \sin \theta_{\mathrm{H}} j+\sin \theta_{v} \dot{k} \\
\left|A_{j k}\right|=\left(\cos ^{2} \theta_{\mathrm{V}} \sin ^{2} \theta_{\mathrm{H}}+\sin ^{2} \theta_{\mathrm{V}}\right)^{1 / 2}
\end{gathered}
$$

and $\left|A_{i}\right|$ is the magnitude of $\vec{A}$ in the 1 direction

$$
\begin{aligned}
& A_{i}=\cos \theta_{V} \cos \theta_{\mathrm{H}} \mathrm{i} \\
& \left|A_{\mathrm{i}}\right|=\cos \theta_{\mathrm{V}} \cos \theta_{\mathrm{H}}
\end{aligned}
$$

Thus eqn. (2) is 


$$
\tan \theta=\left(\tan ^{2} \theta_{\mathrm{H}}+\frac{\tan ^{2} \theta_{\mathrm{V}}}{\cos ^{2} \theta_{\mathrm{H}}}\right)^{1 / 2}
$$

The azimuthal angle will be

$$
\sin \phi=\frac{\left|A_{k}\right|}{\left|A_{j k}\right|}=\frac{\sin \theta_{V}}{\left(\cos ^{2} \theta_{V} \sin ^{2} \theta_{H}+\sin ^{2} \theta_{V}\right)^{1 / 2}}
$$

Since $\sin \theta$ is seen to be

$$
\sin \theta=\frac{\left|A_{j k}\right|}{|A|}
$$

sin $\phi$ can also be written as

$$
\sin \phi=\frac{\left|A_{k}\right|}{|A| \sin \theta}=\frac{\sin \theta_{v}}{\sin \theta}
$$

The case of the spectrometer sitting at some angle $\theta_{S}$ can be obtained from the zero degree case of figure $A$ by simply rotating the vector $\vec{A}$ about the $\vec{k}$ axis by the angle $\theta_{S}$ to get a new trajectory described by the vector $\vec{A}$. 


$$
\vec{X}^{\prime}=\left(\begin{array}{ccc}
\cos \theta_{S} & -\sin \theta_{S} & 0 \\
\sin \theta_{S} & \cos \theta_{S} & 0 \\
0 & 0 & 1
\end{array}\right)\left(\begin{array}{l}
A_{J} \\
A_{j} \\
A_{k}
\end{array}\right)
$$

$$
-\cos \theta_{V} \cos \left(\theta_{H}+\theta_{S}\right) \dot{i}+\cos \theta_{v} \sin \left(\theta_{H}+\theta_{S}\right) j+\sin \theta_{v} \dot{k}
$$

Comparing eqn. (3) is ih eqn. (1) ve see that the equations describing the zero degree case are still valid if one merely makes the transformation $\theta_{\mathrm{H}} \rightarrow \theta_{\mathrm{H}}+\theta_{\mathrm{S}}$ everywhere.

The solld angle in the small angle region behaves very differently than at large angles due to the vider range of values that the azimuthal angle can have at saall angles. This comes about because of the vertical angular scceptance of the HRS. The range of values that $\theta_{v}$ can have $15-2^{\circ}<\theta_{v}<2^{\circ}$ as governed by the vertical angular acceptance of the spectrometer. If $\theta_{v}=2^{\circ}$, then froa eqn. (8) we must have $\theta \geq 2^{\circ}$. Considering a range $c^{r} \rightarrow$, which can also be called the out-of-plane scattering angle, $\left(-\phi_{\max },+\phi_{\max }\right)$ $\phi_{\max } \leq 90^{\circ}$, then from eqn.(B) ve obtain the following expressions for $\phi_{\max }:$

$$
\begin{aligned}
\phi_{\max } & =\sin ^{-1}\left(\frac{\sin 2^{\circ}}{\sin \theta}\right) & & \theta \geq 2^{\circ} \\
& =90^{\circ} & & \theta<2^{\circ}
\end{aligned}
$$

where it is assumed in the first expression that $\theta_{v}-2^{0}$ is allowed for the scattering angle $\theta$. Figure $B$ shous the maxinum out-of-plane 


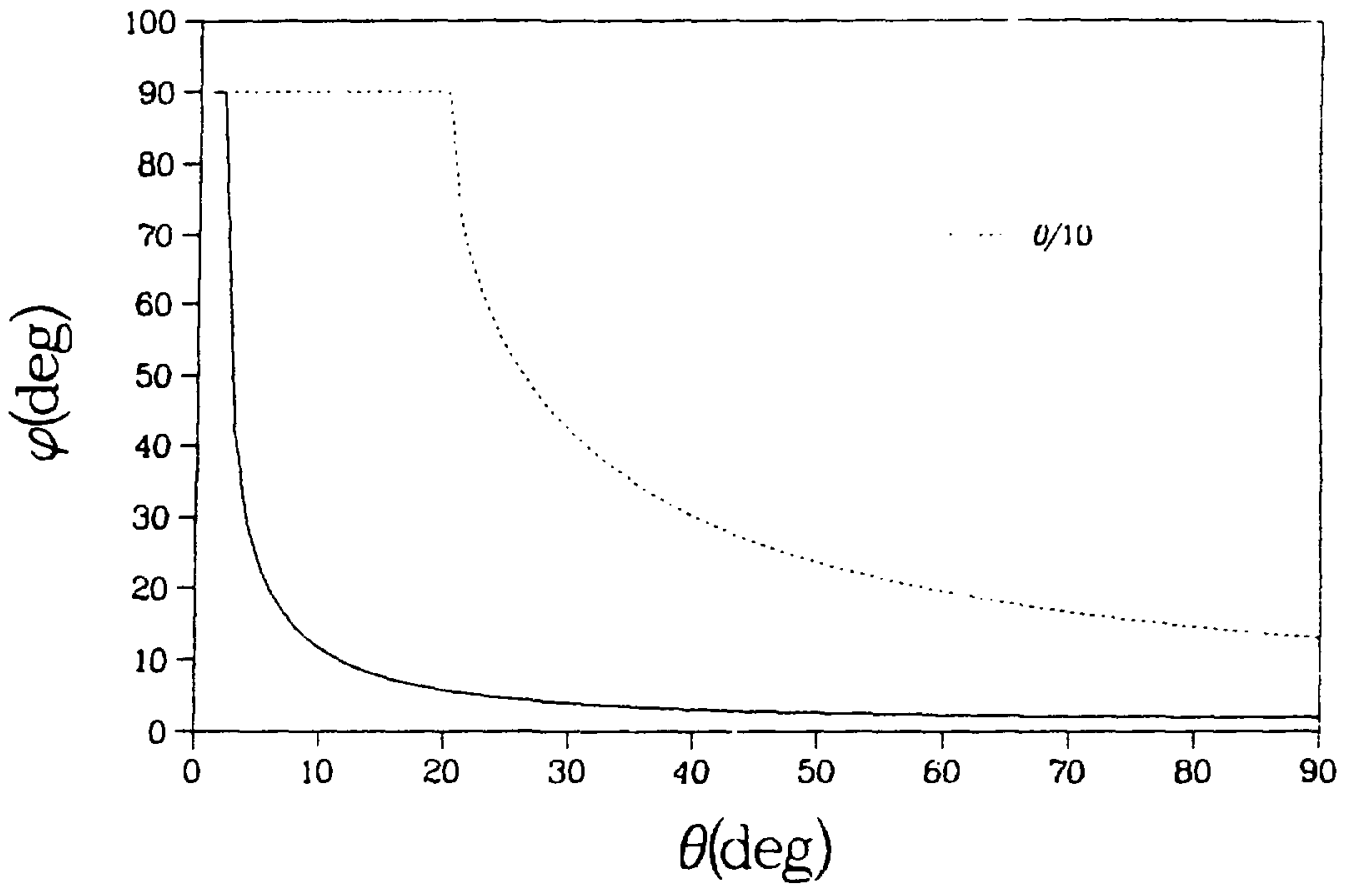

Fig. B

Maximum out-of-plane scattering angle versus the polar angle $\theta$.

The dashed line is an expanded view of $\theta$ from $0^{\circ}$ to $9^{\circ}$.

scattering angle as a function of the polar scattering angle. Clearly, for small polar angles, the out-of-plane scattering angle is quite large, whish not only causes the solid angle subtended to change rapidly, but also leads to large corrections to spin observables measured in this region.

In addition to the range of $Q_{V}$ being restricted by $\pm 2^{\circ}$, the horizontal angle $\theta_{H}$ is also restricted by the horizontal angular acceptance of the spectrometer. The range of values that $\theta_{\mathrm{H}}$ can have is $-1.2^{\circ}<\theta_{\mathrm{H}}<1.2^{\circ}$. Since the values of both $\theta_{\mathrm{H}}$ and $\theta_{\mathrm{V}}$ are 
restriesed to lie in their respective ranges, it is instructive to look at the solid angle at several different spectrometer angle settings. Figures $C, D, E$, and $F$ illustrate the solid angle acceptance at $\theta_{S}=0^{\circ}, 1.2^{\circ}, 2^{\circ}$, and $4^{\circ}$ respectively. In each figure, the solid angle acceptance of the spectrometer is the rectangle defined by BCGFB. The arcs contained in the rectangle are arcs of constant $\theta$. AD defines the upper limit of $\theta_{V}=2.0^{\circ}$ and EH defines the lower lint of $Q_{V}=-2.0^{\circ}$. Similarly $\underline{B F}$ is the lower limit of $\theta_{\mathrm{H}}=-1.2^{0}$ and $\underline{\mathrm{CG}}$ is the upper $11 \mathrm{mit}$ of $\theta_{\mathrm{H}}=1.2^{\circ}$. The vertical dashed line corresponds to $\theta_{\mathrm{H}}=0^{\circ}$ and the horizontal dashed line to $Q_{y}=0^{\circ}$.

As the solid angle contained in the acceptance of the

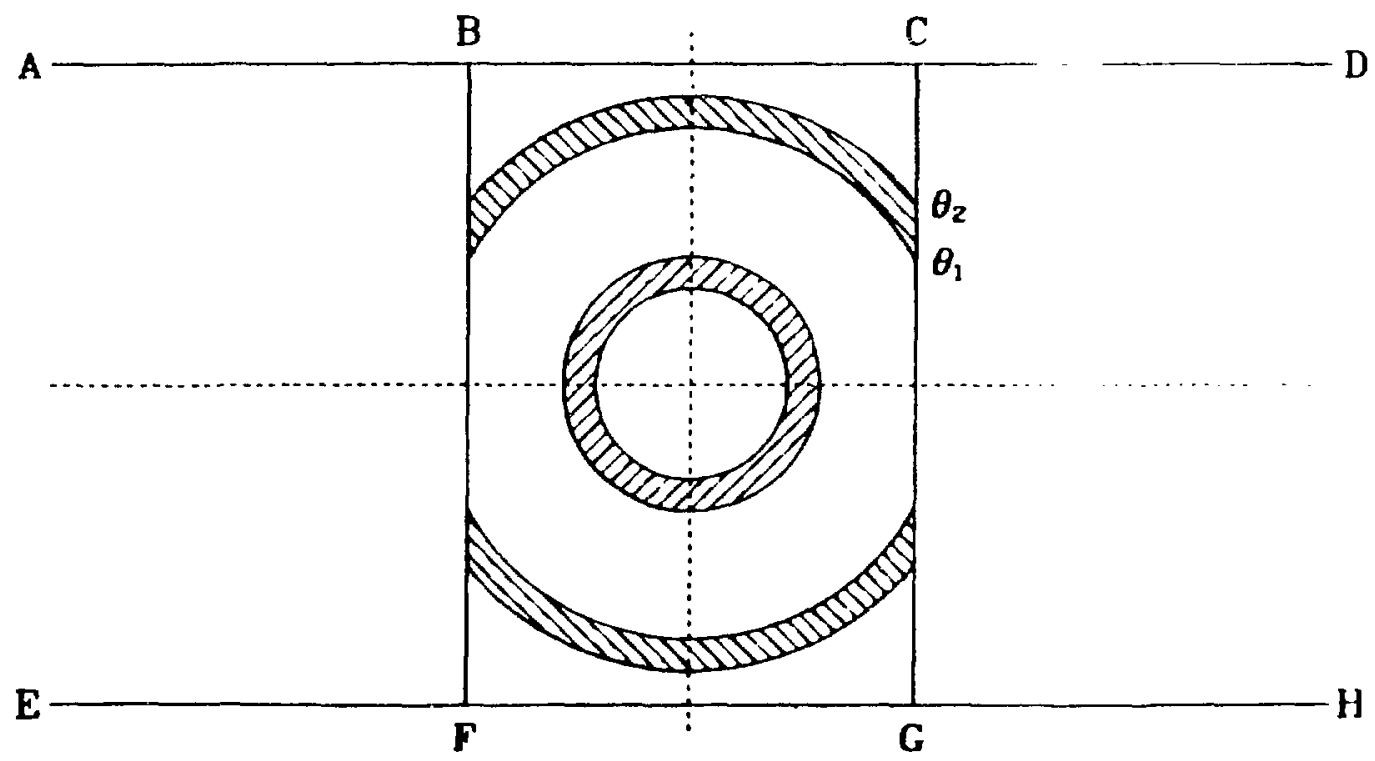

Fig. C

The solid angle acceptance of the HRS at $\theta_{S}=0^{\circ}$. 


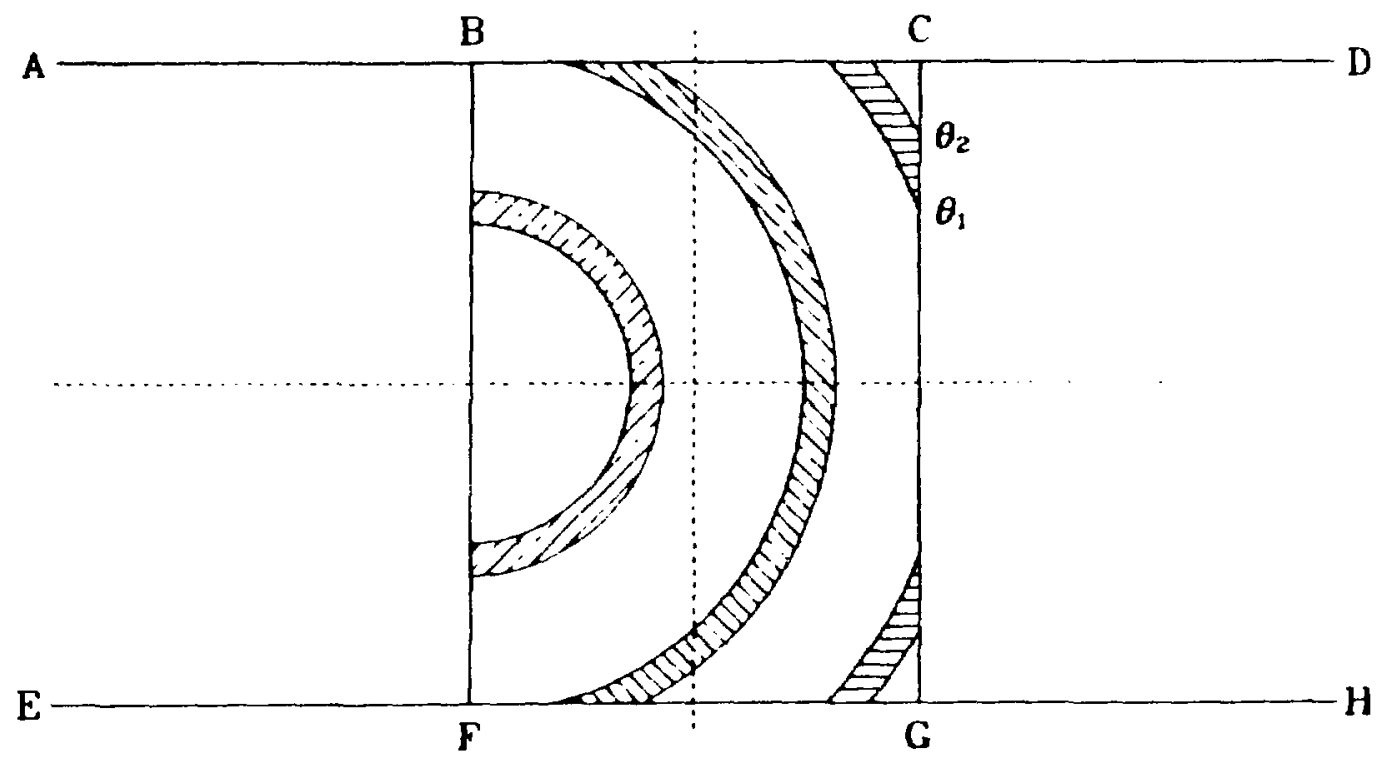

Fig. D

The solid angle acceptance of the ARS at $\theta_{S}-1.2^{\circ}$.

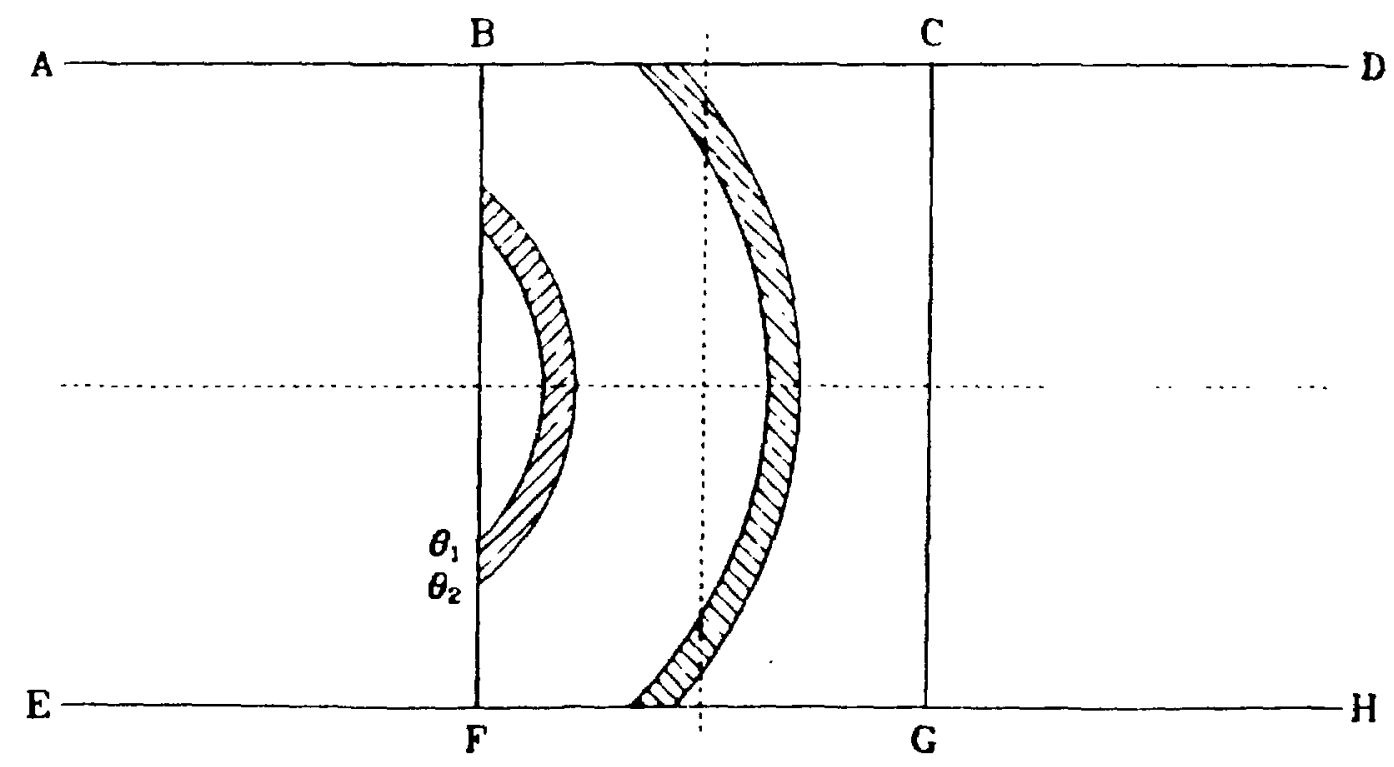

Fig. E

The solid angle acceptance of the HRS at $\theta_{S}=2^{0}$. 


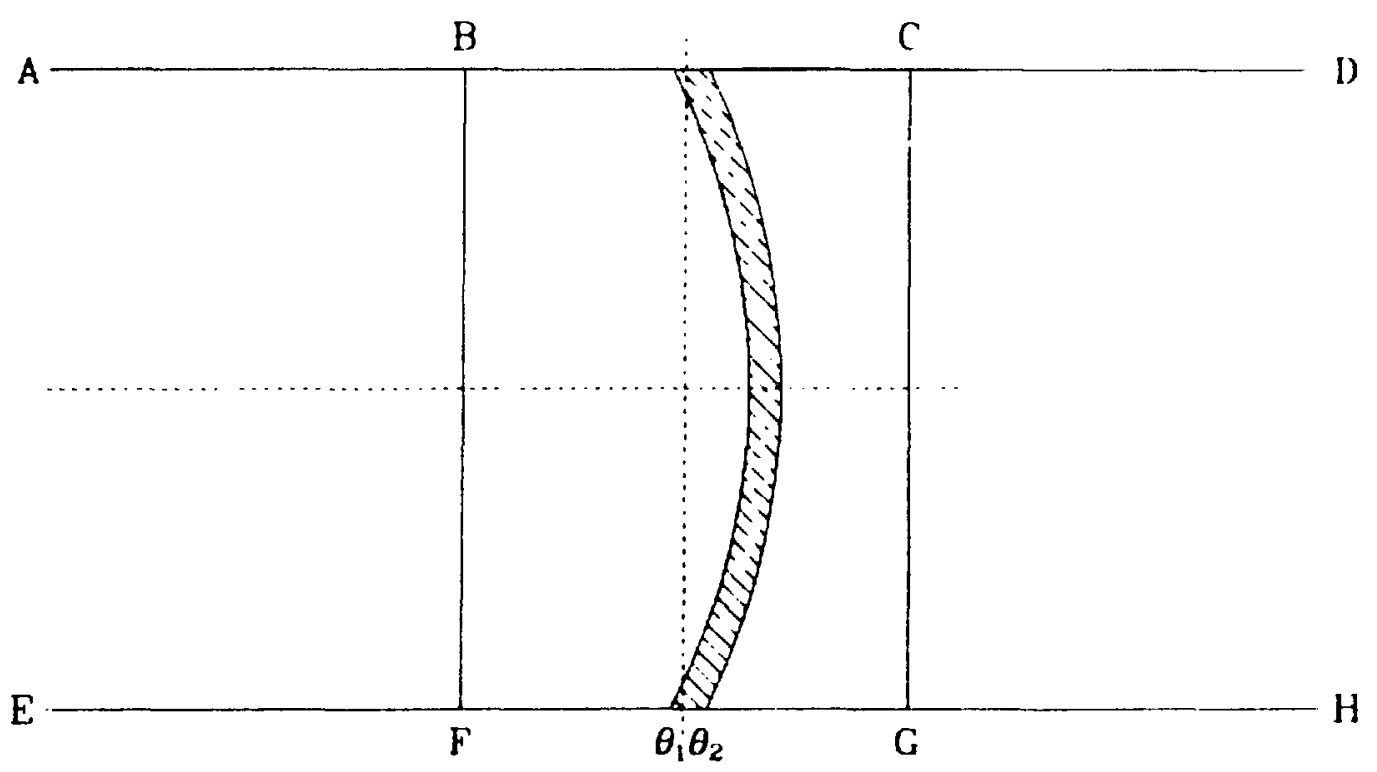

Fig. F

The solid angle acceptance of the HRS at $\theta_{S}=4^{\circ}$.

spectrometer is symmetric about the horizontal dashed line in the figures, the solid angle will be defined as

$$
\Omega=2 \int_{\theta_{1}}^{\theta_{2}} d \theta \sin \theta \int_{\phi_{1}}^{\phi_{2}} d \phi
$$

where both $\phi_{1}$ and $\phi_{2}$ are less than or equal to $\pi$. It is clear from the figures that $\phi_{1}$ and $\phi_{2}$ will be functions of $\theta$ and $\theta_{S}$. The $\phi$ range for the $\theta_{V}, \theta_{H}$ ranges $\left(-2.0^{\circ}, 2.0^{\circ}\right)$ and $\left(-1.2^{0}, 1.2^{0}\right)$ respectively can be investigated analytically as a function of $\theta_{S}$. Note that we can derive a formula for $\phi$ in terms of $\theta$ and $\theta_{\hat{H}}$

$$
\cos \phi=\frac{\tan \theta_{\mathrm{H}}^{\prime}}{\tan \bar{\theta}}
$$


where $\theta_{H}=\theta_{H}+\theta_{S}$. This indicates limits for $\$$ from the condition that $\theta_{H}$ must have a value between $\theta_{S}-1.2^{\circ}$ and $\theta_{S}+1.2^{\circ}$ and the fact that $\left|\theta_{\mathrm{H}}^{\prime}\right| \leq \theta$. For $\theta>\left(\theta_{\mathrm{H}}^{\prime}\right)_{\max }=\theta_{\mathrm{S}}+1.2^{0}$ we have

$$
\phi_{1}=\cos ^{-1}\left(\frac{\tan \left(\theta_{\mathrm{S}}+1.2^{\circ}\right)}{\tan \theta}\right)
$$

For a given $\theta$, we also have a maximum $\phi$ given by

$$
\phi_{2}=\cos ^{-1}\left(\frac{\tan \left(\theta_{S^{-1.2}}\right)}{\tan \theta}\right)
$$

and therefore
a) $\theta_{\mathrm{S}}>1.2^{0}$
$\phi_{2}<\pi / 2$
b) $\theta_{S}=1.2^{0}$
$\phi_{2}=\pi / 2$
c) $\theta_{\mathrm{S}}<1.2^{\circ}$
$\pi \geq \phi_{2}>\pi / 2$

$w_{1} \phi_{2}=\pi$ for $\theta<1.2^{0}-\theta_{S}$ for case $c$. As discussed earlier, the range of $\theta_{y}$ from $-2.0^{\circ}$ to $2.0^{\circ}$ also sets 1 imits on $\phi$, since the value of $\phi$ must also satisfy eqn.(8). This means that for $\theta>2.0^{\circ}$ we must have either

$$
\phi_{2}<\sin { }^{-1}\left(\frac{\sin 2^{\circ}}{\sin \theta}\right)
$$

or 


$$
\phi_{2}>n-\sin { }^{-1}\left(\frac{\sin 2^{\circ}}{\sin \theta}\right)
$$

In tables $A, B, C$, and $D$ we list analytic expressions for the $\phi$ ranges for the cases of figures $C, D, E$, and $F$. $\theta_{\max }$ in the tables is given by

$$
\tan ^{2} \theta_{\max }=\tan ^{2}\left(\theta_{S}+1.2^{\circ}\right)+\frac{\tan ^{2}\left(2^{\circ}\right)}{\cos ^{2}\left(\theta_{S}+1.2^{\circ}\right)}
$$

and $\theta^{\prime}$ is given by

$$
\tan ^{2} \theta^{\prime}=\tan ^{2}\left(\theta_{S}-1.2^{\circ}\right)+\frac{\tan ^{2}\left(2^{\circ}\right)}{\cos ^{2}\left(\theta_{S^{-1.2}}\right)}
$$

where $\theta^{\prime}$ is the minimum scattering angle for which $\theta_{v}$ can equal $2^{\circ}$. The solid angle is thus obtained by using the appropriate limits of $\phi_{1}$ and $\phi_{2}$ as discussed above in eqn. (11). The integration for the cases of $\phi_{1}=0$ and $\phi_{2}=\pi$ are trivial. For the other cases there are two types of integrals to be worked out. Type 1 is of the form

$$
I_{1}=\int_{\theta_{1}}^{\theta_{2}} d \theta \sin \theta \cos ^{-1}\left(\frac{A}{\tan \theta}\right)
$$

where $A=\tan \left(1.2^{\circ}+\theta_{S}\right)$ or $\tan \left(1.2^{\circ}-\theta_{S}\right)$ as the case may be. This integral can be shown to be 
Table A - Range of $\phi$ for $\theta_{S}=0^{\circ}$.

Range of $\theta$

$$
\phi_{2}-\phi_{1}
$$

$0 \leq \theta \leq 1.2^{\circ}$

$\pi$

$1.2^{\circ}<\theta \leq 2.0^{\circ}$ $\cos ^{-1}\left(\frac{\tan \left(-1.2^{\circ}\right)}{\tan \theta}\right)-\cos ^{-1}\left(\frac{\tan \left(1.2^{\circ}\right)}{\tan \theta}\right)$

$=\pi-2 \cos ^{-1}\left(\frac{\tan \left(1.2^{\circ}\right)}{\tan \bar{e}}\right)$

$2.0^{\circ}<\theta \leq \theta_{\max }$

$$
2\left[\sin ^{-1}\left(\frac{\sin \left(2^{\circ}\right)}{\sin \theta}\right)-\cos ^{-1}\left(\frac{\tan \left(1.2^{\circ}\right)}{\tan \theta}\right)\right]
$$

$\theta>\theta_{\max }$ 0

Table B - Range of $\phi$ for $\theta_{S}=1.2^{\circ}$.

Range of $\theta$

$$
\phi_{2}-\phi_{1}
$$

$0 \leq \theta \leq 2.0^{\circ}$

$\pi / 2$

$2.0^{\circ}<\theta \leq 2.4^{\circ}$
$2.4^{\circ}<\theta \leq \theta_{\max }$ $\sin ^{-1}\left(\frac{\sin \left(2^{\circ}\right)}{\sin \theta}\right)$ $\sin ^{-1}\left(\frac{\sin \left(2^{\circ}\right)}{\sin \theta}\right)-\cos ^{-1}\left(\frac{\tan \left(2.4^{\circ}\right)}{\tan \theta}\right)$ 
Table $C$ - Range of $\phi$ for $\theta_{S}=2^{\circ}$.

\begin{tabular}{ll}
\hline Range of $\theta$ & $\phi_{2}-\phi_{1}$ \\
\hline $0.8^{\circ} \leq \theta \leq \theta^{\prime}$ & $\cos ^{-1}\left(\frac{\tan \left(0.8^{\circ}\right)}{\tan \theta}\right)$ \\
$\theta^{\prime}<\theta \leq 3.2^{\circ}$ & $\sin ^{-1}\left(\frac{\sin \left(2^{\circ}\right)}{\sin \theta}\right)$ \\
$3.2^{\circ}<\theta \leq \theta_{\max }$ & $\sin ^{-1}\left(\frac{\sin \left(2^{\circ}\right)}{\sin \theta}\right)-\cos ^{-1}\left(\frac{\tan \left(3.2^{\circ}\right)}{\tan \theta}\right)$ \\
\hline
\end{tabular}

Table D - Range of $\phi$ for $\theta_{S}=4^{\circ}$.

Range of $\theta \quad \phi_{2}-\phi_{1}$

$\begin{array}{ll}2.8^{\circ} \leq \theta \leq \theta^{\prime} & \cos ^{-1}\left(\frac{\tan \left(2.8^{\circ}\right)}{\tan \theta}\right) \\ \theta^{\prime}<\theta \leq 5.2^{\circ} & \sin ^{-1}\left(\frac{\sin \left(2^{\circ}\right)}{\sin \theta}\right) \\ 5.2^{\circ}<\theta \leq \theta_{\max } & \sin ^{-1}\left(\frac{\sin \left(2^{\circ}\right)}{\sin \theta}\right)-\cos ^{-1}\left(\frac{\tan \left(5.2^{\circ}\right)}{\tan \theta}\right)\end{array}$

$$
I_{1}=-\left.\left[\frac{x \cos ^{-1} x}{\left(x^{2}+A^{2}\right)^{1 / 2}}+\frac{1}{2} \sin ^{-1}\left(\frac{1+A^{2}-2\left(1-x^{2}\right)}{1+A^{2}}\right)\right]\right|_{x_{1}} ^{x_{2}}
$$


where the limits of evaluation of the expression in the brackets are given by

$$
x_{1}=\frac{A}{\tan \theta_{1}} \quad, \quad x_{2}=\frac{A}{\tan \theta_{2}}
$$

The second type of integral is

$$
I_{2}=\int_{\theta_{1}}^{\theta_{2}} d \theta \sin \theta \sin ^{-1}\left(\frac{A}{\sin \theta}\right)
$$

where $A=\sin 2^{\circ} . I_{2}$ can be shown to be

$$
\begin{aligned}
I_{2}= & {\left[\frac{\left(A^{2}-x^{2}\right) \sin ^{-1} x}{x\left(x^{2}-A^{2}\right)^{1 / 2}}+\frac{1}{2} \sin ^{-1}\left(\frac{1-A^{2}-2\left(1-x^{2}\right)}{1-A^{2}}\right)\right.} \\
& \left.-\frac{A}{2} \sin ^{-1}\left(\frac{\left(A^{2}+1\right) x^{2}-2 A^{2}}{x^{2}\left(A^{4}-2 A^{2}+1\right)^{1 / 2}}\right)\right]\left.\right|_{x_{1}} ^{x_{2}}
\end{aligned}
$$

with $x_{1}$ and $x_{2}$ given by

$$
x_{1}=\frac{A}{\sin \theta_{1}} \quad, \quad x_{2}=\frac{A}{\sin \theta_{2}}
$$

Figures $G, K, I$, and $J$ show the solid angle as a function of $\theta$ for the four cases of figures $C, D, E$, and $F$. The bin size of $\theta$ is $1 \mathrm{mr}$, ie. $\theta_{2}-\theta_{1}=1 \mathrm{mr}$. The effects of the horizontal and vertical acceptance of the spectrometer show up quite clearly in the figures 


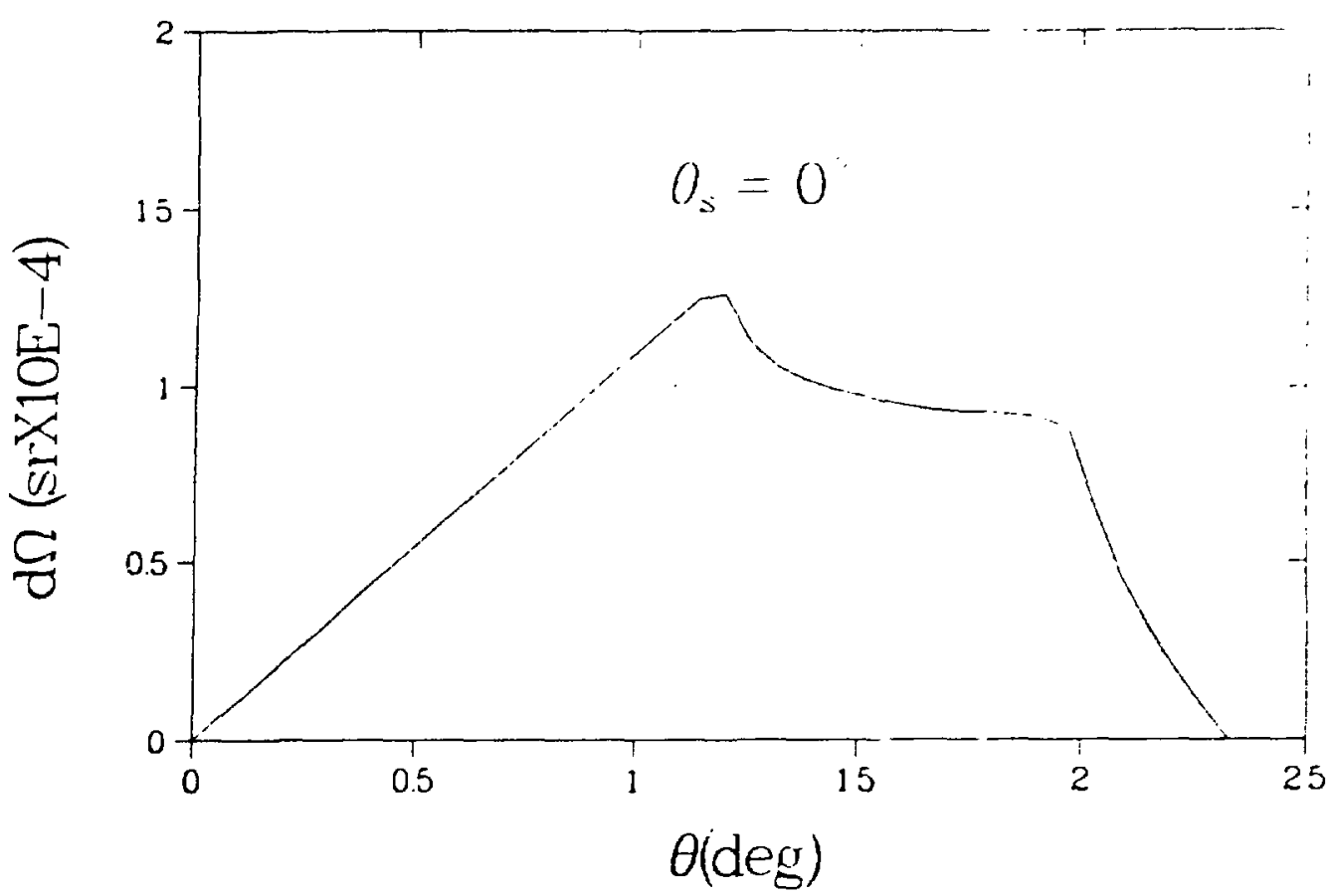

Fig. G

Solid angle dependence on $\theta$ for a $1 \mathrm{mr}$ bin size at $\theta_{S}=0^{\circ}$.

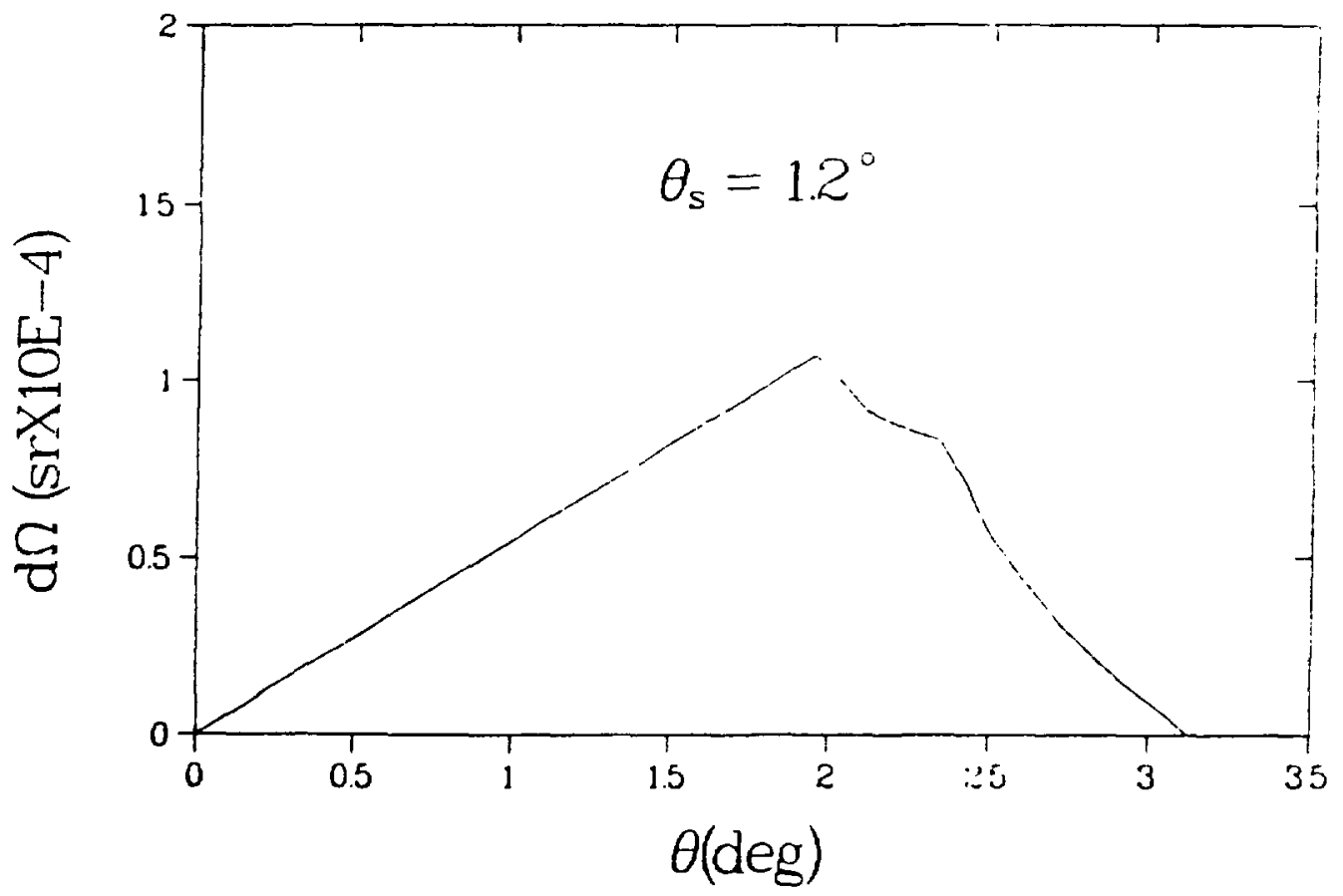

Fig. H

Solid angle dependence on $\theta$ for a $1 \mathrm{mr}$ bin size at $\theta_{S}=1.2^{\circ}$. 


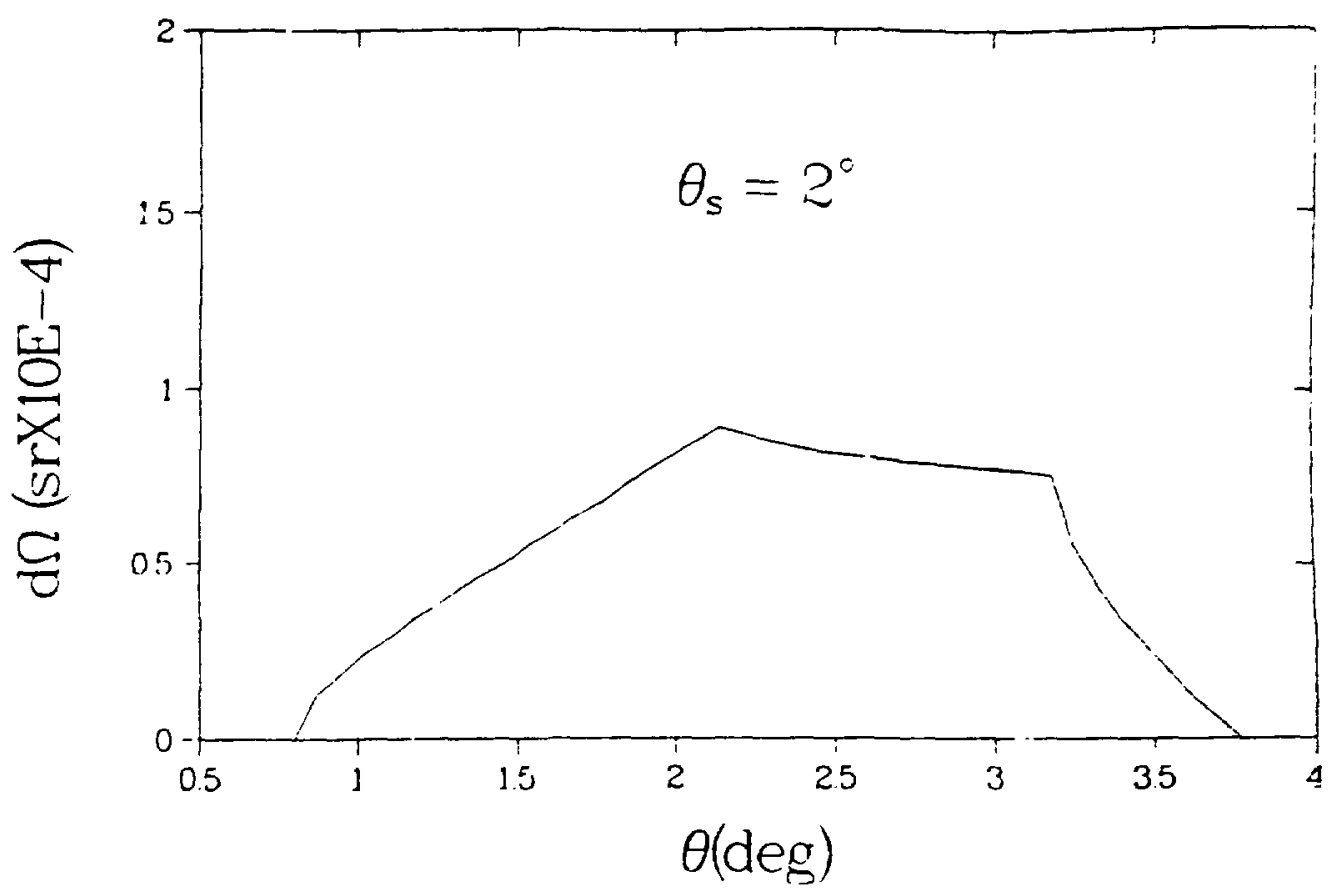

Fig. I

Solid angle dependence on $\theta$ for a $1 \mathrm{mr}$ bin size at $\theta_{S}=2^{\circ}$.

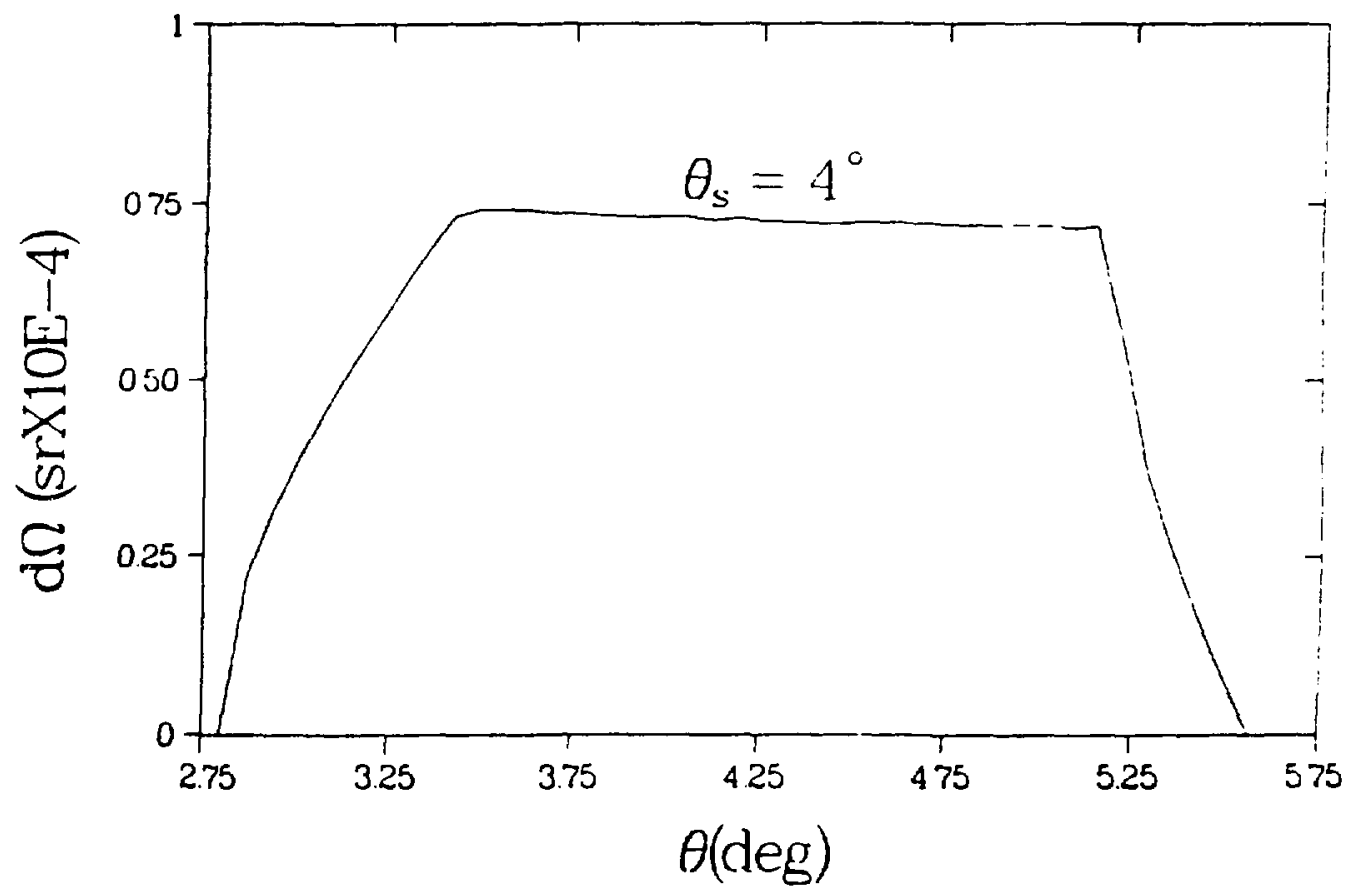

Fig. J

Solid angle dependence on $\theta$ for a $1 \mathrm{mr}$ bin size at $\theta_{S}=4^{\circ}$. 
where a marked change in behaviour can be seen at $\theta=1.2^{\circ}$ and $\theta$. $2^{\circ}$. 
Appendix B :

Multiple scattering

To calculate the contribution of multiple scattering to the measured difforential cross section, we use the multiple scattering theory of Nigam, Sundaresan, and Vu (NSW) ${ }^{1}$. The NSW theory is more accurate than the Moliere theory, although the Moliere theory does not give results which differ significantly from the NSU theory. For our particular case, an approximation to the NSW theory is adequate and easier to use. Ve shall therefore use the approximate NSW theory to obtain angular distribution curves for multiple scattering on the ${ }^{12} \mathrm{C},{ }^{40} \mathrm{Ca}$, and ${ }^{208} \mathrm{~Pb}$ targets.

In the NSW theory, the angle $x_{c}$ at which, on average, there is only one collision with $\theta>x_{c}$ is given by the expression

$$
x_{C}^{2}=0.1569\left(Z(Z+1) z^{2} / A\right)\left(t /(P B C)^{2}\right)
$$

where $Z$ is the atomic number of the target material, $A$ is its atomic veight, $z$ is the atomic number of the incident particle, $t$ is the target thickness in $\mathrm{g} / \mathrm{cm}^{2}$, and $\mathrm{PBC}$ is the momentum $\times$ velocity of the incident particle in MeV. Eqn.(1) is the same for the Moliere theory. The number of collisions that the incident particle

TMarion, J., and B. Zimmerman, Nuci. Inst. Meth. 5 i (1957) 93 
experiences while passing through the target is approximately $e^{b}$, where

$$
b=\ln \left(\frac{2730(Z+1) z^{1 / 3} z^{2} t}{A B^{2}}\right)-0.1544
$$

The angular distribution of the approximate NSW theory can then be calculated once $X_{c}$ and $b$ are known for the particular situation. The approximate NSW angular distribution is

$$
F(x)=\frac{1}{x_{c}^{2} B} \sum_{n=0}^{\infty} F_{n} \frac{1}{n ! B^{n}}
$$

where

$$
x=\frac{\theta}{X_{c} B^{1 / 2}}
$$

and $B$ is the root of

$$
B-\ln B=b
$$

Summing over the first few terms of eqn.(3) is sufficient. These terms are approximations to the complete NSW theory and have the form

$$
F_{0}=2 \exp \left(-x^{2}\right)
$$




$$
\begin{gathered}
F_{1}=\frac{1}{4} \int_{0}^{\Gamma} u^{3} J_{0}(u x) \ln \left(u^{2} / 4\right) \exp \left(-u^{2} / 4\right) d u \\
F_{2}=\frac{1}{16} \int_{0}^{\Gamma} u^{5} J_{0}(u x)\left(\ln \left(u^{4} / 4\right)\right)^{2} \exp \left(-u^{2} / 4\right) d u
\end{gathered}
$$

where

$$
I=B^{1 / 2} \exp ((B-1.5) / 2.2)
$$

In order to use the approximate NSW form for the angular distribution, first $X_{c}$ and $b$ are calculated and $B$ found from $b$ through eqn.(5). However, the validity of the approximate NSW angular distribution is contingent on $B$ falling within an allowable range. The limits of $B$ come from two basic considerations. First, since the theory is a statistical one, the number of collisions experienced by the incident particle should not be less than 10-20. This means that $B$ must be greater than about 4 for the theory to be valid. Secondly, the scattered particle must not lose more than - 20\% of its initial energy in traversing the target. This means that $t$ should not be too large and $B$ should therefore be less than about 15. These criteria are easily satisfied in this experiment and so the approximate NSW multiple scattering theory is appropriate.

Figure $K$ shows the multiple scattering angular distributions calculated from the approximate NSW theory for the ${ }^{40} \mathrm{Ca}$ target. The dashed line in the figure is a Gaussian distribution with the width chosen to be the value of $x$ for which $F(x)$ has its 1/e value. A Gaussian distribution is often used to get a rough estimate of the 


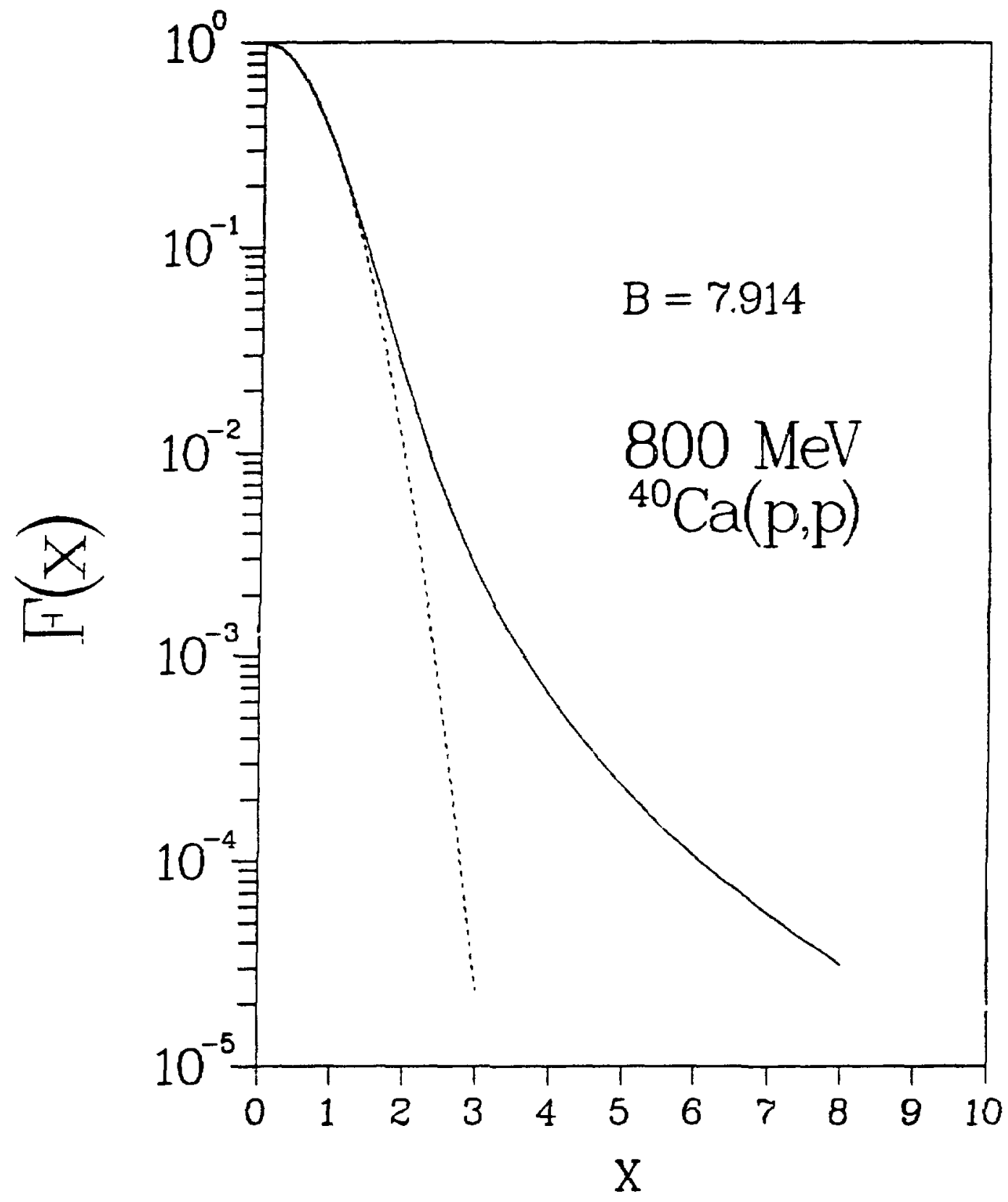

Fig. K

Multiple scattering distribution for $800, \mathrm{HeV}$ protons incident of a ${ }^{\circ} \mathrm{Ca}$ target $73.6 \mathrm{mg} / \mathrm{cm}^{2}$ thick. 
multiple scattering distribution. However, as can be seen from figure $k$, the Gaussian distribution underestimates the multiple scattering contributions since more particles are scattered in the forward direction by it than by the distribution generated from the approximate NSW theory.

The value of $B$ for the case of $800 \mathrm{MeV}$ protons on ${ }^{40} \mathrm{Ca}$ target $73.6 \mathrm{mg} / \mathrm{cm}^{2}$ thick is 7.9139. For the ${ }^{12} \mathrm{C}$ and ${ }^{208} \mathrm{~Pb}$ targets, $B$ has the values 8.3023 and 8.4923 respectively. Since the $B$ values for these targets are about the same, the plots of $F(x)$ vs. $x$ for each target look almost identical. To see a difference, one has to plot the distribution as a function of $\theta$. This is done for each target in figure $L$. 


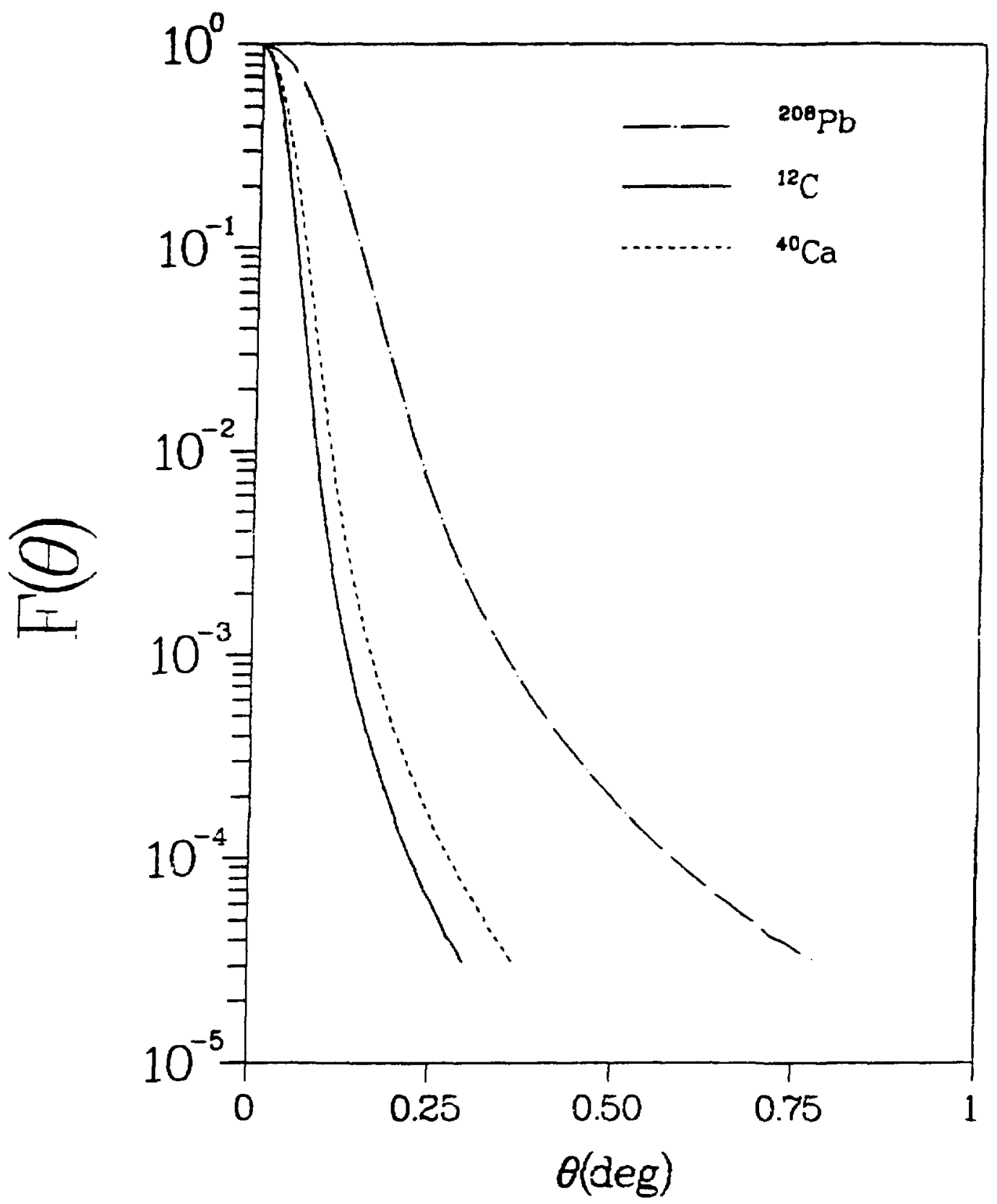

Fig. L

Multiple scattering distributions for the $22 \mathrm{C},{ }^{\circ} \mathrm{Ca}$, and $20 . \mathrm{Pb}$ targets. 
Appendix C:

Statistical considerations of beam intensity monftoring

Under the condition of a pulsed bunched beam, statistical theory can be applied to get a good measure of the bean intensity. The fact that the beam is entirely contained in micropulses about 0.25 ns wide 100 ns apart allows the statistical nature of the beam to be studied. To do this, ve place two scintillators $s_{1}$ and $s_{2}$ in the beam and form a coincidence between the two:

$$
N_{p}=s_{1} \cdot s_{2}
$$

He also form a coincidence between this coincidence and itself delayed by the separation between micropulses:

$$
N_{d}=\left(s_{1} \cdot s_{2}\right) \cdot\left(s_{1} \cdot s_{2}\right)_{100}
$$

$N_{p}$ therefore increments the scaler whenever there are one or more particles in a micropulse and $N_{d}$ increments when there are one or more particles in each of two adjacent micropulses. Scaling these two quantities will yield the number of beam particles if the distribution of beam particles in a nicropulse follows Poisson statistics.

Assuning Polsson distribution of particles in a micropulse, the probability of $n$ particles in one micropulse is 


$$
p_{n}=\frac{x^{n} e^{-x}}{n !}
$$

where $x$ is the average number of particies in a mcropulse. $x$ can be expreased as the ratlo of the total number of beam particles $N_{b}$ to the total number of atcropulses $N_{m}$ :

$$
x=\frac{N_{b}}{N_{m}}
$$

SInce the sum of eqn.(3) over all $n$ must be equal to one, the probability that there is one or more particles in a micropulse, $n \geq 1$, Is just

$$
\sum_{n=1}^{\infty} P_{n}=1-P_{0}=1-e^{-x}
$$

The probabllity that there are one or more particles in sach of two adjacent alcropulses is simply

$$
\left(\sum_{n=1}^{\infty} P_{n}\right)\left(\sum_{n=1}^{\infty} P_{n}\right)=\left(1-e^{-x}\right)^{2}
$$

$N_{p}$ is a measurement of eqn. (5) and $N_{d}$ is a measurement of eqn.(6).

$$
\begin{aligned}
& N_{p}=N_{m}\left(1-e^{-x}\right) \\
& N_{d}=N_{m}\left(1-e^{-x}\right)^{2}
\end{aligned}
$$

The idea is to express $N_{b}$ in terms of the two measurable quantities 
$N_{p}$ and $N_{d}$. Eqns. (4), (7) and (8) can be combined to do this. Taking the ratio of eqn. (8) to eqn. (7) ve have

$$
\frac{N_{d}}{N_{p}}=1-e^{-x}
$$

This can be solved for $x$, giving

$$
x=-\ln \left(1-\frac{N_{d}}{N_{p}}\right)
$$

Similarly, by taking the ratio of the square of eqn. (7) to eqn. (8) we have

$$
N_{m}=\frac{N_{p}^{2}}{N_{d}}
$$

Combining eqns.(4), (10) and (11) ylelds the desired result:

$$
N_{b}=-\frac{N_{p}^{2}}{N_{d}} \ln \left(1-\frac{N_{d}}{N_{p}}\right)
$$

As vay of checking the number of beam particles counted using eqn. (12), ve can form another coincidence $N_{d 2}$

$$
N_{d 2}=\left(s_{1} \cdot s_{2}\right) \cdot\left(s_{1} \cdot s_{2}\right)_{100} \cdot\left(s_{1} \cdot s_{2}\right)_{200}
$$


$N_{d 2}$ w1ll therefore be measurement of the probability that there are one or more particles in each of three adjacent micropulses.

$$
N_{d 2}=N_{m}\left(1-e^{-x}\right)^{3}
$$

Proceeding as before using eqns. (7) and (14) leads to the result

$$
N_{b}=-\left(\frac{N_{p}^{3}}{N_{d 2}}\right)^{1 / 2} \ln \left(1-\left(\frac{N_{d 2}}{N_{p}}\right)^{1 / 2}\right)
$$

It is clear that this process can be repeated with $n$ 100ns delays. That is, one can form the coincidence

$$
N_{d n}=\left(s_{1} \cdot s_{2}\right) \cdot\left(s_{1} \cdot s_{2}\right)_{100} \cdots\left(s_{1} \cdot s_{2}\right)_{100 \times n}
$$

which is a aeasurement of the probability that there are one or more particles in each of $n$ adjacent micropulses. And the number of beam particles counted will be

$$
N_{b}=-\left(\frac{N_{p}^{n+1}}{N_{d n}}\right)^{1 / n} \ln \left(1-\left(\frac{N_{d n}}{N_{p}}\right)^{1 / n}\right)
$$


Append Ix $D$ :

Tabulation of data

Table E - Relative differential cross sections for ${ }^{208} \mathrm{~Pb}(\mathrm{p}, \mathrm{p})$ at $800 \mathrm{MeV}$.

$\theta_{c m}($ Deg $) \quad q(\mathrm{MeV} / \mathrm{c}) \quad \mathrm{d \sigma} / d g(\mathrm{rel}$. unlts)

\begin{tabular}{|c|c|c|c|}
\hline 0.98 & 24.80 & 355. & \pm 7.6 \\
\hline 1.15 & 29.19 & 167. & \pm 3.8 \\
\hline 1.33 & 33.59 & 99.6 & \pm 2.4 \\
\hline 1.50 & 37.97 & 62.0 & \pm 1.7 \\
\hline 1.67 & 42.36 & 43.7 & \pm 1.3 \\
\hline 1.84 & 46.75 & 31.6 & \pm 1.0 \\
\hline 2.02 & 51.14 & 24.5 & \pm .86 \\
\hline
\end{tabular}

1.51

1.69

1.86

2.03

2.21

2.38

2.55

2.73

2.90

3.07

3.25

3.42
38.29

42.68

47.07

51.46

55.85

60.24

64.63

69.01

73.40

77.79

82.18

86.57
232. \pm 2.8

162. \pm 2.1

121. \pm 1.6

$91.6 \pm 1.3$

$70.9 \pm 1.1$

$55.0 \pm .90$

$42.0 \pm .75$

$32.5 \pm .63$

$23.3 \pm .51$

$17.0 \pm .42$

$12.1 \pm .35$

$8.07 \pm .28$ 


$\begin{array}{lrl}3.55 & 89.76 & 7.24 \pm .19 \\ 3.72 & 94.14 & 5.30 \pm .16 \\ 3.89 & 98.53 & 3.41 \pm .13 \\ 4.07 & 102.92 & 2.57 \pm .11 \\ 4.24 & 107.30 & 1.99 \pm .10 \\ 4.41 & 111.69 & 1.84 \pm .10 \\ 4.59 & 116.08 & 1.92 \pm .10 \\ 4.76 & 120.46 & 1.96 \pm .10 \\ 4.93 & 124.85 & 2.12 \pm .10\end{array}$

Table $F$ - Relative differential cross sections for ${ }^{40} \mathrm{Ca}(\mathrm{p}, \mathrm{p})$ at $800 \mathrm{MeV}$.

$\theta_{\mathrm{cm}}(\mathrm{Deg}) \quad \mathrm{q}(\mathrm{MeV} / \mathrm{c}) \quad \mathrm{do} / \mathrm{dg}(\mathrm{rel}$. units)

$\begin{array}{ll}1.57 & 38.29 \\ 1.75 & 42.68 \\ 1.93 & 47.07 \\ 2.11 & 51.46 \\ 2.29 & 55.85 \\ 2.47 & 60.24 \\ 2.65 & 64.62\end{array}$

$83.7 \pm .83$
$45.4 \pm .56$
$29.1 \pm .43$
$20.5 \pm .36$
$15.9 \pm .31$
$12.6 \pm .27$
$10.5 \pm .25$

1.01

24.80

29.19

1.38

33.59

1.56

37.97

1.74

42.36

1.91

46.75

2.09

51.14

$89.8 \pm 1.1$

$67.6 \pm .85$

$55.8 \pm .74$

$45.6 \pm .64$

$41.9 \pm .61$

$37.5 \pm .57$

$33.4 \pm .52$ 


$\begin{array}{llll}2.83 & 69.01 & 29.7 & \pm .49 \\ 3.01 & 73.40 & 25.6 & \pm .44 \\ 3.19 & 77.79 & 24.1 & \pm .43 \\ 3.37 & 82.18 & 21.9 \pm .40 \\ 3.55 & 86.56 & 18.9 \pm .37\end{array}$

3.50
3.68
3.86
4.04
4.22
4.40
4.58
4.76
4.93
5.11

85.37

89.75

94.14

98.53

102.91

107.30

111.69

116.07

120.46

124.84
$18.8 \pm .48$

$19.1 \pm .48$

$18.6 \pm .48$

$16.2 \pm .44$

$14.5 \pm .41$

$11.0 \pm .35$

$10.7 \pm .35$

$9.18 \pm .32$

$7.69 \pm .29$

$5.97 \pm .25$

Table G - Relative differential cross sections for ${ }^{12} \mathrm{C}(\mathrm{p}, \mathrm{p})$ at $800 \mathrm{MeV}$.

$\begin{array}{lrr}1.11 & 24.80 & 55.0 \pm .69 \\ 1.31 & 29.19 & 32.5 \pm .49 \\ 1.51 & 33.58 & 22.6 \pm .40 \\ 1.71 & 37.97 & 16.6 \pm .33 \\ 1.90 & 42.36 & 13.7 \pm .30 \\ 2.10 & 46.75 & 11.3 \pm .27 \\ 2.30 & 51.14 & 9.58 \pm .25\end{array}$




$\begin{array}{llll}1.72 & 38.29 & 69.5 & \pm .93 \\ 1.92 & 42.68 & 56.2 \pm .79 \\ 2.12 & 47.07 & 49.3 \pm .72 \\ 2.31 & 51.46 & 42.5 \pm .65 \\ 2.51 & 55.84 & 39.6 \pm .62 \\ 2.71 & 60.23 & 36.2 \pm .58 \\ 2.91 & 64.62 & 34.8 \pm .57 \\ 3.10 & 69.01 & 31.9 \pm .54 \\ 3.30 & 73.40 & 29.9 \pm .51 \\ 3.50 & 77.78 & 28.5 \pm .50 \\ 3.69 & 82.17 & 27.3 \pm .49 \\ 3.89 & 86.56 & 25.1 \pm .46\end{array}$

\begin{tabular}{|c|c|c|c|c|}
\hline 4.04 & 89.74 & 24.5 & \pm & .73 \\
\hline 4.23 & 94.13 & 24.7 & \pm & .74 \\
\hline 4.43 & 98.52 & 23.0 & \pm & .70 \\
\hline 4.63 & 102.90 & 22.5 & \pm & .70 \\
\hline 4.82 & 107.28 & 20.3 & \pm & .64 \\
\hline 5.02 & 111.67 & 19.3 & \pm & .64 \\
\hline 5.22 & 116.05 & 18.0 & \pm & .61 \\
\hline 5.42 & 120.44 & 15.8 & \pm & .56 \\
\hline 5.61 & 124.82 & 16.0 & \pm & .56 \\
\hline
\end{tabular}

Table $\mathrm{H}$ - Absolute differential cross sections for ${ }^{208} \mathrm{~Pb}(\mathrm{p}, \mathrm{p})$ at $800 \mathrm{MeV}$.

$\theta_{\mathrm{cm}}(\mathrm{Deg}) \quad q(\mathrm{MeV} / \mathrm{c}) \quad \mathrm{d \sigma} / \mathrm{d} Q(\mathrm{mb} / \mathrm{sr})$

$\begin{array}{lll}0.98 & 24.80 & 3120000 . \pm 72000 . \\ 1.15 & 29.19 & 1470000 . \pm 36000 .\end{array}$




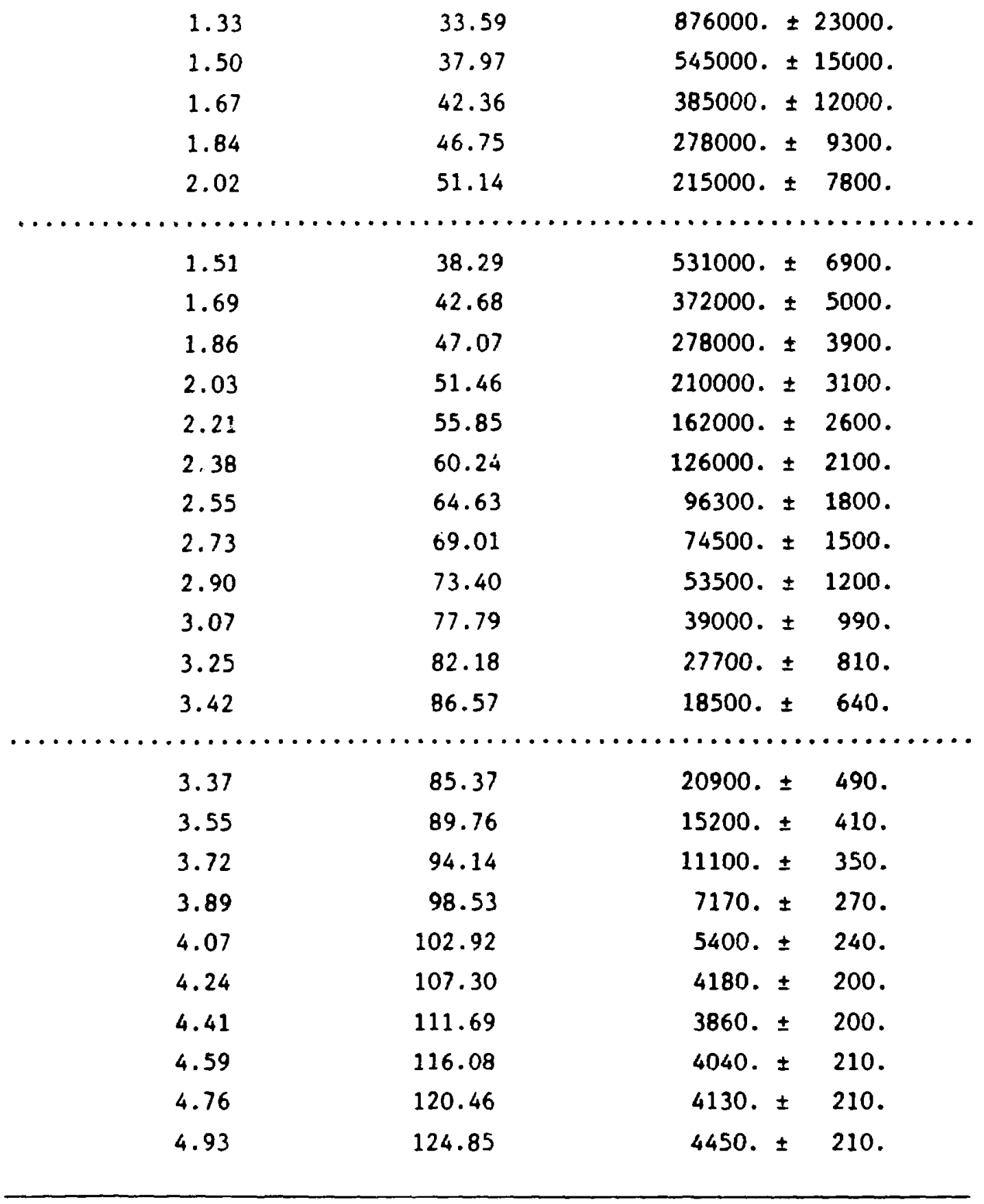


Table I - Absolute differential cross sections for ${ }^{40} \mathrm{Ca}(p, p)$ at $800 \mathrm{MeV}$.

$\theta_{\mathrm{cm}}(\mathrm{Deg}) \quad \mathrm{q}(\mathrm{MeV} / \mathrm{c}) \quad \mathrm{do} / \mathrm{d} Q(\mathrm{mb} / \mathrm{sr})$

$\begin{array}{llr}1.01 & 24.80 & 221000 . \pm 3700 . \\ 1.20 & 29.19 & 120000 . \pm 2200 . \\ 1.38 & 33.59 & 76700 . \pm 1500 . \\ 1.56 & 37.97 & 54000 . \pm 1200 . \\ 1.74 & 42.36 & 41900 . \pm 990 . \\ 1.91 & 46.75 & 33200 . \pm 840 . \\ 2.09 & 51.14 & 27800 . \pm 750 .\end{array}$

$\begin{array}{lll}1.57 & 38.29 & 54100 . \pm 870 . \\ 1.75 & 42.68 & 40700 . \pm 680 . \\ 1.93 & 47.07 & 33600 . \pm 580 . \\ 2.11 & 51.46 & 27500 . \pm 490 . \\ 2.29 & 55.85 & 25200 . \pm 460 . \\ 2.47 & 60.24 & 22600 . \pm 420 . \\ 2.65 & 64.62 & 20100 . \pm 380 . \\ 2.83 & 69.01 & 17900 . \pm 350 . \\ 3.01 & 73.40 & 15400 . \pm 320 . \\ 3.19 & 77.79 & 14500 . \pm 300 . \\ 3.37 & 82.18 & 13200 . \pm 280 . \\ 3.55 & 86.56 & 11400 . \pm 250 .\end{array}$

$\begin{array}{lr}3.50 & 85.37 \\ 3.68 & 89.75 \\ 3.86 & 94.14 \\ 4.04 & 98.53 \\ 4.22 & 102.91 \\ 4.40 & 107.30\end{array}$

10400. \pm 290 .

10500. \pm 300 .

10300. \pm 290 .

8960. \pm 270 .

8020. \pm 250 .

$6090 . \pm 210$. 


$\begin{array}{lll}4.58 & 111.69 & 5900 . \pm 210 . \\ 4.76 & 116.07 & 5060 . \pm 190 . \\ 4.93 & 120.46 & 4240 . \pm 170 . \\ 5.11 & 124.84 & 3290 . \pm 140 .\end{array}$

Table $J$ - Absolute differential cross sections for ${ }^{12} \mathrm{C}(p, p)$ at $800 \mathrm{MeV}$.

$\theta_{\mathrm{cm}}(\mathrm{Deg}) \quad \mathrm{q}(\mathrm{MeV} / \mathrm{c}) \quad \mathrm{do} / \mathrm{de}(\mathrm{mb} / \mathrm{sr})$

$\begin{array}{llr}1.11 & 24.80 & 22700 . \pm 420 . \\ 1.31 & 29.19 & 13400 . \pm 280 . \\ 1.51 & 33.58 & 9310 . \pm 210 . \\ 1.71 & 37.97 & 6820 . \pm 170 . \\ 1.90 & 42.36 & 5660 . \pm 150 . \\ 2.10 & 46.75 & 4650 . \pm 130 . \\ 2.30 & 51.14 & 3950 . \pm 120 .\end{array}$

1.72

1.92

2.12

2.31

2.51

2.71

2.91

3.10

3.30

3.50

3.69

3.89
38.29

42.68

47.07

51.46

55.84

60.23

64.62

69.01

73.40

77.78

82.17

86.56
6640. \pm 110 .

5370. \pm 96 .

4700. \pm 86 .

4060. \pm 76 .

$3780 . \pm 72$.

$3450 . \pm 67$.

$3330 . \pm 65$.

3040. \pm 61 .

2860. \pm 58 .

2720 . \pm 56 .

2610. \pm 55 .

2400. \pm 51 . 


$\begin{array}{lrl}4.04 & 89.74 & 2150 . \pm 70 . \\ 4.23 & 94.13 & 2160 . \pm 70 . \\ 4.43 & 98.52 & 2010 . \pm 67 . \\ 4.63 & 102.90 & 1970 . \pm 66 . \\ 4.82 & 107.28 & 1770 . \pm 61 . \\ 5.02 & 111.67 & 1690 . \pm 60 . \\ 5.22 & 116.05 & 1580 . \pm 57 . \\ 5.42 & 120.44 & 1380 . \pm 52 . \\ 5.61 & 124.82 & 1400 . \pm 52 .\end{array}$

Table K - Analyzing powers for ${ }^{208} \mathrm{~Pb}(\mathrm{p}, \mathrm{p})$ at $800 \mathrm{MeV}$.

$\theta_{\mathrm{cm}}(\mathrm{Deg}) \quad \mathrm{q}(\mathrm{HeV} / \mathrm{C}) \quad A_{y}$

$\begin{array}{llr}0.98 & 24.80 & 0.030 \pm 0.039 \\ 1.15 & 29.19 & 0.006 \pm 0.042 \\ 1.33 & 33.59 & 0.043 \pm 0.045 \\ 1.50 & 37.97 & -0.003 \pm 0.049 \\ 1.67 & 42.36 & 0.088 \pm 0.054 \\ 1.84 & 46.75 & 0.106 \pm 0.059 \\ 2.02 & 51.14 & 0.070 \pm 0.064\end{array}$

1.51

1.69

1.86

2.03

2.21

2.38
38.29

42.68

47.07

51.46

55.85

60.24
$0.014 \pm 0.015$

$0.048 \pm 0.015$

$0.051 \pm 0.016$

$0.082 \pm 0.017$

$0.092 \pm 0.018$

$0.080 \pm 0.020$ 


$\begin{array}{llll}2.55 & 64.63 & 0.103 & \pm 0.021 \\ 2.73 & 69.01 & 0.066 & \pm 0.023 \\ 2.90 & 73.40 & 0.105 & \pm 0.026 \\ 3.07 & 77.79 & 0.106 \pm 0.030 \\ 3.25 & 82.18 & 0.111 \pm 0.035 \\ 3.42 & 86.57 & 0.086 \pm 0.041\end{array}$

\begin{tabular}{|c|c|c|c|}
\hline 3.37 & 85.37 & 0.092 & \pm 0.026 \\
\hline 3.55 & 89.76 & 0.103 & \pm 0.031 \\
\hline 3.72 & 94.14 & 0.117 & \pm 0.035 \\
\hline 3.89 & 98.53 & -0.014 & \pm 0.045 \\
\hline 4.07 & 102.92 & 0.136 & \pm 0.050 \\
\hline 4.24 & 107.30 & 0.128 & \pm 0.056 \\
\hline 4.41 & 111.69 & 0.242 & \pm 0.057 \\
\hline 4.59 & 116.08 & 0.295 & \pm 0.055 \\
\hline 4.76 & 120.46 & 0.160 & \pm 0.057 \\
\hline 4.93 & 124.85 & 0.258 & \pm 0.052 \\
\hline
\end{tabular}

Table L - Analyzing powers for

${ }^{40} \mathrm{Ca}(\mathrm{p}, \mathrm{p})$ at $800 \mathrm{MeV}$.

$\theta_{\mathrm{cm}}(\mathrm{Deg}) \quad \mathrm{q}(\mathrm{MeV} / \mathrm{C}) \quad \mathrm{A}_{\mathrm{y}}$

$\begin{array}{llll}1.01 & 24.80 & 0.039 & \pm 0.018 \\ 1.20 & 29.19 & 0.013 \pm 0.022 \\ 1.38 & 33.59 & 0.029 \pm 0.026 \\ 1.56 & 37.97 & 0.005 & \pm 0.031 \\ 1.74 & 42.36 & 0.057 & \pm 0.034 \\ 1.91 & 46.75 & 0.074 \pm 0.038 \\ 2.09 & 51.14 & 0.074 \pm 0.042\end{array}$




$\begin{array}{lll}1.57 & 38.29 & 0.058 \pm 0.014 \\ 1.75 & 42.68 & 0.062 \pm 0.015 \\ 1.93 & 47.07 & 0.075 \pm 0.016 \\ 2.11 & 51.46 & 0.111 \pm 0.017 \\ 2.29 & 55.85 & 0.101 \pm 0.017 \\ 2.47 & 60.24 & 0.116 \pm 0.018 \\ 2.65 & 64.62 & 0.126 \pm 0.019 \\ 2.83 & 69.01 & 0.176 \pm 0.019 \\ 3.01 & 73.40 & 0.153 \pm 0.020 \\ 3.19 & 77.79 & 0.154 \pm 0.021 \\ 3.37 & 82.18 & 0.140 \pm 0.022 \\ 3.55 & 86.56 & 0.173 \pm 0.023\end{array}$

3.50

85.37

$0.149 \pm 0.030$

3.68

89.75

$0.196 \pm 0.030$

3.86

94.14

$0.171 \pm 0.030$

4.04

98.53

$0.214 \pm 0.032$

4.22

102.91

$0.210 \pm 0.034$

4.40

107.30

$0.197 \pm 0.038$

4.58

111.69

$0.202 \pm 0.039$

4.76

116.07

$0.203 \pm 0.041$

4.93

120.46

$0.238 \pm 0.045$

5.11

124.84

$0.244 \pm 0.049$ 
Table M - Analyz:ng powers for

${ }^{12} \mathrm{C}(\mathrm{p}, \mathrm{p})$ at $800 \mathrm{MeV}$.

\begin{tabular}{|c|c|c|c|}
\hline$\theta_{\mathrm{cm}}($ Deg $)$ & $q(\mathrm{MeV} / \mathrm{c})$ & A & $y$ \\
\hline 1.11 & 24.80 & 0.079 & \pm 0.027 \\
\hline 1.31 & 29.19 & 0.076 & \pm 0.033 \\
\hline 1.51 & 33.58 & 0.024 & \pm 0.039 \\
\hline 1.71 & 37.97 & 0.011 & \pm 0.044 \\
\hline 1.90 & 42.36 & 0.073 & \pm 0.048 \\
\hline 2.10 & 46.75 & 0.095 & \pm 0.052 \\
\hline 2.30 & 51.14 & 0.147 & \pm 0.056 \\
\hline , . . . . & $\cdots \cdots \cdot$ & . . . & $\cdots \cdots \cdots$ \\
\hline 1.72 & 38.29 & 0.080 & \pm 0.016 \\
\hline 1.92 & 42.68 & 0.066 & \pm 0.017 \\
\hline 2.12 & 47.07 & 0.099 & \pm 0.017 \\
\hline 2.31 & 51.46 & 0.111 & \pm 0.018 \\
\hline 2.51 & 55.84 & 0.104 & \pm 0.018 \\
\hline 2.71 & 60.23 & 0.102 & \pm 0.019 \\
\hline 2.91 & 64.62 & 0.115 & \pm 0.019 \\
\hline 3.10 & 69.01 & 0.135 & \pm 0.020 \\
\hline 3.30 & 73.40 & 0.140 & \pm 0.020 \\
\hline 3.50 & 77.78 & 0.213 & \pm 0.020 \\
\hline 3.69 & 82.17 & 0.159 & \pm 0.021 \\
\hline 3.89 & 86.56 & 0.160 & \pm 0.021 \\
\hline 4.04 & 89.74 & 0.154 & \pm 0.036 \\
\hline 4.23 & 94.13 & 0.144 & \pm 0.036 \\
\hline 4.43 & 98.52 & 0.212 & \pm 0.036 \\
\hline 4.63 & 102.90 & 0.145 & \pm 0.037 \\
\hline 4.82 & 107.28 & 0.207 & \pm 0.038 \\
\hline 5.02 & 111.67 & 0.183 & \pm 0.039 \\
\hline
\end{tabular}




$\begin{array}{llll}5.22 & 116.05 & 0.201 & \pm 0.040 \\ 5.42 & 120.44 & 0.262 & \pm 0.042 \\ 5.61 & 124.82 & 0.190 & \pm 0.042\end{array}$

